\title{
3-2-1 foliations for Reeb flows on $S^{3}$
}

\author{
Carolina Lemos de Oliveira
}

TESE APRESENTADA AO

Instituto DE Matemática e Estatística

DA Universidade DE SÃo PaUlo

PARA OBTENÇÃO DO TÍTULO

DE Doutor EM CiÊnCIAS

\author{
Programa de Pós-Graduação em Matemática \\ Orientador: Prof. Dr. Pedro Antonio Santoro Salomão
}

Este trabalho contou com o apoio financeiro da FAPESP e da CAPES

São Paulo, março de 2020 



\title{
3-2-1 foliations for Reeb flows on $S^{3}$
}

\author{
Carolina Lemos de Oliveira
}

Esta versão da tese contém as correções e alterações sugeridas pela comissão julgadora durante a defesa da versão original do trabalho, realizada em 6/3/2020. Uma cópia da versão original está disponível no Instituto de Matemática e Estatística da Universidade de São Paulo.

Comissão julgadora:

- Prof. Dr. Pedro Antonio Santoro Salomão (orientador)

- Prof. Dr. André Vanderlinde da Silva

- $\operatorname{Prof}^{\mathrm{a}}$. Dr ${ }^{\mathrm{a}}$. Naiara Vergian de Paulo

- Prof. Dr. Umberto Leone Hryniewicz

- Prof. Dr. Alexsandro Schneider 



\section{Agradecimentos}

Primeiramente, agradeço ao meu orientador, Pedro Salomão, por propor este projeto e pelo suporte ao longo de todas as etapas da realização do mesmo. Este projeto só foi possível graças às suas ideias, esclarecimento de dúvidas, sugestões e correções.

Agradeço aos membros da banca, Umberto Hryniewicz, Naiara de Paulo, Alexsandro Schneider e André Vanderlinde, não só pelas correções e sugestões para a tese, mas também por todas as discussões construtivas e pelo incentivo ao longo da realização deste projeto.

Também agradeço a Ana Kelly de Oliveira e Keon Choi por todas as discussões e sugestões que ajudaram direta ou indiretamente nesta tese.

Por último, agradeço à CAPES e à FAPESP pelo apoio financeiro. 


\section{Resumo}

\section{Folheações do tipo 3-2-1 para fluxos de Reeb em $S^{3}$}

Neste trabalho, estudamos sistemas globais de seções transversais para fluxos de Reeb associados a formas de contato tight na 3-esfera. Tais fluxos incluem, em particular, fluxos Hamiltonianos em $\mathbb{R}^{4}$ restritos a níveis de energia regulares estrelados. Um sistema global de seções transversais adaptado a um fluxo em $S^{3}$ é uma folheação singular de $S^{3}$ cujo conjunto singular, chamado de amarração, consiste de um número finito de órbitas periódicas e as folhas regulares são transversais ao fluxo. Como demonstrado por $\mathrm{H}$. Hofer, K. Wysocki e E. Zehnder em [HWZ03], fluxos de Reeb associados a formas de contato tight não degeneradas em $S^{3}$ admitem um sistema global de seções transversais, cujas folhas regulares são esferas furadas. Tais sistemas são construídos como a projeção em $S^{3}$ de uma folheação de $\mathbb{R} \times S^{3}$ por curvas pseudo-holomorfas.

Utilizando a teoria de curvas pseudo-holomorfas em simplectizações, estudamos a existência de um tipo de sistema de seções transversais, que chamamos de folheação 3-2-1, possuindo exatamente três órbitas na amarração, com índices de Conley-Zehnder respectivamente 3, 2 e 1 . Mais precisamente, apresentamos condições suficientes sob as quais três órbitas de Reeb formam a amarração de uma folheação 3-2-1.

Palavras-chave: Dinâmica Hamiltoniana, Fluxos de Reeb, Curvas pseudoholomorfas, Folheações de energia finita

Este trabalho foi financiado pelo processo nº 2016/10466-5, Fundação de Amparo à Pesquisa do Estado de São Paulo (FAPESP). 
O presente trabalho foi realizado com apoio da Coordenação de Aperfeiçoamento de Pessoal de Nível Superior - Brasil (CAPES) - Código de Financiamento 001. 


\section{Abstract}

In this work, we study global systems of transversal sections for Reeb flows associated with tight contact forms on the 3-sphere. These flows include, in particular, Hamiltonian flows on $\mathbb{R}^{4}$ restricted to star-shaped regular energy levels. A global system of transversal sections naturally generalizes the concept of global surface of section. It is a singular foliation of $S^{3}$ whose singular set consists of finitely many periodic orbits, called binding orbits, and the regular leaves are transverse to the flow. As proved by H. Hofer, K. Wysocki and E. Zehnder in [HWZ03, Reeb flows associated with non-degenerate tight contact forms on $S^{3}$ admit a global system of transversal sections whose regular leaves are punctured spheres. Such system is the projection to $S^{3}$ of a foliation of $\mathbb{R} \times S^{3}$ by pseudo-holomorphic curves.

The aim of this work is to use the theory of pseudo-holomorphic curves in symplectizations to study the existence of a particular type of system of transversal sections, called 3-2-1 foliation, which has exactly three binding orbits with Conley-Zehnder indices respectively 3, 2 and 1. More precisely, we give sufficient conditions under which three Reeb orbits are the binding orbits of a 3-2-1 foliation.

Keywords: Reeb Flows, Hamiltonian dynamics, finite energy foliations, pseudoholomorphic curves

This study was financed by grant \#2016/10466-5, São Paulo Reserch Fundation (FAPESP).

This study was financed in part by the Coordenação de Aperfeiçoamento de Pessoal de Nível Superior - Brasil (CAPES) - Finance Code 001. 


\section{Contents}

1 Contact geometry and Reeb dynamics 5

1.1 Contact structures . . . . . . . . . . . . . . . 5

1.1.1 Hypersurfaces of contact type . . . . . . . . 6

1.1.2 Symplectization of a contact manifold . . . . . . . . 7

1.1.3 Hamiltonian dynamics . . . . . . . . . . . . 8

1.1.4 Classification of contact structures in dimension 3 . . . 8

1.2 Reeb orbits . . . . . . . . . . . . . . . . . . . 9

1.2.1 The Conley-Zehnder index . . . . . . . . . . . . 11

1.3 The asymptotic operator . . . . . . . . . . . . . . 15

1.4 The action functional . . . . . . . . . . . . . . . 20

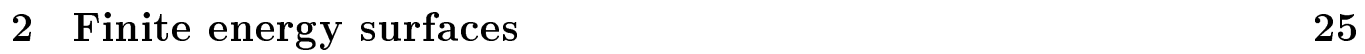

2.1 Almost complex structures in symplectizations . . . . . . . 25

2.2 Finite energy surfaces . . . . . . . . . . . . . . 26

$2.2 .1 \quad$ Asymptotic behavior . . . . . . . . . . . . 28

$2.2 .2 \quad$ Somewhere injective curves . . . . . . . . . . . 30

2.2 .3 Algebraic invariants . . . . . . . . . . . . . . 32

2.2 .4 Fredholm theory . . . . . . . . . . . 33

2.3 Bubbling-off analysis . . . . . . . . . . . . . . . 34

2.3.1 Elliptic Regularity and compactness . . . . . . . . . . 34

2.3 .2 Bubbling . . . . . . . . . . . . . 35

2.3 .3 Germinating sequences . . . . . . . . . . 37

$2.3 .4 \quad$ Soft-rescaling near a negative puncture . . . . . . . 40

2.3 .5 Bubbling-off tree . . . . . . . . . . . . . 43 
2.3 .6 Estimating Conley-Zehnder indices . . . . . . . . 45

2.4 Transverse foliations . . . . . . . . . . . . . . 50

$2.4 .1 \quad$ Finite energy foliations . . . . . . . . . . . . . 51

2.4 .2 Open book decompositions . . . . . . . . . . . 53

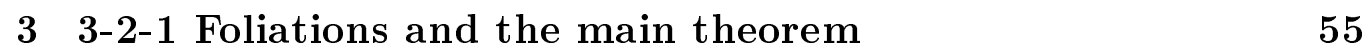

3.1 Main Theorem . . . . . . . . . . . . . . . . 55

3.1 .1 Sketch of the proof of Theorem 3.5 . . . . . . . . 58

3.2 Proof of Proposition 3.6 . . . . . . . . . . . . . . . . 59

3.3 Proof of the main theorem . . . . . . . . . . . . . . 61

3.3.1 A special spanning disk for the orbit $P_{3} \ldots \ldots$. . . 61

3.3 .2 The Bishop family . . . . . . . . . . . . 62

3.3 .3 The case $\pi \cdot d u_{0} \equiv 0$. . . . . . . . . . . . 68

3.3 .4 The case $\int_{\mathbb{D} \backslash \Gamma_{0}} u_{0}^{*} d \lambda>0 \ldots \ldots . \ldots . \ldots 72$

4 Proof of the case $\pi \cdot d u_{0} \equiv 0 \quad 75$

4.1 Foliating a solid torus in the case $\Gamma_{1}=\emptyset \ldots . . . . .7676$

4.2 Foliating a solid torus in the case $\Gamma_{1}=\{0\} \ldots \ldots$. . . . 86

$4.3 \quad$ A cylinder asymptotic to $P_{2}$ and $P_{1} \ldots \ldots \ldots$. . . . . 88

4.3 .1 Limiting behavior . . . . . . . . . . . . . . 91

4.4 A family of cylinders asymptotic to $P_{1}$ and $P_{3} \ldots \ldots$. . . . . 99

4.4.1 Bubbling-off analysis for the family of cylinders . . . . 101

4.4 .2 Proof of Proposition 4.28 . . . . . . . . . . . 108

4.4 .3 The foliation . . . . . . . . . . . . . 115

$5 \quad$ Idea of the proof of case $\int_{\mathbb{D} \backslash \Gamma_{0}} u_{0}^{*} d \lambda>0 \quad 117$

5.1 Gluing . . . . . . . . . . . . . . . . 117

$5.1 .1 \quad$ Pregluing . . . . . . . . . . . . . . . 118

5.2 Idea of the proof of case $\int_{\mathbb{D} \backslash \Gamma_{0}} u_{0}^{*} d \lambda>0 \ldots \ldots . \ldots 120$

\begin{tabular}{|l|l|}
\hline A Proof of Lemma & 3.9 \\
\hline
\end{tabular}

\begin{tabular}{|l|l|}
\hline B Intersection Theory & 131
\end{tabular} 


\section{Introduction}

In this thesis we use the theory of pseudoholomorphic curves in symplectizations to study the existence of finite energy foliations for Reeb flows on $S^{3}$.

We consider $S^{3}$ equipped with the contact form $\lambda=\left.f \lambda_{0}\right|_{S^{3}}$, where $f: S^{3} \rightarrow \mathbb{R} \backslash\{0\}$ is smooth and $\lambda_{0}$ is the standard contact form $\lambda_{0}=$ $\sum_{i=1}^{2} x_{i} d y_{i}-y_{i} d x_{i}$ on $\mathbb{R}^{4}$ with coordinates $\left(x_{1}, x_{2}, y_{1}, y_{2}\right)$. The Reeb vector field $R_{\lambda}$ associated with the contact form $\lambda$ is uniquely determined by the equations

$$
i_{R_{\lambda}} d \lambda=0, \quad \lambda\left(R_{\lambda}\right)=1 .
$$

The flow of $R_{\lambda}$ is called Reeb flow. The symplectization of $\left(S^{3}, \lambda\right)$ is the symplectic manifold $\left(\mathbb{R} \times S^{3}, d\left(e^{a} \lambda\right)\right)$, where $a$ is the coordinate on $\mathbb{R}$. We consider pseudoholomorphic maps $\tilde{u}:(S \backslash \Gamma, j) \rightarrow\left(\mathbb{R} \times S^{3}, \tilde{J}\right)$, where $S$ is a closed Riemann surface, $\Gamma \subset S$ is a finite set, and the almost complex structure $\tilde{J}$ is defined as

$$
\tilde{J} \frac{\partial}{\partial_{a}}=R_{\lambda},\left.\quad \tilde{J}\right|_{\text {ker } \lambda}=J
$$

for any complex structure $J$ on $\operatorname{ker} \lambda$ compatible with $d \lambda$. Due to results of [Hof93] and [HWZ96], if a pseudoholomorphic curve has finite energy, its projection onto $S^{3}$ converges near the ends to periodic orbits of the Reeb flow.

A finite energy foliation $\mathcal{F}$ for the contact manifold $\left(S^{3}, \lambda\right)$ is a collection of $\tilde{J}$-holomorphic curves with uniformly bounded energy, whose images form a smooth foliation of $\mathbb{R} \times S^{3}$. In [HWZ03], H. Hofer, K. Wysocki and E. Zehnder proved that for any nondegenerate contact form $\lambda=f \lambda_{0}$ on $S^{3}$, there exists a finite energy foliation for $\left(S^{3}, \lambda\right)$. The projection of $\mathcal{F}$ onto $S^{3}$ is a singular foliation of $S^{3}$ satisfying the following properties. The singular set consists of finitely many periodic orbits of the Reeb flow, called bindings, having Conley-Zehnder indices in $\{1,2,3\}$. The regular leaves are punctured spheres transverse to the Reeb vector field. Each component of the boundary of the regular leaves is one of the bindings. 


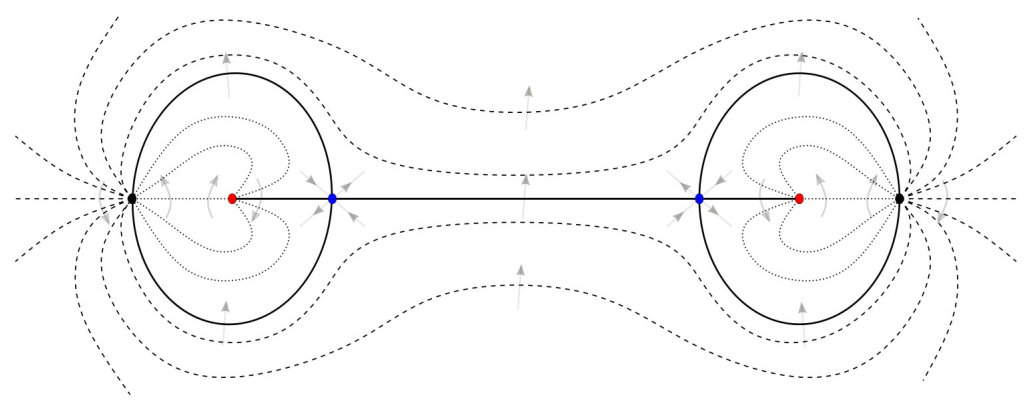

Figure 1: A $3-2-1$ foliation cut by a plane. The dots represent periodic orbits $P_{1}, P_{2}$ and $P_{3}$ with Conley-Zehnder indices respectively 1, 2 and 3 . The bold curves represent two rigid cilinders connecting $P_{2}$ and $P_{3}$, a rigid cylinder connecting $P_{1}$ and $P_{2}$, and a rigid disk with boundary $P_{2}$. The dashed curves represent a family of planes with boundary $P_{3}$. The dotted curves represent a family of cylinders connecting $P_{1}$ and $P_{3}$. The arrows indicate the Reeb flow. The 3 -sphere is viewed as $\mathbb{R}^{3} \cup\{\infty\}$.

If the singular set of the finite energy foliation $\mathcal{F}$ consists of a unique orbit $P$, then $P$ has Conley-Zehnder index 3 and the projection of $\mathcal{F}$ onto $S^{3}$ is an open book decomposition with pages diffeomorphic to disks, where every page is a global surface of section for the Reeb flow. This kind of foliation was obtained in [HWZ98 for Hamiltonian flows on $\mathbb{R}^{4}$ restricted to strictly convex energy levels. Such foliations were also studied in [HSa11, Hry12, Hry14].

The first result on the existence of a finite energy foliation for $S^{3}$ other than the one that projects onto an open book decomposition is due to N. de Paulo e P. Salomão. In dPSa18, the authors study the existence of a finite energy foliation that projects to a singular foliation of $S^{3}$, called 3-2-3 foliation, having an orbit with Conley-Zehnder index 2 and two orbits with Conley-Zehnder index 3 as bindings.

The results of [HWZ98] and [dPSa18] cited above apply to weakly convex contact forms, that is, such that every closed Reeb orbit has Conley-Zehnder index $\geq 2$. Hence, it is natural to ask about the existence of energy levels of Hamiltonians on $\mathbb{R}^{4}$ that admit transverse foliations with index 1 orbits as bindings. The results in this thesis go in the direction of the solution of this question. From assumptions about the Reeb flow and three prescribed Reeb orbits $P_{3}, P_{2}$ and $P_{1}$, with Conley-Zehnder index 3, 2 and 1 respectively, we study the existence of a finite energy foliation that projects onto $S^{3}$ as a singular foliation, that we call $3-2-1$-foliation, having the orbits $P_{3}, P_{2}$ and $P_{1}$ as bindings. See figure 1 .

Our main theorem is the following: 
Theorem. Let $\lambda$ be a nondegenerate tight contact form on $S^{3}$ Let $P_{1}=$ $\left(x_{1}, T_{1}\right), P_{2}=\left(x_{2}, T_{2}\right)$ and $P_{3}=\left(x_{3}, T_{3}\right)$ be simply covered closed Reeb orbits with Conley-Zehnder indices respectively 1, 2 and 3. Assume that the orbits $P_{1}, P_{2}$ and $P_{3}$ are unknotted, $P_{i}$ and $P_{j}$ are not linked for $i \neq j, i, j \in\{1,2,3\}$ and the following conditions hold:

(i) $P_{3}$ spans an embedded disk whose interior is transverse to the Reeb flow;

(ii) $T_{1}<T_{2}<T_{3}<2 T_{1}$;

(iii) $P_{2}$ is the only Reeb orbit with Conley-Zehnder index 2 not linked to $P_{3}$ with period $<T_{3}$;

(iv) $P_{1}$ is the only Reeb orbit with Conley-Zehnder index 1 not linked to $P_{2}$ with period $<T_{2}$;

(v) There is no $C^{1}$-embedding $\Psi: S^{2} \rightarrow S^{3}$ such that $x_{2}\left(T_{2} \cdot\right)=\left.\Psi\right|_{S^{1} \times\{0\}}$ and each hemisphere is a strong transverse section.

Then $P_{1}, P_{2}$ and $P_{3}$ are the binding orbits of a $3-2-1$ foliation.

We also prove that the hypothesis $(v)$ is necessary to the existence of a $3-2-1$ foliation.

The proof of the main theorem depends on a result about gluing of pseudoholomorphic curves that is stated in Chapter 5, but is not proved in this thesis.

\section{Outline of the Thesis}

Chapters 1 and 2 consist of preliminary results. In Chapter 1 we recall some relevant definitions and results about contact geometry and Reeb flows and present the definition and important properties of the asymptotic operator. The subject of Chapter 2 is the theory of pseudoholomorphic curves in symplectizations. We recall results of [HWZ96, HWZ95b, HWZ99b] about asymptotic behavior, algebraic invariants and Fredholm theory. We finish the chapter recalling facts about finite energy foliations.

In chapter 3 we define $3-2-1$ foliations, state the main theorem and give the first steps of the proof. Following the ideas of [Hof93, HWZ95a, HWZ99a, HSa11, we consider a family of pseudoholomorphic disks with boundary in a special embedded disk with spanning $P_{3}$. From a bubbling-off process and exploring the non-compactness of the space of such disks and the properties of its compactification, one can prove the existence of finite energy pseudoholomorphic curves. By our linking hypotheses we have to consider 
two possibilities: either 1 . The boundaries of the family of disks converge to the orbit $P_{3}$ or 2 . The family of disks breaks before approaching $P_{3}$.

In chapter 4 we conclude the proof of case 1 . In chapter 5 we present an idea of the proof of case 2 . 


\section{Chapter 1}

\section{Contact geometry and Reeb dynamics}

\subsection{Contact structures}

Let $M$ be a smooth manifold of dimension $2 n+1$. A contact structure $\xi$ on $M$ is a maximally non-integrable hyperplane distribution on $M$. This is equivalent to the following: $\xi$ can be locally written as the kernel of a 1-form $\lambda$ such that $\lambda \wedge(d \lambda)^{n}$ is a non-vanishing $(2 n+1)$-form. If $\xi$ is a contact structure on $M$, the pair $(M, \xi)$ is called a contact manifold.

If $n$ is odd, the sign of the local form $\lambda \wedge(d \lambda)^{n}$ depends only on $\xi=\operatorname{ker} \lambda$, not on the choice of $\lambda$. So the contact structure induces an orientation on $M$. Assume that $M$ is oriented. We call the contact structure $\xi$ positive if the orientation of $M$ and the orientation given by $\xi$ agree. Otherwise, we say that $\xi$ is negative.

We say that the contact manifold $(M, \xi)$ is co-orientable if the bundle $T M / \xi \rightarrow M$ is trivial. This is equivalent to the existence of a globally defined 1-form $\lambda$ such that $\xi=\operatorname{ker} \lambda$.

A 1-form on $M$ such that $\xi=\operatorname{ker} \lambda$ is a contact structure, that is, $\lambda \wedge(d \lambda)^{n}$ is a volume form on $M$, is called a contact form. If $\lambda$ is a contact form on $M$, we also call the pair $(M, \lambda)$ a contact manifold.

In this thesis we will only consider co-orientable contact manifolds.

The Reeb vector field Let $(M, \lambda)$ be a closed contact manifold. The contact form $\lambda$ defines a vector field $R_{\lambda}$, called Reeb vector field, uniquely defined by

$$
\lambda\left(R_{\lambda}\right) \equiv 1, \quad i_{R_{\lambda}} d \lambda \equiv 0
$$


The Reeb vector field is transverse to the contact structure $\xi$, so that the tangent bundle $T M$ naturally splits into

$$
T M=\mathbb{R} R_{\lambda} \oplus \xi .
$$

If $\left\{\varphi^{t}: M \rightarrow M\right\}_{t \in \mathbb{R}}$ is the flow of $R_{\lambda}$, then $\left(\varphi^{t}\right)^{*} \lambda=\lambda$. Indeed

$$
\frac{d}{d t}\left(\varphi^{t}\right)^{*} \lambda=\left(\varphi^{t}\right)^{*} \mathcal{L}_{R_{\lambda}} \lambda=\left(\varphi^{t}\right)^{*}\left(i_{R_{\lambda}} d \lambda+d i_{R_{\lambda}} \lambda\right)=0, \forall t \in \mathbb{R} .
$$

Consequently, the linearized flow $d \varphi^{t}: T M \rightarrow T M$ leaves the splitting $T M=$ $\mathbb{R} R_{\lambda} \oplus \xi$ invariant.

The restriction of $d \lambda$ to $\xi=\operatorname{ker} \lambda$ is nondegenerate, so that $(\xi, d \lambda)$ is a symplectic vector bundle over $M$. Moreover, the map $d \varphi_{x}^{t}: \xi_{x} \rightarrow \xi_{\varphi^{t}(x)}$ is symplectic with respect to $d \lambda$.

\subsubsection{Hypersurfaces of contact type}

Let $\left(W^{2 n}, \omega\right)$ be a symplectic manifold. A vector field $Y$ defined on an open set of $W$ is called a Liouville vector field if it satisfies $\mathcal{L}_{Y} \omega=\omega$, where $\mathcal{L}_{Y} \omega$ is the Lie derivative of $\omega$ in the direction of $Y$. A compact hypersurface $M$ in $(W, \omega)$ is said to have contact type if there exists a Liouville vector field on a neighborhood of $M$ that is transverse to $M$. Every hypersurface of contact type is a contact manifold, by the following proposition.

Proposition 1.1. Let $M \subset\left(W^{2 n}, \omega\right)$ be a hypersurface of contact type and let $Y$ be a Liouville vector field defined on a neighborhood $U$ of $M$ that is transverse to $M$. Then the 1 -form $\lambda:=\left.i_{Y} \omega\right|_{U}$ satisfies $d \lambda=\omega$ and $\lambda \wedge$ $\left.(d \lambda)^{n-1}\right|_{M}$ is a volume form on $M$.

A proof of Proposition 1.1 can be found in [Gei08, Lemma 1.4.5] or [HSa09, Prop. 1.6].

\section{Star-shaped hypersurfaces on $\mathbb{R}^{2 n}$}

Definition 1.2. A regular hypersurface $S \subset \mathbb{R}^{2 n}$ is said to be star-shaped with respect to the origin if every half-line starting at the origin intersects $S$ transversely in exactly one point.

Consider $\mathbb{R}^{2 n}$ with coordinates $\left(x_{1}, \cdots, x_{n}, y_{1}, \cdots, y_{n}\right)$ equipped with the canonical symplectic form $\omega_{0}=\sum_{i=1}^{n} d x_{i} \wedge d y_{i}$. The vector field $X(z)=\frac{z}{2}$, $z=\left(x_{1}, \cdots, x_{n}, y_{1}, \cdots, y_{n}\right) \in \mathbb{R}^{2 n}$ is a Liouville vector field. Indeed, $i_{X} \omega_{0}=$ 
$\lambda_{0}$, where $\lambda_{0}$ is the Liouville 1 -form

$$
\lambda_{0}=\frac{1}{2} \sum_{i=1}^{n} x_{i} d y_{i}-y_{i} d x_{i} .
$$

Using the Cartan formula we get

$$
\mathcal{L}_{X} \omega_{0}=d i_{X} \omega_{0}+i_{X} d \omega_{0}=d \lambda_{0}=\omega_{0} .
$$

It follows that every hypersurface $S \subset \mathbb{R}^{2 n}$ that is star-shaped with respect to the origin is a contact manifold equipped with the contact form $\left.\lambda_{0}\right|_{S}$.

Let $S$ be a hypersurface $S \subset \mathbb{R}^{2 n}$ that is star-shaped with respect to the origin. For $x \in S^{2 n-1}$, let $f(x) \in \mathbb{R}^{+}$be defined by $\sqrt{f(x)} x \in S$. Define $\psi: S^{2 n-1} \rightarrow S$ by $\psi(x)=\sqrt{f(x)} x$. Then $\psi$ is a diffeomorphism.

Proposition 1.3. The Reeb vector field $X_{\left.\lambda_{0}\right|_{S}}$ on $S$ associated with the contact form $\left.\lambda_{0}\right|_{S}$ is equivalent to Reeb vector field $X_{\left.f \lambda_{0}\right|_{S 2 n-1}}$ on $S^{2 n-1}$ associated with the contact form $\left.f \lambda_{0}\right|_{S^{2 n-1}}$.

Proof. For every $x \in S^{2 n-1}$ and $v \in T_{x} S^{2 n-1}$, we have

$$
\begin{aligned}
\left(\left.\psi^{*} \lambda_{0}\right|_{S}\right)_{x}(v) & =\lambda_{0}\left(d \psi_{x} v\right) \\
& =\omega_{0}\left(\frac{1}{2} \sqrt{f(x)} x,\langle\nabla \sqrt{f}(x), v\rangle x+\sqrt{f(x)} v\right) \\
& =f(x) \omega_{0}\left(\frac{1}{2} x, v\right) \\
& =f(x)\left(\lambda_{0}\right)_{x}(v)
\end{aligned}
$$

It follows that $\psi_{*} X_{\left.f \lambda_{0}\right|_{S} 2 n-1}=X_{\left.\lambda_{0}\right|_{S}}$.

\subsubsection{Symplectization of a contact manifold}

Let $(M, \lambda)$ be a contact manifold. The symplectization of $(M, \lambda)$ is given by the manifold $\mathbb{R} \times M$ equipped with the symplectic form $d\left(e^{a} \lambda\right)$, where $a$ is the coordinate on $\mathbb{R}$ and $\lambda$ is interpreted as the 1 -form on $\mathbb{R} \times M$ given by the pullback of $\lambda$ under the natural projection $\mathbb{R} \times M \rightarrow M$.

The vector field $Y=\frac{\partial}{\partial a}$ is a Liouville vector field, so that $(M, \lambda)$ can be realized as a hypersurface of contact type of its symplectization $(\mathbb{R} \times$ $\left.M, d\left(e^{a} \lambda\right)\right)$.

More generally, if $\phi$ satisfies $\left\{\phi, \phi^{\prime}\right\} \subset C^{\infty}\left(\mathbb{R}, \mathbb{R}^{+}\right)$and the 1 -form $\lambda_{\phi}$ on 
$\mathbb{R} \times M$ is defined by

$$
\lambda_{\phi}(a, x)(h, k)=\phi(a) \lambda(x)(k), \forall(a, x) \in \mathbb{R} \times M,(h, k) \in T_{(a, x)}(\mathbb{R} \times M),
$$

then $\left(\mathbb{R} \times M, d \lambda_{\phi}\right)$ is a symplectic manifold.

\subsubsection{Hamiltonian dynamics}

A Hamiltonian system is a triple $(W, \omega, H)$ where $(W, \omega)$ is a symplectic manifold and $H: W \rightarrow \mathbb{R}$ is a smooth function, that is called a Hamiltonian function. The Hamiltonian vector field associated with $(W, \omega, H)$ is the vector field defined on $W$ by

$$
i_{X_{H}} \omega=-d H .
$$

The vector field $X_{H}$ is well defined because $\omega$ is non-degenerate.

Let $x: \mathbb{R} \rightarrow W$ be a solution of the equation

$$
\dot{x}=X_{H}(x) .
$$

Then the Hamiltonian function is constant along $x$. Indeed,

$$
\frac{d}{d t} H(x(t))=d H\left(X_{H}(x(t))\right)=-\omega\left(X_{H}, X_{H}\right)=0 .
$$

Therefore all the trajectories lie in energy levels of the Hamiltonian function.

Let $S=H^{-1}(e)$ be a regular energy level of $H$. Assume that $S \subset(W, \omega)$ is a hypersurface of contact type. Racall that for a Liouville field $Y$ defined on a neighborhood of $S$ and transverse to $S$, the form $\lambda=\left.i_{Y} \omega\right|_{S}$ is a contact form on $S$. Hence $\left.X_{H}\right|_{S}$ and the Reeb vector field $R_{\lambda}$ lie in the kernel of $\left.\omega\right|_{S}$. Since ker $\left.\omega\right|_{S}$ is 1-dimensional, there exists a function $a: S \rightarrow \mathbb{R} \backslash\{0\}$ such that $\left.X_{H}\right|_{S}(x)=a(x) R_{\lambda}(x), \forall x \in S$. Thus, the trajectories of $\left.X_{H}\right|_{S}$ and $R_{\lambda}$ are the same, modulo reparemetrization.

\subsubsection{Classification of contact structures in dimension 3}

Let $\left(M^{3}, \xi\right)$ be a contact manifold of dimension 3. An embedded disk $D \subset M$ satisfying

$$
T \partial D \subset \xi \text { and } T_{p} D \neq \xi_{p}, \forall p \in \partial D
$$

is called an overtwisted disk.

The contact structure $\xi$ on $M$ is called overtwisted if it admits an overtwisted disk. Otherwise, $\xi$ is called tight. If $\xi=\operatorname{ker} \lambda$ globally, we call the 
contact form overtwisted (tight) if $\xi=\operatorname{ker} \lambda$ is overtwisted (tight).

In this thesis, we will deal with co-oriented tight contact structures on $S^{3}$.

Consider $\mathbb{R}^{4}$ with coordinates $\left(x_{1}, x_{2}, y_{1}, y_{2}\right)$. The Liouville 1 -form

$$
\lambda_{0}=\frac{1}{2} \sum_{i=1}^{2} x_{i} d y_{i}-y_{i} d x_{i}
$$

restricts to a contact form on $S^{3}$. By a result of Bennequim [Ben83, the contact structure $\xi_{0}=\operatorname{ker}\left(\left.\lambda_{0}\right|_{S^{3}}\right)$ is tight. By a Theorem of Eliashberg [Eli92], $\xi_{0}=\operatorname{ker}\left(\left.\lambda_{0}\right|_{S^{3}}\right)$ is the only positive tight contact structure on $S^{3}$, up to diffeomorphism.

\subsection{Reeb orbits}

Let $(M, \lambda)$ be a closed contact manifold of dimension 3 . We call a pair $P=(x, T)$, where $x: \mathbb{R} \rightarrow S^{3}$ is a periodic trajectory of $\dot{x}(t)=R_{\lambda}(x(t))$ and $T>0$ is a period of $x$, a closed Reeb orbit. We identify $P=(x, T)$ with the element of $\frac{C^{\infty}(\mathbb{R} / \mathbb{Z}, M)}{\mathbb{R} / \mathbb{Z}}$ induced by the loop

$$
x_{T}: \frac{\mathbb{R}}{\mathbb{Z}} \rightarrow M, \quad x_{T}(t)=x(T t),
$$

where the quotient is relative to the translations $t \mapsto x_{T}(t+c)$. The set of periodic Reeb orbits will be denoted by $\mathcal{P}(\lambda)$. If $T$ is the minimal positive period of $x$, we call $P$ simply covered or prime. If $m \geq 1$ is an integer, the $m^{t h}$ iterate of $P$ will be denoted by $P^{m}:=(x, m T)$.

Assume $M=S^{3}$. The self-linking number $\operatorname{sl}(L)$ of a knot $L \subset S^{3}$ transverse to $\xi$ is defined as follows. Consider $S^{3}$ oriented by $\lambda \wedge d \lambda$, choose a Seifert surface ${ }^{1} \Sigma$ for $L$ and a smooth nonvanishing section $Z$ of $\left.\xi\right|_{\Sigma} \rightarrow \Sigma$. The section $Z$ is used to slightly perturb $L$ to another knot $L_{\epsilon}=\left\{\exp _{x}\left(\epsilon Z_{x}\right) \mid x \in L\right\}$ transverse to $\xi$ and $\Sigma$. A choice of orientation for $\Sigma$ induces orientations of $L$ and $L_{\epsilon}$. The self-linking number $\operatorname{sl}(L)$ of $L$ is the oriented intersection number $L_{\epsilon} \cdot \Sigma$ of $L_{\epsilon}$ and $\Sigma$. It is independent of the choices of $Z$ and $\Sigma$. Proofs of these facts can be found in Gei08. If $P=(x, T)$ is a closed Reeb orbit, we define its self-linking number by $\operatorname{sl}(P)=\operatorname{sl}(x(\mathbb{R}))$.

\footnotetext{
${ }^{1}$ A Seifert surface for $L$ is an orientable, embedded, connected and compact surface $\Sigma$ such that $L=\partial \Sigma$.
} 
The orbit $P=(x, T)$ is called unknotted if $x(\mathbb{R})$ is an unknot ${ }^{2}$ We say that a set of orbits $\cup_{i=1}^{n} P_{i}=\left(x_{i}, T_{i}\right)$ is an unlink if $\cup_{i=1} x_{i T_{i}}$ is an unlink.

We say that two orbits $P$ and $\bar{P}$ are linked if the linking number 3 $\operatorname{lk}\left(x_{T}, \bar{x}_{\bar{T}}\right)$ is nonzero.

We call the orbit $P=(x, T)$ nondegenerate if 1 is not an eigenvalue of $\left.d \varphi^{T}\right|_{\xi_{x(0)}}$. If every orbit $P \in \mathcal{P}(\lambda)$ is nondegenerate, then the contact form $\lambda$ is called nondegenerate.

Periods of Reeb orbits We call a number $T>0$ a period if there exists a $T$-periodic orbit $P \in \mathcal{P}(\lambda)$.

Fix $C>0$. If the contact form $\lambda$ is nondegenerate, then there exists a finite number of orbits in $\mathcal{P}(\lambda)$ with period less that $C$. This is a consequence of the following lemma.

Lemma 1.4. Let $\lambda$ be a nondegenerate contact form on a closed manifold M. Fix $C>0$ and let $(x, T) \in \mathcal{P}(\lambda)$ be a prime orbit satisfying $T \leq C$. Then there exists a neighborhood $V \subset M$ of $x(\mathbb{R})$ such that if $(\bar{x}, \bar{T}) \in \mathcal{P}(\lambda) \backslash$ $\{(x, T)\}$ satisfies $\bar{T} \leq C$, then $\bar{x}(\mathbb{R}) \cap V=\emptyset$. In particular, there exists a finite number of orbits in $\mathcal{P}(\lambda)$ with period $\leq C$.

Proof. Consider a Poincaré section $\mathcal{D}$ at $x(0)$ such that $T_{x(0)} \mathcal{D}=\xi_{x(0)}$. Let $U \subset \mathcal{D}$ be a neighborhood of $x(0)$ such that the first return map

$$
\phi: U \rightarrow \mathcal{D}, \quad \phi(x)=\varphi^{\tau^{*}(x)}(x)
$$

is defined and $\phi(U) \subset U$, so that the iterate $\phi^{k}: U \rightarrow U$ is defined for every $k \geq 1$. Here $\tau^{*}: U \rightarrow \mathbb{R}^{+}$is the first return map defined by $\tau^{*}(x)=$ $\inf \left\{t>0 \mid \varphi^{t}(x) \in \mathcal{D}\right\}$. Computing the derivative of $\phi$ at $x(0)$ and using $T=\tau^{*}(x(0))$, we obtain

$$
d \phi_{x(0)}=\left.d \varphi^{T}\right|_{\xi(x(0))}
$$

Since the orbit $(x, T)$ is nondegenerate, then

$$
d \phi_{x(0)}-i d=\left.d \varphi^{T}\right|_{\xi(x(0))}-i d
$$

\footnotetext{
${ }^{2} \mathrm{~A}$ knot is a copy of $S^{1}$ embedded in $S^{3}$. More generally, a link with $n$ components is a disjoint union of $n$ copies of $S^{1}$ embedded in $S^{3}$. A knot $K$ is called unknot or trivial $k n o t$ if there exists a embedded disk $D \subset S^{3}$ such that $K=\partial D$. A link $L=\bigsqcup_{i=1}^{n} L_{i}$ is called unlink or trivial link if there exist $n$ disjoint embedded disks $D_{1}, \ldots, D_{n}$ in $S^{3}$ such that $\partial D_{i}=L_{i}$.

${ }^{3} \operatorname{lk}\left(x_{T}, \bar{x}_{\bar{T}}\right)=\operatorname{lk}\left(\bar{x}_{\bar{T}}, x_{T}\right)$ and $\operatorname{lk}\left(x_{T}, \bar{x}_{\bar{T}}\right)=0$ if and only if the homology class of $t \in \mathbb{R} / \mathbb{Z} \mapsto x(T t)$ in $H_{1}\left(S^{3} \backslash \bar{x}(\mathbb{R}), \mathbb{Z}\right)$ is zero.

${ }^{4}$ not linked $\nRightarrow$ unlink, but unlink $\Rightarrow$ not linked.
} 
is an isomorphism. Using the inverse function theorem, we conclude that there exists a neighborhood $U_{1} \subset U$ of $x(0)$ such that $x(0)$ is the only fixed point of $\phi$ in $U$. For any $k>1$, we have $d \phi^{k} x(0)=\left.d \varphi^{n T}\right|_{\xi(x(0))}$. Since the orbit $(x, n T)$ is nondegenerate, then $d \phi^{k} x(0)-i d=\left.d \varphi^{n T}\right|_{\xi(x(0))}-i d$ is an isomorphism, so that $x(0)$ is the only fixed point of $\phi^{k}$ in a neighborhood $U_{k} \subset U$ of $x(0)$. Fix $N \in \mathbb{N}$ such that $N T>C$ and define $\mathcal{U}=\bigcap_{n=1}^{2 N} U_{k}$. Then $x(0)$ is the only periodic point of $\phi$ in $\mathcal{U}$ with period $\leq 2 N$. Shrinking $\mathcal{U}$ if necessary, we can assume that $\phi(\mathcal{U}) \subset \mathcal{U}$ and for every $y \in \mathcal{U}$,

$$
\left|\tau^{*}(y)-\tau^{*}(x(0))\right|<\frac{T}{2} .
$$

Let $(\bar{x}, \bar{T}) \in \mathcal{P}(\lambda) \backslash\{(x, T)\}$ be a prime orbit such that $\bar{x}(0) \in \mathcal{U}$. Then $\bar{x}(0)$ is a periodic point of $\phi$ with period $M>2 N$. It follows that $\mid \tau^{*}\left(\phi^{k}(\bar{x}(0))\right)-$ $\tau^{*}(x(0)) \mid<\frac{T}{2}$, for $k=0, \ldots, M-1$. Since $\sum_{k=0}^{M-1} \tau^{*}\left(\phi^{k}(\bar{x}(0))\right)=\bar{T}$, we have

$$
|\bar{T}-M T| \leq \sum_{k=0}^{M-1}\left|\tau^{*}\left(\phi^{k}(\bar{x}(0))\right)-T\right|<M \frac{T}{2} .
$$

It follows that

$$
C<N T<M \frac{T}{2}=M T-M \frac{T}{2} \leq \bar{T} .
$$

We have proved that if an orbit $(\bar{x}, \bar{T}) \in \mathcal{P}(\lambda) \backslash\{(x, T)\}$ satisfy $\bar{T} \leq C$, then $\bar{x}(\mathbb{R})$ does not intersect the section $\mathcal{U}$. Let $\mathcal{V}$ be a neighborhood of $x(\mathbb{R})$ in $M$ such that any orbit intersecting the neighborhood $\mathcal{V}$ also intersects the section $\mathcal{U}$. Then any orbit $(\bar{x}, \bar{T}) \in \mathcal{P}(\lambda) \backslash\{(x, T)\}$ satisfying $\bar{T} \leq C$ satisfies $\bar{x}(\mathbb{R}) \cap \mathcal{V}=\emptyset$.

Thus, if $\lambda$ is nondegenerate, we can define $\sigma(C)$ as any real number satisfying

$$
0<\sigma(C)<\min \left\{T^{\prime},\left|T^{\prime}-T^{\prime \prime}\right|: T^{\prime} \neq T^{\prime \prime} \text { periods }, T^{\prime}, T^{\prime \prime} \leq C\right\} .
$$

The number $\sigma(C)$ will be important later in Chapters 2 and 4 .

\subsubsection{The Conley-Zehnder index}

An axiomatic definition Let $S p(n)$ denote the symplectic group in dimension $2 n$. Consider the set $\Sigma^{*}(1)$ of paths $\varphi \in C^{\infty}([0,1], \operatorname{Sp}(1))$ such that $\varphi(0)=I$ and $\operatorname{det}(\varphi(1)-I) \neq 0$.

The Conley-Zehnder index is a map $\mu: \Sigma^{*}(1) \rightarrow \mathbb{R}$ that can be axiomatically characterized as follows. 
Theorem 1.5 ([HWZ03, Theorem 8.1]). There is a unique surjective map $\mu: \Sigma^{*}(1) \rightarrow \mathbb{Z}$, called the Conley-Zehnder index, that satisfies the following axioms 5

(1) The map $s \mapsto \mu\left(\varphi^{s}\right)$ is constant if $\left\{\varphi^{s}\right\}$ is a homotopy of paths in $\Sigma^{*}(1)$;

(2) If $\psi:[0,1] \rightarrow \mathrm{Sp}(1)$ is a smooth loop based at I, then

$$
\mu(\psi \varphi)=2 \operatorname{Maslov}(\psi)+\mu(\varphi), \quad \forall \varphi \in \Sigma^{*}(1)
$$

(3) If $\varphi \in \Sigma^{*}(1)$, then $\mu\left(\varphi^{-1}\right)=-\mu(\varphi)$

(4) If $\varphi(t)=\left(\begin{array}{rr}\cos (\pi t) & -\sin (\pi t) \\ \sin (\pi t) & \cos (\pi t)\end{array}\right)$, then $\mu(\varphi)=1$.

Fix a nondegenerate orbit $P=(x, T) \in \mathcal{P}(\lambda)$. The vector bundle $x_{T}^{*} \xi \rightarrow$ $S^{1}$ becomes a symplectic bundle with the bilinear form $d \lambda$.

Let $\Psi: x_{T}^{*} \xi \rightarrow S^{1} \times \mathbb{R}^{2}$ be a symplectic trivialization and consider the arc of symplectic matrices $\Phi \in \mathbb{C}^{\infty}([0,1], S p(1))$ defined by

$$
\Phi(t)=\left.\Psi_{t} \circ d \varphi^{T t}\right|_{\xi_{x(0)}} \circ \Psi_{0}^{-1}
$$

The $\operatorname{arc} \Phi$ satisfies $\Phi(0)=I$ and, since $P$ is nondegenerate, $\operatorname{det}(\Phi(1)-I) \neq 0$. Thus, $\Phi \in \Sigma^{*}(1)$.

Definition 1.6. We define the Conley-Zehnder index of the orbit $P$ relative to the trivialization $\Psi$ by

$$
\mu(P, \Psi)=\mu(\Phi) \in \mathbb{Z}
$$

This index only depends on the homotopy class ${ }^{6}$ of the trivialization $\Psi$ and we denote $\mu(P,[\Psi])=\mu(P, \Psi)$.

Global trivializations of the tight contact structure $\xi_{0}$ on $S^{3}$ Later on, we will only deal with the tight contact structure $\xi_{0}=\left.\operatorname{ker} \lambda_{0}\right|_{S^{3}}$ on $S^{3}$, that is a trivial symplectic bundle. Here $\lambda_{0}$ is the Liouville 1-form defined

\footnotetext{
${ }^{5}$ The Maslov index is a function that assigns an integer to every path of symplectic matrices $\psi: \frac{\mathbb{R}}{\mathbb{Z}} \rightarrow S p(n)$. This function is homotopy invariant and induces an isomorphism $\pi_{1}(S p(n)) \rightarrow \mathbb{Z}$. Definitions can be found in MS17 or HWZ95b.

${ }^{6}$ Let $E \stackrel{p}{\rightarrow} B$ be a vector bundle of rank $k$. Two trivializations $\Psi, \Phi: E \rightarrow B \times \mathbb{R}^{k}$ are homotopic if there exists a trivialization $F: E \times I \rightarrow(B \times I) \times \mathbb{R}^{k} \cong\left(B \times \mathbb{R}^{k}\right) \times I$ of the vector bundle $E \times I \stackrel{p \times i d}{\longrightarrow} B \times I$ such that, for every $e \in E, F(e, 0)=(\Psi(e))$ and $F(e, 1)=(\Phi(e), 1)$.
} 
in 1.3. Now we show that the Conley-Zehnder index is independent of the choice of global symplectic trivialization of $\xi_{0}$.

Let $\Psi, \Psi^{\prime}: \xi_{0} \rightarrow S^{3} \times \mathbb{R}^{2}$ be two symplectic trivializations of $\xi_{0}$ and let $P \in \mathcal{P}(\lambda)$ be a nondegenerate orbit, where $\lambda$ is a contact form such that $\xi_{0}=\operatorname{ker} \lambda$. Then the trivializations $\Psi: x_{T}^{*} \xi_{0} \rightarrow \mathbb{R} / \mathbb{Z} \times \mathbb{R}^{2}$ and $\Psi^{\prime}: x_{T}^{*} \xi_{0} \rightarrow$ $\mathbb{R} / \mathbb{Z} \times \mathbb{R}^{2}$ are homotopic. This is a consequence of the fact that the orbit $P$ is contractible and of the following lemma.

Lemma 1.7. Let $\phi: \mathbb{D} \rightarrow S^{3}$ be a continuous map. Then any two trivializations of the bundle $\phi^{*} \xi_{0}$ are homotopic.

Proof. Let $\Psi_{1}, \Psi_{2}: \phi^{*} \xi_{0} \rightarrow \mathbb{D} \times \mathbb{R}^{2}$ be two symplectic trivializations of the bundle $\phi^{*} \xi_{0} \stackrel{p}{\rightarrow} \mathbb{D}$. Consider the map $f: \mathbb{D} \rightarrow S p(1)$ defined by $a \mapsto \Psi_{1} \circ$

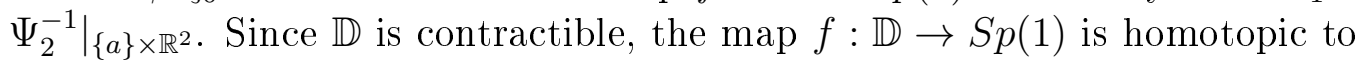
a constant map. Since $S p(1)$ is path connected, any constant map $x \in \mathbb{D} \mapsto$ $c \in S p(1)$ is homotopic to the constant map $x \in \mathbb{D} \mapsto i d \in S p(1)$. Thus, there exists a homotopy $H: \mathbb{D} \times I \rightarrow S p(1)$ between $f: \mathbb{D} \rightarrow S p(1)$ and the constant map $x \in \mathbb{D} \mapsto i d \in S p(1)$. Define $G: \phi^{*} \xi_{0} \times I \rightarrow \mathbb{D} \times \mathbb{R}^{2} \times I$ by

$$
G(e, t)=\left(p(e),\left.H(p(e), t) \cdot \Psi_{2}\right|_{p^{-1}(p(e))}(e), t\right) \text {, for }(e, t) \in \phi^{*} \xi_{0} \times I \text {. }
$$

Then $G$ defines a homotopy between the trivializations $\Psi_{1}$ and $\Psi_{2}$. In fact, $G$ is a bundle morphism with inverse given by

$$
\begin{aligned}
& G^{-1}(x, v, t)=\left(\left.\Psi_{2}^{-1}\right|_{\{x\} \times \mathbb{R}^{2}}\left(H(x, t)^{-1} \cdot v\right), t\right), \text { for }(x, v, t) \in \mathbb{D} \times \mathbb{R}^{2} \times I, \\
& \begin{aligned}
G(e, 0) & =\left(p(e),\left.H(p(e), 0) \cdot \Psi_{2}\right|_{p^{-1}(p(e))}(e), 0\right) \\
& =\left(p(e),\left.\left.\left(\Psi_{1} \circ \Psi_{2}^{-1}\right)\right|_{\{p(e)\} \times \mathbb{R}^{2}} \cdot \Psi_{2}\right|_{p^{-1}(p(e))}(e), 0\right)=\Psi_{1}(e), \forall e \in \phi^{*} \xi_{0}
\end{aligned}
\end{aligned}
$$

and

$$
\begin{aligned}
G(e, 1) & =\left(p(e),\left.H(p(e), 1) \cdot \Psi_{2}\right|_{p^{-1}(p(e))}(e), 1\right) \\
& =\left(p(e),\left.i d \cdot \Psi_{2}\right|_{p^{-1}(p(e))}(e), 1\right)=\Psi_{2}(e), \forall e \in \phi^{*} \xi_{0} .
\end{aligned}
$$

A geometric description of the Conley-Zehnder index Following [HWZ03], we present a geometric construction for the index $\mu: \Sigma^{*}(1) \rightarrow \mathbb{Z}$.

Fix $\Phi \in \Sigma^{*}(1)$. Let $z \in \mathbb{C} \backslash\{0\}$ and let $\theta:[0,1] \rightarrow \mathbb{R}$ be a continuous argument for $z(t):=\Phi(t) z$, that is, $e^{2 \pi \theta(t)}=\frac{z(t)}{|z(t)|}, \forall 0 \leq t \leq 1$.

Define the winding number of $z(t)=\Phi(t) z$ by

$$
\Delta(z)=\theta(1)-\theta(0) \in \mathbb{R}
$$


and the winding interval of the arc $\Phi$ by

$$
I(\Phi)=\{\Delta(z) \mid z \in \mathbb{C} \backslash\{0\}\} .
$$

It is proved in [HWZ03] that the length of this interval is strictly smaller than $\frac{1}{2}$. Thus, the winding interval either lies between two consecutive integers or contains precisely one integer. Define

$$
\mu(\Phi)=\left\{\begin{array}{l}
2 k+1, \text { if } I(\Phi) \subset(k, k+1) \\
2 k, \text { if } k \in I(\Phi)
\end{array}\right.
$$

It is proved in HK99 that $\Phi \mapsto \mu(\Phi)$ satisfies the assumptions of Theorem 1.5. Thus, it agrees with the Conley-Zehnder index.

Recall that the spectrum of a matrix $A \in S p(1)$ with $\operatorname{det}(A-I) \neq 0$ satisfies the following: either $\sigma(A)=\{\lambda, \bar{\lambda}\} \subset S^{1} \backslash\{1\}, \sigma(A)=\left\{\lambda, \lambda^{-1}\right\} \subset$ $(0, \infty) \backslash\{1\}$ or $\sigma(A)=\left\{\lambda, \lambda^{-1}\right\} \subset(-\infty, 0)$.

Definition 1.8. We say that an arc $\Phi \in \Sigma^{*}(1)$ is hyperbolic positive if $\sigma(\Phi(1)) \subset(0, \infty) \backslash\{1\}$, hyperbolic negative if $\sigma(\Phi(1)) \subset(-\infty, 0) \backslash\{-1\}$, or elliptic if the eigenvalues are in $S^{1} \backslash\{1\}$.

Let $z \in \mathbb{C} \backslash\{0\}$. The winding number $\Delta(z)$ is an integer if and only if there exists $\lambda>0$ such that $\Phi(1) z=\lambda z$. Thus, an arc is positive hyperbolic if and only if it has even index. For a negative hyperbolic arc, there exists $z \in \mathbb{C} \backslash\{0\}$ such that $\Phi(1) z=-\lambda z$, for some $\lambda>0$, so that $\Delta(z)=k+\frac{1}{2}$, for some $k \in \mathbb{Z}$. Thus $\mu(\Phi)=2 k+1$. Elliptic arcs also necessarily have odd indices.

Let $P=(x, T) \in \mathcal{P}(\lambda)$ be nondegenerate and assume that for all $n \in \mathbb{Z}$, the orbit $P^{n}=(x, n T)$ is nondegenerate. Fix a symplectic trivialization $\Psi: x_{T}^{*} \xi \rightarrow S^{1} \times \mathbb{R}^{2}$. The trivialization $\Psi$ induces a trivialization of $x_{n T}^{*} \xi$ that we also denote by $\Psi$. The following Lemma is a consequence of the properties of the Conley-Zehnder index proved in [HWZ03].

Lemma 1.9. Assume $\lambda$ is nondegenerate, let $P=(x, T)$ be a closed Reeb orbit and fix positive integers $1 \leq l \leq k$. The following assertions hold.

(1) If $\mu\left(P^{k}, \Psi\right)=1$, then $\mu\left(P^{l}, \Psi\right)=1$;

(2) $\mu\left(P^{k}, \Psi\right) \leq 0 \Longleftrightarrow \mu\left(P^{l}, \Psi\right) \leq 0$;

(3) If $P$ is a hyperbolic orbit, then $\mu\left(P^{l}, \Psi\right)=l \mu(P, \Psi)$;

(4) If $\mu\left(P^{k}, \Psi\right)=2$, then $k, l$ and $\mu\left(P^{l}, \Psi\right)$ belong to $\{1,2\}$ and $P$ is hyperbolic. If $l=1$ and $k=2$, then $\mu(P, \Psi)=1$. 


\subsection{The asymptotic operator}

Let $E \stackrel{\pi}{\rightarrow} X$ be a smooth vector bundle. A complex structure $J$ on $E$ is a smooth fiberwise linear map satisfying $J_{x}^{2}=-I$ on $\pi^{-1}(x)$, for all $x \in X$.

Definition 1.10. Let $(E \stackrel{\pi}{\rightarrow} X, \omega)$ be a symplectic vector bundle and let $J$ be a smooth complex structure on $E$. $J$ is said to be $\omega$-compatible if

$$
\omega_{x}\left(\cdot, J_{x} \cdot\right)
$$

defines a positive definite inner product on $E_{x}$, for all $x \in X$. This is equivalent to requiring that for all $x \in X, \omega_{x}\left(J_{x} \cdot, J_{x} \cdot\right)=\omega_{x}$ and $\omega_{x}\left(v, J_{x} v\right)>0$, for $0 \neq v \in \pi^{-1}(x)$.

The set $\mathcal{J}(E, \omega)$ of $\omega$-compatible complex structures on $(E, \omega)$ is non empty and contractible. A proof of this fact can be found in [MS17].

Definition of the asymptotic operator Let $(M, \lambda)$ be a closed contact manifold of dimension 3 and let $R$ be the associated Reeb vector field. Let $P=(x, T)$ be a closed Reeb orbit and let $h$ be a vector field along $x_{T}$ : $S^{1}=\mathbb{R} / \mathbb{Z} \rightarrow M$, that is, $h: S^{1} \rightarrow T M$ is a smooth function satisfying $h(t) \in T_{x(T t)} M, \forall t \in S^{1}$. We can define the Lie derivative $\mathcal{L}_{R} h$ of $h$ by

$$
\mathcal{L}_{R} h(t)=\left.\frac{d}{d s}\right|_{s=0} d \varphi^{-s}(x(T t+s)) h\left(t+\frac{s}{T}\right),
$$

where $\varphi^{t}$ is the flow of $R$. Note that it is the same as defining the Lie derivative of $h$ as the Lie derivative of any extension of $h$ to a neighborhood of $h(t)$ in $M$. Let $\nabla$ be a symmetric (torsion-free) connection on $T M$. We can use $d x_{T}(t) \partial_{t}=T R\left(x_{T}(t)\right)$ to write

$$
T \mathcal{L}_{R} h=\mathcal{L}_{T R} h=[T R, \tilde{h}]=\nabla_{T R} h-\nabla_{h} T R=\nabla_{t} h-T \nabla_{h} R,
$$

where $\tilde{h}$ is any extension of $h$ and $\nabla_{t}$ is the covariant derivative along $x_{T}$. We conclude that the differential operator $\nabla_{t} \cdot-T \nabla \cdot R$ maps sections of $x_{T}^{*} \xi$ to sections of $x_{T}^{*} \xi$ and is independent of the choice of symmetric connection.

Choosing some $J \in \mathcal{J}(\xi, d \lambda)$, we associate to the orbit $(x, T)$ the unbounded differential operator 7

$$
A_{P, J}: \mathcal{D}\left(A_{P, J}\right)=W^{1,2}\left(S^{1}, x_{T}^{*} \xi\right) \subset L^{2}\left(S^{1}, x_{T}^{*} \xi\right) \rightarrow L^{2}\left(S^{1}, x_{T}^{*} \xi\right)
$$

\footnotetext{
${ }^{7}$ We say that $Y \in W^{1, p}\left(x_{P}^{*} \xi\right)$ (or $\left.Y \in L\left(x_{T}^{*} \xi\right)\right)$ if in any trivialization $\Psi: x_{T}^{*} \xi \rightarrow$ $S^{1} \times \mathbb{R}^{2}$, where $Y$ takes the form $Y=\left(y_{1}(t), y_{2}(t)\right)$, the function $\left(y_{1}, y_{2}\right): S^{1} \rightarrow \mathbb{R}^{2}$ is in $W^{1, p}\left(S^{1}, \mathbb{R}^{2}\right)\left(\right.$ or $\left.L^{2}\left(S^{1}, \mathbb{R}^{2}\right)\right)$.
} 
defined by

$$
A_{P, J}(h)=-J\left(\nabla_{t} h-T \nabla_{h} R\right)
$$

Definition 1.11 (Asymptotic operator). The operator $A_{P, J}$ defined by (1.8) is called the asymptotic operator associated to the orbit $P$ and the complex structure $J$.

\section{The asymptotic operator in unitary trivializations}

Definition 1.12. A unitary trivialization of the bundle $\left(x_{T}^{*} \xi, d \lambda, J\right)$ is a trivialization $\Psi: x_{T}^{*} \xi \rightarrow S^{1} \times \mathbb{R}^{2}$ of $x_{T}^{*} \xi$ satisfying

$$
\left\{\begin{array}{l}
d \lambda=\Psi^{*} d x_{1} \wedge d x_{2} \\
J_{0} \Psi=\Psi J
\end{array}\right.
$$

where $\left(x_{1}, x_{2}\right)$ are coordinates on $\mathbb{R}^{2}$ and $J_{0}=\left[\begin{array}{rr}0 & -1 \\ 1 & 0\end{array}\right]$.

Proposition 1.13. In any unitary trivialization $\Psi$ of $\left(x_{T}^{*} \xi, d \lambda, J\right)$, the operator $A_{P, J}$ takes the form

$$
L_{S}:=-J_{0} \frac{d}{d t}-S(t)
$$

where $S(t)$ is a path of symmetric matrices given by $S(t)=-J_{0} \dot{\phi}(t) \phi(t)^{-1}$, and $\phi(t)$ is the linearized flow restricted to $\xi$ in the trivialization $\Psi$.

Proof. Consider the path of symplectic matrices

$$
\phi(t)=\left.\Psi_{t} \circ d \varphi^{T t}\right|_{\xi_{x(0)}} \circ \Psi_{0}^{-1},
$$

where $\varphi^{t}$ is the Reeb flow. Note that for every $t \in \mathbb{R}$, the matrix $S(t)=$ $-J_{0} \dot{\phi}(t) \phi(t)$ is symmetric. Indeed, since $\phi(t)$ is symplectic, we have the identity

$$
J_{0} \dot{\phi}(t)^{T} J_{0}=\frac{d}{d t}\left(J_{0} \phi(t)^{T} J_{0}\right)=\frac{d}{d t}\left(-\phi(t)^{-1}\right)=\phi(t)^{-1} \dot{\phi}(t) \phi(t)^{-1}
$$

Using (1.10) and the identity $\left(\phi(t)^{-1}\right)^{T}=-J_{0} \phi(t) J_{0}$, we get

$$
\begin{aligned}
S(t)^{T} & =\left(-J_{0} \dot{\phi}(t) \phi(t)^{-1}\right)^{T}=\left(\phi(t)^{-1}\right)^{T} \dot{\phi}(t)^{T} J_{0} \\
& =\left(\phi(t)^{-1}\right)^{T}\left(-J_{0}\right) \phi^{-1}(t) \dot{\phi}(t) \phi^{-1}(t)=-J_{0} \dot{\phi}(t) \phi^{-1}(t)=S(t) .
\end{aligned}
$$


Along $x(T t), T_{x(T t)} M=\operatorname{Span}\left\{T R, \Psi_{t}^{-1} e_{1}, \Psi_{t}^{-1} e_{2}\right\}$, where $e_{1}=(1,0)$ and $e_{2}=(0,1)$. In this coordinates for $x_{T}^{*} T M$, the linearized flow can be represented by a $3 \times 3$ matrix written in blocks

$$
d \varphi_{T t}(x(0))=\left[\begin{array}{rr}
1 & 0 \\
0 & \phi(t)
\end{array}\right] .
$$

Using the identity

$$
\frac{d}{d t} d \varphi_{T t}(x(0))=T\left(d R \circ \varphi_{T t}\right) d \varphi_{T t}(x(0)),
$$

we conclude that $d R(x(T t))$ can be written in the same coordinates above as

$$
\begin{aligned}
\operatorname{TdR}(x(T t)) & =\frac{d}{d t} d \varphi_{T t}(x(0)) \circ d \varphi_{T t}(x(0))^{-1} \\
& =\left[\begin{array}{rr}
0 & 0 \\
0 & \dot{\phi}(t)
\end{array}\right]\left[\begin{array}{rr}
1 & 0 \\
0 & \phi(t)^{-1}
\end{array}\right]=\left[\begin{array}{rr}
0 & 0 \\
0 & \dot{\phi}(t) \phi^{-1}(t)
\end{array}\right] .
\end{aligned}
$$

Now we compute $\Psi \circ A_{P, J} \circ \Psi^{-1}$. Let $n: S^{1} \rightarrow \mathbb{R}^{2}, n(t)=\left(a_{1}(t), a_{2}(t)\right)$ be a smooth function. Let $\left(t, x_{1}, x_{2}\right)$ be coordinates in a neighborhood of $x(T t)$ in $M$ such that $x(T t)=(t, 0,0), \frac{\partial}{\partial t}(t, 0,0)=T R, \frac{\partial}{\partial x_{1}}(t, 0,0)=\Psi^{-1} e_{1}$ and $\frac{\partial}{\partial x_{2}}(t, 0,0)=\Psi^{-1} e_{2}$. Then

$$
\begin{aligned}
\Psi \circ A_{P, J} \circ \Psi^{-1}(n)(t) & =\Psi\left[-J\left(\nabla_{t} a_{k}(t) \frac{\partial}{\partial x_{k}}-\nabla_{a_{k}(t) \frac{\partial}{\partial x_{k}}} T R\right)(t, 0,0)\right] \\
& =-J_{0} \Psi\left[\dot{a}_{k}(t) \frac{\partial}{\partial x_{k}}+a_{k}(t) \nabla_{T R} \frac{\partial}{\partial x_{k}}-a_{k}(t) \nabla_{\frac{\partial}{\partial x_{k}}} T R\right](t, 0,0) \\
& =-J_{0} \dot{n}(t)-J_{0} \Psi\left[a_{k}(t)\left(\nabla_{\frac{\partial}{\partial t}} \frac{\partial}{\partial x_{k}}-\nabla_{\frac{\partial}{\partial x_{k}}} T R\right)(t, 0,0)\right]
\end{aligned}
$$

We write $T R\left(t, x_{1}, x_{2}\right)$ in coordinates near $x(T t)$ as

$$
T R\left(t, x_{1}, x_{2}\right)=r_{0}\left(t, x_{1}, x_{2}\right) \frac{\partial}{\partial t}+r_{1}\left(t, x_{1}, x_{2}\right) \frac{\partial}{\partial x_{1}}+r_{2}\left(t, x_{1}, x_{2}\right) \frac{\partial}{\partial x_{2}},
$$

so that $T R(t, 0,0)=\frac{\partial}{\partial t}$. Using these coordinates and equation (1.11), we obtain

$$
\nabla_{\frac{\partial}{\partial x_{k}}} T R(t, 0,0)=\frac{\partial r_{1}}{\partial x_{k}} \frac{\partial}{\partial x_{1}}+\frac{\partial r_{2}}{\partial x_{k}} \frac{\partial}{\partial x_{2}}+\nabla_{\frac{\partial}{\partial x_{k}}} \frac{\partial}{\partial t} .
$$


Since $\nabla$ is symmetric, we conclude that

$$
\begin{aligned}
\Psi \circ A_{P, J} \circ \Psi^{-1}(n)(t) & =-J_{0} \dot{n}(t)+J_{0} \Psi\left(a_{k}(t) \frac{\partial r_{i}}{\partial x_{k}} \frac{\partial}{\partial x_{i}}\right) \\
& =-J_{0} \dot{n}(t)+J_{0}\left(\pi_{\xi} d R \pi_{\xi}\right)(t, 0,0) n(t) \\
& =-J_{0} \dot{n}(t)+J_{0} \dot{\phi}(t) \phi(t)^{-1} n(t)
\end{aligned}
$$

Properties of the operator $\boldsymbol{L}_{S}$ The unbounded operator

$$
L_{S}: \mathcal{D}\left(L_{S}\right)=W^{1,2}\left(S^{1}, \mathbb{R}^{2}\right) \subset L^{2}\left(S^{1}, \mathbb{R}^{2}\right) \rightarrow L^{2}\left(S^{1}, \mathbb{R}^{2}\right)
$$

is self-adjoint. Its spectrum consists of real eigenvalues which only accumulate at $+\infty$ and $-\infty .8$

If $n(t)$ is an eigenfunction of $L_{S}$ with corresponding eigenvalue $\lambda \in \mathbb{R}$ and $n(t)$ is not identically zero in $L^{2}\left(S^{1}, \mathbb{R}^{2}\right)$, then $n(t)$ solves the linear first order differential equation

$$
-J_{0} \dot{n}(t)-S(t) n(t)=\lambda n(t), n(t+1)=n(t) .
$$

Hence $n(t) \neq 0$ for all $t \in S^{1}$. It follows that $n(t)$ has a well defined winding number given by

$$
\operatorname{wind}(n, \lambda):=\operatorname{deg}\left(t \mapsto \frac{n(t)}{\|n(t)\|}\right) \in \mathbb{Z}
$$

The following properties about this winding number are proved in [HWZ95b].

Proposition 1.14. HWZ95b]

- Given nonzero eigenfunctions $x(t)$ and $y(t)$ associated to the same eigenvalue $\lambda$, we have

$$
\operatorname{wind}(x, \lambda)=\operatorname{wind}(y, \lambda)
$$

\footnotetext{
${ }^{8}$ It is easy to see that $L_{S}$ is symmetric with respect to the usual inner product in $L^{2}\left(S^{1}, \mathbb{R}^{2}\right)$, that is, $\mathcal{D}\left(L_{S}\right)$ is dense and $\int_{S}^{1}\left\langle L_{S} x(t), y(t)\right\rangle d t=\int_{S}^{1}\left\langle x(t), L_{S} y(t)\right\rangle$, for all $x, y \in$ $W^{1,2}\left(S^{1}, \mathbb{R}^{2}\right)$. Since $L_{S}$ is symmetric and surjective, then $L_{S}$ is self-adjoin (see Proposition 8.3 in Tay11 $)$. Also, $L_{S}$ is a bijection, because $0=\mathcal{R}\left(L_{S}\right)^{\perp}=\operatorname{ker} L_{S}^{*}=\operatorname{ker} L_{S}$. Since $L_{S}$ is symmetric and bijective, $L_{S}^{-1}$ is symmetric. By Hellinger-Toeplitz Theorem, $L_{S}^{-1}$ is bounded. Since the embedding $W^{1,2}\left(S^{1}, \mathbb{R}^{2}\right) \rightarrow L^{2}\left(S^{1}, \mathbb{R}^{2}\right)$ is compact, it follows that $L_{S}^{-1}$ is a compact self-adjoint operator. Applying the Spectral Theorem to $L_{S}^{-1}$, we conclude that the spectrum of $L_{S}$ consists of real eigenvalues which only accumulate at $+\infty$ and $-\infty$.
} 
so that we can define $\operatorname{wind}(\lambda):=\operatorname{wind}(x, \lambda)$, for any eigenfunction associated to $\lambda$.

- If $\lambda \neq \mu \in \sigma\left(L_{S}\right)$ satisfy $\operatorname{wind}(\lambda)=\operatorname{wind}(\mu)$ and $x(t), y(t)$ are nonvanishing $\lambda, \mu$-eigenfunctions, respectively, then $x(t), y(t)$ are pointwise linearly independent.

- Given $k \in \mathbb{Z}$, there exists precisely two eigenvalues $\lambda, \mu \in \sigma\left(L_{S}\right)$, counting multiplicities, such that $\operatorname{wind}(\lambda)=\operatorname{wind}(\mu)=k$

- If $\lambda, \mu \in \sigma\left(L_{S}\right)$ and $\lambda \leq \mu$, then $\operatorname{wind}(\lambda) \leq \operatorname{wind}(\mu)$.

Properties of the asymptotic operator $\boldsymbol{A}_{\boldsymbol{P}, \boldsymbol{J}} \quad$ Given $\Psi: x_{T}^{*} \xi \rightarrow S^{1} \times \mathbb{R}^{2}$ a trivialization and $t \mapsto Y(t)$ a section such that $Y(t) \neq 0$ for all $t \in S^{1}$, we define

$$
\operatorname{wind}(Y, \Psi):=\operatorname{deg}\left(t \mapsto \frac{\Psi(Y(t))}{\|\Psi(Y(t))\|}\right) .
$$

This definition just depends on the homotopy class of the trivialization $\Psi$, and we denote $\operatorname{wind}(Y,[\Psi])=\operatorname{wind}(Y, \Psi)$, for any $\Psi$ in class $[\Psi]$.

The properties of the operator $L_{S}$ discussed above imply similar properties for the asymptotic operator $A_{P, J}$, that we summarize below.

Proposition 1.15. The unbounded operator $A_{P}$ has discrete real spectrum accumulating only at $\pm \infty$. Fix $[\Psi]$ a homotopy class of unitary trivializations. Then

- Given nonzero eigensections $\eta_{1}(t)$ and $\eta_{2}(t)$ associated to the same eigenvalue $\lambda$, we have

$$
\operatorname{wind}\left(\eta_{1},[\Psi]\right)=\operatorname{wind}\left(\eta_{2},[\Psi]\right)
$$

so that we can define $\operatorname{wind}(\lambda,[\Psi]):=\operatorname{wind}(\eta,[\Psi])$, for any eigensection $\eta$ associated to $\lambda$.

- If $\lambda \neq \mu \in \sigma\left(A_{P, J}\right)$ satisfy $\operatorname{wind}(\lambda,[\Psi])=\operatorname{wind}(\mu,[\Psi])$ and $\eta(t), \nu(t)$ are non-vanishing $\lambda, \mu$-eigensections, respectively, then $\eta(t), \nu(t)$ are pointwise linearly independent.

- Given $k \in \mathbb{Z}$, there exists precisely two eigenvalues $\lambda, \mu \in \sigma\left(A_{P, J}\right)$, counting multiplicities, such that $\operatorname{wind}(\lambda,[\Psi])=\operatorname{wind}(\mu,[\Psi])=k$

- If $\lambda, \mu \in \sigma\left(A_{P, J}\right)$ and $\lambda \leq \mu$, then $\operatorname{wind}(\lambda,[\Psi]) \leq \operatorname{wind}(\mu,[\Psi])$.

- $0 \notin \sigma\left(A_{P, J}\right)$ if and only if the orbit $P=(x, T)$ is nondegenerate. 
The Conley-Zehnder index Define

$$
\begin{gathered}
\nu_{P}^{n e g}=\max \left\{\nu<0 \mid \nu \text { is an eigenvalue of } A_{P}\right\} \\
\nu_{P}^{\text {pos }}=\min \left\{\nu \geq 0 \mid \nu \text { is an eigenvalue of } A_{P}\right\}
\end{gathered}
$$

and fixing a trivialization $\Psi$ of $x_{T}^{*} \xi$, define

$$
p=\operatorname{wind}\left(\nu_{P}^{p o s}\right)-\operatorname{wind}\left(\nu_{P}^{n e g}\right) .
$$

One can check that $p \in\{0,1\}$.

Definition 1.16. We define the (generalized) Conley-Zehnder index of the orbit $P$ relative to the unitary trivialization $\Psi$ as

$$
\tilde{\mu}(P, \Psi)=2 \operatorname{wind}\left(\nu_{P}^{n e q}, \Psi\right)+p .
$$

It is proved in [HWZ95b, Theorem 3.10] that for any nondegenerate orbit $P \in \mathcal{P}(\lambda)$,

$$
\tilde{\mu}(P, \Psi)=\mu(P, \Psi),
$$

where $\mu(P, \Psi)$ is the Conley-Zehnder index defined in 1.2 .1 .

\subsection{The action functional}

Let $(M, \lambda)$ be a closed contact manifold of dimension 3 . We define the action functional $\mathcal{A}: C^{\infty}\left(S^{1}=\mathbb{R} / \mathbb{Z}, M\right) \rightarrow \mathbb{R}$ by

$$
\mathcal{A}(\gamma)=\int_{\gamma} \lambda:=\int_{S^{1}} \gamma^{*} \lambda
$$

First variation of the action functional Let $\gamma \in C^{\infty}\left(S^{1}, M\right)$ and let $h \in \Gamma\left(\gamma^{*} T M\right)$ be a vector field along $\gamma$. Consider a function $u:(-\epsilon, \epsilon) \times S^{1} \rightarrow$ $M, \quad(s, t) \mapsto u(s, t)$ satisfying

$$
\left\{\begin{array}{l}
u(0, t)=\gamma(t) \\
\frac{\partial u}{\partial s}(0, t)=h(t)
\end{array}, \forall t \in S^{1}\right.
$$


Then

$$
\begin{aligned}
\left.\frac{d}{d s}\right|_{s=0} \mathcal{A}(u(s, \cdot)) & =\left.\frac{d}{d s}\right|_{s=0} \int_{S^{1}} u(s, \cdot)^{*} \lambda \\
& =\left.\int_{S^{1}} \frac{d}{d s}\right|_{s=0}\left(u^{*} \lambda\right)_{(s, t)}\left(\frac{\partial}{\partial t}\right) d t \\
& =\int_{S^{1}} \mathcal{L}_{\frac{\partial}{\partial s}}\left(u^{*} \lambda\right)_{(0, t)}\left(\frac{\partial}{\partial t}\right) d t \\
& =\int_{S^{1}}\left(i_{\frac{\partial}{\partial s}} d\left(u^{*} \lambda\right)+d i_{\frac{\partial}{\partial s}}\left(u^{*} \lambda\right)\right)_{(0, t)}\left(\frac{\partial}{\partial t}\right) d t \\
& =\int_{S^{1}} d \lambda(h(t), \dot{\gamma}(t)) d t .
\end{aligned}
$$

In the last equality we used the fact that $\int_{S^{1}} d i_{\frac{\partial}{\partial s}}\left(u^{*} \lambda\right)_{(0, t)}\left(\frac{\partial}{\partial t}\right) d t=0$, since the integrand is the differential of a function on $S^{1}$.

It follows that $\gamma$ is a critical point of $\mathcal{A}$, that is, $\left.\frac{d}{d s}\right|_{s=0} \mathcal{A}\left(\gamma_{s}\right)=0$ for all smooth curve $s \mapsto \gamma_{s}$ satisfying $\gamma_{0}=\gamma$, if and only if $\dot{\gamma}(t)$ points in the direction of the Reeb vector field $R(\gamma(t))$ for all $t$. Indeed, assume that $\gamma$ is a critical point of $\mathcal{A}$ and that there exist $Y \in \Gamma\left(\gamma^{*} T M\right)$ and an open set $U$ of $S^{1}$ such that $d \lambda(Y(\gamma(t)), \dot{\gamma}(t))>0$ on $U$. Consider $f \geq 0$ a smooth function with support in $U$ and define the vector field $X=f Y \in \Gamma\left(\gamma^{*} T M\right)$. It follows that $d \lambda(X, \dot{\gamma}) \geq 0$ and $\int_{S^{1}} d \lambda(X(t), \dot{\gamma}(t)) d t>0$ on $U$, a contradiction.

It is clear that for any $T$-periodic solution $x: \mathbb{R} \rightarrow M$ of $\dot{x}(t)=R(x(t))$, the loop $x_{T}: S^{1} \rightarrow M ; x_{T}(t)=x(T t)$ is a critical point of the action functional. On the other hand, every critical point satisfying $\dot{\gamma}(t) \neq 0, \forall t \in$ $S^{1}$ and $\mathcal{A}(\gamma)=T$ is the reparametrization of a $T$-periodic Reeb orbit. Indeed, assume $\gamma$ is a critical point satisfying $\dot{\gamma}(t) \neq 0, \forall t \in S^{1}$, i.e, $\dot{\gamma}(t)=f(t) R(t)$ and $f(t) \neq 0$. Let $T=\mathcal{A}(\gamma)$. Then there exists a reparametrization $\sigma(t)=$ $\gamma(g(t))$ such that $\dot{\sigma}(t)=T R(\sigma(t))$. Indeed, consider $f: \mathbb{R} \rightarrow \mathbb{R}$ 1-periodic and $h(t)=\int_{0}^{t} f(\tau) d \tau$. Since $h(t+1)=T+\int_{0}^{t} f(\tau) d \tau, \frac{1}{T} h$ can be seen as a degree one map $S^{1} \rightarrow S^{1}$. Since $f(t) \neq 0 \forall t$, then $\frac{1}{T} h$ is a diffeomorphism (bijective immersion). Define $g(t)=\left(\frac{1}{T} h\right)^{-1}(t)$ and $\sigma(t)=\gamma(g(t))$. Then $\dot{\sigma}(t)=T R(\sigma(t))$.

The asymptotic operator as the "hessian of $\mathcal{A}_{\lambda}$ in the direction $\xi$ " Let $\gamma(t)=x(T t)$ and let $X, Y \in \Gamma\left(\gamma^{*} \xi\right)$ be vector fields along $\gamma$ in the contact direction. Let $u: U \times S^{1} \rightarrow M,(r, s, t) \mapsto u(r, s, t)$ be a smooth function 
such that

$$
u(0,0, t)=\gamma(t), \frac{\partial u}{\partial r}(0,0, t)=X(t) \text { and } \frac{\partial u}{\partial s}(0,0, t)=Y(t)
$$

where $U \ni 0$ is an open set in $\mathbb{R}^{2}$. Define $\tilde{u}: U \rightarrow \mathbb{C}^{\infty}\left(S^{1}, M\right)$ by $\tilde{u}(r, s)=$ $u(r, s, \cdot)$. It follows that

$$
\frac{\partial}{\partial r}(\mathcal{A} \circ \tilde{u})(s, r)=\int_{S^{1}} d \lambda_{u(r, s, t)}\left(\frac{\partial u}{\partial s}(r, s, t), \frac{\partial u}{\partial t}(r, s, t)\right) d t .
$$

Let $J$ be a $d \lambda$-compatible smooth complex structure on $\xi$ and let $g$ be the Riemannian metric on $M$ defined by $g(u, v)=\lambda(u) \lambda(v)+d \lambda\left(\pi_{\xi} u, J \pi_{\xi} v\right)$. Let $\nabla$ be the Levi-Civitta connection associated with $g$. Then

$$
\begin{aligned}
\left.\frac{\partial^{2}}{\partial s \partial r}(\mathcal{A} \circ \tilde{u})\right|_{(0,0)} & =\left.\frac{\partial}{\partial r}\right|_{(0,0)} \int_{S^{1}} d \lambda_{u(r, s, t)}\left(\frac{\partial u}{\partial s}(r, s, t), \frac{\partial u}{\partial t}(r, s, t)\right) d t \\
= & \left.\partial_{r}\right|_{(0,0)} \int_{S^{1}}-d \lambda\left(J \pi_{\xi} \partial_{t} u, J \pi_{\xi} \partial_{s} u\right) d t \\
= & \left.\int_{S^{1}} \partial_{r}\right|_{(0,0)} g\left(-J \pi_{\xi} \partial_{t} u, \pi_{\xi} \partial_{s} u\right) d t \\
= & \int_{S^{1}} g\left(\nabla_{X(t)}\left(-\left.J \pi_{\xi} \partial_{t} u(r, 0, t)\right|_{r=0}, Y(t)\right)\right. \\
& +g \underbrace{-J \pi_{\xi} \dot{\gamma}(t)}_{=0},\left.\nabla_{X(t)} \pi_{\xi} \partial_{s} u\right|_{(0,0, t)}) d t .
\end{aligned}
$$

In the last equality we have used $\nabla_{X} g=0$. For the first term in the integral above, we compute

$$
\begin{aligned}
\left.\nabla_{X}\left(-J \pi_{\xi} \partial_{t} u(r, 0, t)\right)\right|_{r=0}= & -\left.J \nabla_{X}\left(\partial_{t} u(r, 0, t)-\lambda\left(\partial_{t} u(r, 0, t)\right) R(u(r, 0, t))\right)\right|_{r=0} \\
= & -J\left\{\left.\nabla_{X} \partial_{t} u(r, 0, t)\right|_{r=0}-\left.\nabla_{X} \lambda\left(\partial_{t} u(r, 0, t)\right)\right|_{r=0} R(\gamma(t))\right. \\
& \left.-\left.\lambda(\dot{\gamma}(t)) \nabla_{X} R\right|_{\gamma(t)}\right\}
\end{aligned}
$$

where in the first equality, we have used $\pi_{\xi} \partial_{t} u(0,0, t)=0$. 
Remark 1.17. $\left.\nabla_{X} \lambda\left(\partial_{t} u(r, 0, t)\right)\right|_{r=0}=0$. Ideed,

$$
\begin{aligned}
\left.\nabla_{X} \lambda\left(\partial_{t} u(r, 0, t)\right)\right|_{r=0} & =\left.\left(u^{*} \nabla\right)_{\frac{\partial}{\partial r}} u^{*} \lambda\left(\frac{\partial}{\partial t}\right)\right|_{(0,0, t)} \\
& =\left.\frac{\partial}{\partial r} \cdot u^{*} \lambda\left(\frac{\partial}{\partial t}\right)\right|_{(0,0, t)} \\
= & \left.d u^{*} \lambda\left(\frac{\partial}{\partial r}, \frac{\partial}{\partial t}\right)\right|_{(0,0, t)}+\left.\frac{\partial}{\partial t} \cdot u^{*} \lambda\left(\frac{\partial}{\partial r}\right)\right|_{(0,0, t)} \\
& -u^{*} \lambda\left(\left[\frac{\partial}{\partial r}, \frac{\partial}{\partial t}\right]\right) \\
= & 0 .
\end{aligned}
$$

In the last equality we have used $d \lambda(X(t), \dot{\gamma}(t))=0$ and $\lambda\left(\partial_{r} u\right)=0$ along $t \mapsto(0,0, t) \cdot{ }^{9}$

Remark 1.18. $\nabla_{X(t)} \partial_{t} u(\gamma(t))=\nabla_{\dot{\gamma}(t)} X(\gamma(t))$. Indeed, consider local coordinates $\left(x_{1}, \ldots, x_{2 n+1}\right)$ on $M$ near $\gamma(t)$ and write

$$
\begin{gathered}
\partial_{t} u(r, 0, t)=\sum_{k} \partial_{t} u_{k}(r, 0, t) \frac{\partial}{\partial x_{k}}(u(r, 0, t)), \\
X(t)=\sum_{i} \partial_{r} u_{i}(0,0, t) \frac{\partial}{\partial x_{i}}(\gamma(t)), \quad \dot{\gamma}(t)=\sum_{l} \dot{\gamma}_{l}(t) \frac{\partial}{\partial x_{l}}(\gamma(t)) .
\end{gathered}
$$

Thus,

$$
\begin{gathered}
\nabla_{X(t)} \partial_{t} u(\gamma(t))=\sum_{k} \partial_{r} \partial_{t} u_{k}(0,0, t) \frac{\partial}{\partial x_{k}}(\gamma(t))+\sum_{i, k} \dot{\gamma}_{k}(t) \partial_{r} u_{i}(0,0, t) \nabla_{\frac{\partial}{\partial x_{i}}} \frac{\partial}{\partial x_{k}}, \\
\nabla_{\dot{\gamma}} X(\gamma(t))=\sum_{i} \partial_{t} \partial_{r} u_{i}(0,0, t) \frac{\partial}{\partial x_{i}}(\gamma(t))+\sum_{i, l} \partial_{r} u_{i}(0,0, t) \dot{\gamma}_{l}(t) \nabla_{\frac{\partial}{\partial x_{l}}} \frac{\partial}{\partial x_{i}}
\end{gathered}
$$

The equality follows from $\nabla$ being symmetric.

It follows from the remarks above that

$$
\left.\nabla_{X}\left(-J \pi_{\xi} \partial_{t} u(r, 0, t)\right)\right|_{r=0}=-J\left(\nabla_{\dot{\gamma}} X-T \nabla_{X} R\right)(\gamma(t)) .
$$

\footnotetext{
${ }^{9}$ Also, we have used the formula $d \omega(X, Y)=X \cdot \omega(Y)-Y \cdot \omega(X)-\omega([X, Y])$.
} 
24 CHAPTER 1. CONTACT GEOMETRY AND REEB DYNAMICS

We conclude that

$$
\begin{aligned}
\left.\frac{\partial^{2}}{\partial s \partial r}(\mathcal{A} \circ \tilde{u})\right|_{(0,0)} & =\int_{S^{1}} d \lambda\left(A_{P, J}(X), J Y\right) d t \\
& =T \int_{S^{1}} d \lambda\left(Y(t), \mathcal{L}_{R} X(t)\right) d t
\end{aligned}
$$

where $A_{P, J}$ is the asymptotic operator defined in 1.8 . 


\section{Chapter 2}

\section{Finite energy surfaces in symplectizations}

\subsection{Almost complex structures in symplectiza- tions}

Let $W$ is a smooth manifold. An almost complex structure on $W$ is a complex structure on the tangent bundle $T W$. If $J$ is an almost complex structure on $W$, the pair $(W, J)$ is called an almost complex manifold.

Definition 2.1. Let $(W, J)$ be an almost complex manifold and let $(\Sigma, j)$ be a Riemann surface. A smooth map $u: \Sigma \rightarrow W$ is called a $J$-holomorphic curve if the differential $d u$ is complex-linear, that is, satisfies the equation

$$
J(u) \circ d u=d u \circ j .
$$

Let $(W, \omega)$ be a symplectic manifold. An $\omega$-compatible almost complex structure on $(W, \omega)$ is an $\omega$-compatible complex structure on $T W$ (see definition 1.10). We denote the set of $\omega$-compatible almost complex structures on $(W, \omega)$ by $\mathcal{J}(W, \omega)$.

Let $(M, \lambda)$ be a closed contact 3-manifold and let $\xi=\operatorname{ker} d \lambda$ be the associated contact structure. Recall that $\left(\xi,\left.d \lambda\right|_{\xi}\right)$ is a symplectic vector bundle. Fixed a $d \lambda$-compatible complex structure $J \in \mathcal{J}(\xi, d \lambda)$, there is a canonical extension $\tilde{J}$ of the complex structure $J$ to $T(\mathbb{R} \times M)$ defined by

$$
\tilde{J} \partial_{a}=R_{\lambda},\left.\quad \tilde{J}\right|_{\xi}=J .
$$

Here $a$ is the coordinate on $\mathbb{R}$ and $R_{\lambda}$ is the Reeb vector field associated with 
$\lambda$. These equations uniquely define the extension $\tilde{J}$ because of the splitting

$$
T(\mathbb{R} \times M)=\mathbb{R} \partial_{a} \oplus \mathbb{R} R_{\lambda} \oplus \xi .
$$

Note that for all $(h, k) \in T(\mathbb{R} \times M)$,

$$
\tilde{J}(h, k)=\left(-\lambda(k), J \pi_{\xi} k+h R_{\lambda}\right) .
$$

Proposition 2.2. The almost complex structure $\tilde{J}$ defined by (2.1) is $d \lambda_{\phi^{-}}$ compatible, where $\lambda_{\phi}$ is the 1-form defined by 1.2 , for any $\phi \in C^{\infty}(\mathbb{R},(0,+\infty))$ satisfying $\phi^{\prime}>0$.

Proof. Let $(a, m) \in \mathbb{R} \times M$ and $(h, k),\left(h^{\prime}, k^{\prime}\right) \in T_{(a, m)}(\mathbb{R} \times M)$. First note that

$$
d \lambda_{\phi}(a, m)=d \phi_{(a, m)} \wedge \lambda_{m}+\phi(a) d \lambda_{m},
$$

where $\phi$ is seen as a function $(a, m) \mapsto \phi(a)$. Then

$$
\begin{aligned}
d \lambda_{\phi}\left(\tilde{J}(h, k), \tilde{J}\left(h^{\prime}, k^{\prime}\right)\right) & =d \lambda_{\phi}\left(\left(-\lambda(k), J \pi k+h R_{\lambda}\right),\left(-\lambda\left(k^{\prime}\right), J \pi k^{\prime}+h^{\prime} R_{\lambda}\right)\right) \\
& =\phi^{\prime}(a)(-\lambda(k)) h^{\prime}-\phi^{\prime}(a)\left(-\lambda\left(k^{\prime}\right)\right) h+\phi(a) d \lambda_{m}\left(J \pi k, J \pi k^{\prime}\right) \\
& =d \phi_{a} \wedge \lambda\left((h, k),\left(h^{\prime}, k^{\prime}\right)\right)+\phi(a) d \lambda\left(k, k^{\prime}\right) \\
& =d \lambda_{\phi}\left((h, k),\left(h^{\prime}, k^{\prime}\right)\right)
\end{aligned}
$$

proving that $\tilde{J}$ is a linear symplectomorphism. Also

$$
\begin{aligned}
d \lambda_{\phi}((h, k), \tilde{J}(h, k)) & =d \lambda_{\phi}\left((h, k),\left(-\lambda(k), J \pi k+h R_{\lambda}\right)\right) \\
& =\phi^{\prime}(a) h h-\phi^{\prime}(a)(-\lambda(k)) \lambda(k)+\phi(a) d \lambda(k, J \pi k) \\
& =\phi^{\prime}(a)\left(h^{2}+\lambda(k)^{2}\right)+\phi(a) d \lambda(\pi k, J \pi k),
\end{aligned}
$$

so that $d \lambda_{\phi}((h, k), \tilde{J}(h, k))>0$, if $(h, k) \neq 0$.

\section{$2.2 \quad$ Finite energy surfaces}

Let $(M, \lambda)$ be a closed contact manifold of dimension 3. Fixing an almost complex structure $J \in \mathcal{J}(\xi, d \lambda)$, we are interested in $\tilde{J}$-holomorphic curves

$$
\tilde{u}:(a, u):(\dot{S}, j) \rightarrow(\mathbb{R} \times M, \tilde{J})
$$

where $\dot{S}=S \backslash \Gamma,(S, j)$ is a closed Riemann surface and $\Gamma$ is a finite set of points in $S$.

Define

$$
\left.\Sigma=\left\{\phi \in C^{\infty}(\mathbb{R},[0,1]) \mid \phi^{\prime} \geq 0\right)\right\}
$$


and for $\phi \in \Sigma$, let $\lambda_{\phi}$ be the 1 -form on $\mathbb{R} \times M$ defined by

$$
\lambda_{\phi}(a, x)(h, k)=\phi(a) \lambda(m)(k) .
$$

If $\tilde{u}=(a, u)$ is a $\tilde{J}$-holomorphic curve, we can compute in local holomorphic coordinates $s+i t \in \mathbb{C}$

$$
\begin{aligned}
\tilde{u}^{*} d \lambda_{\phi}(\partial s, \partial t) & =d \lambda_{\phi}\left(\tilde{u}_{s}, \tilde{u}_{t}\right) \\
& =\frac{1}{2}\left(d \lambda_{\phi}\left(\tilde{u}_{s}, \tilde{J} \tilde{u}_{s}\right)+d \lambda_{\phi}\left(\tilde{u}_{t}, \tilde{J} \tilde{u}_{t}\right)\right) \\
& =\frac{1}{2}\left(\phi^{\prime}(a)\left(a_{s}^{2}+a_{t}^{2}+\lambda\left(u_{s}\right)^{2}+\lambda\left(u_{t}\right)^{2}\right)+\phi(a)\left(\left|\pi u_{s}\right|_{J}^{2}+\left|\pi u_{t}\right|_{J}^{2}\right)\right) \\
& \geq 0
\end{aligned}
$$

where $|\cdot|_{J}^{2}=d \lambda(\cdot, J \cdot)$. Therefore, if $\tilde{u}$ is $\tilde{J}$-holomorphic, then

$$
0 \leq \int_{\dot{S}} \tilde{u}^{*} d \lambda_{\phi} \leq \infty
$$

for any $\phi \in \Sigma$, since (2.3) is non-negative. Also, $E(\tilde{u})=0$ if and only if $\tilde{u}$ is constant.

Definition 2.3 (Energy). We define the energy of a $\tilde{J}$-holomorphic curve $\tilde{u}: \dot{S} \rightarrow \mathbb{R} \times M$ by

$$
E(\tilde{u})=\sup _{\phi \in \Sigma} \int_{S \backslash \Gamma} \tilde{u}^{*} d(\phi \lambda),
$$

where $\Sigma$ is defined by $(2.2)$.

Definition 2.4 (Finite energy surface). Let $(S, j)$ be a closed Riemann surface and let $\Gamma \subset S$ be a finite set. A smooth map $\tilde{u}: S \backslash \Gamma \rightarrow \mathbb{R} \times M$ is called a finite energy surface if it is $\tilde{J}$-holomorphic and satisfies the energy condition

$$
0<E(\tilde{u})<+\infty .
$$

The elements of $\Gamma$ are called punctures.

Write $\tilde{u}=(a, u)$. Let $z \in \Gamma$ be a puncture and take a holomorphic chart $\varphi:(U, 0) \rightarrow(\varphi(U), z)$ centered at $z$. We call $(s, t) \simeq \varphi\left(e^{-2 \pi(s+i t)}\right)$ positive exponential coordinates and $(s, t) \simeq \varphi\left(e^{2 \pi(s+i t)}\right)$ negative exponential coordinates around $z$.

Set $\tilde{u}(s, t)=\tilde{u} \circ \varphi\left(e^{-2 \pi(s+i t)}\right)$, for $s>>1$. Using (2.3), Stokes Theorem 
and the energy condition 2.4 , one can prove that the limit

$$
m(z)=\lim _{s \rightarrow+\infty} \int_{\{s\} \times S^{1}} u^{*} \lambda
$$

exists.

The puncture $z$ is called removable if $m=0$, positive if $m>0$ and negative if $m<0$. By an application of Gromov's removable singularity theorem Gro85, one can prove that $\tilde{u}$ can be smoothly extended to a removable puncture. Thus, in the following we assume that all punctures are positive or negative and use the notation $\Gamma=\Gamma^{+} \cup \Gamma^{-}$to distinguish positive and negative punctures.

If $\tilde{u}: S \backslash \Gamma \rightarrow \mathbb{R} \times M$ is a finite energy surface, then $\Gamma \neq \emptyset$. Indeed, suppose $\Gamma=\emptyset$. Since $\partial S=\emptyset$, we conclude by Stokes Theorem that $\int_{S} \tilde{u}^{*} d \lambda_{\phi}=0$ for any $\phi \in \Sigma$, which contradicts $E(\tilde{u})>0$. Again using Stokes Theorem and (2.3), one can show that $\Gamma^{+} \neq \emptyset$.

Definition 2.5 ( $d \lambda$-area). The $d \lambda$-area of a $\tilde{J}$-holomorphic curve is given by the formula

$$
A(\tilde{u})=\int_{\dot{S}} \tilde{u}^{*} d \lambda=\int_{\dot{S}} u^{*} d \lambda .
$$

By (2.3), $A(\tilde{u}) \geq 0$ and $A(\tilde{u})=0$ if and only if $\pi \cdot d u \equiv 0$.

Theorem 2.6. [HWZ95b, Theorem 6.11] Let $\tilde{u}=(a, u): \mathbb{C} \backslash \Gamma \rightarrow \mathbb{R} \times$ $M$ be a finite-energy punctured sphere, where $\Gamma \subset \mathbb{C}$ is the finite set of negative punctures and $\infty$ is the unique positive puncture. If $\pi \cdot d u \equiv 0$, where $\pi: T S^{3}=\mathbb{R} R_{\lambda} \oplus \xi \rightarrow \xi$ is the projection, then there exists a nonconstant polynomial $p: \mathbb{C} \rightarrow \mathbb{C}$ and a periodic Reeb orbit $P=(x, T) \in \mathcal{P}(\lambda)$ such that

$$
p^{-1}(0)=\Gamma \text { and } \tilde{u}=F_{P} \circ p
$$

where $F_{P}: \mathbb{C} \backslash\{0\} \rightarrow \mathbb{R} \times M$ is defined by $F_{P}\left(z=e^{2 \pi(s+i t)}\right)=(T s, x(T t))$.

Remark 2.7. Note that in Theorem 2.6, if $p$ has degree $k$, then the asymptotic limit of $\tilde{u}$ at $\infty$ is $P^{k}=(x, k T)$.

Corollary 2.8. If $\tilde{u}=(a, u): \mathbb{C} \rightarrow \mathbb{R} \times M$ is a finite-energy plane, then $\int_{\mathbb{C}} u^{*} d \lambda>0$.

\subsubsection{Asymptotic behavior}

The existence of a finite energy surface in the symplectization $\mathbb{R} \times M$ is equivalent to the existence of closed Reeb orbits in $(M, \lambda)$. This is a consequence of the following theorem proved in [Hof93] and [HWZ96]. 
Theorem 2.9. Let $\tilde{u}=(a, u): S \backslash \Gamma \rightarrow \mathbb{R} \times M$ be a finite energy surface. Assume $z$ is non removable and let $\epsilon= \pm 1$ be the sign of $m(z)$, defined in (2.5). Fix a sequence $s_{n} \rightarrow+\infty$. Then there exists a non constant trajectory of the Reeb flow $x: \mathbb{R} \rightarrow M$ with period $T>0$ and a subsequence $s_{n_{k}}$ such that

$$
\lim _{k \rightarrow+\infty}\left\{t \mapsto u\left(s_{n_{k}}, t\right)\right\}=\{t \mapsto x(\epsilon T t)\}
$$

in the $C^{\infty}\left(S^{1}, M\right)$ topology. If the orbit $(x, T)$ is nondegenerate, then

$$
\lim _{s \rightarrow+\infty}\{t \mapsto u(s, t)\}=\{t \mapsto x(\epsilon T t)\}
$$

In the nondegenerate case, there is a unique periodic orbit $(x, T)$ associated with the puncture $z$. It has period $T=|m|$ and is called the asymptotic limit of $\tilde{u}$ at $z$.

Definition 2.10. A Martinet's tube for a simply covered orbit $P=(x, T) \in$ $\mathcal{P}(\lambda)$ is a pair $(U, \psi)$, where $U$ is a neighborhood of $x(\mathbb{R})$ in $M$ and $\psi: U \rightarrow$ $S^{1} \times B$ is a diffeomorphism (here $B \subset \mathbb{R}^{2}$ is an open ball centered at the origin) satisfying

- There exists $f: S^{1} \times B \rightarrow \mathbb{R}^{+}$such that $\left.f\right|_{S^{1} \times\{0\}} \equiv T,\left.d f\right|_{S^{1} \times\{0\}} \equiv 0$ and $\psi^{*}\left(f\left(d \theta+x_{1} d x_{2}\right)\right)=\lambda$, where $\theta$ is the coordinate on $S^{1}$ and $\left(x_{1}, x_{2}\right)$ are coordinates on $\mathbb{R}^{2}$;

- $\psi\left(x_{T}(t)\right)=(t, 0,0)$.

The coordinates $\left(\theta, x_{1}, x_{2}\right)$ are referred to as Martinet's coordinates.

The existence of such Martinet's tubes is proved in [HWZ96] for any simply covered orbit $P \in \mathcal{P}(\lambda)$.

From now on we assume that $\lambda$ is nondegenerate.

A more precise description of the asymptotic behavior of a finite energy surface is given by the following theorem of [HWZ96].

Theorem 2.11 ([HWZ96]). Let $\lambda$ be a nondegenerate contact form on $M$. Let $z_{0} \in \Gamma$ be a positive puncture of the finite energy $\tilde{J}$-holomorphic sphere $\tilde{u}=(a, u): S^{2} \backslash \Gamma \rightarrow \mathbb{R} \times M$ and let $(s, t)$ be positive exponential coordinates near $z_{0}$. Let $P=(x, T)$ be the asymptotic limit of $\tilde{u}$ at $z_{0}$ and let $k$ be a positive integer such that $T=k T_{\text {min }}$, where $T_{\text {min }}$ is the least positive period of $x$. Let $\left(\theta, x_{1}, x_{2}\right)$ be Martinet's coordinates in a neighborhood $U \subset M$ of $P_{\text {min }}=\left(x, T_{\text {min }}\right)$ given by a Martinet's tube $(U, \psi)$. Then the map $\psi \circ u(s, t)=\left(\theta(s, t), x_{1}(s, t), x_{2}(s, t)\right)$ is defined for all large $s$ and either 
$\left(x_{1}(s, t), x_{2}(s, t)\right) \equiv 0$ or there are constants $s_{0}, A_{i j}, r_{0} \in \mathbb{R}^{+}, a_{0} \in \mathbb{R}$, a function $R: \mathbb{R} \times S^{1} \rightarrow \mathbb{R}^{2}$ and an eigensection $v(t)$ of the asymptotic operator $A_{P, J}(1.8)$, associated with a negative eigenvalue $\alpha \in \sigma\left(A_{P, J}\right)$, such that

$$
\begin{array}{r}
\left|\partial_{s}^{i} \partial_{t}^{j}\left(a(s, t)-\left(T s+a_{0}\right)\right)\right| \leq A_{i j} e^{-r_{0} s} \\
\left|\partial_{s}^{i} \partial_{t}^{j}(\theta(s, t)-k t)\right| \leq A_{i j} e^{-r_{0} s} \\
\left(x_{1}(s, t), x_{2}(s, t)\right)=e^{\int_{s_{0}}^{s} \alpha(r) d r}(e(t)+R(s, t)) \\
\left|\partial_{s}^{i} \partial_{t}^{j} R(s, t)\right|,\left|\partial_{s}^{i} \partial_{t}^{j}(\alpha(s)-\alpha)\right| \leq A_{i j} e^{-r_{0} s}
\end{array}
$$

for all large $s$ and $i, j \in \mathbb{N}$. Here $\theta(s, t)$ is seen as a map on the universal cover $\mathbb{R}$ of $S^{1}$, e : $S^{1} \rightarrow \mathbb{R}^{2}$ represents the eigensection $v(t)$ in the coordinates induced by $\psi$, and $\alpha:\left[s_{0}, \infty\right) \rightarrow \mathbb{R}$ is a smooth function such that $\alpha(s) \rightarrow$ $\alpha$, as $s \rightarrow \infty$.

A similar statement holds if $z_{0}$ is a negative puncture. In this case, we use negative exponential coordinates near $z_{0}, e^{-r_{0} s}$ is replaced by $e^{r_{0} s}$ and the eigenvalue $\alpha$ of $A_{P, J}$ is positive.

The eigenvalue $\alpha$ and the eigensection $v(t)$, as in Theorem 2.11, will be refered to as the asymptotic eigenvalue and asymptotic eigensection of $\tilde{u}$ at the puncture $z_{0}$.

\subsubsection{Somewhere injective curves}

Definition 2.12. A $\tilde{J}$-holomorphic curve $\tilde{u}: S \backslash \Gamma \rightarrow \mathbb{R} \times M$ is called somewhere injective if there exists a point $z_{0} \in S \backslash \Gamma$ satisfying

$$
\tilde{u}^{-1}\left(\tilde{u}\left(z_{0}\right)\right)=\left\{z_{0}\right\} \text { and } d \tilde{u}\left(z_{0}\right) \neq 0 .
$$

Every finite energy curve with nondegenerate asymptotic limits factors through a somewhere injective one. This is the content of Theorem 2.13 and Corollary 2.15 below, that are generalizations of the corresponding result about finite energy planes proved in Theorem 6.2 of [HWZ95b].

Theorem 2.13. Let $\tilde{u}:(S \backslash \Gamma, j) \rightarrow(\mathbb{R} \times M, \tilde{J})$ be a nonconstant finite energy $\tilde{J}$-holomorphic curve asymptotic to nondegenerate Reeb orbits, where $(S, j)$ is a closed Riemann surface and $\Gamma \subset S$ is a finite set. Then there exists a factorization

$$
\tilde{u}=\tilde{v} \circ \varphi
$$

where

- $\tilde{v}:\left(S^{\prime} \backslash \Gamma^{\prime}, j^{\prime}\right) \rightarrow(\mathbb{R} \times M, \tilde{J})$ is a finite energy $\tilde{J}$-holomorphic curve that is embedded outside a finite set of critical points and self-intersections. 
- $\varphi:(S, j) \rightarrow\left(S^{\prime}, j^{\prime}\right)$ is a holomorphic map of positive degree.

A proof of theorem 2.13 can be found in [Nel15].

One of the ingredients of the proof, that will be useful to us later, is the lemma below, that follows from results in Sie08.

Lemma 2.14. Assume $u:(S \backslash \Gamma, j) \rightarrow(\mathbb{R} \times M, \tilde{J})$ is a finite energy curve and $z_{0} \in \Gamma$ is a puncture whose asymptotic limit $P=(x, T)$ is nondegenerate. Then a punctured neighborhood $\dot{U}$ of $z_{0}$ in $S \backslash \Gamma$ can be biholomorphically identified with the punctured unit disk $\dot{\mathbb{D}}=\mathbb{D} \backslash\{0\} \subset \mathbb{C}$ and

$$
u(z)=v\left(z^{k}\right), \forall z \in \dot{\mathbb{D}},
$$

where $k \in \mathbb{Z}^{+}$divides the multiplicity of the orbit $P$ and $v:(\dot{\mathbb{D}}, i) \rightarrow(\mathbb{R} \times$ $M, \tilde{J})$ is an embedded finite energy map. If $w:\left(S^{\prime} \backslash \Gamma^{\prime}, j^{\prime}\right) \rightarrow(\mathbb{R} \times M, \tilde{J})$ is another finite energy curve with puncture $z_{0}^{\prime} \in S^{\prime}$ then the images of $u$ near $z_{0}$ and $w$ near $z_{0}^{\prime}$ are either identical or disjoint.

In particular, if $\tilde{u}: \mathbb{C} \backslash \Gamma \rightarrow \mathbb{R} \times M$ is a finite energy sphere with a unique positive puncture at $\infty$, then we have the following corollary, which will be useful later.

Corollary 2.15. Let $\tilde{u}: \mathbb{C} \backslash \Gamma \rightarrow \mathbb{R} \times M$ be a finite energy sphere asymptotic to nondegenerate Reeb orbits and with a unique positive puncture at $\infty$. Then there exists a somewhere injective finite energy sphere $\tilde{v}: \mathbb{C} \backslash \Gamma^{\prime} \rightarrow \mathbb{R} \times M$ and a polynomial $p: \mathbb{C} \rightarrow \mathbb{C}$ mapping $\Gamma$ to $\Gamma^{\prime}$ such that

$$
\tilde{u}=\tilde{v} \circ p .
$$

Proof. Using Theorem 2.13, the proof follows the same arguments found in the end of the proof of [HWZ95b, Theorem 6.2]. By Theorem 2.13, $\tilde{u}$ factors as $\tilde{v} \circ \varphi$, for a finite energy curve $\tilde{v}:\left(S^{\prime}, j^{\prime}\right) \rightarrow \mathbb{R} \times M$ and a holomorphic $\operatorname{map} \varphi:\left(S^{2}, i\right) \rightarrow\left(S^{\prime}, j^{\prime}\right)$ with $\operatorname{deg} \varphi>0$.

First we show that we can assume $\left(S^{\prime}, j^{\prime}\right)=\left(S^{2}, i\right)$. Using Poincaré duality, the formula $\varphi_{*}(\cdot) \cap \cdot=\varphi_{*}\left(\cdot \cap \varphi^{*}(\cdot)\right)$ for the cap product and $\operatorname{deg} \varphi>0$, we conclude that $\varphi^{*}: H^{1}\left(S^{\prime}\right) \rightarrow H^{1}\left(S^{2}\right)$ is injective, and consequently that $H_{1}\left(S^{\prime}\right)=0$. This implies that $S^{\prime}$ is the topological 2-sphere. By the Uniformization theorem, $\left(S^{\prime}, j^{\prime}\right)$ is biholomorphic to the Riemann sphere $\left(S^{2}, i\right)$. Thus, we can assume $\tilde{u}=\tilde{v} \circ \varphi$, where $\varphi:\left(S^{2}, i\right) \rightarrow\left(S^{2}, i\right)$ is a holomorphic map with positive degree.

After a reparametrization, we can assume $\infty$ is a positive puncture of $\tilde{v}$. Note that $\tilde{v}$ must have only one positive puncture, $\varphi(\infty)=\infty$ and $\varphi(\mathbb{C})=\mathbb{C}$. This follows from the surjectivity of $\varphi$ and the fact that $\tilde{u}$ has 
a unique positive puncture at $\infty$. The conclusion of the proof follows form the fact that any homolomorphic map $S^{2} \rightarrow S^{2}$ mapping $\mathbb{C}$ to itself is a extension of a polynomial to the Riemman sphere.

\subsubsection{Algebraic invariants}

Let $\lambda$ be a nondegenerate contact form on $M$, fix $J \in \mathcal{J}(\xi, d \lambda)$ and let $\tilde{u}: S \backslash \Gamma \rightarrow \mathbb{R} \times M$ be a $\tilde{J}$-holomorphic finite energy surface.

Let $\pi: T M=\mathbb{R} R_{\lambda} \oplus \xi \rightarrow \xi$ be the projection along the Reeb vector field and assume that $\pi \cdot d u$ is not identically zero. In HWZ95b, it is proved that the set where $\pi \cdot d u$ vanishes is finite, and it is defined a local degree associated to each zero of $\pi \cdot d u$, that is always positive. The integer

$$
\operatorname{wind}_{\pi}(\tilde{u}) \geq 0
$$

is defined as the sum of such local degrees over all zeros of $\pi \cdot d u$.

The bundle $\left(u^{*} \xi, d \lambda, J\right) \rightarrow \dot{S}$ has a unitary trivialization. Indeed, since $\Gamma \neq \emptyset, S \backslash \Gamma$ has the homotopy type of a wedge of circles. This implies that the first Chern class of the complex bundle $\left(u^{*} \xi, J\right)$ is zero and, thus, $\left(u^{*} \xi, J\right)$ is trivial.

Consider a unitary trivialization $\Psi:\left(u^{*} \xi, d \lambda, J\right) \rightarrow \dot{S} \times \mathbb{R}^{2}$. For $z \in \Gamma$ fix positive cylindrical coordinates $(s, t)$ at $z$ and define

$$
\operatorname{wind}_{\infty}(\tilde{u}, z, \Psi)=\lim _{s \rightarrow+\infty} \operatorname{wind}\left(t \mapsto \pi \cdot \partial_{s} u\left(s, \epsilon_{z} t\right),\left.\Psi\right|_{u\left(s, \epsilon_{z} \cdot\right)^{*} \xi}\right) \in \mathbb{Z}
$$

where $\epsilon_{z}$ is the sign of the puncture $z$. The winding number on the right is defined as in (1.12), and is independent of the choice of $J$ and of the holomorphic chart. This limit is well defined since $\pi \cdot \partial_{s} u(s, t)$ does not vanish for $s$ sufficiently large. The asymptotic winding number of $\tilde{u}$ is defined by

$$
\operatorname{wind}_{\infty}(\tilde{u})=\sum_{z \in \Gamma^{+}} \operatorname{wind}_{\infty}(\tilde{u}, z, \Psi)-\sum_{z \in \Gamma^{-}} \operatorname{wind}_{\infty}(\tilde{u}, z, \Psi)
$$

It is proved in HWZ95b that this sum does not depend on the chosen trivialization $\Psi$.

Remark 2.16. Later on we will only deal with the tight contact structure $\xi_{0}$ on $S^{3}$, which is a trivial symplectic bundle. Consider a global symplectic

\footnotetext{
${ }^{1}\left(u^{*} \xi, J\right)$ trivial complex bundle $\Rightarrow\left(u^{*} \xi, d \lambda, J\right) \rightarrow \dot{S}$ has a unitary trivialization. See MS17, sections 2.6 and 2.7 for more details.
} 
trivialization

$$
\Psi: \xi_{0} \rightarrow S^{3} \times \mathbb{R}^{2}
$$

Then $\operatorname{wind}_{\infty}(\tilde{u}, z, \Psi)$ is the winding number of the asymptotic eigensection given by Theorem 2.11, with respect to the trivialization $\Psi$. Also, by Lemma 1.7. $\operatorname{wind}_{\infty}(\tilde{u}, z, \Psi)$ does not depend on the chosen global symplectic trivialization.

Theorem 2.17. [HWZ95b, Theorem 5.6] Let $\tilde{u}=(a, u)$ be a finite energy surface defined on $S \backslash \Gamma$, where $(S, j)$ is a closed Riemann surface and $\Gamma \subset S$ is a finite set consisting of nonremovable punctures. Assume that $\pi \cdot d u$ is not identically zero. Then

$$
\operatorname{wind}_{\pi}(\tilde{u})=\operatorname{wind}_{\infty}(\tilde{u})-\chi(S)+\# \Gamma,
$$

where $\chi(S)$ is the Euler characteristic of $S$.

Definition 2.18. A finite energy plane $\tilde{u}$ is called fast if its asymptotic limit at $\infty$ is a simply covered Reeb orbit and $\operatorname{wind}_{\pi}(\tilde{u})=0$.

\subsubsection{Fredholm theory}

Fix $J \in \mathcal{J}(\xi, d \lambda)$ and let $\tilde{u}: S \backslash \Gamma \rightarrow \mathbb{R} \times M$ be a $\tilde{J}$-holomorphic finite energy surface.

Let $\Psi:\left(u^{*} \xi, d \lambda\right) \rightarrow \dot{S} \times \mathbb{R}^{2}$ be a symplectic trivialization. Fix a puncture $z \in \Gamma$ and let $P=(x, T)$ be the asymptotic limit of $\tilde{u}$ at $z$. The trivialization $\Psi$ induces a homotopy class of oriented trivializations $\left[\Psi_{z}\right]$ of $x_{T}^{*} \xi$. Define $\mu^{\Psi}(z):=\mu\left(P, \Psi_{z}\right)$, where $\mu\left(P, \Psi_{z}\right)$ is the Conley-Zehnder index of the asymptotic limit $P$ of $\tilde{u}$ at $z$, with respect to the trivialization $\Psi_{z}$.

Definition 2.19. We define the Conley-Zehnder index of $\tilde{u}$ by

$$
\mu(\tilde{u})=\sum_{z \in \Gamma^{+}} \mu^{\Psi}(z)-\sum_{z \in \Gamma^{-}} \mu^{\Psi}(z) .
$$

It is proved in HWZ95b that this sum does not depend on the chosen trivialization $\Psi$.

Let $(S, j)$ and $\left(S^{\prime}, j^{\prime}\right)$ be Riemman surfaces and let

$$
\tilde{u}:(S \backslash \Gamma, j) \rightarrow(\mathbb{R} \times M, \tilde{J}), \quad \tilde{v}:\left(S^{\prime} \backslash \Gamma^{\prime}, j^{\prime}\right) \rightarrow(\mathbb{R} \times M, \tilde{J})
$$

be finite energy $\tilde{J}$ holomorphic surfaces. We say that $(\tilde{u},(S, j), \Gamma)$ and $\left(\tilde{v},\left(S^{\prime}, j^{\prime}\right), \Gamma^{\prime}\right)$ are equivalent if there exists a biholomorphic map $\phi:(S, j) \rightarrow$ 
$\left(S^{\prime}, j^{\prime}\right)$ satisfying $\phi(\Gamma)=\Gamma^{\prime}$ and $\tilde{u}=\tilde{v} \circ \phi$. We denote by $[(\tilde{u},(S, j), \Gamma)]$ the equivalence class of $(\tilde{u},(S, j), \Gamma)$.

In Dra04, it is proved that the (equivalence classes of) finite energy spheres in the neighborhood of $[(\tilde{u},(S, j), \Gamma)]$, where $\tilde{u}$ is a somewhere injective finite energy surface, are described by a nonlinear Fredholm equation having Fredholm index equal to

$$
\operatorname{ind}(\tilde{u}):=\mu(\tilde{u})-\chi(S)+\# \Gamma .
$$

Due to the $\mathbb{R}$-action, the kernel of the linearized Fredholm operator is at least one-dimensional unless the image of $\tilde{u}$ is a cylinder over a periodic orbit, in which case $\pi \circ d u \equiv 0$.

The following result was proved for embedded finite energy surfaces in [HWZ99b] and generalized for somewhere injective finite energy surfaces in Dra04.

Theorem 2.20. There exists a residual set $\mathcal{J}_{\text {reg }} \subset \mathcal{J}(\xi, d \lambda)$ such that, if $\tilde{u}=(a, u): S \backslash \Gamma \rightarrow \mathbb{R} \times M$ is a somewhere injective finite energy surface for $\tilde{J} \in \mathcal{J}_{\text {reg }}$, then

$$
0 \leq \operatorname{ind}(\tilde{u}):=\mu(\tilde{u})-\chi(S)+\# \Gamma .
$$

If $\pi \circ d u$ is not identically zero, then

$$
1 \leq \operatorname{ind}(\tilde{u}):=\mu(\tilde{u})-\chi(S)+\# \Gamma .
$$

\subsection{Bubbling-off analysis}

This section follows mainly the exposition of [HSa11] and [HLSa15].

In this section, $\lambda$ is a nondegenerate tight contact form on $S^{3}$. We fix a Riemannian metric $g$ on $S^{3}$ and consider the Riemannian metric $g_{0}$ on $\mathbb{R} \times S^{3}$ defined by

$$
g_{0}=d a \otimes d a+\pi_{S^{3}}^{*} g,
$$

where $\pi_{S^{3}}: \mathbb{R} \times S^{3} \rightarrow S^{3}$ and $a: \mathbb{R} \times S^{3} \rightarrow \mathbb{R}$ are the projections onto the second and the first coordinates respectively. For any $(a, x) \in \mathbb{R} \times S^{3}$ and any linear map $L: \mathbb{C} \rightarrow T_{(a, x)}\left(\mathbb{R} \times S^{3}\right)$ we denote by $|L|$ the norm induced by the Euclidean inner product of $\mathbb{C}$ and the metric $g_{0}$.

\subsubsection{Elliptic Regularity and compactness}

Theorem 2.21 (Elliptic regularity). Let $(W, J)$ be an almost complex manifold and $(S, j)$ be a Riemannian surface without boundary. If $u: S \rightarrow W$ is 
a $C^{1}$ map and satisfies $\bar{\partial}_{J}(u)=0$, then $u$ is $C^{\infty}$.

Theorem 2.22. Let $(W, J)$ be an almost complex manifold and $(S, j)$ a closed Riemann surface. Let $\left\{U_{j}\right\}_{j \geq 1}$ be an increasing sequence of open sets in $S$ and $u_{j}: U_{j} \rightarrow W$ a sequence of $C^{\infty}$ pseudoholomorphic maps. Defining $U=\cup_{j} U_{j}$, assume that for all compact set $A \subset U$, there exists a compact set $K \subset W$ and a constant $C>0$ such that

$$
u_{j}(A) \subset K, \text { for } j \text { large and } \limsup _{j \rightarrow \infty}\left|d u_{j}\right|_{L^{\infty}(A)} \leq C
$$

Then there exists a $C^{\infty}$ map $u: U \rightarrow W$ satisfying $\bar{\partial}_{J}(u)=0$ and a subsequence $u_{j_{k}}$ such that

$$
u_{j_{k}} \rightarrow u \text { in } C_{l o c}^{\infty},
$$

that is, $u_{j_{k}} \rightarrow u$ uniformly with all derivatives on compact subsets of $U$.

Proofs of theorems 2.21 and 2.22 can be found in [HSa09.

\subsubsection{Bubbling}

Lemma 2.23 (Ekeland-Hofer). Let $(X, d)$ be a complete metric space and $f: X \rightarrow[0,+\infty)$ a continuous function. For any $\epsilon_{0}>0$ and $x_{0} \in X$, there exist $\epsilon_{0}^{\prime} \in\left(0, \epsilon_{0}\right]$ and $x_{0}^{\prime} \in \overline{B_{2 \epsilon_{0}}\left(x_{0}\right)}$ such that

$$
\left\{\begin{array}{l}
f\left(x_{0}^{\prime}\right) \epsilon_{0}^{\prime} \geq f\left(x_{0}\right) \epsilon_{0} \\
d\left(x, x_{0}^{\prime}\right) \leq \epsilon_{0}^{\prime} \Rightarrow f(x) \leq 2 f\left(x_{0}^{\prime}\right)
\end{array}\right.
$$

Proof. Suppose the claim is false, that is, there exist $x_{0} \in X$ and $\epsilon_{0}>0$ such that, for every $\epsilon^{\prime} \in\left(0, \epsilon_{0}\right]$ and $x^{\prime} \in \overline{B_{2 \epsilon_{0}}\left(x_{0}\right)}$, at least one of the following is true

- $f\left(x^{\prime}\right) \epsilon^{\prime}<f\left(x_{0}\right) \epsilon_{0}$

- $\exists x \in \overline{B_{\epsilon^{\prime}}\left(x^{\prime}\right)}$ such that $f(x)>2 f\left(x^{\prime}\right)$.

We will find a Cauchy sequence $\left\{x_{N}\right\}$ satisfying $f\left(x_{N}\right) \rightarrow \infty$, in contradiction to the fact that $X$ is complete. Taking $x^{\prime}=x_{0}$ and $\epsilon^{\prime}=\epsilon_{0}$, we find $x_{1} \in$ $\overline{B_{\epsilon_{0}}\left(x_{0}\right)}$ satisfying $f\left(x_{1}\right)>2 f\left(x_{0}\right)$. Assume we have $\left\{x_{0}, \ldots, x_{N}\right\}$ satisfying $d\left(x_{n+1}, x_{n}\right) \leq \epsilon_{0} 2^{-n}$ and $f\left(x_{n+1}\right)>2 f\left(x_{n}\right)$ for all $n=0, \ldots, N-1$. Since $d\left(x_{N}, x_{0}\right) \leq \epsilon_{0}\left(2^{-(N-1)}+\ldots,+2^{0}\right) \leq 2 \epsilon_{0}$, we have $x_{N} \in \overline{B_{2 \epsilon_{0}}\left(x_{0}\right)}$. Consider $x^{\prime}=x_{N}$ and $\epsilon^{\prime}=\epsilon_{0} 2^{-N}$. Since, by assumption, $f\left(x_{N}\right)>2^{N} f\left(x_{0}\right)$, we conclude there exists $x_{N+1}$ satisfying $d\left(x_{N+1}, x_{N}\right) \leq \epsilon_{0} 2^{-N}$ and $f\left(x_{N+1}\right)>$ $2 f\left(x_{N}\right)$. Thus $\left\{x_{N}\right\}$ is a Cauchy sequence and $f\left(x_{N}\right) \rightarrow \infty$, as $N \rightarrow \infty$. 
Proposition 2.24. Fix $J \in \mathcal{J}(\xi, d \lambda)$ and let $\tilde{J}$ be the induced almost complex structure on $\mathbb{R} \times S^{3}$ defined by formula (2.1). Let $\left\{U_{n}\right\}$ be an increasing sequence of open sets in $\mathbb{C}$ and let $\tilde{u}_{n}=\left(a_{n}, u_{n}\right): U_{n} \rightarrow \mathbb{R} \times S^{3}$ be a sequence of smooth maps satisfying $\bar{\partial}_{\tilde{J}}\left(\tilde{u}_{n}\right)=0, \forall n$ and $\sup _{n} E\left(\tilde{u}_{n}\right)=C<\infty$. Define $U=\cup_{n} U_{n}$ and assume there exits a sequence $z_{n} \in U_{n}$ such that $\left|d \tilde{u}_{n}\left(z_{n}\right)\right| \rightarrow$ $+\infty$ and $z_{n} \rightarrow z_{\infty} \in U$. Then there exist subsequences $\left\{\tilde{u}_{n_{k}}\right\}$ and sequences $z_{k}^{\prime} \rightarrow z_{\infty}, \delta_{k}, r_{k} \rightarrow 0^{+}$with $\frac{\delta_{k}}{r_{k}} \rightarrow 0^{+}$such that the family of maps

$$
\begin{aligned}
\tilde{v}_{k}: B_{\frac{1}{r_{k}}}(0) & \rightarrow \mathbb{R} \times S^{3} \\
z & \mapsto\left(a_{n_{k}}\left(z_{k}^{\prime}+\delta_{k} z\right)-a_{n_{k}}\left(z_{k}^{\prime}\right), u_{n_{k}}\left(z_{k}^{\prime}+\delta_{k} z\right)\right)
\end{aligned}
$$

converges in $C_{\text {loc }}^{\infty}$ to a $\tilde{J}$-holomorphic plane $\tilde{v}: \mathbb{C} \rightarrow \mathbb{R} \times M$ satisfying $0<$ $E(\tilde{v}) \leq C$ and $\sup _{\mathbb{C}}|d \tilde{v}|<\infty$.

Proof. The proof follows Proposition 3.67 of [HSa09]. Define $R_{n}=\left|d \tilde{u}_{n}\left(z_{n}\right)\right|$ and $\epsilon_{n}=R_{n}^{-\frac{1}{2}}$. By applying Lemma 2.23 to $\left|d \tilde{u}_{n}(z)\right|, z_{n}$ and $\epsilon_{n}$, we find $0<\epsilon^{\prime} \leq \epsilon_{n}$ and $z_{n}^{\prime}$ with $\left|z_{n}^{\prime}-z_{n}\right| \leq 2 \epsilon_{n}$ such that

$$
\left|d \tilde{u}_{n}\left(z_{n}^{\prime}\right)\right| \epsilon_{n}^{\prime} \geq\left|d \tilde{u}_{n}\left(z_{n}\right)\right| \epsilon_{n}=\epsilon_{n} R_{n}
$$

and

$$
\left|z-z_{n}^{\prime}\right| \leq \epsilon_{n}^{\prime} \Rightarrow\left|d \tilde{u}_{n}(z)\right| \leq 2\left|d \tilde{u}_{n}\left(z_{n}^{\prime}\right)\right|
$$

It follows that

$$
\sup _{\left|z-z_{n}^{\prime}\right| \leq \epsilon_{n}}\left|d \tilde{u}_{n}(z)\right| \leq 2\left|d \tilde{u}_{n}\left(z_{n}^{\prime}\right)\right| .
$$

Define $R_{n}^{\prime}=\left\|d \tilde{u}_{n}\left(z_{n}^{\prime}\right)\right\|$. Note that $\epsilon_{n}^{\prime} R_{n}^{\prime} \geq \epsilon_{n} R_{n} \rightarrow+\infty$ and define

$$
\begin{aligned}
\tilde{v}_{n}: B_{\epsilon_{n} R_{n}^{\prime}}(0) & \rightarrow \mathbb{R} \times S^{3} \\
z & \mapsto\left(a_{n}\left(z_{n}^{\prime}+\frac{z}{R_{n}^{\prime}}\right)-a_{n}\left(z_{n}^{\prime}\right), u_{n}\left(z_{n}^{\prime}+\frac{z_{n}^{\prime}}{R_{n}^{\prime}}\right)\right)
\end{aligned}
$$

If $z \in B_{\epsilon_{n}^{\prime} R_{n}^{\prime}(0)}$, using that the norm $|\cdot|$ is $\mathbb{R}$-invariant and 2.10 we get

$$
\left|d \tilde{v}_{n}(z)\right|=\left|d \tilde{u}_{n}\left(z_{n}^{\prime}+\frac{z}{R_{n}^{\prime}}\right)\right| \frac{1}{R_{n}^{\prime}} \leq 2, \forall n .
$$

Fixed $n_{0}$, using the mean value inequality and 2.11), and noting that $\tilde{v}_{n}(0) \in$ $\{0\} \times M$, we conclude that $\tilde{v}_{n}\left(B_{\epsilon_{n_{0}}^{\prime} R_{n_{0}}^{\prime}}(0)\right)$ is contained in a compact set. By Theorem 2.22, there is a subsequence $\tilde{v}_{n_{k}}$ and a $\tilde{J}$-holomorphic curve 
$\tilde{v}: \mathbb{C} \rightarrow \mathbb{R} \times M$, such that

$$
\tilde{v}_{n_{k}} \rightarrow \tilde{v} \quad \text { in } C_{l o c}^{\infty}
$$

Clearly $|d \tilde{v}|_{\mathbb{C}}<\infty$ and, by Fatou's Lemma, we get $E(\tilde{v}) \leq C$. Since $\left|d \tilde{v}_{n_{k}}(0)\right|=\lim _{k}\left|d \tilde{v}_{n_{k}}(0)\right|=1$, it follows that $E(\tilde{v})>0$.

Defining $\delta_{k}=\frac{1}{R_{n_{k}}^{\prime}}$ and $r_{k}=\frac{1}{\epsilon_{n_{k}} R_{n_{k}}^{\prime}}$, we have $\delta_{k}, r_{k} \rightarrow 0^{+}$and $\frac{\delta_{k}}{r_{k}} \rightarrow 0^{+}$. The family of maps $\tilde{v}_{k}:=\tilde{v}_{n_{k}}: B_{\frac{1}{r_{k}}}(0) \rightarrow \mathbb{R} \times S^{3}$ satisfies the statement of Proposition 2.24 .

\subsubsection{Germinating sequences}

Fix $C>0$. Since $\lambda$ is nondegenerate, there is just a finite number of orbits in $\mathcal{P}(\lambda)$ with period $\leq C$. Define $\sigma(C)$ as any real number satisfying

$$
0<\sigma(C)<\min \left\{T^{\prime},\left|T^{\prime}-T^{\prime \prime}\right|: T^{\prime} \neq T^{\prime \prime} \text { periods }, T^{\prime}, T^{\prime \prime} \leq C\right\} .
$$

Now we fix an arbitrary $J \in \mathcal{J}(\xi, d \lambda)$ and consider a sequence of $\tilde{J}_{-}$ holomorphic curves

$$
\tilde{v}_{n}=\left(b_{n}, v_{n}\right): B_{R_{n}}(0) \subset \mathbb{C} \rightarrow \mathbb{R} \times S^{3}
$$

satisfying

$$
\begin{array}{r}
R_{n} \rightarrow \infty, \quad R_{n} \in(0,+\infty] \\
E\left(\tilde{v}_{n}\right) \leq C, \forall n \\
\int_{B_{R_{n}}(0) \backslash \mathbb{D}} v_{n}^{*} d \lambda \leq \sigma(C), \forall n \\
\left\{b_{n}(2)\right\} \text { is uniformily bounded }
\end{array}
$$

Definition 2.25. Such a sequence $\tilde{v}_{n}$ of $\tilde{J}$-holomorphic curves satisfying 2.13-2.16 will be referred to as a germinating sequence.

Proposition 2.26. Let $\tilde{v}_{n}$ be a germinating sequence. Then there exists a finite set $\Gamma \subset \mathbb{D}$, a $\tilde{J}$-holomorphic map $\tilde{v}=(b, v): \mathbb{C} \backslash \Gamma \rightarrow \mathbb{R} \times S^{3}$ and a subsequence of $\tilde{v}_{n}$, still denoted by $\tilde{v}_{n}$, such that

$$
\tilde{v}_{n} \rightarrow \tilde{v} \text { in } C_{l o c}^{\infty}\left(\mathbb{C} \backslash \Gamma, \mathbb{R} \times S^{3}\right) .
$$

Also, $E(\tilde{v}) \leq C$.

Proof. Let $\Gamma_{0} \subset \mathbb{C}$ be the set of points $z \in \mathbb{C}$ such that there exists a 
subsequence $\tilde{v}_{n_{j}}$ and a sequence $\zeta_{j} \in B_{R_{n_{j}}}(0)$ with $\zeta_{j} \rightarrow z$ and

$$
\left|d \tilde{v}_{n_{j}}\left(\zeta_{l}\right)\right| \rightarrow \infty, j \rightarrow \infty
$$

If $\Gamma_{0}=\emptyset$, then by Theorem 2.22 , we find a $\tilde{J}$-holomorphic map $\tilde{v}: \mathbb{C} \rightarrow \mathbb{R} \times S^{3}$ such that, up to a subsequence, $\tilde{v}_{n} \rightarrow \tilde{v}$ in $C_{\text {loc }}^{\infty}\left(\mathbb{C}, \mathbb{R} \times S^{3}\right)$. In this case, $\Gamma=\emptyset$.

Now assume $\Gamma_{0} \neq \emptyset$. If $z_{0} \in \Gamma_{0}$, there exists a period $0<T_{0} \leq C$ and sequences $r_{j}^{0} \rightarrow 0^{+}, n_{j}^{0} \rightarrow \infty$ and $z_{j}^{0} \rightarrow z_{0}$ such that

$$
\lim _{j \rightarrow \infty} \int_{B_{r_{j}}\left(z_{j}^{0}\right)} v_{n_{j}^{0}}^{*} d \lambda \geq T_{0} .
$$

Indeed, by Proposition 2.24, there exists a subsequence $\left\{\tilde{v}_{n_{k}}\right\}$ and sequences $z_{k}^{\prime} \rightarrow z_{0}, r_{k}, \delta_{k} \rightarrow 0^{+}$, with $\frac{\partial_{k}}{r_{k}} \rightarrow 0^{+}$, such that the sequence of maps

$$
\begin{aligned}
\tilde{u}_{k}: B_{1 / r_{k}}(0) & \rightarrow \mathbb{R} \times S^{3} \\
z & \mapsto\left(b_{n_{k}}\left(z_{k}^{\prime}+\delta_{k} z\right)-b_{n_{k}}\left(z_{k}^{\prime}\right), v_{n_{k}}\left(z_{k}^{\prime}+\delta_{k} z\right)\right)
\end{aligned}
$$

converges in $C_{l o c}^{\infty}$ to a $\tilde{J}$-holomorphic plane $\tilde{u}: \mathbb{C} \rightarrow \mathbb{R} \times M$ satisfying $0<$ $E(\tilde{u}) \leq C$ and $\sup _{\mathbb{C}}|d \tilde{u}|<\infty$. Then there exists a period $T_{0} \leq C$ such that $T_{0}=\int_{\mathbb{C}} u^{*} d \lambda$. Using Fatou's Lemma, we obtain

$$
T_{0}=\int_{\mathbb{C}} u^{*} d \lambda \leq \liminf _{k \rightarrow \infty} \int_{B_{1 / r_{k}}(0)} u_{k}^{*} d \lambda=\liminf _{k \rightarrow \infty} \int_{B_{\delta_{k} / r_{k}}\left(z_{k}^{\prime}\right)} v_{n_{k}}^{*} d \lambda .
$$

Consider $\tilde{v}_{n_{j}^{0}}$ as the new sequence $\tilde{v}_{n}$. Now let $\Gamma_{1} \subset \mathbb{C} \backslash\left\{z_{0}\right\}$ be the set of points $z_{1} \neq z_{0}$ such that there exists a subsequence $\tilde{v}_{n_{j}}$ and sequence $\zeta_{j} \in B_{R_{n_{j}}}(0)$ with $\zeta \rightarrow z_{1}$ and $\left|d \tilde{v}_{n_{j}}\left(\zeta_{j}\right)\right| \rightarrow \infty$.

As before, if $\Gamma_{1}=\emptyset$, we have a $\tilde{J}$-holomorphic map $\tilde{v}: \mathbb{C} \backslash\left\{z_{0}\right\} \rightarrow \mathbb{R} \times S^{3}$ such that, up to subsequence, $\tilde{v}_{n} \rightarrow \tilde{v}$ in $C_{\text {loc }}^{\infty}\left(\mathbb{C} \backslash\left\{z_{0}\right\}, \mathbb{R} \times S^{3}\right)$. In this case, we define $\Gamma=\Gamma_{0}=\left\{z_{0}\right\}$.

If $\Gamma_{1} \neq \emptyset$ and $z_{1} \in \Gamma_{1}$, there exist a period $0<T_{1} \leq C$ and sequences $r_{j}^{1} \rightarrow 0, n_{j}^{1} \rightarrow \infty$ and $z_{j}^{1} \rightarrow z_{1}$ such that

$$
\lim _{j \rightarrow \infty} \int_{B_{r_{j}}\left(z_{j}^{1}\right)} v_{n_{j}^{1}}^{*} d \lambda \geq T_{1}
$$

Considering $\tilde{v}_{n_{j}^{1}}$ as the new sequence $\tilde{v}_{n}$, define $\Gamma_{2} \subset \mathbb{C} \backslash\left\{z_{0}, z_{1}\right\}$ as before. 
Repeating this argument, let $z_{i} \in \Gamma_{i} \subset \mathbb{C} \backslash\left\{z_{0}, \ldots, z_{i-1}\right\}$. Note that

$$
\begin{aligned}
C & \geq \lim _{n \rightarrow \infty} E\left(\tilde{v}_{n}\right) \geq \lim _{n \rightarrow \infty} \int_{\mathbb{C}} v_{n}^{*} d \lambda \\
& \geq \sum_{l=0}^{i} \lim _{j \rightarrow \infty} \int_{B_{r_{j}^{l}}\left(z_{j}^{l}\right)} v_{n_{j}^{l}}^{*} d \lambda \geq T_{0}+\cdots+T_{i}
\end{aligned}
$$

It follows that there exists $i_{0}$ such that $\Gamma_{i_{0}} \neq 0$ and $\Gamma_{i}=\emptyset$ for $i>i_{0}$.

We end up with a finite set $\Gamma=\left\{z_{0}, \ldots, z_{i_{0}}\right\}$ and a $\tilde{J}$-holomorphic map

$$
\tilde{v}: \mathbb{C} \backslash \Gamma \rightarrow \mathbb{R} \times S^{3}
$$

such that up to a subsequence,

$$
\tilde{v}_{n} \rightarrow \tilde{v} \text { in } C_{l o c}^{\infty} .
$$

It follows from $(2.15)$ that $\Gamma \subset \mathbb{D}$. The inequality $E(\tilde{v}) \leq C$ follows from (2.14) and Fatou's Lemma.

Definition 2.27. A $\tilde{J}$-holomorphic map $\tilde{v}: \mathbb{C} \backslash \Gamma \rightarrow \mathbb{R} \times S^{3}$ as in Proposition 2.26 is called a limit of the germinating sequence.

If $\Gamma \neq \emptyset$, then $\tilde{v}$ is non-constant. In this case, all the punctures $z=z_{i} \in \Gamma$ are negative and $\infty$ is a positive puncture. To prove this, define for any $\epsilon>0$

$$
m_{\epsilon}(z):=\int_{\partial B_{\epsilon}(z)} v^{*} \lambda
$$

Here $\partial B_{\epsilon}(z)$ is oriented counterclockwise. This is equivalent to use negative exponential coordinates as defined in Section 2.2 .

$$
m_{\epsilon}(z)=\int_{\partial B_{\epsilon}(z)} v^{*} \lambda=\lim _{n \rightarrow \infty} \int_{\partial B_{\epsilon}(z)} v_{n}^{*} \lambda=\lim _{n \rightarrow \infty} \int_{B_{\epsilon}(z)} v_{n}^{*} d \lambda .
$$

For $j$ large, $B_{r_{j}^{i}}\left(z_{j}^{i}\right)$, defined as in the proof of Proposition 2.26, is contained in $B_{\epsilon}(z)$. It follows that

$$
m_{\epsilon}(z)=\lim _{n \rightarrow \infty} \int_{B_{\epsilon}(z)} v_{n}^{*} d \lambda \geq \lim _{j \rightarrow \infty} \int_{B_{r_{j}}\left(z_{j}^{i}\right)} v_{n_{j}^{i}}^{*} d \lambda \geq T_{i}>0 .
$$

This implies that $\tilde{v}$ is non constant and the puncture $z$ is negative. Also, as a consequence of $0<E(\tilde{v})<\infty$, we know that $\tilde{v}$ has at least one positive puncture. Thus, $\infty$ is a positive puncture. 


\subsubsection{Soft-rescaling near a negative puncture}

Assume $\Gamma \neq \emptyset$ and let $\tilde{v}=(b, v): \mathbb{C} \backslash \Gamma \rightarrow \mathbb{R} \times M$ be a limit of a germinating sequence $\tilde{v}_{n}=\left(b_{n}, v_{n}\right)$. Let $z \in \Gamma$. We define the mass $m(z)$ of $z$ by

$$
m(z)=\lim _{\epsilon \rightarrow 0^{+}} m_{\epsilon}(z)=\lim _{\epsilon \rightarrow 0^{+}} \int_{\partial B_{\epsilon}(z)} v^{*} \lambda=T_{z}>\sigma(C)>0,
$$

where $T_{z}$ is the period of the asymptotic limit of $\tilde{v}$ at $z$.

Since $m_{\epsilon}(z)$ is a non-decreasing function of $\epsilon$, we can fix $\epsilon$ small enough so that

$$
0 \leq m_{\epsilon}(z)-m(z) \leq \frac{\sigma(C)}{2} .
$$

Choose sequences $z_{n} \in \overline{B_{\epsilon}(z)}$ and $0<\delta_{n}<\epsilon, \forall n$, so that

$$
\begin{gathered}
b_{n}\left(z_{n}\right) \leq b_{n}(\zeta), \forall \zeta \in B_{\epsilon}(z), \\
\int_{B_{\epsilon}(z) \backslash B_{\delta_{n}}\left(z_{n}\right)} v_{n}^{*} d \lambda=\sigma(C) .
\end{gathered}
$$

Since $z$ is a negative puncture, 2.19 implies that $z_{n} \rightarrow z$. Hence the existence of $\delta_{n}$ as in (2.20) follows from (2.17). We claim that $\lim \inf \delta_{n}=0$. Otherwise, we choose $0<\epsilon^{\prime}<\lim \inf \delta_{n} \leq \epsilon$. From (2.18), we get the contradiction

$$
\begin{aligned}
\frac{\sigma(C)}{2} & \geq m_{\epsilon}(z)-m(z) \geq m_{\epsilon}(z)-m_{\epsilon^{\prime}}(z) \\
& =\lim _{n \rightarrow \infty} \int_{B_{\epsilon}(z) \backslash B_{\epsilon^{\prime}}(z)} v_{n}^{*} d \lambda \\
& \geq \lim _{n \rightarrow \infty} \int_{B_{\epsilon}(z) \backslash B_{\delta_{n}}\left(z_{n}\right)} v_{n}^{*} d \lambda=\sigma(C) .
\end{aligned}
$$

Thus, we can assume $\delta_{n} \rightarrow 0$.

Now take any sequence $R_{n} \rightarrow+\infty$ satisfying

$$
\delta_{n} R_{n}<\frac{\epsilon}{2}
$$

and define the sequence of $\tilde{J}$-holomorphic maps $\tilde{w}_{n}=\left(c_{n}, w_{n}\right): B_{R_{n}}(0) \rightarrow$ $\mathbb{R} \times S^{3}$ by

$$
\tilde{w}_{n}(\zeta)=\left(b_{n}\left(z_{n}+\delta_{n} \zeta\right)-b_{n}\left(z_{n}+2 \delta_{n}\right), v_{n}\left(z_{n}+\delta_{n} \zeta\right)\right)
$$


It follows from 2.20$)$ that

$$
\int_{B_{R_{n}}(0) \backslash \mathbb{D}} w_{n}^{*} d \lambda \leq \sigma(C), \forall n .
$$

Moreover, by the definition of $\tilde{w}_{n}, E\left(\tilde{w}_{n}\right) \leq E\left(\tilde{v}_{n}\right) \leq C$ and $\tilde{w}_{n}(2) \in$ $\{0\} \times M$. Thus, $\tilde{w}_{n}$ is a germinating sequence.

Let $\tilde{w}=(c, w): \mathbb{C} \backslash \Gamma^{\prime} \rightarrow \mathbb{R} \times S^{3}$ be a limit of $\tilde{w}_{n}$, as in Proposition 2.26. If $\Gamma^{\prime} \neq \emptyset$, then $\tilde{w}$ is not constant. If $\Gamma^{\prime}=\emptyset$, then

$$
\begin{aligned}
\int_{\mathbb{D}} w^{*} d \lambda & =\lim _{n \rightarrow \infty} \int_{\mathbb{D}} w_{n}^{*} d \lambda=\lim _{n \rightarrow \infty} \int_{B_{\delta_{n}}\left(z_{n}\right)} v_{n}^{*} d \lambda \\
& =\lim _{n \rightarrow \infty}\left(\int_{B_{\epsilon}(z)} v_{n}^{*} d \lambda-\int_{B_{\epsilon}(z) \backslash B_{\delta_{n}}\left(z_{n}\right)} v_{n}^{*} d \lambda\right) \\
& =m_{\epsilon}(z)-\sigma(C) \\
& \geq T_{z}-\sigma(C)>0 .
\end{aligned}
$$

Thus $\tilde{w}$ is non-constant as well. From Fatou's Lemma we get $0<E(\tilde{w}) \leq C$. This also implies that the period of the asymptotic limits of $\tilde{w}$ are bounded by $C$.

Proposition 2.28. The asymptotic limit $P_{\infty}$ of $\tilde{w}$ at $\infty$ coincides with the asymptotic limit $P_{z}$ of $\tilde{v}$ at the negative puncture $z \in \Gamma$.

To prove Proposition 2.28, we need the following Lemma from [HWZ03].

Lemma 2.29. [HWZ03, Lemma 4.9] Consider constants $e>0$ and $C>$ 0 and let $\sigma(C)$ be as defined in 2.12). Identifying $S^{1}=\mathbb{R} / \mathbb{Z}$, let $\mathcal{W} \subset$ $C^{\infty}\left(S^{1}, S^{3}\right)$ be an open neighborhood of the set of periodic orbits $P=(x, T) \in$ $\mathcal{P}(\lambda)$ with $T \leq C$, viewed as maps $x_{T}: S^{1} \rightarrow S^{3}, x_{T}(t)=x(T t)$. We assume that $\mathcal{W}$ is $S^{1}$-invariant, meaning that $y(\cdot+c) \in \mathcal{W} \Leftrightarrow y \in \mathcal{W}, \forall c \in S^{1}$, and that each of the connected components of $\mathcal{W}$ contains at most one periodic orbit modulo $S^{1}$-reparametrizations. Then there exists a constant $h>0$ such that the following holds. If $\tilde{u}=(a, u):[r, R] \times S^{1} \rightarrow \mathbb{R} \times S^{3}$ is a $\tilde{J}$-holomorphic cylinder satisfying

$E(\tilde{u}) \leq C, \quad \int_{[r, R] \times S^{1}} u^{*} d \lambda \leq \sigma(C), \quad \int_{\{r\} \times S^{1}} u^{*} \lambda \geq e \quad$ and $r+h \leq R-h$, then each loop $t \in S^{1} \rightarrow u(s, t)$ is contained in $\mathcal{W}$ for all $s \in[r+h, R-h]$. Proof of Proposition 2.28. Let $\mathcal{W}$ be an open neighborhood of the set of 
periodic orbits $P=(x, T) \in \mathcal{P}(\lambda)$ with $T \leq C$ as in Lemma 2.29. Let $\mathcal{W}_{\infty}$ and $\mathcal{W}_{z}$ be connected components of $\mathcal{W}$ containing $P_{\infty}$ and $P_{z}$ respectively.

Since $\tilde{v}_{n} \rightarrow \tilde{v}$, we can choose $0<\epsilon_{0}<\epsilon$ small enough so that, if $0<\rho \leq \epsilon_{0}$ is fixed, then the loop

$$
t \in S^{1} \mapsto v_{n}\left(z_{n}+\rho e^{i 2 \pi t}\right)
$$

belongs to $\mathcal{W}_{z}$ for $n$ large. Since $\tilde{w}_{n} \rightarrow \tilde{w}$, we can choose $R_{0}>1$ large enough so that, if $R \geq R_{0}$ is fixed, then the loop

$$
t \in S^{1} \mapsto w_{n}\left(R e^{i 2 \pi t}\right)=v_{n}\left(z_{n}+\delta_{n} R e^{i 2 \pi t}\right)
$$

belongs to $\mathcal{W}_{\infty}$ for $n$ large.

By (2.17) and (2.20), we can show that

$$
e:=\liminf \int_{\partial B_{\delta_{n} R_{0}\left(z_{n}\right)}} v_{n}^{*} \lambda>0
$$

Consider, for each $n$, the $\tilde{J}$-holomorphic cylinder $\tilde{C}_{n}:\left[\frac{\ln R_{o} \delta_{n}}{2 \pi}, \frac{\ln \epsilon_{0}}{2 \pi}\right] \times S^{1} \rightarrow$ $\mathbb{R} \times S^{3}$, defined by $\tilde{C}_{n}(s, t)=\tilde{v}_{n}\left(z_{n}+e^{2 \pi(s+i t)}\right)$. It follows from 2.20 that

$$
\int_{\left[\frac{\ln R_{o} \delta_{n}}{2 \pi}, \frac{\ln \epsilon_{0}}{2 \pi}\right] \times S^{1}} C_{n}^{*} d \lambda \leq \sigma(C)
$$

for $n$ large. Using (2.23) and 2.24) and applying Lemma 2.29, we find $h>0$ so that the loop

$$
t \mapsto C_{n}(s, t)
$$

is contained in $\mathcal{W}$ for all $s \in\left[\frac{\ln R_{0} \delta_{n}}{2 \pi}+h, \frac{\ln \epsilon_{0}}{2 \pi}-h\right]$ and $n$ large. But

$$
C_{n}\left(\frac{\ln \epsilon_{0}}{2 \pi}-h, t\right)=v_{n}\left(z_{n}+\epsilon_{0} e^{-2 \pi h} e^{2 \pi i t}\right) \in \mathcal{W}_{z}
$$

for all $n$ large and

$$
C_{n}\left(\frac{\ln R_{0} \delta_{n}}{2 \pi}+h, t\right)=v_{n}\left(z_{n}+R_{0} \delta_{n} e^{2 \pi h} e^{2 \pi t}\right) \in \mathcal{W}_{\infty}
$$

for all $n$ large. Thus $\mathcal{W}_{\infty}=\mathcal{W}_{z}$ and $P_{\infty}=P_{z}$.

Proposition 2.30. Either

- $\int_{\mathbb{C} \backslash \Gamma^{\prime}} w^{*} d \lambda>0$ or 
- $\int_{\mathbb{C} \backslash \Gamma^{\prime}} w^{*} d \lambda=0$ and $\# \Gamma^{\prime} \geq 2$.

Proof. If $\Gamma^{\prime} \neq \emptyset$, then $0 \in \Gamma^{\prime}$. This fact follows from $c_{n}(0)=\inf c_{n}\left(B_{R_{n}}\right)$ and the fact that the punctures in $\Gamma^{\prime}$ are negative. Arguing by contradiction, assume

$$
\int_{\mathbb{C} \backslash \Gamma^{\prime}} w^{*} d \lambda=0 \text { and } \# \Gamma^{\prime}=1
$$

Thus, $\Gamma^{\prime}=\{0\}$. By Theorem 2.6, there exists a polynomial $p: \mathbb{C} \rightarrow \mathbb{C}$ and an orbit $P=(x, T)$ such that $\Gamma^{\prime}=p^{-1}(0)$ and $\tilde{v}=F_{P} \circ p$, where $F_{P}: \mathbb{C} \backslash\{0\} \rightarrow \mathbb{R} \times S^{3}$ is defined by $F_{P}\left(\zeta=e^{2 \pi(s+i t)}\right)=(T s, x(T t))$. Since 0 is the only root of $p$, we have $p(\zeta)=A \zeta^{n}$, for some $A \neq 0$ and $n \geq 1$. It follows that $P^{n}=P_{z}$, where $P_{z}=\left(x_{z}, T_{z}\right)$ is the asymptotic limit of $\tilde{v}$ at $z$, that coincides with the asymptotic limit of $\tilde{w}$ at $\infty$, and

$$
\tilde{w}\left(\zeta=e^{2 \pi(s+i t)}\right)=F_{P}\left(A e^{2 \pi n(s+i t)}\right)=\left(T_{z}\left(s+\frac{\log A}{2 \pi n}\right), x_{z}\left(T_{z} t\right)\right) .
$$

Thus, we have the contradiction

$$
\begin{aligned}
m(z) & =T_{z}=\int_{\partial \mathbb{D}} w^{*} \lambda=\lim _{n \rightarrow \infty} \int_{\partial \mathbb{D}} w_{n}^{*} \lambda=\lim _{n \rightarrow \infty} \int_{\partial B_{\epsilon}(z)} v_{n}^{*} \lambda-\sigma(C) \\
& =\int_{\partial B_{\epsilon}(z)} v^{*} \lambda-\sigma(C) \leq m(z)-\frac{\sigma(C)}{2}
\end{aligned}
$$

Here we have used (2.18), 2.20) and (2.22).

\subsubsection{Bubbling-off tree}

Consider a finite rooted tree ${ }^{2} \mathcal{T}=(E,\{r\}, V)$, with edges oriented away from the root, and a finite set $\mathcal{U}$ of finite energy $\tilde{J}$-holomorphic spheres. The pair $\mathcal{B}=(\mathcal{T}, \mathcal{U})$ is called a bubbling-off tree if it satisfies the following properties

\footnotetext{
${ }^{2} \mathrm{~A}$ tree $\mathcal{T}=(E, V)$ is a connected graph with no cycles. A rooted tree is a tree $T=(V, E)$ with a distinguished vertex $r \in V$, called the root of $\mathcal{T}$. We denote a rooted tree $\mathcal{T}=(V, E)$ with root $r \in V$ by $\mathcal{T}=(V,\{r\}, E)$. The edges of a rooted tree have a natural orientation away from the root. Consider a rooted tree $\mathcal{T}=(V,\{r\}, E)$ with edges oriented away from the root. Each vertex $v$ distinct from the root has a unique incoming edge $e=(u, v)$ and possibly many outgoing edges $\left\{f_{i}=\left(v, w_{i}\right)\right\}, i=1, \ldots, N$. We call the vertex $u$ the parent of $v$ and the vertices $w_{i}, i=1, \ldots, N$ the children of $v$. A leaf is a vertex with no children. There is a level structure on $V$ defined by the following: The level of a vertex is the minimal number of edges necessary to reach the root plus one.
} 
- There is a bijective correspondence between vertices $q \in V$ and finiteenergy punctured spheres $\tilde{u}_{q}: \mathbb{C} \backslash \Gamma_{q} \rightarrow \mathbb{R} \times S^{3} \in \mathcal{U}$;

- Each sphere $\tilde{u}_{q}$ has exactly one positive puncture at $\infty$ and $0 \leq \# \Gamma_{q} \leq$ $\infty$ negative punctures, where $\Gamma_{q}$ is the set of negative punctures of $\tilde{u}_{q}$;

- If the vertex $q$ is not the root then $q$ has an incoming edge $e$ from a vertex $q^{\prime}$, and $\# \Gamma_{q}$ outgoing edges $f_{1}, \ldots, f_{\# \Gamma_{q}}$ to vertices $p_{1}, \ldots, p_{\# \Gamma_{q}}$ of $\mathcal{T}$, respectively. The edge $e$ is associated to the positive puncture of $\tilde{u}_{q}$ and the edges $f_{1}, \ldots, f_{\# \Gamma_{q}}$ are associated to the negative punctures of $\tilde{u}_{q}$. The asymptotic limit of $\tilde{u}_{q}$ at its positive puncture coincides with the asymptotic limit of $\tilde{u}_{q^{\prime}}$ at its negative puncture associated to $e$. In the same way, the asymptotic limit of $\tilde{u}_{q}$ at a negative puncture corresponding to $f_{i}$ coincides with the asymptotic limit of $\tilde{u}_{p_{i}}$ at its unique positive puncture;

- If the $d \lambda$-area of $\tilde{u}_{q}$ vanishes then $\# \Gamma_{q} \geq 2$.

The following Theorem is a simpler case of the SFT Compactness theorem from $\left[\mathrm{BEH}^{+} 03\right]$.

Theorem 2.31. Let $\left\{\tilde{u}_{n}=\left(a_{n}, u_{n}\right)\right\}$ be a germinating sequence with a nonconstant limit $\tilde{u}: \mathbb{C} \backslash \Gamma \rightarrow \mathbb{R} \times S^{3}$. Up to a subsequence of $\tilde{u}_{n}$, still denoted by $\tilde{u}_{n}$, there exists a bubbling-off tree $\mathcal{B}=(\mathcal{T}, \mathcal{U})$ with the following properties

- $\tilde{u}_{r}=\tilde{u}$, where $r$ is the root of the tree $\mathcal{T}$.

- For every vertex $q$ of $\mathcal{T}$ there exist sequences $z_{n}^{q}, \delta_{n}^{q} \in \mathbb{C}$ and $c_{n}^{q} \in \mathbb{R}$ such that

$$
\tilde{u}_{n}\left(z_{n}^{q}+\delta_{n}^{q} \cdot\right)+c_{n}^{q} \rightarrow \tilde{u}_{q}(\cdot) \text { in } C_{\text {loc }}^{\infty}\left(\mathbb{C} \backslash \Gamma_{q}\right) \text { as } n \rightarrow \infty \text {. }
$$

Here $\tilde{u}+c:=(a+c, u)$, where $\tilde{u}=(a, u)$ and $c \in \mathbb{R}$.

Proof. After a selection of a subsequence, we can assume $\tilde{u}_{n} \rightarrow \tilde{u}$ in $C_{\text {loc }}^{\infty}(\mathbb{C} \backslash$ $\left.\Gamma, \mathbb{R} \times S^{3}\right)$. We start with a tree containing just the root $r$ as a vertex and let $\tilde{u}_{r}=\tilde{u}$. If $\Gamma=\emptyset$, we define $\mathcal{T}=(\{r\}, r, \emptyset)$ and $\mathcal{B}=\left(\mathcal{T},\left\{\tilde{u}_{r}\right\}\right)$ and we have finished the construction of the bubbling-off tree.

Otherwise, let $z \in \Gamma$. As in Section 2.3.4, we define a germinating sequence $\left\{\tilde{w}_{z, n}\right\}$ by 2.21. Let $\tilde{w}_{z}: \mathbb{C} \backslash \Gamma_{z} \rightarrow \mathbb{R} \times M$ be a limit of the germinating sequence $\left\{\tilde{w}_{z, n}\right\}$. By Proposition 2.28 , the asymptotic limit of $\tilde{u}$ at the negative puncture $z$ coincides with the asymptotic limit of $\tilde{w}_{z}$ at $\infty$. By Proposition 2.30, if the $d \lambda$-area of $\tilde{w}_{z}$ vanishes, then $\# \Gamma_{z} \geq 2$. We add a vertex $q_{z}$ to the tree, an edge $f_{z}$ from $r$ to $q_{z}$ and let $\tilde{u}_{q_{z}}=\tilde{w}_{z}$. We do the 
same for all $z \in \Gamma$. If $\Gamma_{z}=\emptyset$ for all $z \in \Gamma$ we have finished the construction and define $\mathcal{T}=\left(\{r\} \cup\left\{q_{z}\right\}_{z \in \Gamma_{z}}, r,\left\{f_{z}\right\}_{z \in \Gamma}\right)$. Otherwise, we continue this process for every puncture $\zeta_{z} \in \Gamma_{z}$, for every $z \in \Gamma$.

By Proposition 2.30, the periods of the asymptotic limits strictly decreases when going down the tree. Since $T,\left|T-T^{\prime}\right|>\sigma(C)>0$, for $T, T^{\prime}$ periods and $T \neq T^{\prime}$, this process has to finish after a finite number of steps.

\subsubsection{Estimating Conley-Zehnder indices}

Remark 2.32. The tight contact structure $\xi_{0}$ on $S^{3}$ is a trivial symplectic bundle. We will fix a global symplectic trivialization $\Psi: \xi_{0} \rightarrow S^{3} \times \mathbb{R}^{2}$ and compute the index wind ${ }_{\infty}$ defined by $(2.7)$ and the Conley-Zender index $\mu$ with respect to this trivialization. We will denote $\operatorname{wind}_{\infty}(\cdot, \cdot)=\operatorname{wind}_{\infty}(\cdot, \cdot, \Psi)$ and $\mu(\cdot)=\mu(\cdot, \Psi)$. Recall that on $\left(S^{3}, \xi_{0}\right)$, the index wind $\infty_{\infty}$ and the ConleyZehnder index do not depend on the chosen global trivialization of $\xi_{0}$.

Lemma 2.33. Let $\tilde{v}:(S \backslash \Gamma, j) \rightarrow \mathbb{R} \times S^{3}$ be a finite energy curve and let $z \in \Gamma$ be a puncture with asymptotic limit $P=(x, T)$. Define $\nu_{P}^{\text {pos }}$ and $\nu_{P}^{\text {neg }}$ by (1.13) and (1.14 respectively. If $\pi \cdot d v$ does not vanish identically, then

(1) $\operatorname{wind}_{\infty}(\tilde{v}, z) \leq \operatorname{wind}\left(\nu^{n e g}\right)$ if $z$ is a positive puncture.

(2) $\operatorname{wind}_{\infty}(\tilde{v}, z) \geq \operatorname{wind}\left(\nu^{\text {pos }}\right)$ if $z$ is a negative puncture.

The proof follows immediately from the definition of wind ${ }_{\infty}$, Proposition 1.15 and Theorem 2.11.

The following Lemma will be useful later.

Lemma 2.34. Let $\tilde{u}=(a, u): \mathbb{C} \backslash \Gamma \rightarrow \mathbb{R} \times S^{3}$ be a finite energy $\tilde{J}_{-}$ holomorphic curve such that every puncture in $\Gamma$ is negative, $\pi \circ$ du does not vanish identically and for every asymptotic limit $P$ of $\tilde{u}, \mu(P) \in\{1,2,3\}$. Then $\operatorname{wind}_{\pi}(\tilde{u})=0$ and for all $z \in \Gamma \cup\{\infty\}$, $\operatorname{wind}_{\infty}(z)=1$.

Proof. By the definition of the Conley-Zehnder index in (1.15), for every asymptotic limit $P$ of $\tilde{u}$, one of the following options hold

$$
\begin{aligned}
& \mu(P)=1 \Rightarrow \operatorname{wind}\left(\nu_{P}^{\text {neg }}\right)=0, \quad \operatorname{wind}\left(\nu_{P}^{\text {pos }}\right)=1 \\
& \mu(P)=2 \Rightarrow \operatorname{wind}\left(\nu_{P}^{\text {neg }}\right)=1, \quad \operatorname{wind}\left(\nu_{P}^{\text {pos }}\right)=1 \\
& \mu(P)=3 \Rightarrow \operatorname{wind}\left(\nu_{P}^{\text {neg }}\right)=1, \quad \operatorname{wind}\left(\nu_{P}^{\text {pos }}\right)=2
\end{aligned}
$$

Then, by the definition of wind ${ }_{\infty}$, if $P$ is the asymptotic limit of $\tilde{u}$ at $z$ we have

$$
\begin{aligned}
& \operatorname{wind}_{\infty}(z) \leq \operatorname{wind}\left(\nu_{P}^{\text {neg }}\right) \leq 1, \text { if } z=\infty \\
& \left.\operatorname{wind}_{\infty}(z) \geq \operatorname{wind}\left(\nu_{P}^{\text {pos }}\right)\right) \geq 1, \text { if } z \in \Gamma .
\end{aligned}
$$


It follows that $\operatorname{wind}_{\infty}(\tilde{u}) \leq 1-\# \Gamma$. Since $\operatorname{wind}_{\pi}(\tilde{u}) \geq 0$, we have

$$
0 \leq \operatorname{wind}_{\pi}(\tilde{u})=\operatorname{wind}_{\infty}(\tilde{u})-1+\# \Gamma \leq 0 .
$$

Thus $\operatorname{wind}_{\pi}=0$ and $\operatorname{wind}_{\infty}(\tilde{u})=1-\# \Gamma$. Using (2.27), we conclude

$$
0 \geq \operatorname{wind}_{\infty}(\infty)-1=\sum_{z \in \Gamma} \operatorname{wind}_{\infty}(z)-\# \Gamma=\sum_{z \in \Gamma} \operatorname{wind}_{\infty}(z)-1 \geq 0 .
$$

Then $\operatorname{wind}_{\infty}(z)=1$, for all $z \in \Gamma \cup\{\infty\}$.

Now we present some results of [HSa11] that will be useful later.

Lemma 2.35. [HSa11, Lemma 3.3] Let $\Gamma=\left\{z_{1}, \cdots, z_{N}\right\} \subset \mathbb{C}$ be non-empty and finite, and let $\tilde{u}=(a, u): \dot{\mathbb{C}}=\mathbb{C} \backslash \Gamma \rightarrow \mathbb{R} \times S^{3}$ be a finite energy surface with exactly one positive puncture at $\infty$ and negative punctures at the points of $\Gamma$. If $\tilde{u}$ is asymptotic to $P_{\infty}$ at $\infty$ and is asymptotic to $P_{j}$ at $z_{j}$, for $j=1, \ldots, N$, then the following assertions are true:

$$
\begin{aligned}
& \text { i. } \int_{\dot{\mathbb{C}}} u^{*} d \lambda>0 \text { and } \mu\left(P_{\infty}\right) \leq 1 \Rightarrow \exists j \in 1, \ldots, n \text { such that } \mu\left(P_{j}\right) \leq 1 . \\
& \text { ii. } \int_{\dot{\mathbb{C}}} u^{*} d \lambda=0 \text { and } \mu\left(P_{\infty}\right)=1 \Rightarrow \mu\left(P_{j}\right)=1, \forall j=1, \ldots, N . \\
& \text { iii. } \int_{\dot{\mathbb{C}}} u^{*} d \lambda=0 \text { and } \mu\left(P_{\infty}\right) \leq 0 \Rightarrow \mu\left(P_{j}\right) \leq 0, \forall j=1, \ldots, N .
\end{aligned}
$$

Proof. Let $\nu_{j}^{\text {pos }}>0>\nu_{j}^{\text {neg }}$ be the special eigenvalues of the operators $A_{P_{j}}$, for $j=1, \ldots, N$, as defined in (1.14) and (1.13). In the same way, let $\nu_{\infty}^{\text {pos }}>0>\nu_{\infty}^{\text {neg }}$ be the special eigenvalues of the operator $A_{P_{\infty}}$. Suppose, by contradiction, that $\mu\left(P_{j}\right) \geq 2, \forall j=1, \ldots, N, \mu\left(P_{\infty}\right) \leq 1$ and $\pi \circ d u$ does not vanish identically. It follows from $(1.15)$ and Lemma 2.33 that

$$
\begin{aligned}
& \operatorname{wind}_{\infty}(\tilde{u}, \infty) \leq \operatorname{wind}\left(\nu_{\infty}^{\text {neg }}\right) \leq 0 \\
& \operatorname{wind}_{\infty}\left(\tilde{u}, z_{j}\right) \geq \operatorname{wind}\left(\nu_{j}^{\text {pos }}\right) \geq 1, \forall j=1, \ldots, N
\end{aligned}
$$

Theorem 2.17 implies that

$$
\begin{aligned}
1-N & \leq \operatorname{wind}_{\pi}(\tilde{u})+1-N \\
& =\operatorname{wind}_{\pi}(\tilde{u})+\chi_{S^{2}}-(N+1) \\
& =\operatorname{wind}_{\infty}(\tilde{u}) \\
& =\operatorname{wind}_{\infty}(\tilde{u}, \infty)-\sum_{j=1}^{n} \operatorname{wind}_{\infty}\left(\tilde{u}, z_{j}\right) \\
& \leq 0-N
\end{aligned}
$$


which is a contradiction. Assertion $i$. is proved. Now assume $\pi \circ d u$ vanishes identically. If $P_{\infty}=\left(x_{\infty}, T_{\infty}\right)$, denote by $\tau>0$ the minimal positive period of the Reeb trajectory $x_{\infty}$. Theorem 2.6 implies that each orbit $P_{j}$ is of the form $P_{j}=\left(x_{\infty}, k_{j} \tau\right)$ for some integer $k_{j} \geq 1$ and $T_{\infty}=\sum_{j=1}^{N} k_{j} \tau$. It follows from Lemma 1.9 that

$$
\begin{aligned}
& \mu\left(P_{\infty}\right)=1 \Rightarrow \mu\left(P_{j}\right)=1, \forall j=1, \ldots, N \\
& \mu\left(P_{\infty}\right) \leq 0 \Rightarrow \mu\left(P_{j}\right) \leq 0, \forall j=1, \ldots, N
\end{aligned}
$$

Lemma 2.36. [HSa11, Lemma 3.4] Let $\left\{z_{1}, \ldots, z_{N}\right\}, \tilde{u}=(a, u), P_{\infty}, P_{1}, \ldots P_{N}$ be as in the statement of Lemma 2.35. Assume that at least one of the following assertions is true

i. $\mu\left(P_{j}\right) \geq 2, \forall j=1, \ldots, N, \int_{\dot{\mathbb{C}}} u^{*} d \lambda>0$ and $\operatorname{wind}_{\infty}(\tilde{u}, \infty) \leq 1$.

ii. $\mu\left(P_{j}\right) \geq 2, \forall j=1, \ldots, N$, and $\mu\left(P_{\infty}\right) \leq 2$.

Then $\mu\left(P_{j}\right)=2, \forall j=1, \ldots, N$.

Proof. Assume $i$. As in the proof of Lemma 2.35, it follows from the definition of $\operatorname{wind}_{\infty}$ and 1.15 that $\operatorname{wind}_{\infty}\left(\tilde{u}, z_{j}\right) \geq 1$, for $j=1, \ldots, N$. Suppose, by contradiction, that there exists $j_{0}$ such that $\mu\left(P_{j_{0}}\right) \geq 3$. Then, again from the definition of wind $_{\infty}$ and $(1.15)$, we have $\operatorname{wind}_{\infty}\left(\tilde{u}, z_{j_{0}}\right) \geq 2$. Consequently,

$$
\begin{aligned}
1-N & =\chi\left(S^{2}\right)-(N+1) \\
& \leq \operatorname{wind}_{\pi}(\tilde{u})+\chi\left(S^{2}\right)-(N+1) \\
& =\operatorname{wind}_{\infty}(\tilde{u}) \\
& =\operatorname{wind}_{\infty}\left(\tilde{u}, \infty-\sum_{j=1}^{N} \operatorname{wind}_{\infty}\left(\tilde{u}, z_{j}\right)\right. \\
& \leq 1-(N-1)-2 \\
& =-N
\end{aligned}
$$

a contradiction.

Now assume $i$. If $\int_{\dot{\mathbb{C}}} u^{*} d \lambda>0$, by the definition of wind ${ }_{\infty}$ and equation 1.15, $\mu\left(P_{\infty}\right) \leq 2$ implies $\operatorname{wind}_{\infty}(\tilde{u}, \infty) \leq 1$. Then $i$. holds. If $\int_{\dot{\mathbb{C}}} u^{*} d \lambda=0$, $P_{\infty}=\left(x_{\infty}, T_{\infty}\right)$ and $\tau$ is the minimal period of $x_{\infty}$, then it follows from Theorem 2.6 that each orbit $P_{j}$ is of the form $P_{j}=\left(x_{\infty}, k_{j} \tau\right)$ for integers $k_{j} \geq 1$ and $T_{\infty}=\sum_{j=1}^{N} k_{j} \tau$. Lemma 1.9 implies that $\mu\left(P_{j}\right) \in\{1,2\}$ for $j=1, \ldots, N$. Our assumption implies $\mu\left(P_{j}\right)=2, \forall j=1, \ldots, N$. 
Lemma 2.37. [HSa11, Lemma 3.9] Let $\tilde{v}=(b, v)$ be a non-constant limit of a germinating sequence $\left\{\tilde{v}_{n}=\left(b_{n}, v_{n}\right)\right\}$. Then the asymptotic limit $P_{\infty}$ at the (unique) positive puncture of $\tilde{v}$ satisfies $\mu\left(P_{\infty}\right) \geq 2$.

Proof. Following Theorem 2.31, let $\mathcal{B}=(\mathcal{T}, \mathcal{U})$ be the bubbling-off tree associated with the germinating sequence $\tilde{v}_{n}$ and the limit $\tilde{v}$, so that if $r$ is the root of $\mathcal{T}, \tilde{u}_{r}=\tilde{v}$. Suppose, by contradiction, that $\tilde{u}_{r}: \mathbb{C} \backslash \Gamma_{r} \rightarrow \mathbb{R} \times S^{3}$ is asymptotic to $P_{\infty}$ at its positive puncture $\infty$ and $\mu\left(P_{\infty}\right) \leq 1$. If $\Gamma_{r}=\emptyset$, then, by the formula (1.15) and Lemma 2.33, $\tilde{u}_{r}$ is a finite energy plane satisfying

$$
\operatorname{wind}_{\infty}\left(\tilde{u}_{r}, \infty\right) \leq \operatorname{wind}\left(\nu_{P_{\infty}}^{n e g}\right) \leq 0 .
$$

By Theorem 2.17, we have $\operatorname{wind}_{\pi}\left(\tilde{u}_{r}\right)<0$, a contradiction. If $\Gamma_{r} \neq \emptyset$, we can use Lemma 2.35 to find a negative puncture $z \in \Gamma_{r}$ and a closed Reeb orbit $P_{1}$ such that $\tilde{u}_{r}$ is asymptotic to $P_{1}$ at $z$ and $\mu\left(P_{1}\right) \leq 1$. Thus, if $q$ is the vertex immediately below $r$, corresponding to the puncture $z$, then the asymptotic limit of $\tilde{u}_{q}: \mathbb{C} \backslash \Gamma_{q} \rightarrow \mathbb{R} \times S^{3}$ at its unique positive puncture is $P_{1}$. If $q$ is a leaf, we have a contradiction. If $q$ is not a leaf, we can use Lemma 2.35 again to find a negative puncture $z \in \Gamma_{q}$ and a closed Reeb orbit $P_{2}$ such that $\tilde{u}_{q}$ is asymptotic to $P_{2}$ at $z$ and $\mu\left(P_{2}\right) \leq 1$. Since the tree has a finite number of vertices, continuing this process we end up finding a leaf $l$ so that $\tilde{u}_{l}: \mathbb{C} \rightarrow \mathbb{R} \times S^{3}$ is a finite energy plane with asymptotic limit $P_{N}$ satisfying $\mu\left(P_{N}\right) \leq 1$, which implies $\operatorname{wind}_{\pi}\left(\tilde{u}_{l}\right)<0$, a contradiction.

Proposition 2.38. [HSa11, Proposition 3.10] Let $\left\{\tilde{v}_{n}=\left(b_{n}, v_{n}\right)\right\}$ be a germinating sequence and $\tilde{v}=(b, v): \mathbb{C} \backslash \Gamma \rightarrow \mathbb{R} \times S^{3}$ a non-constant limit of $\tilde{v}_{n}$. Assume that at least one of the following holds:

(i) $\Gamma \neq \emptyset, \int_{\mathbb{C} \backslash \Gamma} v^{*} d \lambda>0$ and $\operatorname{wind}_{\infty}(\tilde{v}, \infty) \leq 1$;

(ii) $\tilde{v}$ is asymptotic at $\infty$ to some Reeb orbit $P_{\infty}$ satisfying $\mu\left(P_{\infty}\right) \leq 2$.

Then there exists a Reeb orbit $P_{0}=\left(x_{0}, T_{0}\right)$ and a finite energy plane $\tilde{u}_{0}=$ $\left(a_{0}, u_{0}\right): \mathbb{C} \rightarrow \mathbb{R} \times S^{3}$, satisfying:

(1) $u_{0}: \mathbb{C} \rightarrow S^{3}$ is an immersion transversal to the Reeb vector field;

(2) $\mu\left(P_{0}\right)=2$ and $\tilde{u}_{0}$ is asymptotic to $P_{0}$ at the puncture $\infty$;

(3) if some Reeb orbit $P=(x, T)$ satisfies $v_{n}(\mathbb{C}) \cap x(\mathbb{R})=\emptyset \forall n$, then $u_{0}(\mathbb{C}) \cap x(\mathbb{R})=\emptyset$. If in addition $P$ is simply covered and satisfies $\mu(P) \geq 3$, then $\overline{u_{0}(\mathbb{C})} \cap x(\mathbb{R})=\emptyset$. 
Proof. Following Theorem 2.31, let $\mathcal{B}=(\mathcal{T}, \mathcal{U})$ be the bubbling-off tree associated with the germinating sequence $\tilde{v}_{n}$ and the limit $\tilde{v}$, so that if $r$ is the root of $\mathcal{T}$, then $\tilde{u}_{r}=\tilde{v}$. To prove Proposition 2.38, we need the following lemma.

Lemma 2.39. If a vertex $q$ of $\mathcal{T}$ is not the root and $\tilde{u}_{q}$ is asymptotic to the closed Reeb orbit $P$ at its (unique) positive puncture, then $\mu(P)=2$.

Proof. Let $\Gamma$ be the set of negative punctures of $\tilde{v}$. Lemma 2.37 implies that $\mu\left(P_{z}\right) \geq 2, \forall z \in \Gamma$, where $P_{z}$ is the asymptotic limit of $\tilde{v}$ at $z$. Using Lemma 2.36 we conclude that $\mu\left(P_{z}\right)=2 \forall z \in \Gamma$. The orbits $P_{z}$ are precisely the asymptotic limits at the positive punctures of the curves in the second level of the bubbling-off tree. We can apply Lemmas 2.37 and 2.36 to the curves in the second level of the tree to conclude that all negative asymptotic limits of these curves have Conley-Zenhder index equal to 2 . We can repeat this argument inductively on each level of the tree to conclude the proof.

First assume that $\Gamma=\emptyset$. Then we are in case (ii): $\tilde{v}: \mathbb{C} \rightarrow \mathbb{R} \times S^{3}$ is a finite energy plane and its asymptotic limit satisfies $\mu\left(P_{\infty}\right) \leq 2$. By Theorem 2.17. Lemma 2.33 and the formula (1.15), we have

$$
0 \leq \operatorname{wind}_{\pi}(\tilde{v})=\operatorname{wind}_{\infty}(\tilde{v}, \infty)-1 \leq \frac{1}{2} \mu\left(P_{\infty}\right)-1
$$

so that $\mu\left(P_{\infty}\right)=2$ and $\operatorname{wind}_{\pi}(\tilde{v})=0$. We set $\tilde{u}_{0}=\tilde{v}$ and $P_{0}=P_{\infty}$. As a consequence of the definition of wind $_{\pi}$, we conclude that

$$
\mathbb{R} R_{\lambda u_{0}(z)} \oplus d u_{0}(z)\left(T_{z} \mathbb{C}\right)=T_{u_{0}(z)} S^{3}, \forall z \in \mathbb{C}
$$

proving that $u_{0}$ is an immersion transverse to $R_{\lambda}$.

Now assume $\Gamma \neq 0$. The tree $\mathcal{T}$ has a leaf $q$ distinct from the root $r$. We set $\tilde{u}_{0}:=\tilde{u}_{q}$. By Lemma 2.39, we know that

$$
\tilde{u}_{0}=\left(a_{0}, u_{0}\right): \mathbb{C} \rightarrow \mathbb{R} \times S^{3}
$$

is a finite energy plane asymptotic to a closed Reeb orbit satisfying $\mu\left(P_{0}\right)=2$. Now we show that $u_{0}$ is an immersion transverse to the Reeb vector field. By Theorem 2.17, Lemma 2.33 and the formula (1.15), we have

$$
0 \leq \operatorname{wind}_{\pi}\left(\tilde{u}_{0}\right)=\operatorname{wind}_{\infty}\left(\tilde{u}_{0}, \infty\right)-1 \leq \frac{1}{2} \mu\left(P_{0}\right)-1=0,
$$

so that $\operatorname{wind}_{\pi}\left(\tilde{u}_{0}\right)=0$. As a consequence of the definition of $\operatorname{wind}_{\pi}$, we conclude that $\tilde{u}_{0}$ satisfies 2.28$)$, proving that $u_{0}$ is an immersion transverse 
to $R_{\lambda}$.

Thus, in both cases, we found a finite energy plane $\tilde{u}_{0}=\left(a_{0}, u_{0}\right): \mathbb{C} \rightarrow$ $\mathbb{R} \times S^{3}$ satisfying (1) and (2).

Now we prove that (3) holds for the plane $\tilde{u}_{0}$. Let $P=(x, T) \in \mathcal{P}(\lambda)$ satisfying $v_{n}\left(B_{R_{n}}(0)\right) \cap x(\mathbb{R})=\emptyset, \forall n$. Consider the finite energy immersion

$$
F: \mathbb{C} \backslash\{0\} \rightarrow \mathbb{R} \times S^{3}, \quad \zeta \mapsto\left(\frac{T \log |\zeta|}{2 \pi}, x\left(\frac{T \arg \zeta}{2 \pi}\right)\right)
$$

and define

$$
A=\left\{(z, \zeta) \in \mathbb{C} \times(\mathbb{C} \backslash 0) \mid \tilde{u}_{0}(z)=F(\zeta)\right\} .
$$

If the set $A$ is not empty, it consists of isolated points. Indeed, assume that $\left(z^{*}, \zeta^{*}\right)$ is not an isolated point of $A$. Since both $\tilde{u}_{0}$ and $F$ are immersions, we can use Lemma 2.4.3 of MS04 to find open neighborhoods $\mathcal{O}$ and $\mathcal{O}^{\prime}$ of $z^{*}$ and $\zeta^{*}$ respectively, and a holomorphic diffeomorphism $f: \mathcal{O} \rightarrow \mathcal{O}^{\prime}$ such that $F \circ f=\tilde{u}_{0}$ on $\mathcal{O}$. Since $u_{0}$ is transverse to the Reeb flow, we get a contradiction. Now assume $A \neq \emptyset$ and choose $\left(z^{*}, \zeta^{*}\right) \in A$. The maps $\tilde{u}_{0}$ and $F$ intersect transversally at the pair $\left(z^{*}, \zeta^{*}\right)$. By positivity and stability of intersections of pseudo-holomorphic immersions, (see Theorem 2.6.3 and exercise 2.6.7 of [MS04]) we find $z_{j} \rightarrow z^{*}$ and $n_{j} \rightarrow \infty$ such that $\tilde{v}_{n_{j}}\left(z_{j}\right) \in F(\mathbb{C} \backslash\{0\})$. This contradicts the fact that $v_{n}(\mathbb{C}) \cap x(\mathbb{R})=\emptyset, \forall j$. So we have proved that $A=\emptyset$ and consequently $u_{0}(\mathbb{C} \backslash\{0\}) \cap P=\emptyset$.

If, in addition, $P=(x, T)$ is simply covered and $\mu(P) \geq 3$, then it follows from the fact that $\mu\left(P_{0}\right)=2$ and item (4) in Lemma 1.9 that $P$ and $P_{0}$ are geometrically distinct. This proves (3).

\subsection{Transverse foliations}

To state the main theorem of this Thesis, which will be done in Chapter 3 , we need the concept of transverse foliation adapted to a flow. This is a refined version of the concept of global systems of transverse sections introduced in HWZ03.

Definition 2.40 (Transverse Foliation). [HSa18 Let $M$ be a closed oriented 3 -manifold and let $\phi^{t}$ be a flow on $M$. A transverse foliation for $\phi^{t}$ consists of

- A finite set $\mathcal{P}$ of simple periodic orbits of $\phi^{t}$, called binding orbits;

- A smooth foliation of $M \backslash \cup_{P \in \mathcal{P}} P$ by properly embedded surfaces. Every leaf is transverse to $\phi^{t}$ and has an orientation induced by $\phi^{t}$ and $M$. 
For every leaf $\dot{\Sigma}$ there exists a compact embedded surface $\Sigma \hookrightarrow M$ so that $\dot{\Sigma}=\Sigma \backslash \partial \Sigma$ and $\partial \Sigma$ is a union of connected components of $\cup_{P \in \mathcal{P}} P$. An end $z$ of $\dot{\Sigma}$ is called a puncture. To each puncture $z$ there is an associated component $P_{z} \in \mathcal{P}$ of $\partial \Sigma$, called the asymptotic limit of $\dot{\Sigma}$ at $z$. A puncture $z$ of $\dot{\Sigma}$ is called positive if the orientation on $P_{z}$ induced by $\Sigma$ coincides with the orientation induced by $\phi^{t}$. Otherwise $z$ is called negative.

Transverse foliations adapted to Reeb flows on $S^{3}$ can be obtained as the projection onto $S^{3}$ of a stable finite energy foliation in the symplectization $\mathbb{R} \times S^{3}$. See Definition 2.41 below.

\subsubsection{Finite energy foliations}

Consider $S^{3}$ equipped with a nondegenerate tight contact form $\lambda=$ $f \lambda_{0} \mid S^{3}$.

Definition 2.41. [HWZ03] A stable finite energy foliation for $\left(S^{3}, \lambda, J\right)$ is a smooth foliation $\mathcal{F}$ of $\mathbb{R} \times S^{3}$ with the following properties.

- There exists a uniform constant $C>0$ such that for every leaf $F \in \mathcal{F}$, there exists an embedded finite energy $\tilde{J}$-holomorphic sphere $\tilde{u}: S^{2} \backslash$ $\Gamma \rightarrow \mathbb{R} \times S^{3}$ satisfying

$$
F=\tilde{u}\left(S^{2} \backslash \Gamma\right) \quad \text { and } \quad E(\tilde{u}) \leq C
$$

- The translation

$$
T_{r}(F)=r+F:=\{(r+a, m) \mid(a, m) \in F\}, F \in \mathcal{F}, r \in \mathbb{R}
$$

defines an $\mathbb{R}$-action on $\mathcal{F}$;

- The asymptotic limits of a leaf $F=\tilde{u}\left(S^{2} \backslash \Gamma\right) \in \mathcal{F}$ are defined to be the asymptotic limits of $\tilde{u}$ at its punctures. For every leaf $F$, the asymptotic limits are simply covered, their Conley-Zehnder indices are contained in $\{1,2,3\}$ and their self-linking numbers are equal to -1 ;

- If $F=\tilde{u}\left(S^{2} \backslash \Gamma\right) \in \mathcal{F}$, then $\tilde{u}$ has precisely one positive puncture, but an arbitrary number of negative punctures. We define $\operatorname{ind}(F):=\operatorname{ind}(\tilde{u})$. The index does not depend on the choice of $\tilde{u}$. If $F$ is not a fixed point of the $\mathbb{R}$-action, then $\operatorname{ind}(F) \in\{1,2\}$. 
Consider a leaf $F \in \mathcal{F}$ that is not a fixed point of the $\mathbb{R}$-action. Write $\Gamma=\Gamma^{+} \cup \Gamma^{-}=\Gamma^{+} \cup \Gamma_{1}^{-} \cup \Gamma_{2}^{-} \cup \Gamma_{3}^{-}$, where $\Gamma_{j}^{-}$is the set of punctures having Conley-Zehnder index $j$. Note that by definition $\Gamma^{+}=1$. Denote by $\mu^{+}$the Conley-Zehnder index of the unique positive puncture. Then, by formula (2.9) for the Fredholm index,

$$
\text { ind } \begin{aligned}
F & =\mu^{+}-3 \# \Gamma_{3}^{-}-2 \# \Gamma_{2}^{-}-\# \Gamma_{1}^{-}-2+1+\# \Gamma_{1}^{-}+\# \Gamma_{2}^{-}+\# \Gamma_{3}^{-} \\
& =\mu^{+}-1-2 \# \Gamma_{3}^{-}-\# \Gamma_{2}^{-}
\end{aligned}
$$

Since, by definition, $\operatorname{ind}(F) \geq 1$, we get

$$
2 \# \Gamma_{3}^{-}+\# \Gamma_{2}^{-} \leq \mu^{+}-2 .
$$

Using $\mu^{+} \in\{2,3\}$, we conclude

$$
\mu^{+} \in\{2,3\}, \quad \# \Gamma_{3}^{-}=0 \quad \text { and } \# \Gamma_{2}^{-} \leq 1 .
$$

Note that there is no restriction on $\# \Gamma_{1}^{-}$.

Therefore, a leaf $F \in \mathcal{F}$ which is not a fixed point of the $\mathbb{R}$-action satisfies one of the following alternatives.

- $\mu^{+}=3, \# \Gamma_{2}^{-}=0$ and ind $F=2$.

- $\mu^{+}=3, \# \Gamma_{2}^{-}=1$ and $\operatorname{ind}(F)=1$.

- $\mu^{+}=2, \# \Gamma_{2}^{-}=0$ and $\operatorname{ind}(F)=1$.

In any case, the number of negative punctures with Conley-Zehnder index 1 is arbitrary.

By definition, the energies $E(\tilde{u})$ are uniformly bounded. Since the periods of the asymptotic limits are bounded by the energy and $\lambda$ is nondegenerate, the number of all asymptotic limits appearing in $\mathcal{F}$ is finite.

The existence of stable finite energy foliations for generic almost complex structures $\tilde{J}$ was proved in [HWZ03.

Theorem 2.42 (Hofer-Wysocki-Zehnder). Let $\lambda$ be a nondegenerate tight contact form on $S^{3}$. There exists a residual subset of $\mathcal{J}(\xi, d \lambda)$ in the $C^{\infty}$ topology such that there exists a stable finite energy foliation for $\left(S^{3}, \lambda, J\right)$ containing a finite energy plane and satisfying the following properties:

- The fixed points of the $\mathbb{R}$-action on $\mathcal{F}$ are orbit cylinders;

- Let $p: \mathbb{R} \times S^{3} \rightarrow S^{3}$ be the projection onto the second factor. If two leaves $F$ and $G$ do not belong to the same orbit of the action, then $p(F) \cap p(G)=\emptyset$; 
- If $F \in \mathcal{F}$ is not a fixed point of the $\mathbb{R}$-action, then $p(F)$ is an embedded punctured sphere in $S^{3}$ which is transverse to the Reeb flow and coverges at the punctures to the asymptotic limits of $F$.

- Denote by $\mathcal{P}$ the set of asymptotic limits of $\mathcal{F}$. Then $p(\mathcal{F})$ is a singular foliation of $S^{3}$ having $\mathcal{P}$ as the singular set.

It follows from the Fredholm theory of [HWZ99b] that a leaf satisfying $\operatorname{ind}(F)=2$ belongs to a 2-parameter family of leaves, all having the same asymptotic limits, where one parameter is defined by the $\mathbb{R}$-action on $\mathcal{F}$. The image of the 2-parameter family under the projection $p: \mathbb{R} \times S^{3} \rightarrow S^{3}$ is a 1-parameter family of embedded punctured spheres.

A leaf satisfying $\operatorname{ind}(F)=1$ belongs to a 1-parameter family defined by the $\mathbb{R}$-action. The projection of this family onto $S^{3}$ is an isolated embedded punctured sphere, that is called a rigid surface.

The existence of a transverse foliation adapted to any nondegenerate Reeb flow on $\left(S^{3}, \xi_{0}\right)$, where $\xi_{0}$ is the tight contact structure on $S^{3}$, is a corollary of Theorem 2.42. We state this corolary below.

Theorem 2.43 ([HWZ03]). Let $\phi^{t}$ be a nondegenerate Reeb flow on $\left(S^{3}, \xi_{0}\right)$. Then $\phi^{t}$ admits a transverse foliation. The binding orbits have self-linking number -1 and Conley-Zehnder indices in $\{1,2,3\}$. Every leaf $\dot{\Sigma}$ is a punctured sphere and has precisely one positive puncture. One of the following conditions holds:

- The asymptotic limit of $\dot{\Sigma}$ at its positive puncture has Conley-Zehnder index 3 and the asymptotic limit of $\dot{\Sigma}$ at any negative puncture has Conley-Zehnder index 1 or 2. There exists at most one negative puncture whose asymptotic limit has Conley-Zehnder index 2.

- The asymptotic limit of $\dot{\Sigma}$ at its positive puncture has Conley-Zehnder index 2 and the asymptotic limit of $\dot{\Sigma}$ at any negative puncture has Conley-Zehnder index 1.

\subsubsection{Open book decompositions}

Assume that the foliation $\mathcal{F}$ given by Theorem 2.42 has precisely one fixed point of the $\mathbb{R}$-action, that is, there is precisely one asymptotic limit $P$ in $\mathcal{P}$. Then $\mu(P)=3$ and $\mathcal{F}$ consists of a cylinder over the orbit $P$ and a family of pseudoholomorphic planes asymptotic to $P$. The projection of $\mathcal{F}$ onto $S^{3}$ is an open book decomposition ${ }^{3}$ with pages of disk type, all having

\footnotetext{
${ }^{3}$ An open book decomposition of a 3 -manifold $M$ is a pair $(L, p)$ where $L \subset M$ is a link and $p: M \backslash L \rightarrow S^{1}$ is a fibration such that each fiber $p^{-1}(\theta)$ is the interior of a compact
} 


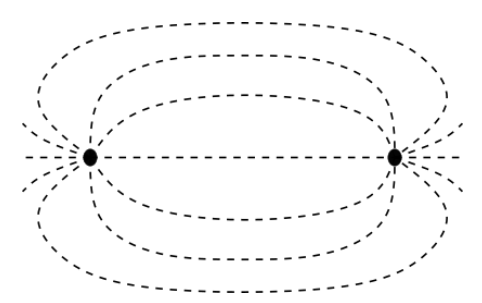

Figure 2.1: An open book decomposition cut by a plane. The dots represent a periodic orbit with Conley-Zehnder index 3. The dashed curves represent a family of planes with boundary $P$. The 3 -sphere is viewed as $\mathbb{R}^{3} \cup\{\infty\}$.

the orbit $P$ as boundary. By the arguments in [HWZ98, one can show that the regular leaves of $p(\mathcal{F})$ are global surfaces of section for the Reeb flow associated with $\lambda$. Moreover, the first return map is conjugated to an areapreserving diffeomorphism of the open unit disk. Since the area of the disk is finite, Brouwer's translation theorem gives a periodic point of the first return map. It is a initial condition to a periodic solution of the Reeb vector field which is different from $P$. If the return map has a second periodic point, then by a Theorem of J. Franks [Fra92] on area-preserving homeomorphisms of the open annulus, the return map has infinitely many periodic points, so that $X_{\lambda}$ has infinitely many periodic orbits. This is the case of dynamically convex contact forms.

Definition 2.44. A contact form $\lambda$ on $S^{3}$ is called dynamically convex if $\mu(P) \geq 3$ for all periodic solution $P$ of the associated Reeb vector field $X_{\lambda}$.

If the contact form $\lambda$ is nondegenerate and dynamically convex, then the finite energy foliation given by Theorem 2.42 has precisely one fixed point of the $\mathbb{R}$-action, and we conclude the following corollary of Theorem 2.42 .

Corollary 2.45. The Reeb vector field $X_{\lambda}$ associated with a nondegenerate and dynamically convex contact form $\lambda=f \lambda_{0}$ on $S^{3}$ possesses a global surface of section. Further, there are either 2 or infinitely many periodic orbits of $X_{\lambda}$.

It is proved in [HWZ98] that the statement above holds true without the nondegeneracy condition on $\lambda$.

embedded surface $S_{\theta} \hookrightarrow M$ satisfying $\partial S_{\theta}=L . L$ is called the binding and the fibers are called pages. If the pages are disks we say that $(L, p)$ has disk-like pages. 


\section{Chapter 3}

\section{3-2-1 Foliations and the main theorem}

In this chapter, we define $3-2-1$ foliations, state the main theorem of this thesis and give a sketch of its proof.

\subsection{Main Theorem}

Let $\lambda$ be a nondegenerate tight contact form on $S^{3}$. Let $R_{\lambda}$ be the associated Reeb vector field and denote by $\varphi^{t}$ the flow of $R_{\lambda}$.

Before defining $3-2-1$ foliations, we need the definition of strong transverse sections.

Definition 3.1 (Strong transverse section). Let $\Sigma \hookrightarrow S^{3}$ be a compact embedded surface such that $\dot{\Sigma}=\Sigma \backslash \partial \Sigma$ is transverse to $\varphi^{t}$ and $\partial \Sigma$ consists of a finite number of simple orbits in $\mathcal{P}(\lambda)$. $\Sigma$ is called a strong transverse section if every orbit $P_{z}=(x, T)$ associated to an end $z$ of $\dot{\Sigma}$ has a neighborhood on $\Sigma$ parametrized by $\psi:\left(r_{0}, 1\right] \times \mathbb{R} / \mathbb{Z} \rightarrow \Sigma$, such that $\psi(1, t)=x_{T}(t)$, $\forall t \in \mathbb{R} \times \mathbb{Z}$, and the section of $x_{T}^{*} \xi$ defined by

$$
\eta(t)=\left.\frac{\partial}{\partial r} \psi(r, t)\right|_{r=1}
$$

satisfies

$$
d \lambda\left(\eta(t), \mathcal{L}_{R} \eta(t)\right) \neq 0, \forall t \in \mathbb{R} \times \mathbb{Z},
$$

where $\mathcal{L}_{R}$ is the Lie derivative in the direction of $R_{\lambda}$ as defined in (1.6).

Remark 3.2. Any transverse section given by the projection of a $\tilde{J}$-holomorphic curve satisfies the definition above. Indeed, assume $\dot{\Sigma}=u(\dot{S})$, where $\tilde{u}=$ 
$(a, u): \dot{S} \rightarrow \mathbb{R} \times S^{3}$ is a finite energy $\tilde{J}$-holomorphic curve. Then, by Theorem 2.11, for every puncture $z$ with asymptotic limit $P$, the section $\pi_{\xi} \eta$, where $\eta$ is defined as in Definition 3.1, is an eigensection of the asymptotic operator $A_{P, J}$ associated with a nonzero eigenvalue $c$ and $\eta(t) \neq 0, \forall t \in S^{1}$. Thus, by the definition of $A_{P, J}$ in (1.8) and equation (1.7), we have

$$
d \lambda\left(\eta(t), \mathcal{L}_{R} \eta(t)\right)=\frac{1}{T} d \lambda\left(\eta(t), J A_{P, J} \eta(t)\right)=\frac{1}{T} c d \lambda(\eta(t), J \eta(t)) \neq 0, \forall t .
$$

Remark 3.3. In what follows we fix a global unitary trivialization

$$
\Psi: \xi_{0} \rightarrow S^{3} \times \mathbb{R}^{2}
$$

of the tight contact structure $\xi_{0}=\operatorname{ker} \lambda$ on $S^{3}$. All indices are computed with respect to this fixed trivialization. Recall that in $\left(S^{3}, \xi_{0}\right)$ the Conley-Zehnder index and the index wind ${ }_{\infty}$ do not depend on the chosen global trivialization.

Definition 3.4 (3-2-1 Foliation). A 3-2-1 foliation for the Reeb flow $\varphi^{t}$ on $\left(S^{3}, \xi_{0}=\operatorname{ker} \lambda\right)$ is a transverse foliation for $\varphi^{t}$ satisfying the following properties: The set $\mathcal{P}$ of binding orbits consists of three prime orbits $P_{1}, P_{2}$ and $P_{3}$ with Conley-Zehnder indices respectively 1, 2 and 3. $P_{1} \cup P_{2} \cup P_{3}$ is a unlink. The foliation of $S^{3} \backslash \cup_{P \in \mathcal{P}} P$ consists of

- A pair of cylinders $V_{1}$ and $V_{2}$, asymptotic to $P_{3}$ at their positive punctures and to $P_{2}$ at their negative punctures. $T:=V_{1} \cup V_{2} \cup P_{2} \cup P_{3}$ is homeomorphic to a torus and $T \backslash x_{3}(\mathbb{R})$ is $C^{1}$-embedded. $T$ divides $S^{3}$ into two closed regions $\mathcal{R}_{1}$ and $\mathcal{R}_{2}$ with boundary $T$.

- A disk $D \subset \mathcal{R}_{1}$ asymptotic to $P_{2}$ at its positive puncture;

- A cylinder $U \subset \mathcal{R}_{2}$ asymptotic to $P_{2}$ at its positive puncture and to $P_{1}$ at its negative puncture;

- $D \cup U$ is a $C^{1}$-embedded disk with boundary $P_{1}$ and transverse to $T$;

- A 1-parameter family $F_{\tau} \subset \mathcal{R}_{1}$ of planes asymptotic to $P_{3}$ at its positive puncture;

- A 1-parameter family of cylinders $C_{\tau} \subset \mathcal{R}_{2}$ asymptotic to $P_{3}$ at its positive puncture and to $P_{1}$ at its negative puncture;

- Every regular leaf of the foliation is a strong transverse section.

See figure 3.1 . 


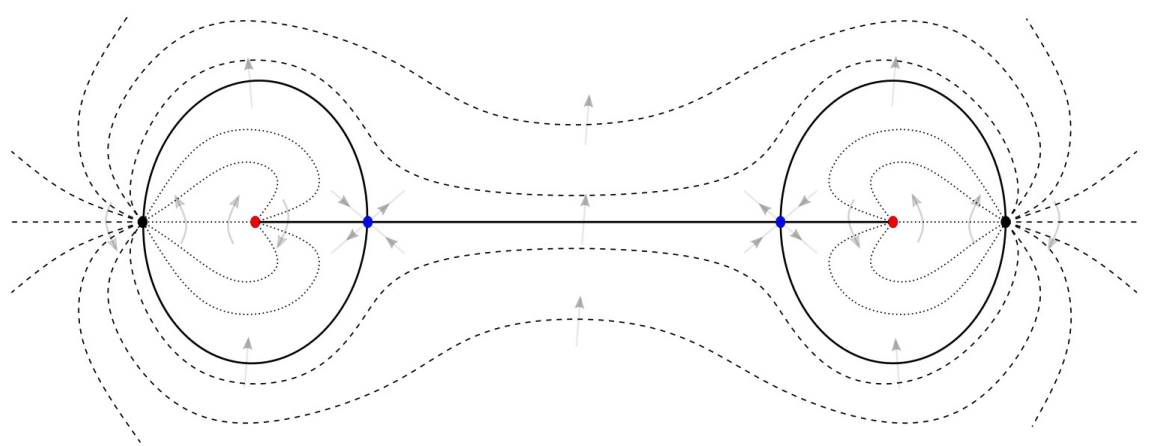

Figure 3.1: A $3-2-1$ foliation cut by a plane. The dots represent periodic orbits $P_{1}, P_{2}$ and $P_{3}$ with Conley-Zehnder indices respectively 1,2 and 3 . The bold curves represent two rigid cylinders connecting $P_{2}$ and $P_{3}$, a rigid cylinder connecting $P_{1}$ and $P_{2}$, and a rigid disk with boundary $P_{2}$. The dashed curves represent a family of planes with boundary $P_{3}$. The dotted curves represent a family of cylinders connecting $P_{1}$ and $P_{3}$. The arrows indicate the Reeb flow. The 3 -sphere is viewed as $\mathbb{R}^{3} \cup\{\infty\}$.

Now we state the main theorem of this thesis.

Theorem 3.5. Let $\lambda$ be a nondegenerate tight contact form on $S^{3}$. Let $P_{1}=\left(x_{1}, T_{1}\right), P_{2}=\left(x_{2}, T_{2}\right), P_{3}=\left(x_{3}, T_{3}\right) \in \mathcal{P}(\lambda)$ be simply covered closed Reeb orbits with Conley-Zehnder indices respectively 1, 2 and 3. Assume that the orbits $P_{1}, P_{2}$ and $P_{3}$ are unknotted, $P_{i}$ and $P_{j}$ are not linked for $i \neq j$, $i, j \in\{1,2,3\}$ and the following conditions hold:

(i) $P_{3}$ spans an embedded disk whose interior is transverse to the Reeb flow;

(ii) $T_{1}<T_{2}<T_{3}<2 T_{1}$;

(iii) $P_{2}$ is the only Reeb orbit with Conley-Zehnder index 2 not linked to $P_{3}$ with period $<T_{3}$;

(iv) $P_{1}$ is the only Reeb orbit with Conley-Zehnder index 1 not linked to $P_{2}$ with period $<T_{2}$;

(v) There is no $C^{1}$-embedding $\Psi: S^{2} \rightarrow S^{3}$ such that $x_{2}\left(T_{2} \cdot\right)=\left.\Psi\right|_{S^{1} \times\{0\}}$ and each hemisphere is a strong transverse section.

Then $P_{1}, P_{2}$ and $P_{3}$ are the binding orbits of a $3-2-1$ foliation.

Condition $(v)$ in Theorem 3.5 is also necessary. This is the content of Proposition 3.6 below. 
Proposition 3.6. Assume there exists a 3-2-1 foliation for the Reeb flow $\varphi^{t}$ and let $P_{2}=\left(x_{2}, T_{2}\right)$ be the binding orbit with Conley-Zehnder index 2, as in Definition 3.4. Then there is no $C^{1}$-embedding $\psi: S^{2} \rightarrow S^{3}$ such that $x_{2}\left(T_{2} \cdot\right)=\left.\psi\right|_{S^{1} \times\{0\}}$ and each hemisphere is a strong transverse section.

\subsubsection{Sketch of the proof of Theorem 3.5}

Following [Hof93, HWZ95a, HWZ99a, HSa11], we consider a Bishop family of disks with boundary on a suitable disk $\mathcal{D}$ spanning $P_{3}$. By hypothesis there is an orbit with Conley-Zehnder index 2 not linked to $P_{3}$. So, we have to consider the possibility that the Bishop family breaks before it approaches $\partial \mathcal{D}$. We divide our analysis into two cases:

- Either the boundaries of the disks in the Bishop family tend to $P_{3}$, or

- The Bishop family breaks before it approaches $\partial \mathcal{D}$.

In the first case, the Bishop family produces either a plane asymptotic to $P_{3}$, so that we have a family of such planes, or a rigid cylinder asymptotic to $P_{3}$ and to $P_{2}$ and a rigid plane asymptotic to $P_{2}$, so that we can apply the gluing theorem to produce a family of planes asymptotic to $P_{3}$. Using our assumptions, we prove that the family of planes is noncompact. The closure of the family of projected planes is a solid torus $\mathcal{R}_{1} \subset S^{3}$. The family of planes, together with a pair of rigid cylinders and a rigid plane to $P_{2}$ form part of the 3-2-1 foliation. By the Fredholm theory of [HWZ99b], the plane asymptotic to $P_{2}$ lies in a one dimensional family of such planes in a symplectic cobordism. We show that the family is non compact, and produces a rigid cylinder asymptotic to $P_{2}$ and $P_{1}$. Applying the gluing theorem again, we produce a 1-parameter family of cylinders asymptotic to the orbits $P_{3}$ and $P_{1}$. This completes the $3-2-1$ foliation.

We do not give a complete proof to the second case in this thesis, however, we present the main ideas of the proof in Chapter 5. In the second case, we just obtain a rigid plane to $P_{2}$ from the Bishop family. As in the first case, using the rigid plane to $P_{2}$, we can prove the existence of a rigid cylinder connecting $P_{2}$ and $P_{1}$. Using gluing, we can obtain a family of pesudoholomorphic half-cylinders with boundary in $\mathcal{D}$ and a negative puncture asymptotic to $P_{1}$. We show that the boundaries of the half-cylinders in this family "go in the direction of the boundary of $\mathcal{D}$ ". If this family breaks before it approaches the boundary, we use gluing theorem again to produce a family of disks with boundary in $\mathcal{D}$. We repeat this process until we obtain a family of disks or half-cylinders whose boundaries converge to $\partial \mathcal{D}$. After this step we produce either a cylinder connecting $P_{3}$ and $P_{1}$ or a plane to $P_{3}$. From this point on, the analysis is basically the same as in the first case. 


\subsection{Proof of Proposition 3.6}

In this section we use the notation of Definition 3.4.

Lemma 3.7. If there exists a $C^{1}$-embedding $\psi: S^{2} \rightarrow S^{3}$ such that $x_{2}\left(T_{2} \cdot\right)=$ $\left.\psi\right|_{S^{1} \times\{0\}}$ and $\psi\left(S^{2}\right) \backslash x_{2}(\mathbb{R})$ is transverse to the Reeb flow, then we can assume that $\left.\psi\right|_{S^{2} \backslash S^{1} \times\{0\}}$ is also transverse to $T \backslash\left(x_{3}(\mathbb{R}) \cup x_{2}(\mathbb{R})\right)=V_{1} \cup V_{2}$.

Proof. Define

$$
\begin{aligned}
F: \mathbb{R} \times S^{2} & \rightarrow S^{3} \\
(t, x) & \mapsto \varphi^{t} \circ \psi(x) .
\end{aligned}
$$

Then $F$ satisfies $F(t, \cdot)\left(S^{1} \times\{0\}\right)=\psi\left(S^{1} \times\{0\}\right), \forall t \in \mathbb{R}$, and

$$
d F_{(t, x)}(a, v)=a R\left(\varphi^{t} \circ \psi(x)\right)+d \varphi_{\psi(x)}^{t} \cdot d \psi_{x} v
$$

for every $(t, x) \in \mathbb{R} \times S^{2}$ and $(a, v) \in \mathbb{R} \times T_{x} S^{2}$. We claim that for every $x \in S^{2} \backslash S^{1} \times\{0\}$ and $t \in \mathbb{R}, d F(t, x)$ is surjective. Since $\psi$ is transverse to the Reeb flow away from $S^{1} \times\{0\}$, we know that $\pi_{\xi} \circ d \psi_{x}$ is surjective. Since $d \varphi^{t}$ preserves the splitting $T S^{3}=\mathbb{R} R \oplus \xi$, it follows that $\pi_{\xi} \circ d \varphi_{\psi(x)}^{t} \circ d \psi_{x}$ is surjective. We conclude that $d F(t, x)$ is surjective for all $x \in S^{2} \backslash S^{1} \times\{0\}$ and $t \in \mathbb{R}$. In particular, $F$ is transverse to $T \backslash x_{3}(\mathbb{R})$ on $\mathbb{R} \times\left(S^{2} \backslash S^{1} \times\{0\}\right)$. This implies ${ }^{1}$ that for almost all $t \in \mathbb{R},\left.F(t, \cdot)\right|_{S^{2} \backslash S^{1} \times\{0\}}$ is transverse to $T$.

Also, $F(t, \cdot)^{*} d \lambda=\left(\varphi^{t} \circ \psi\right)^{*} d \lambda=\psi^{*} d \lambda$, and it follows that $F(t, \cdot)$ is still transverse to the flow for all $t \in \mathbb{R}$. Thus, we can replace $\psi$ with $F(t, \cdot)$ for some $t$ such that $\left.F(t, \cdot)\right|_{S^{2} \backslash S^{1} \times\{0\}} \pitchfork T$ to get the desired embedding.

Lemma 3.9. Assume that $\psi: S^{2} \rightarrow S^{3}$ is a $C^{1}$ embedding such that $x_{2}\left(T_{2} \cdot\right)=\left.\psi\right|_{S^{1} \times\{0\}}$ and such that each hemisphere is a strong transverse section. Then $\psi$ is transverse to the torus $T$ along $P_{2}$.

The proof of Lemma 3.9 is given in Appendix A.

Proof of Proposition 3.6. Suppose, by contradiction, that $\psi: S^{2} \rightarrow S^{3}$ is a $C^{1}$ embedding such that $x_{2}\left(T_{2} \cdot\right)=\left.\psi\right|_{S^{1} \times\{0\}}$ and such that each hemisphere is a strong transverse section.

Let $T=V_{1} \cup V_{2} \cup P_{2} \cup P_{3}$ be the torus given by Definition 3.4. $T$ divides $S^{3}$ into two connected regions $\mathcal{R}_{1}$ e $\mathcal{R}_{2}$. The region $\mathcal{R}_{1}$ contains an

\footnotetext{
${ }^{1}$ Applying the following theorem to $F$ restricted to $S^{2} \backslash\left(S^{1} \times\{0\}\right)$ :

Theorem 3.8 (GP10). Let $X, S$ and $Y$ be smooth manifolds, $Z$ submanifold of $Y$ and $F: X \times S \rightarrow Y$ smooth. If $F$ is transverse to $Z$, then for almost all $s \in S, f_{s}:=F(s, \cdot)$ is transverse to $Z$.
} 
embedded disk with boundary $x_{2}(\mathbb{R})$, so that $P_{2}$ is contractible in $\mathcal{R}_{1}$. One can also show, using Mayer-Vietoris sequence, that the holomology class of $x_{T_{2}}$ generates $H_{1}\left(\mathcal{R}_{2}, \mathbb{Z}\right)$.

Since, by Lemma 3.9, $\psi$ is transverse to the torus $T$ along $P_{2}$, we know that the image of any neighborhood of $S^{1} \times\{0\} \subset S^{2}$ by $\psi$ intersects both $\mathcal{R}_{1}$ and $\mathcal{R}_{2}$ away from $\psi\left(S^{1} \times\{0\}\right)$. This implies that the sphere $\psi\left(S^{2}\right)$ intersects the torus $T$ away from $x_{2}(\mathbb{R})$. Indeed, one of the hemispheres of $\psi\left(S^{2}\right)$ intersects $\mathcal{R}_{2}$ and can not be contained in $\mathcal{R}_{2}$, because this would imply $P_{2}$ contractible in $\mathcal{R}_{2}$, which is a contradiction since the holomology class of $x_{T_{2}}$ generates $H_{1}\left(\mathcal{R}_{2}, \mathbb{Z}\right)$.

By Definition 3.4, the orbits $P_{3}$ and $P_{2}$ are not linked, that is, the linking number $\operatorname{lk}\left(P_{2}, P_{3}\right)$ is zero. Note that the image of each closed hemisphere of $S^{2}$ by $\psi$ is a Seifert surface for $P_{2}$, which is transverse to the Reeb flow. Then $\operatorname{lk}\left(P_{2}, P_{3}\right)$ is the intersection number of $P_{3}$ and $\psi$ restricted to one of the hemispheres of $S^{2}$. Since $\operatorname{lk}\left(P_{2}, P_{3}\right)=0$ and $\left.\psi\right|_{S^{2} \backslash S^{1} \times\{0\}}$ is transverse to $P_{3}$, we conclude that $\psi\left(S^{2}\right) \cap P_{3}=\emptyset$. By Lemmas 3.7 and 3.9, we know that $\psi \pitchfork T \backslash P_{3}$. Thus, $\psi$ intersects $T$ transversely and the intersection $\psi\left(S^{2}\right) \cap T$ is contained in a closed subset of $T \backslash P_{3}$. We conclude that the preimage of the intersection $\psi\left(S^{2}\right) \cap T$ by $\psi$ is a 1-dimensional submanifold of $S^{2}$ which is a closed subset of $S^{2}$. It follows that each connected component of $\psi^{-1}\left(\psi\left(S^{2}\right) \cap T\right)$ is diffeomorphic to $S^{1}$.

$S^{1} \times\{0\}$ is one of the connected components of the boundary of a region $R \subset S^{2}$ such that $\psi(R) \subset \mathcal{R}_{2}$. Thus, one of the other connected components of the boundary of $R$, that we denote by $S$, is such that $\left.\psi\right|_{S}$ is homologous to $x_{2 T_{2}}$ in $\mathcal{R}_{2}$. Denoting $H^{1}(T, \mathbb{Z})=\mathbb{Z}\left[x_{2 T_{2}}\right] \oplus \mathbb{Z}[m]$, the homology class of $\left.\psi\right|_{S}$ in $H^{1}(T, \mathbb{Z})$ is $(1, l)$ for some $l \in \mathbb{Z}$. Since $S$ does not intersect $x_{2}(\mathbb{R}), l$ must be zero, that is, the class of $\left.\psi\right|_{S}$ is $(1,0)$. This implies that $\psi(S)$ and $x_{2}(\mathbb{R})$ divide $T$ into two connected regions with boundary $x_{2}(\mathbb{R}) \cup \psi(S)$.

Now fix an orientation on $S^{1} \times\{0\} \subset S^{2}$ in such a way that $\left.\psi\right|_{S^{1} \times\{0\}}$ preserves orientation. Consider the closed hemispheres of $S^{2}$, that we call $H_{+}$e $H_{-}$, with the orientation induced by the orientation of $S^{1} \times\{0\}$. It follows that

$$
0<T_{2}=\int_{S^{1}} x_{2}^{*} T_{2} \lambda=\int_{H_{ \pm}} \psi^{*} d \lambda
$$

Since $\psi$ is transverse to the Reeb flow $R_{\lambda}$ in $H_{ \pm} \backslash S^{1} \times\{0\}$, then $\psi^{*} d \lambda>0$ in $H_{ \pm} \backslash S^{1} \times\{0\}$. Let $B$ be the connected region of $S^{2}$ bounded by $S^{1} \times\{0\}$ and $S$. Since $B$ is contained in one of the hemispheres of $S^{2}$, then

$$
0<\int_{B} \psi^{*} d \lambda=\int_{S^{1}} x_{2 T_{2}}^{*} \lambda-\int_{S} \psi^{*} \lambda=T_{2}-\int_{\psi(S)} \lambda
$$


Since $\psi\left(S^{2}\right)$ does not intersect $P_{3}$, we know that one of the regions of $T$ bounded by $P_{2}$ and $\psi(S)$, that we denote by $A$, satisfies either $A \subset V_{1}$ or $A \subset V_{2}$. Recall that $V_{1}$ and $V_{2}$ are oriented in such a way that $d \lambda \mid V_{1,2}$ is an area form, $P_{3}$ is a positive asymptotic limit and $P_{2}$ is a negative asymptotic limit. Then we have

$$
0<\int_{A} d \lambda=\int_{\psi(S)} \lambda-\int_{x_{2}(\mathbb{R})} \lambda=\int_{\psi(S)} \lambda-T_{2} .
$$

Leading to a contradiction.

\subsection{Proof of the main theorem}

In this section we present the first steps in the proof of Theorem 3.5 .

Consider $S^{3}$ equipped with a nondegenerate tight contact form $\lambda$. Let $P_{1}$, $P_{2}$ and $P_{3}$ be closed Reeb orbits satisfying the hypotheses of Theorem 3.5 .

\subsubsection{A special spanning disk for the orbit $P_{3}$}

Let $F$ be a closed disk embedded in $S^{3}$ such that $\partial F$ is transverse to the contact structure $\xi=\operatorname{ker} \lambda$. The contact structure induces a singular distribution

$$
(\xi \cap T F)^{d \lambda}
$$

on $F$, called the characteristic distribution of $F$. Observe that

$$
\begin{gathered}
T_{p} F=\left.\xi\right|_{p} \Rightarrow\left(\left.\xi\right|_{p} \cap T_{p} F\right)^{d \lambda}=0 \\
\left.\left.T_{p} F \cap \xi\right|_{p} \subsetneq \xi\right|_{p} \Rightarrow\left(\left.\xi\right|_{p} \cap T_{p} F\right)^{d \lambda}=\left.\xi\right|_{p} \cap T_{p} F .
\end{gathered}
$$

The characteristic foliation can be parametrized by a smooth vector field $V$ on $\mathrm{F}$ in a canonical way, see [HWZ95b, Section 4] for details. In other words, $V_{p}=0$ if and only if $T_{p} F=\xi_{p}$ and $\mathbb{R} V_{p}=T_{p} F \cap \xi_{p}$ if and only if $T_{p} F \cap \xi_{p} \varsubsetneqq \xi_{p}$. Moreover, we can assume that $V$ points outwards at the boundary $\partial F$.

Let $p \in F$ be a nondegenerate zero of $V$, that is, the linearization $d V_{p}$ is an isomorphism of $\xi_{p}=T_{p} F$. If $a$ and $b$ are the eigenvalues of $d V_{p}$, then $a b \neq 0$. The point $p$ is called elliptic if $a b>0$ and hyperbolic if $a b<0$. An elliptic point is called nicely elliptic if $a$ and $b$ are real numbers.

Denote by $o$ the orientation of $F$ so that the induced orientation on the boundary satisfies $\left.\lambda\right|_{T \partial F}>0$. We also have a fiberwise orientation $o^{\prime}$ of $\left.\xi\right|_{F}$ induced by $d \lambda$. A nondegenerate zero $p$ of $V$, where by definition $T_{p} F=\xi_{p}$, 
is positive if $o$ coincides with $o^{\prime}$ and negative otherwise. Negative elliptic points are sinks and positive elliptic points are sources for the vector field $V$, see HWZ95b for details.

The following theorem is a particular case of [HWZ95a, Theorem 3.2].

Theorem 3.10 ([HWZ95a]). Let $\lambda$ be a contact form on $S^{3}$ with associated contact structure $\xi$ and Reeb vector field $R_{\lambda}$. Assume there exists a nondegenerate and simply covered orbit $P=(x, T)$ satisfying $\mu(P)=3$, which spans an embedded disk $F_{0}$ whose interior is transverse to $R_{\lambda}$. Then there exists an embedded disk $F$ spanning $P$ whose interior is transverse to $R$ and whose characteristic foliation is transverse to $\partial F$ and possesses precisely one singular point $e$, which is positive and nicely elliptic.

By hypothesis (i) of Theorem 3.5, we know that the orbit $P_{3}$ spans an embedded disk whose interior is transverse to $R_{\lambda}$. Applying Theorem 3.10 to the orbit $P=P_{3}$ we obtain an embedded disk $\mathcal{D}$ spanning $P_{3}$ with special properties. The vector field $V$ that parametrizes the characteristic distribution $(\xi \cap T \mathcal{D})^{d \lambda}$ points outwards at $\partial \mathcal{D}$ and has precisely one zero $e$ in the interior of $\mathcal{D}$, which is a positive nicely elliptic singularity and a source for the dynamics of $V$.

\subsubsection{The Bishop family}

In this section, we follow the exposition of [HSa11] and [HLSa15].

Let $\mathcal{D}$ be the disk obtained by applying Theorem 3.10 to the orbit $P_{3}$. Identifying $S^{3}$ with $\{0\} \times S^{3} \subset \mathbb{R} \times S^{3}$ we identify $\mathcal{D}$ with $\{0\} \times \mathcal{D} \subset \mathbb{R} \times S^{3}$. Following ideas of [Hof93, HWZ95a, HWZ99a], we consider the boundary value problem

$$
\left\{\begin{array}{l}
\tilde{u}=(a, u): \mathbb{D} \rightarrow \mathbb{R} \times S^{3} \text { is an embedding, } \\
d \tilde{u} \cdot i=\tilde{J}(\tilde{u}) \cdot d \tilde{u} \\
a \equiv 0 \text { on } \partial \mathbb{D}, \quad u(\partial \mathbb{D}) \subset \mathcal{D} \backslash\{e\} \\
u(\partial \mathbb{D}) \text { winds once positively around } e .
\end{array}\right.
$$

Here $i$ denotes the standard complex structure on $\mathbb{C}$ and $J$ is any complex structure in $\mathcal{J}(\xi, d \lambda)$. The disk $\mathcal{D}$ is oriented so that $\left.\lambda\right|_{\partial \mathcal{D}}>0$ and the loop $u(\partial \mathbb{D})$ is oriented by orienting $\partial \mathbb{D}$ counterclockwise.

Define, for any $J \in \mathcal{J}(\xi, d \lambda)$,

$$
\mathcal{M}(J)=\left\{\tilde{u}=(a, u): \mathbb{D} \rightarrow \mathbb{R} \times S^{3} \text { solution of }(3.4) \mid u(\partial \mathbb{D}) \cap \partial \mathcal{D}=\emptyset\right\} .
$$

It is proved in Hof93 that there exists $J \in \mathcal{J}(\xi, d \lambda)$ and a continuous map $\Psi:[0, \epsilon) \times \mathbb{D} \rightarrow \mathbb{R} \times S^{3}$ such that $\Psi:(0, \epsilon) \times \mathbb{D} \rightarrow \mathbb{R} \times S^{3}$ is a smooth 
embedding, $\Psi(0, \mathbb{D})=\{e\}$ and

$$
\Psi(\tau, \cdot): \mathbb{D} \rightarrow \mathbb{R} \times S^{3} \text { satisfies } 3.4 \text {, for } \tau \in(0, \epsilon) .
$$

Note that if $\tilde{u}$ is a solution of (3.4), then also $\tilde{u} \circ \varphi$ is a solution for every biholomorphism $\varphi: \mathbb{D} \rightarrow \mathbb{D}$. The group $G$ of all such biholomorphisms has dimension 3. It is proved in [Hof93] that for any $J \in \mathcal{J}(\xi, d \lambda)$, the linearization of $\partial_{\tilde{J}}$ at any $\tilde{u} \in \mathcal{M}(J)$ is surjective and its Fredholm index is 4. An application of the implicit function theorem turns $\mathcal{M}(J)$ into a 4-dimensional smooth manifold. One can show that $(\tilde{u}, \varphi) \mapsto \tilde{u} \circ \varphi$ is a smooth, proper and free right action of $G$ on $\mathcal{M}(J)$. This implies $2^{2}$ that $\Pi: \mathcal{M}(J) \rightarrow \frac{\mathcal{M}(J)}{G}$ is a smooth principal $G$-bundle with one dimensional base space $\frac{\mathcal{M}(J)}{G}$, where $\Pi$ is the quotient projection.

It is also proved in [Hof93] that if $\tilde{u}_{n} \in \mathcal{M}(J)$ satisfies $\tilde{u}_{n} \rightarrow \tilde{u}$ in $C_{\text {loc }}^{\infty}\left(\mathbb{D}, \mathbb{R} \times S^{3}\right)$ and $\tilde{u}$ is non-constant, then $\tilde{u}$ solves 3.4 .

Next, we want to parametrize the one dimensional base space $\frac{\mathcal{M}(J)}{G}$. To do so, we need some additional facts.

Fix $\tilde{u}_{0} \in \mathcal{M}(J)$ and let $t_{0}=\Pi\left(\tilde{u}_{0}\right) \in \frac{\mathcal{M}(J)}{G}$. It is proved in [Hof93] that if $s$ is a section of $\mathcal{M}(J) \rightarrow \frac{\mathcal{M}(J)}{G}$ defined around $t_{0}$ satisfying $s\left(t_{0}\right)=\tilde{u}_{0}$, then there exists a neighborhood $U$ of $t_{0}$ in $\frac{\mathcal{M}(J)}{G}$ such that the map

$$
\begin{aligned}
\Phi: U \times \mathbb{D} & \rightarrow \mathbb{R} \times S^{3} \\
(t, z) & \mapsto s(t)(z)
\end{aligned}
$$

is a smooth embedding onto its image.

Each leaf $l$ of the characteristic foliation is a trajectory of the vector field $V$. Since there are no singularities other than $e, \partial \mathcal{D}$ is the image of a Reeb trajectory and $V$ points outwards at $\partial \mathcal{D}$, the $\alpha$-limit of $l$ is the source $e$ and $l$ hits $\partial \mathcal{D}$ transversally in forward and finite time. Moreover, $l$ has finite length since $e$ is nicelly elliptic.

As proved in [Hof93, Lemma 19], the strong maximum principle ${ }^{3}$ implies that if $\tilde{u} \in \mathcal{M}(J)$, then $u(\partial \mathbb{D})$ intersects the leaves transversally. Since $u(\partial \mathbb{D})$ winds once around $e$ in $\mathcal{D}$, it hits every leaf exactly once.

Following HWZ95a, choose a leaf $l_{1}$ of the characteristic foliation and denote its length by $\bar{\tau}$. We can define a smooth map $\tau: \frac{\mathcal{M}(J)}{G} \rightarrow(0, \bar{\tau})$ as follows.

Fix $\tilde{u}=(a, u) \in \mathcal{M}(J)$. For any $\varphi \in G, u \circ \varphi(\partial \mathbb{D})$ intersect $l_{1}$ at the

${ }^{2} \mathrm{By}$ the caracterization of smooth principal bundles.

${ }^{3}$ a.k.a Hopf Lemma. 
same point $p_{*}(\tilde{u})$. Set

$$
\tau(\Pi(\tilde{u}))=\text { length of the piece of } l_{1} \text { connecting } e \text { to } p_{*}(\tilde{u}) \text {. }
$$

It follows from the existence of local embeddings as in 3.5 that $\tau$ is a local diffeomorphism.

Intersections The following result is a consequence of Theorem 4.4 of [HWZ95a].

Theorem 3.11 ([HWZ95a $]$ ). Let $\mathcal{Y} \subset \frac{\mathcal{M}}{G}$ be a connected component containing disks arbitrarily close to the constant $(0, e)$. If $\tilde{u}: \mathbb{D} \rightarrow \mathbb{R} \times M$ is a smooth map and $\tilde{u}_{k} \in \Pi^{-1}(\mathcal{Y})$ is a sequence satisfying $\tilde{u}_{k} \rightarrow \tilde{u}$ in $C^{\infty}$, then $\tilde{u}(\mathbb{D}) \cap\left(\mathbb{R} \times x_{3}(\mathbb{R})\right)=\emptyset$.

The following proposition is a consequence of the arguments in the proof of [HWZ95a, Theorem 4.4] and will be useful later.

Proposition 3.12. Let $J \in \mathcal{J}(\xi, d \lambda)$. Consider a point $z \in \partial \mathbb{D}$, an open neighborhood $U$ of $z$ in $\mathbb{D}$ and a sequence $\tilde{u}_{k}=\left(a_{k}, u_{k}\right): U \rightarrow \mathbb{R} \times S^{3}$ of $\tilde{J}$-holomorphic maps satisfying $u_{k}(U \cap \partial \mathbb{D}) \subset \mathcal{D} \backslash \partial \mathcal{D}, \forall k$. Assume $\tilde{u}=$ $(a, u): U \rightarrow \mathbb{R} \times S^{3}$ is a $\tilde{J}$-holomorphic map such that $\tilde{u}_{k} \rightarrow \tilde{u}$ in $C_{\text {loc }}^{\infty}(U)$ and $\int_{U} u^{*} d \lambda>0$. If $u(z) \in x_{3}(\mathbb{R})$, then for $k$ large enough, there exists $\delta_{k} \in U$ satisfying $u_{k}\left(\delta_{k}\right) \in x_{3}(\mathbb{R})$.

A proof of Proposition 3.12 can be found in [HLSa15, Corollary 6.11].

Generic almost complex structures Let $\Gamma \subset \mathbb{D}$ be a finite non empty set and consider the mixed boundary value problem

$$
\left\{\begin{array}{l}
\tilde{u}=(a, u): \mathbb{D} \backslash \Gamma \rightarrow \mathbb{R} \times S^{3} \text { is an embedding } \\
d \tilde{u} \cdot i=\tilde{J}(\tilde{u}) \cdot d \tilde{u} \\
a \equiv 0 \text { on } \partial \mathcal{D}, \quad u(\partial \mathbb{D}) \subset \mathcal{D} \backslash\{e\} \\
u(\partial \mathbb{D}) \text { winds once positively around } e \\
\int_{\mathbb{D} \backslash \Gamma} u^{*} d \lambda>0, \quad E(\tilde{u})<\infty \\
\text { Every } z \in \Gamma \text { is a negative puncture }
\end{array}\right.
$$

Here we do not fix the complex structure on $\mathbb{D}$.

The following proposition is a consequence of results in [HWZ99b]. 
Proposition 3.13. There exists a residual set $\mathcal{J}_{\text {gen }}^{2} \subset \mathcal{J}(\xi, d \lambda)$ such that the following holds. Assume $J \in \mathcal{J}_{\text {gen }}^{2}$ and $\tilde{u}$ is a $\tilde{J}$-holomorphic solution of (3.6) asymptotic to a closed Reeb orbit $P_{z}$ at each puncture $z \in \Gamma$. If $\mu\left(P_{z}\right) \geq 2, \forall z \in \Gamma$, then there exists at most one puncture in $\Gamma$ and if there is a puncture $z_{0}$, then $\mu\left(P_{z_{0}}\right)=2$. define

Let $\mathcal{J}_{\text {gen }}^{1} \subset \mathcal{J}(\xi, d \lambda)$ be the residual set obtained by Theorem 2.20 , and

$$
\mathcal{J}_{\text {reg }}:=\mathcal{J}_{\text {reg }}^{1} \cap \mathcal{J}_{\text {reg }}^{2}
$$

Limiting behavior of the Bishop family There exists $J \in \mathcal{J}(\xi, d \lambda)$ and a disk $\tilde{v}_{*}=(b, v) \in \mathcal{M}(J)$ arbitrarily close to the constant $(0, e)$ satisfying

$$
\tilde{v}(\mathbb{D}) \cap\left(\mathbb{R} \times x_{3}(\mathbb{R})\right)=\emptyset .
$$

Every such $\tilde{v}_{*}$ is automatically Fredholm regular for a Fredholm theory of pseudoholomorphic disks with boundary in the embedded surface $\{0\} \times \mathcal{D} \backslash$ $\{e\}$, which is totally real in $(\mathbb{R} \times M, \tilde{J})$, as proved in Hof93. If $J^{\prime} \in \mathcal{J}_{\text {reg }}$ is a $C^{\infty}$ small perturbation of $J$, then we can find a disk $\tilde{v}_{*} \in \mathcal{M}\left(J^{\prime}\right)$ as a $C^{\infty}$ small perturbation of $\tilde{v}_{*}$. Reverting the notation back to $J$ and $\tilde{v}_{*}$, it follows that we could have assumed $J \in \mathcal{J}_{\text {reg }}$ from the beginning.

Let $\mathcal{Y}$ be the connected component of $\frac{\mathcal{M}(J)}{G}$ containing $\Pi\left(\tilde{v}_{*}\right)$. The set of solutions $\tilde{u}$ of $(3.4)$ in $\Pi^{-1}(\mathcal{Y})$ satisfying $\tilde{u}(\mathbb{D}) \cap\left(\mathbb{R} \times x_{3}(\mathbb{R})\right)=\emptyset$ is closed in $\Pi^{-1}(\mathcal{Y})$. This follows from Theorem 3.11. Since it is also open, non-empty and $\Pi^{-1}(\mathcal{Y})$ is connected, every $\tilde{u} \in \Pi^{-1}(\mathcal{Y})$ satisfies $\tilde{u}(\mathbb{D}) \cap\left(\mathbb{R} \times x_{3}(\mathbb{R})\right)=\emptyset$.

Define

$$
\tau^{*}:=\sup _{\tilde{u} \in \Pi^{-1}(\mathcal{Y})} \tau(\tilde{u}) .
$$

Fix two other leaves $l_{i}$ and $l_{-1}$ of the characteristic foliation of $\mathcal{D}$ distinct of $l_{1}$ in such a way that $\left\{l_{1}, l_{i}, l_{-1}\right\}$ is ordered according to the Reeb flow along the boundary.

Now we consider a sequence $\tau_{n} \rightarrow \tau^{*}$ and choose disks $\tilde{u}_{n}=\left(a_{n}, u_{n}\right) \in$ $\pi^{-1}(\mathcal{Y})$ satisfying $\tau_{n}=\tau\left(\tilde{u}_{n}\right)$ and the normalization condition $u_{n}(1) \in l_{1}, u_{n}(i) \in$ $l_{i}$ and $u_{n}\left(l_{-1}\right) \in l_{-1}$. Define

$$
\Gamma_{0}=\left\{z \in \mathbb{D} \mid \exists n_{j} \rightarrow \infty \text { and } z_{j} \rightarrow z \text { such that }\left|d \tilde{u}_{n_{j}}\left(z_{j}\right)\right| \rightarrow \infty\right\} .
$$

We need the following Theorem of [HWZ95a].

Theorem 3.14 ([HWZ95a $]$ ). $\exists 0<\rho<1$ such that for every sequence 
$\tilde{u}_{n} \in \mathcal{M}(J)$ satisfying $\tilde{u}_{n}(w) \in l_{w}$ for $w \in\{1, i,-1\}$, we have

$$
\sup _{n} \sup _{\rho<|z| \leq 1}\left|d \tilde{u}_{n}(z)\right|<\infty \text {. }
$$

By Theorem 3.14, $\Gamma_{0} \subset \mathbb{D} \backslash \partial \mathbb{D}$.

Note that

$$
0<C:=\sup _{\tilde{u} \in \mathcal{M}(J)} E(\tilde{u})<T_{3} .
$$

Indeed, let $\tilde{u} \in \mathcal{M}(J)$. Since $\tilde{u}$ is nonconstant, we have $E(\tilde{u})>0$. Denote by $\mathcal{D}_{u}$ the component of $\mathcal{D} \backslash u(\partial \mathbb{D})$ which contains the singular point $e$. Recall that $\mathcal{D}$ is transverse to the Reeb vector field and oriented in such a way that $\left.d \lambda\right|_{F}>0$. Using Stokes theorem and $a(\partial \mathbb{D})=0$, we can estimate for any $\phi \in C^{\infty}(\mathbb{R},[0,1])$

$$
\begin{aligned}
\int_{\mathbb{D}} \tilde{u}^{*} d \lambda_{\phi} & =\int_{\partial \mathbb{D}} \phi(0) u^{*} \lambda=\int_{\mathcal{D}_{u}} \phi(0) d \lambda \\
& \leq \int_{\mathcal{D}_{u}} d \lambda<\int_{\mathcal{D}} d \lambda=\int_{P_{3}} \lambda=T_{3} .
\end{aligned}
$$

Passing to a subsequence, still denoted by $\tilde{u}_{n}$, we may assume $\Gamma_{0}$ is finite. This follows from (3.7) and from the same arguments used in the proof of Proposition 2.26. Thus, by Theorem 2.22, there exists a smooth $\tilde{J}$-holomorphic map

$$
\tilde{u}_{0}=\left(a_{0}, u_{0}\right): \mathbb{D} \backslash \Gamma_{0} \rightarrow \mathbb{R} \times S^{3}
$$

and a subsequence, still denoted by $\tilde{u}_{n}$, such that

$$
\tilde{u}_{n} \rightarrow \tilde{u}_{0}, \quad \text { in } C_{l o c}^{\infty}\left(\mathbb{D} \backslash \Gamma_{0}\right) \text {. }
$$

Arguing as in section 2.3.3, we conclude that $\Gamma_{0}$ consists of negative punctures of $\tilde{u}_{0}$.

Lemma 3.15. $\Gamma_{0} \neq \emptyset$.

Proof. Suppose $\Gamma_{0}=\emptyset$. Then $\tilde{u}_{n} \rightarrow \tilde{u}_{0}$ in $C_{l o c}^{\infty}\left(\mathbb{D}, \mathbb{R} \times S^{3}\right)$ and $\tilde{u}_{0}$ is non constant. As remarked before, this implies that $\tilde{u}_{0}$ solves (3.4). By Theorem 3.11. $u_{0}(\partial \mathbb{D}) \cap x_{3}(\mathbb{R})=\emptyset$. Thus, $\tilde{u}_{0} \in \Pi^{-1}(\mathcal{Y})$ and $\tau\left(\tilde{u}_{0}\right)=\tau^{*}$. We take a local section $s_{0}$ of $\mathcal{M}$ defined on a neighborhood $U_{0}$ of $\Pi\left(\tilde{u}_{0}\right)$ in $\frac{\mathcal{M}}{G}$ and define $\Phi_{0}: U_{0} \times \mathbb{D} \rightarrow \mathbb{R} \times S^{3}$ by $\Phi_{0}(t, z)=s_{0}(t)(z)$. As explained before, $\Phi_{0}$ is a smooth embedding into $\mathbb{R} \times S^{3}$. Thus, we can find elements $\tilde{u} \in \Pi^{-1}(\mathcal{Y})$ satisfying $\tau(\tilde{u})>\tau\left(\tilde{u}_{0}\right)=\tau^{*}$, a contradiction. 
Lemma 3.16. $\tilde{u}_{0}$ is an embedding, $a_{0} \equiv 0$ on $\partial \mathbb{D}$ and $u_{0}(\partial \mathbb{D}) \subset \mathcal{D} \backslash\{e\}$ winds once and positively around $e$.

Proof. Since $\tilde{u}_{n} \rightarrow \tilde{u}_{0}$ in $C_{\text {loc }}^{\infty}, a_{n} \equiv 0$ on $\partial \mathbb{D}$ and $u_{n}(\partial \mathbb{D}) \subset \mathcal{D} \backslash\{e\}$ winds once and positively around $e$ for every $n$, we have $a_{0} \equiv 0$ on $\partial \mathbb{D}$ and $u_{0}(\partial \mathbb{D}) \subset$ $\mathcal{D} \backslash\{e\}$ winds once and positively around $e$. Using the fact that $a_{0}$ satisfies the strong maximum principle, one can show that $\tilde{u}_{0}$ is an embedding near $\partial \mathbb{D}$. It follows from results in [McD91] that self-intersections or critical points of $\tilde{u}_{0}$ would imply self-intersections or critical points of $\tilde{u}_{n}$ for $n$ large, which contradicts the fact that $\tilde{u}_{n}$ is an embedding for every $n$. Thus, $\tilde{u}_{0}$ is an injective immersion. It follows from Lemma 2.14 that $\tilde{u}_{0}$ is an embedding.

Fix any $z_{0} \in \Gamma_{0}$ and let $P_{0}=\left(x_{0}, T_{0}\right)$ be the asymptotic limit of $\tilde{u}_{0}$ at $z_{0}$. As in Section 2.3.4, we define the mass of $z_{0}$ by

$$
m\left(z_{0}\right)=\lim _{\epsilon \rightarrow 0^{+}} m_{\epsilon}\left(z_{0}\right),
$$

where

$$
m_{\epsilon}\left(z_{0}\right)=\lim _{n \rightarrow \infty} \int_{B_{\epsilon}\left(z_{0}\right)} u_{n}^{*} d \lambda .
$$

Now we proceed as in the soft rescaling done in Section 2.3.4. Fix $\epsilon>0$ such that

$$
m_{\epsilon}\left(z_{0}\right)-m\left(z_{0}\right) \leq \frac{\sigma(C)}{2} .
$$

Choose $z_{n}$ defined by $a_{n}\left(z_{n}\right)=\inf \left(a_{n}\left(B_{\epsilon}\left(z_{0}\right)\right)\right)$ and let $0<\delta_{n}<\epsilon$ be defined by

$$
\int_{B_{\epsilon}\left(z_{0}\right) \backslash B_{\delta_{n}}\left(z_{n}\right)} u_{n}^{*} d \lambda=\sigma(C) .
$$

It follows that $z_{n} \rightarrow z_{0}$ and, up to a subsequence, $\delta_{n} \rightarrow 0$. Take $R_{n} \rightarrow+\infty$ such that $\delta_{n} R_{n}<\frac{\epsilon}{2}$ and define

$$
\begin{aligned}
\tilde{v}_{n}=\left(b_{n}, v_{n}\right): B_{R_{n}}(0) & \rightarrow \mathbb{R} \times S^{3} \\
z & \mapsto\left(a_{n}\left(z_{n}+\delta_{n} z\right)-a_{n}\left(z_{n}+2 \delta_{n}\right), u_{n}\left(z_{n}+\delta_{n} z\right)\right)
\end{aligned}
$$

The sequence $\tilde{v}_{n}$ is a germinating sequence, according to Definition 2.25 . Let

$$
\Gamma_{1}=\left\{z \in \mathbb{C} \mid \exists z_{j} \rightarrow z \text { and subsequence } \tilde{u}_{n_{j}} \text { s.t. }\left|d \tilde{u}_{n_{j}}\left(z_{j}\right)\right| \rightarrow \infty\right\} .
$$

Passing to a subsequence, we can assume $\Gamma_{1}$ is finite. Let

$$
\tilde{v}=(b, v): \mathbb{C} \backslash \Gamma_{1} \rightarrow \mathbb{R} \times S^{3}
$$


be a limit of $\tilde{v}_{n}$, according to Definition 2.27. Then $\tilde{v}$ has a unique positive puncture at $\infty$ and $\tilde{v}$ is asymptotic to $P_{0}$ at $\infty$. Thus, using Lemma 2.37, we obtain the following.

Lemma 3.17. If $z \in \Gamma_{0}$ and $\tilde{u}_{0}$ is asymptotic to $P$ at $z$, then $\mu(P) \geq 2$.

Now we divide our analysis into two cases:

1) $\pi \cdot d u_{0} \equiv 0$

2) $\int_{\mathbb{D} \backslash \Gamma_{0}} u_{0}^{*} d \lambda>0$

\subsubsection{The Case $\pi \cdot d u_{0} \equiv 0$}

In this section, we use the same notation as in 3.3 .2 .

Proposition 3.18. Assume $\pi \cdot d u_{0} \equiv 0$. After reparametrizing, we can asssume $\Gamma_{0}=\{0\}$ and

$$
\tilde{u}_{n} \rightarrow F_{P_{3}}, \quad \text { in } C_{l o c}^{\infty}\left(\mathbb{D} \backslash\{0\}, \mathbb{R} \times S^{3}\right),
$$

where $F_{P_{3}}$ denotes the map $e^{2 \pi(s+i t)} \mapsto\left(T_{3} s, x_{3}\left(T_{3} t\right)\right)$ on $\mathbb{D} \backslash\{0\}$.

Proof. Since $\pi \cdot d u_{0} \equiv 0$, there exists a Reeb trajectory $\tilde{x}$ such that $u_{0}(\mathbb{D} \backslash$ $\left.\Gamma_{0}\right) \subset \tilde{x}(\mathbb{R})$. It follows from Lemma 3.16 that $\tilde{x}$ is periodic and $\tilde{x}(\mathbb{R})=$ $u_{0}(\partial \mathbb{D}) \subset \mathcal{D}$. By the properties of the disk $\mathcal{D}$ given by Proposition 3.10 , we have $\tilde{x}(\mathbb{R})=x_{3}(\mathbb{R})$. Consider the map $F: \mathbb{D} \backslash\{0\} \rightarrow \mathbb{R} \times S^{3}$ given by

$$
z=e^{2 \pi(s+i t)} \mapsto\left(T_{3} s, x_{3}\left(T_{3} t\right)\right)
$$

One can prove using Carleman's Similarity Principle that $\exists k \in \mathbb{Z}^{+}$and a holomorphic map $\varphi: \mathbb{D} \rightarrow \mathbb{D}$ satisfying $\varphi(\partial \mathbb{D})=\partial \mathbb{D}, \Gamma_{0}=\varphi^{-1}(0)$ and $\left.\varphi\right|_{\partial \mathbb{D}}: \partial \mathbb{D} \rightarrow \partial \mathbb{D}$ has degree $k$, and such that

$$
\tilde{u}_{0}=F \circ \varphi
$$

Since $T_{3}$ is the minimal positive period of $x_{3}, u_{0}(\partial \mathbb{D})$ winds $k$ times around $e$ in $\mathcal{D}$. It follows from Lemma 3.16 that $k=1$ and $\varphi$ is a biholomorphism.

Let $\tilde{v}_{n}=\left(b_{n}, v_{n}\right)$ be the germinating sequence defined in $(3.8)$ for $z_{0}=0$ and let $\Gamma_{1}$ be defined as in 3.9 . Let $\tilde{v}:=(b, v): \mathbb{C} \backslash \Gamma_{1} \rightarrow \mathbb{R} \times S^{3}$ be a limit of the sequence $\tilde{v}_{n}$. Then $\tilde{v}$ has a unique positive puncture at $\infty$ and the points in $\Gamma_{1}$ are negative punctures. By Proposition 3.18, we know that $\tilde{v}$ is asymptotic to $P_{3}$ at $\infty$. Also, if $\Gamma_{1} \neq \emptyset$, then $0 \in \Gamma_{1}$. This is because $b_{n}(0)=\inf b_{n}\left(B_{R_{n}}(0)\right)$ and the points in $\Gamma_{1}$ are negative punctures. 

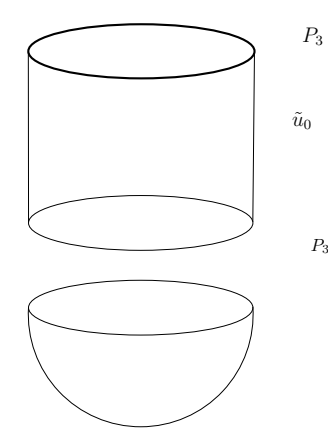

Figure 3.2: Case $\pi \cdot d u_{0} \equiv 0$ and $\Gamma_{1}=\emptyset$

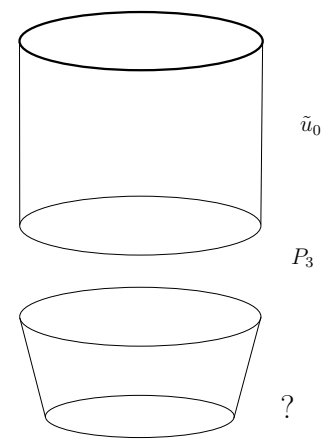

Figure 3.3: Case $\pi \cdot d u_{0} \equiv 0$ and $\Gamma_{1} \neq \emptyset$

Lemma 3.19. $\int_{\mathbb{C} \backslash \Gamma_{1}} v^{*} d \lambda>0$.

Proof. Suppose that $\pi \circ d v$ vanishes identically. Then, by Theorem 2.6, there exists a polynomial $p: \mathbb{C} \rightarrow \mathbb{C}$ such that $\Gamma_{1}=p^{-1}(0)$ and $\tilde{v}=F_{P_{3}} \circ p$. Since $P_{3}$ is prime, $p$ must have degree 1 . This implies $\Gamma_{1}=\{0\}$, a contradiction with Proposition 2.30 .

Corollary 2.15 together with the fact that $P_{3}$ is simply covered implies that $\tilde{v}=(b, v)$ is somewhere injective.

Thus, we can apply Theorem 2.20 to conclude

$$
1 \leq \mu\left(P_{3}\right)-\sum_{z \in \Gamma_{1}} \mu\left(P_{z}\right)-\chi_{S^{2}}+\# \Gamma_{1}+1 \leq 2-\sum_{z \in \Gamma_{1}} \mu\left(P_{z}\right)+\# \Gamma_{1},
$$

where $P_{z}$ is the asymptotic limit of $\tilde{v}$ at $z$. The only possibilities for the set of punctures $\Gamma_{1}$ are

- Either $\Gamma_{1}=\emptyset \quad$ or

- $\Gamma_{1}=\{0\}$ and $\mu\left(P_{0}\right)=2$.

The bubbling-off tree in the case $\Gamma_{1}=\{0\}$ Assume that $\Gamma_{1}=\{0\}$ and $\mu\left(P_{0}\right)=2$. Let $\tilde{w}: \mathbb{C} \backslash \Gamma_{2} \rightarrow \mathbb{R} \times S^{3}$ be the unique vertex immediately below $\tilde{v}$ in the bubbling-off tree $\mathcal{B}$ associated with the germinating sequence $\tilde{v}_{n}$ and the limit $\tilde{v}$.

We can apply Lemma 2.39 to the curve $\tilde{w}$ to conclude that every asymptotic limit of $\tilde{w}$ has Conley-Zehnder index equal to 2 . Using this fact we can prove the following lemma.

Lemma 3.20. $\tilde{w}: \mathbb{C} \backslash \Gamma_{2} \rightarrow \mathbb{R} \times S^{3}$ is somewhere injective. 
Proof. Arguing by contradiction, we assume that $\tilde{w}$ is not somewhere injective. Then there exist a polynomial $p: \mathbb{C} \rightarrow \mathbb{C}$ and a somewhere injective finite energy sphere $\tilde{u}: \mathbb{C} \backslash \Gamma^{\prime} \rightarrow \mathbb{R} \times S^{3}$ such that $\operatorname{deg} p \geq 1, p^{-1}\left(\Gamma^{\prime}\right)=\Gamma_{2}$ and $\tilde{w}$ factors as

$$
\tilde{w}=\tilde{u} \circ p .
$$

Let $P$ be the asymptotic limit of $\tilde{w}$ at $\infty$ and $P_{\infty}$ be the asymptotic limit of $\tilde{u}$ at $\infty$. Then $P_{\infty}^{\operatorname{deg} p}=P$. Using Lemma 1.9 (iii) and $\mu(P)=2$, we conclude that $\operatorname{deg} p=2$ and $\mu\left(P_{\infty}\right)=1$. By Theorem 2.20 applied to $\tilde{u}$, we have the estimate

$$
0 \leq \mu\left(P_{\infty}\right)-\sum_{z^{\prime} \in \Gamma^{\prime}} \mu\left(P_{z^{\prime}}\right)-\chi_{S^{2}}+\# \Gamma^{\prime}+1=1-\sum_{z^{\prime} \in \Gamma^{\prime}} \mu\left(P_{z^{\prime}}\right)-2+\# \Gamma^{\prime}+1
$$

where $P_{z^{\prime}}$ is the asymptotic limit of $\tilde{u}$ at $z^{\prime}$. We conclude $\sum_{z^{\prime} \in \Gamma^{\prime}} \mu\left(P_{z^{\prime}}\right) \leq \# \Gamma^{\prime}$ and consequently $\mu\left(P_{z^{\prime}}\right) \leq 1, \forall z^{\prime} \in \Gamma^{\prime}$.

Suppose that $\zeta \in \Gamma$ is a regular point of $p$. Then $p$ is a biholomorphism in a neighborhood of $\zeta$ and, if $z^{\prime}=p(\zeta)$, then

$$
P_{\zeta}=P_{z^{\prime}}
$$

This leads to the contradiction $\mu\left(P_{\zeta}\right)=2>1 \geq \mu\left(P_{z^{\prime}}\right)$. We conclude that if $\Gamma_{2} \neq \emptyset$, it consists of critical points of $p$. Since $\operatorname{deg} p=2$, we have $\#$ Crit $p=1$. It follows that $\# \Gamma_{2} \leq 1$ and consequently $\# \Gamma^{\prime} \leq 1$.

By Proposition 2.30, we know that $\int_{\mathbb{C} \backslash \Gamma} w^{*} d \lambda>0$. Consequently, we have $\int_{\mathbb{C} \backslash \Gamma^{\prime}} u^{*} d \lambda>0$. By Theorem 2.20 applied to $\tilde{u}$, we have the estimate

$$
1 \leq \mu\left(P_{\infty}\right)-\sum_{z^{\prime} \in \Gamma^{\prime}} \mu\left(P_{z^{\prime}}\right)-2+\# \Gamma^{\prime}+1=-\sum_{z^{\prime} \in \Gamma^{\prime}} \mu\left(P_{z^{\prime}}\right)+\# \Gamma^{\prime} .
$$

We conclude that $\Gamma^{\prime}=\left\{z^{\prime}\right\}$ and $\mu\left(P_{z^{\prime}}\right)=0$. It follows that $\Gamma_{2}=\{\zeta\}$, $p(\zeta)=z^{\prime}, p^{\prime}(\zeta)=0$ and

$$
P_{z^{\prime}}^{2}=P_{\zeta}
$$

By lemma 1.9 this leads to a contradiction. We have proved that $\tilde{w}$ is somewhere injective.

By Theorem 2.20, we have the estimate

$$
0 \leq \operatorname{ind}(\tilde{w}) \leq 2-2 \# \Gamma_{2}-2+\# \Gamma_{2}+1=1-\# \Gamma_{2} .
$$

Thus, we conclude that $\# \Gamma_{2} \leq 1$ and consequently, by Lemma 2.30 , we have 


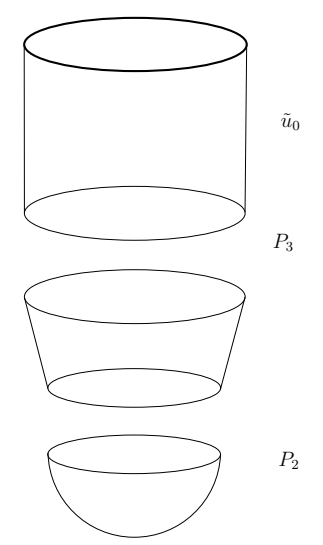

Figure 3.4: Case $\pi \cdot d u_{0} \equiv 0$ and $\Gamma_{1} \neq \emptyset$

$\int_{\mathbb{C} \backslash \Gamma_{2}} w^{*} d \lambda>0$. Again by Theorem 2.20 we obtain

$$
1 \leq \operatorname{ind}(\tilde{w}) \leq 1-\# \Gamma_{2}
$$

Thus, $\# \Gamma_{2}=0$ and $\tilde{w}$ is the only leaf in the bubbling-off tree. By Proposition 2.38 , the leaf is asymptotic to an orbit $\bar{P}=(\bar{x}, \bar{T})$ such that $\mu(\bar{P})=2$ and $P_{3}$ do not intersect the image of $w$. Then $\bar{P}$ is not linked to $P_{3}$. Since $\bar{T}<T_{3}$, it follows from the hypotheses of Theorem 3.5 that

$$
\bar{P}=P_{2} \text {. }
$$

So far, we have a $\tilde{J}$-holomorphic cylinder $\tilde{v}: \mathbb{C} \backslash\{0\} \rightarrow \mathbb{R} \times S^{3}$ asymptotic to $P_{3}$ at its positive puncture $\infty$ and to $P_{2}$ at its negative puncture 0 , and a $\tilde{J}$-holomorphic plane asymptotic to $P_{2}$.

The bubbling off tree obtained in the case $\pi \cdot d u_{0} \equiv 0 \quad$ In conclusion, in the case $\pi \cdot d u_{0} \equiv 0$, we have two possibilities:

1. Either $\Gamma_{1}=\emptyset$ and $\tilde{v}: \mathbb{C} \rightarrow \mathbb{R} \times S^{3}$, or

2. $\Gamma_{1}=\{0\}, \tilde{v}: \mathbb{C} \backslash\{0\} \rightarrow \mathbb{R} \times S^{3}$ is asymptotic to $P_{3}$ at its positive puncture $\infty$ and to $P_{2}$ at its negative puncture $z=0$. In this case we also obtain a $\tilde{J}$-holomorphic plane $\tilde{w}: \mathbb{C} \rightarrow \mathbb{R} \times S^{3}$ asymptotic to $P_{2}$.

We continue the analysis of the case $\pi \circ d u_{0} \equiv 0$ in Chapter 4. In the case $\Gamma_{1}=\emptyset$, we show that $\tilde{v}$ belongs to a 1 -parameter family of $J$-holomorphic planes asymptotic to $P_{3}$, whose projection foliates a region in $S^{3}$. This is the first step to obtain the $3-2-1$ foliation. In the case $\Gamma_{1}=\{0\}$, we apply the Gluing theorem 5.2 to the cylinder $\tilde{v}: \mathbb{C} \backslash\{0\} \rightarrow \mathbb{R} \times S^{3}$ and the plane 
$\tilde{w}: \mathbb{C} \rightarrow \mathbb{R} \times S^{3}$ to obtain a 1 -parameter family of $\tilde{J}$-holomorphic planes asymptotic to $P_{3}$.

\subsubsection{The case $\int_{\mathbb{D} \backslash \Gamma_{0}} u_{0}^{*} d \lambda>0$}

We continue using the notation of 3.3 .2 . In this case, $\left(\tilde{u}_{0}, \tilde{J}\right)$ is a solution of (3.6). Remember we have assumed $J \in \mathcal{J}_{\text {reg }}^{2}$, where $\mathcal{J}_{\text {reg }}^{2}$ is given by Proposition 3.13. By Lemma 3.15, we know that $\Gamma_{0} \neq \emptyset$ and, if $z \in \Gamma_{0}$ and $\tilde{u}_{0}$ is asymptotic to $P$ at $z$, then $\mu(P) \geq 2$. Thus, Proposition 3.13 implies that $\Gamma_{0}$ consists of a single point $z=0$ and $\mu\left(P_{0}\right)=2$, where $P_{0}$ is the asymptotic limit of $\tilde{u}_{0}$ at 0 .

Proposition 3.21. $u_{0}(\partial \mathbb{D}) \cap x_{3}(\mathbb{R})=\emptyset$.

Proof. Assume there exists $z \in \partial \mathbb{D}$ such that $u_{0}(z) \in x_{3}(\mathbb{R})$. By Lemma 3.12 applied to the sequence $\tilde{u}_{n}$, we obtain a sequence $\left\{\delta_{k}\right\}$ in $\mathbb{D} \backslash\{0\}$ satisfying $u_{n}\left(\delta_{n}\right) \in x_{3}(\mathbb{R})$, for $n$ large enough. It is a contradiction, since $u_{n}(\mathbb{D}) \cap$ $x_{3}(\mathbb{R})=\emptyset, \forall n$.

Consider the germinating sequence $\tilde{v}_{n}=\left(b_{n}, v_{n}\right): \mathbb{C} \backslash \Gamma_{1} \rightarrow \mathbb{R} \times S^{3}$ as defined in $(3.8)$, and let $\tilde{v}=(b, v)$ be a limit of the sequence $\tilde{v}_{n}$. Then $\tilde{v}$ satisfies the hypotheses of Lemma 2.39 and it follows that for all $z \in \Gamma_{1} \cup\{\infty\}$,

$$
\mu\left(P_{z}\right)=2,
$$

where $P_{z}$ is the asymptotic limit of $\tilde{v}$ at $z$. Using Lemma 3.20 , we conclude that $\tilde{v}$ is somewhere injective.

By Theorem 2.20, we have the estimate

$$
\begin{aligned}
0 \leq \operatorname{ind}(\tilde{v}) & \leq \mu\left(P_{2}\right)-\mu^{-}(\tilde{v})-\chi_{S^{2}}+\# \Gamma_{1}+1 \\
& =2-2 \# \Gamma_{1}-2+\# \Gamma_{1}+1 \\
& =1-2 \# \Gamma_{1} .
\end{aligned}
$$

We conclude that $\# \Gamma_{1} \leq 1$ and consequently, by Proposition 2.30, $\int_{\mathbb{C} \backslash \Gamma_{1}} v^{*} d \lambda>$ 0 . By Theorem 2.20 again, we have the estimate

$$
1 \leq \operatorname{ind}(\tilde{v}) \leq 1-2 \# \Gamma_{1} .
$$

Hence, $\# \Gamma_{1}=0$.

Let $P_{0}=\left(x_{0}, T_{0}\right)$ be the asymptotic limit of $\tilde{v}$ at $\infty$. It follows from Proposition 2.38 that $P_{0}$ is not linked to $P_{3}$. Also, $T_{0}<T_{3}$ and $\mu\left(P_{0}\right)=2$. By the hypotheses of Theorem 3.5, $P_{2}$ is the only orbit satisfying these properties. 


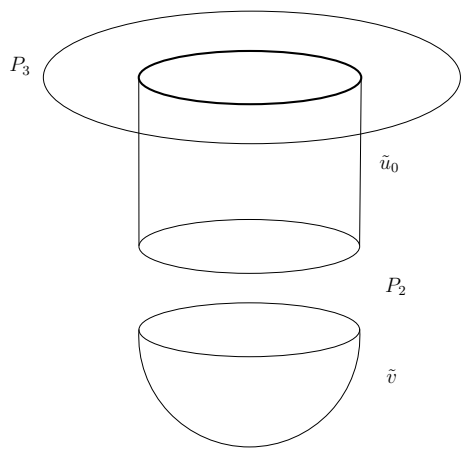

Figure 3.5: Case $\int_{\mathbb{D} \backslash \Gamma_{0}} u_{0}^{*} d \lambda>0$

Thus,

$$
P_{0}=P_{2} \text {. }
$$

We continue the analysis of the case $\int_{\mathbb{D} \backslash \Gamma_{0}} u_{0}^{*} d \lambda>0$ in Section 5.2 . 
74 CHAPTER 3. 3-2-1 FOLIATIONS AND THE MAIN THEOREM 


\section{Chapter 4}

\section{Proof of Theorem 3.5 in the case $\pi \cdot d u_{0} \equiv 0$}

Following Section 3.3.2, we consider a sequence $\tilde{u}_{n}=\left(a_{n}, u_{n}\right) \in \mathcal{M}(J)$ and a $\tilde{J}$-holomorphic map

$$
\tilde{u}_{0}=\left(a_{0}, u_{0}\right): \mathbb{D} \backslash \Gamma_{0} \rightarrow \mathbb{R} \times S^{3}
$$

such that, passing to a subsequence, $\tilde{u}_{n} \rightarrow \tilde{u}_{0}$ in $C_{l o c}^{\infty}\left(\mathbb{D} \backslash \Gamma_{0}\right)$. In this chapter we assume

$$
\pi \circ d u_{0} \equiv 0
$$

With this assumption, by Proposition 3.18 , we have

$$
\tilde{u}_{0}=F_{P_{3}} \text {, }
$$

where $F_{P_{3}}$ denotes the cylinder $e^{2 \pi(s+i t)} \mapsto\left(T_{3} s, x_{3}\left(T_{3} t\right)\right)$ in $\mathbb{D} \backslash\{0\}$.

Fix $\epsilon>0$ such that $m_{\epsilon}(0)-m(0) \leq \frac{\sigma(C)}{2}$ and choose $z_{n}$ defined by $a_{n}\left(z_{n}\right)=\inf \left(a_{n}\left(B_{\epsilon}(0)\right)\right)$. We also choose $0<\delta_{n}^{2}<\epsilon$ satisfying

$$
\int_{B_{\epsilon}(0) \backslash B_{\delta_{n}}\left(z_{n}\right)} u_{n}^{*} d \lambda=\sigma(C) .
$$

It follows that $z_{n} \rightarrow 0$ and, up to a subsequence, $\delta_{n} \rightarrow 0$. Take $R_{n} \rightarrow \infty$ such that $\delta_{n} R_{n}<\frac{\epsilon}{2}$ and define

$$
\begin{aligned}
\tilde{v}_{n}=\left(b_{n}, v_{n}\right): B_{R_{n}}(0) & \rightarrow \mathbb{R} \times S^{3} \\
z & \mapsto\left(a_{n}\left(z_{n}+\delta_{n} z\right)-a_{n}\left(z_{n}+2 \delta_{n}\right), u_{n}\left(z_{n}+\delta_{n} z\right)\right)
\end{aligned}
$$


The sequence $\tilde{v}_{n}$ is a germinating sequence as in Definition 2.25. Let

$$
\Gamma_{1}=\left\{z \in \mathbb{C} \mid \exists z_{j} \rightarrow z \text { and subsequence } \tilde{u}_{n_{j}} \text { s.t. }\left|d \tilde{u}_{n_{j}}\left(z_{j}\right)\right| \rightarrow \infty\right\} .
$$

Passing to a subsequence, we can assume $\# \Gamma_{1}<\infty$. Let

$$
\tilde{v}=(b, v): \mathbb{C} \backslash \Gamma_{1} \rightarrow \mathbb{R} \times S^{3}
$$

be a limit of the germinating sequence $\tilde{v}_{n}$ as in Definition 2.27. Then $\tilde{v}$ has a unique positive puncture at $\infty$ and the points of $\Gamma_{1}$ are negative punctures. Also, $\tilde{v}$ is asymptotic to $P_{3}$ at $\infty$. As proved in Section 3.3.3, we have two possibilities for $\Gamma_{1}$ :

- Either $\Gamma_{1}=\emptyset$ and $\tilde{v}$ is a plane asymptotic to $P_{3}$

- or $\Gamma_{1}=\{0\}$ and $\tilde{v}$ is a cylinder asymptotic to $P_{3}$ at its positive puncture and to $P_{2}$ at its negative puncture.

\subsection{Foliating a solid torus in the case $\Gamma_{1}=\emptyset$}

In this case $\tilde{v}: \mathbb{C} \rightarrow \mathbb{R} \times S^{3}$ is a $\tilde{J}$-holomorphic finite energy plane asymptotic to the orbit $P_{3}$. It follows from Lemma 2.34 that

$$
\operatorname{wind}_{\pi}(\tilde{v})=0
$$

As a consequence of the definition of wind $_{\pi}$, we conclude

$$
\mathbb{R} X_{v(z)} \oplus d v(z)\left(T_{z} \mathbb{C}\right)=T_{v(z)} S^{3}, \forall z \in \mathbb{C},
$$

proving that $v$ is an immersion transverse to the Reeb flow. In particular, this implies that $\tilde{v}$ is an immersion. Now we prove that $\tilde{v}$ is an embedding. Since $\tilde{v}$ is an immersion, it is enough to prove that $\tilde{v}$ is injective to conclude that it is an embedding in any closed ball $\bar{B}_{R^{\prime}} \subset \mathbb{C}, R^{\prime}>0$. Let $\Delta \subset \mathbb{C} \times \mathbb{C}$ be the diagonal and consider the set

$$
D:=\left\{\left(z_{1}, z_{2}\right) \in \mathbb{C} \times \mathbb{C} \backslash \Delta \mid \tilde{v}\left(z_{1}\right)=\tilde{v}\left(z_{2}\right)\right\}
$$

$D$ consists only of isolated points in $\mathbb{C} \times \mathbb{C} \backslash \Delta$. Indeed, if $D$ has a limit point in $\mathbb{C} \times \mathbb{C} \backslash \Delta$, then we find, using Carleman's similarity principle as in [HWZ95b], a polynomial $p$ of degree at least 2 and a $\tilde{J}$-holomorphic map $f: \mathbb{C} \rightarrow \mathbb{R} \times S^{3}$ such that $\tilde{v}=f \circ p$. This forces zeros of $d \tilde{v}$, contradicting the fact that $\tilde{v}$ is an immersion. If $D \neq \emptyset$, using positivity and stability of intersections of pseudo-holomorphic immersions, we obtain self-intersections 


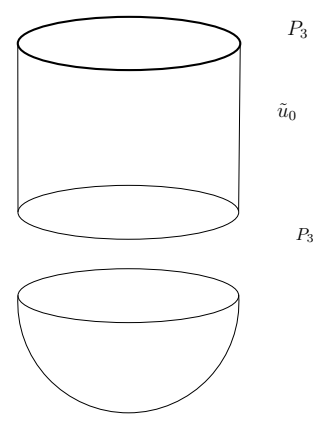

Figure 4.1: Case $\pi \cdot d u_{0} \equiv 0$ and $\Gamma_{1}=\emptyset$

of the maps $\tilde{v}_{n}$. However, we know that each $\tilde{u}_{n}$, and consequently each $\tilde{v}_{n}$ is an embedding. It follows, form Theorem 2.11, Lemma 2.14 and the fact that the orbit $P_{3}$ is prime, that $\tilde{v}$ is an embedding near the boundary, that is, there exists $R>0$ such that $\tilde{v}^{-1}\left(\tilde{v}\left(B_{R}\right)\right)=B_{R}$ and $\left.\tilde{v}\right|_{\mathbb{C} \backslash B_{R}}$ is an embedding. This shows that $\tilde{v}$ is an embedding.

A family of planes asymptotic to $\boldsymbol{P}_{\mathbf{3}}$ The following Theorem is proved in HWZ99b.

Theorem 4.1 ([HWZ99b]). Assume that the $\tilde{J}$-holomorphic finite energy plane $\tilde{w}=(d, w): \mathbb{C} \rightarrow \mathbb{R} \times S^{3}$ is an embedding asymptotic to a nondegenerate simply covered orbit $P=(x, T)$ satisfying $\mu(P)=3$. Then there exists $\epsilon>0$ and an embedding

$$
\tilde{\Phi}=(a, \Phi):(-\epsilon, \epsilon) \times \mathbb{C} \rightarrow \mathbb{R} \times S^{3}
$$

so that

(i) $\tilde{\Phi}(0, \cdot)=\tilde{w}$;

(ii) For any $\tau \in(-\epsilon, \epsilon)$, the map $\tilde{\Phi}_{\tau}:=\tilde{\Phi}(\tau, \cdot): \mathbb{C} \rightarrow \mathbb{R} \times S^{3}$ is an embedded finite energy $\tilde{J}$-holomorphic plane asymptotic to $P$;

(iii) $\Phi(\tau, \mathbb{C}) \cap P=\emptyset, \forall \tau$ and the map $\Phi:(-\epsilon, \epsilon) \times \mathbb{C} \rightarrow S^{3} \backslash P$ is an embedding;

(iv) Let $\tilde{w}_{n}$ be a sequence of embedded fast finite energy $\tilde{J}$-holomorphic planes all asymptotic to $P$ satisfying $\tilde{w}_{n} \rightarrow \tilde{w}$ in $C_{\text {loc }}^{\infty}(\mathbb{C})$ as $n \rightarrow+\infty$. Then there exist sequences $A_{n}, B_{n}$ in $\mathbb{C}$ with $A_{n} \rightarrow 1, B_{n} \rightarrow 0, c_{n}$ in $\mathbb{R}$ with $c_{n} \rightarrow 0$ and $\tau_{n}$ in $(-\epsilon, \epsilon)$ with $\tau_{n} \rightarrow 0$ such that

$$
\tilde{w}_{n}(z)=\left(a_{\tau_{n}}\left(A_{n} z+B_{n}\right)+c_{n}, \Phi_{\tau_{n}}\left(A_{n} z+B_{n}\right)\right)
$$


for sufficiently large $n$.

By applying the above theorem to the plane $\tilde{v}$ we obtain a maximal 1parameter family of finite energy planes asymptotic to the orbit $P_{3}$

$$
\tilde{v}_{\tau}=\left(b_{\tau}, v_{\tau}\right), \quad \tau \in\left(\tau_{-}, \tau_{+}\right) .
$$

The family satisfies $v_{\tau_{1}}(\mathbb{C}) \cap v_{\tau_{2}}(\mathbb{C})=\emptyset, \forall \tau_{1} \neq \tau_{2}$. Indeed, if there are $\tau_{1} \neq \tau_{2}$ such that $v_{\tau_{1}}(\mathbb{C}) \cap v_{\tau_{2}}(\mathbb{C}) \neq \emptyset$, then $v_{\tau_{1}}(\mathbb{C})=v_{\tau_{2}}(\mathbb{C})$ and we would obtain an $S^{1}$-family of embedded planes that provides an open book decomposition 1 with disk-like pages to $S^{3}$, with binding orbit $P_{3}$. Since each plane in the family is fast and consequently transverse to the Reeb flow, it clearly contradicts the fact that $P_{2}$ and $P_{1}$ are not linked to $P_{3}$.

Now we describe how the family $\left\{v_{\tau}\right\}$ breaks as $\tau \rightarrow \tau_{ \pm}$. We assume that $\tau_{-}=0$ and $\tau_{+}=1$ and $\tau$ strictly increases in the direction of $R_{\lambda}$.

\section{Limiting behavior}

Proposition 4.2. Consider a sequence $\tilde{v}_{n}:=\tilde{v}_{\tau_{n}}$ in the family $\left\{\tilde{v}_{\tau}\right\}$ satisfying $\tau_{n} \rightarrow 0^{+}$. Then there exists a $\tilde{J}$-holomorphic finite energy cylinder $\tilde{u}_{r}$ : $\mathbb{C} \backslash\{0\} \rightarrow \mathbb{R} \times S^{3}$ which is asymptotic to $P_{3}$ at the positive puncture $\infty$ and to $P_{2}$ at its negative puncture and a finite energy $\tilde{J}$-holomorphic plane $\tilde{u}_{q}: \mathbb{C} \rightarrow$ $\mathbb{R} \times S^{3}$ asymptotic to $P_{2}$ at $\infty$, such that, after suitable reparametrizations and $\mathbb{R}$-translations, the following hold

(i) Up to subsequence, $\tilde{v}_{n} \rightarrow \tilde{u}_{r}$ in $C_{\text {loc }}^{\infty}(\mathbb{C} \backslash\{0\})$ as $n \rightarrow \infty$.

(ii) There exist sequences $\delta_{n}^{+} \rightarrow 0^{+}, z_{n} \in \mathbb{C}$ and $c_{n} \in \mathbb{R}$ such that, up to subsequence, $\tilde{v}_{n}\left(\delta_{n} \cdot\right)+c_{n} \rightarrow \tilde{u}_{q}$ in $C_{\text {loc }}^{\infty}(\mathbb{C})$ as $n \rightarrow \infty$.

Proof. After a holomorphic reparametrization of $\tilde{v}_{n}$ and $\mathbb{R}$ translation, we can assume $\left\{\tilde{v}_{n}\right\}$ is a germinating sequence as defined in (2.13)-(2.16). Indeed, we can take sequences $z_{n} \in \mathbb{C}$ and $0<\delta_{n}$, such that $b_{n}\left(z_{n}\right)=\inf b_{n}(\mathbb{C})$ and $\int_{\mathbb{C} \backslash B_{\delta_{n}\left(z_{n}\right)}} v_{n}^{*} d \lambda=\sigma\left(T_{3}\right)$, and define

$$
\tilde{u}_{n}(z)=\left(a_{n}(z), u_{n}(z)\right)=\left(b_{n}\left(z_{n}+\delta_{n} z\right)-b_{n}\left(z_{n}+2 \delta_{n}\right), v_{n}\left(z_{n}+\delta_{n} z\right)\right) .
$$

Then $a_{n}(2)=0, \forall n$ and

$$
\int_{\mathbb{C} \backslash \mathbb{D}} u_{n}^{*} d \lambda=\int_{\mathbb{C} \backslash B_{\delta_{n}}\left(z_{n}\right)} v_{n}^{*} d \lambda=\sigma\left(T_{3}\right) .
$$

\footnotetext{
${ }^{1}$ An open book decomposition of a 3 -manifold $M$ is a pair $(L, p)$, where $L \subset M$ is a link and $p: M \backslash L \rightarrow S^{1}$ is a fibration such that each fiber $p^{-1}(\theta)$ is the interior of a compact embedded surface $S_{\theta} \hookrightarrow M$ satisfying $\partial S_{\theta}=L . L$ is called the binding and the fibers are called pages. If the pages are disks we say that $(L, p)$ has disk-like pages.
} 
Hence, changing the notation back to $\tilde{v}_{n}$, we can assume $\left\{\tilde{v}_{n}\right\}$ is a germinating sequence.

Let $\tilde{u}_{r}: \mathbb{C} \backslash \Gamma_{r} \rightarrow \mathbb{R} \times S^{3}$ be a limit of $\tilde{v}_{n}$ and let $\mathcal{B}=(\mathcal{T}, \mathcal{U})$ be the bubbling-off tree obtained as in Theorem 2.31.

We claim that $P_{3}$ is the asymptotic limit of $\tilde{u}_{r}$ at $\infty$. Indeed, let $\mathcal{W} \subset$ $C^{\infty}\left(S^{1}, S^{3}\right)$ be an $S^{1}$-invariant neighborhood of the set of periodic orbits $P=$ $(x, T) \in \mathcal{P}(\lambda)$ with $T \leq T_{3}$, viewed as maps $x_{T}: S^{1} \rightarrow S^{3}, x_{T}(t)=x(T t)$, such that each connected component of $\mathcal{W}$ contains at most one periodic orbit modulo $S^{1}$-reparametrizations. Using the normalization condition (4.3), we can apply Lemma 2.29 and find $R_{0}>1$ such that for $R \geq R_{0}$, the loops $t \mapsto u_{r}\left(R e^{i 2 \pi t}\right)$ and $\left\{t \mapsto v_{n}\left(R e^{i 2 \pi t}\right)\right\}, n \in \mathbb{N}$ belong to $\mathcal{W}$. For $R$ fixed, the sequence of loops $t \mapsto v_{n}\left(R e^{i 2 \pi t}\right)$ converges to $t \mapsto u_{r}\left(R e^{i 2 \pi t}\right)$ in $C^{\infty}\left(S^{1}, S^{3}\right)$, so that for $n$ large and $R \geq R_{0}, t \mapsto u_{r}\left(R e^{i 2 \pi t}\right)$ and $t \mapsto v_{n}\left(R e^{i 2 \pi t}\right)$ belong to the same connected component of $\mathcal{W}$. This implies that $P_{3}$ is the asymptotic limit of $\tilde{u}_{r}$ at $\infty$.

We first note that $\# \Gamma_{r} \neq 0$. Indeed, if $\Gamma_{r}=\emptyset$, then $\tilde{u}_{r}: \mathbb{C} \rightarrow \mathbb{R} \times S^{3}$ would satisfy the hypotheses of Theorem 4.1, which contradicts the fact that the family $\left\{\tilde{v}_{\tau}\right\}$ is maximal.

Since $P_{3}$ is simply covered, we know that $\tilde{u}_{r}$ is somewhere injective. By Theorem 2.20, we have the estimate

$$
0 \leq \operatorname{ind}\left(\tilde{u}_{r}\right)=3-\sum_{z \in \Gamma_{r}} \mu\left(P_{z}\right)-2+\# \Gamma_{r}+1 .
$$

By Lemma 2.37, $\mu\left(P_{z}\right) \geq 2, \forall z \in \Gamma_{r}$. Hence,

$$
2 \# \Gamma_{r} \leq \sum_{z \in \Gamma_{r}} \mu\left(P_{z}\right) \leq 2+\# \Gamma_{r}
$$

which implies that $\# \Gamma_{r} \leq 2$.

Now we prove that $\# \Gamma_{r}=1$. Suppose, by contradiction, that $\# \Gamma_{r}=2$. Since $\tilde{u}_{r}$ is somewhere injective, we can use Theorem 2.20 to obtain the estimate

$$
0 \leq \operatorname{ind}\left(\tilde{u}_{r}\right)=\mu\left(P_{3}\right)-\sum_{z \in \Gamma_{r}} \mu\left(P_{z}\right)-2+\# \Gamma_{r}+1=4-\sum_{z \in \Gamma_{r}} \mu\left(P_{z}\right) .
$$

Since $\mu\left(P_{z}\right) \geq 2$ for all $z \in \Gamma_{r}$, the only possibility is $\mu\left(P_{z}\right)=2$ for all $z \in \Gamma_{r}$ and ind $\left(\tilde{u}_{r}\right)=0$. By Theorem 2.20, this implies that $\int_{\mathbb{C} \backslash \Gamma_{r}} u_{r}^{*} d \lambda=0$. By Theorem 2.6, there exists a non-constant polynomial $p: \mathbb{C} \rightarrow \mathbb{C}$ and a Reeb orbit $P=(x, T)$ such that $p^{-1}(0)=\Gamma_{r}$ and $\tilde{u}_{r}=F_{P} \circ p$, where $F_{P}: \mathbb{C} \backslash\{0\} \rightarrow \mathbb{R} \times M$ is defined by $F_{P}\left(z=e^{2 \pi(s+i t)}\right)=(T s, x(T t))$, and 
the asymptotic limit of $\tilde{u}_{r}$ at $\infty$ is $P^{\operatorname{deg} p}=(x, \operatorname{deg} p \cdot T)$. Since $P_{3}$ is simply covered, $\operatorname{deg} p=1$. This implies that $\# p^{-1}(0)=1$, a contradiction. Thus we have proved that $\# \Gamma_{r}=1$. Since $b_{n}(0)=\inf b_{n}(\mathbb{C})$ and the puncture in $\Gamma_{r}$ is negative, we have $\Gamma_{r}=\{0\}$.

Now we show that $\int_{\mathbb{C} \backslash\{0\}} u_{r}^{*} d \lambda>0$. Suppose $\pi \cdot d u_{r} \equiv 0$. Then, by Theorem 2.6, there exists a polynomial $p: \mathbb{C} \rightarrow \mathbb{C}$ and an orbit $P=(x, T)$ such that $\Gamma^{\prime}=p^{-1}(0)$ and $\tilde{v}=F_{P} \circ p$, where $F_{P}: \mathbb{C} \backslash\{0\} \rightarrow \mathbb{R} \times S^{3}$ is defined by $F_{P}\left(\zeta=e^{2 \pi(s+i t)}\right)=(T s, x(T t))$. Since 0 is the only root of $p$, we have $p(\zeta)=A \zeta^{n}$, for some $A \neq 0$ and $n \geq 1$. Since $P_{3}$ is prime, we have $n=1$ and $P=P_{3}$. Thus,

$$
\tilde{u}_{r}\left(\zeta=e^{2 \pi(s+i t)}\right)=F_{P_{3}}\left(A e^{2 \pi(s+i t)}\right)=\left(T_{3}\left(s+\frac{\log A}{2 \pi}\right), x_{3}\left(T_{3} t\right)\right) .
$$

and we have the contradiction

$$
T_{3}=\int_{\partial \mathbb{D}} u_{r}^{*} \lambda=\lim _{n \rightarrow \infty} \int_{\mathbb{D}} v_{n}^{*} d \lambda=T_{3}-\sigma(C) .
$$

Here we have used 4.3. We conclude that $\int_{\mathbb{C} \backslash\{0\}} u_{r}^{*} d \lambda>0$.

By Theorem 2.20, we have

$$
1 \leq \operatorname{ind}\left(\tilde{u}_{r}\right)=3-\mu\left(P_{0}\right)-2+\# \Gamma_{r}+1=3-\mu\left(P_{0}\right),
$$

where $P_{0}$ is the asymptotic limit of $\tilde{u}_{r}$ at 0 . Hence $\mu\left(P_{0}\right)=2$.

Following the arguments of Section 2.3.4, we find sequences $\delta_{n} \rightarrow 0^{+}$and $z_{n} \rightarrow z_{0}$ such that the sequence

$$
\tilde{w}_{n}:=\left(b\left(z_{n}+\delta_{n} \cdot\right)-b_{n}\left(z_{n}+2 \delta_{n}\right), v_{n}\left(z_{n}+2 \delta_{n} \cdot\right)\right)
$$

is a germinating sequence. Let $\tilde{u}_{q}=\left(a_{q}, u_{q}\right): \mathbb{C} \backslash \Gamma_{q} \rightarrow \mathbb{R} \times S^{3}$ be a limit of the sequence $\tilde{w}_{n}$. By Lemma 2.39, all asymptotic limits associated to the punctures of $\tilde{u}_{q}$ have Conley-Zehnder index equal to 2. By Lemma 3.20 this implies that $\tilde{u}_{q}$ is somewhere injective. Then, we can apply Theorem 2.20 to $\tilde{u}_{q}$ and obtain

$$
0 \leq \operatorname{ind}\left(\tilde{u}_{q}\right)=2-2 \# \Gamma_{q}-2+\# \Gamma_{q}+1 .
$$

Hence $\# \Gamma_{q} \leq 1$. By Proposition 2.30, we have $\int_{\mathbb{C} \backslash \Gamma_{q}} u_{q}^{*} d \lambda>0$. Again by Theorem 2.20, we have $\operatorname{ind}\left(\tilde{u}_{q}\right) \geq 1$ and consequently $\# \Gamma_{q}=0$. By Proposition 2.38, we conclude that the asymptotic limit of $\tilde{u}_{q}$ at $\infty$ is an orbit $P_{0}$ satisfying $\mu\left(P_{0}\right)=2$ not linked to $P_{3}$. Since the period of $P_{0}$ is less 
than $T_{3}$, by the hypotheses of Theorem 3.5 , we have

$$
P_{0}=P_{2}
$$

and $\tilde{u}_{q}$ is a plane asymptotic to $P_{2}$.

By Lemma 2.34. $\operatorname{wind}_{\pi}\left(\tilde{u}_{r}\right)=0$ and $\operatorname{wind}_{\pi}\left(\tilde{u}_{q}\right)=0$. It follows that the projections $u_{r}: \mathbb{C} \backslash\{0\} \rightarrow S^{3}$ and $u_{q}: \mathbb{C} \rightarrow S^{3}$ are immersions transverse to the Reeb flow.

Proposition 4.2 is still valid if we consider a sequence $\left\{v_{\tau_{n}}\right\}, \tau_{n} \rightarrow 1^{-}$. In this case, denote by $\tilde{u}_{r}^{\prime}: \mathbb{C} \backslash\{0\} \rightarrow \mathbb{R} \times S^{3}$ and $\tilde{u}_{q}^{\prime}: \mathbb{C} \rightarrow \mathbb{R} \times S^{3}$ respectively the finite energy $\tilde{J}$-homolorphic cylinder and the finite energy $\tilde{J}$-homolorphic plane obtained as the SFT-limit of a subsequence of $\left\{v_{\tau_{n}}\right\}$.

Proposition 4.3. $\quad$ (i) For any $S^{1}$-invariant neighborhood $\mathcal{W}_{3}$ of the loop $t \mapsto x_{3}\left(T_{3} t\right)$ in $C^{\infty}\left(\mathbb{R} / \mathbb{Z}, S^{3}\right)$, there exists $R_{0}>>1$ such that for $R \geq$ $R_{0}$, the loop $t \mapsto v_{n}\left(R e^{2 \pi i t}\right)$ belongs to $\mathcal{W}_{3}$.

(ii) For any $S^{1}$-invariant neighborhood $\mathcal{W}_{2}$ of the loop $t \mapsto x_{2}\left(T_{2} t\right)$ in $C^{\infty}\left(\mathbb{R} / \mathbb{Z}, S^{3}\right)$, there exist $\epsilon_{1}>0$ and $R_{1}>>0$ such that the loop $t \mapsto v_{n}\left(z_{n}+R e^{2 \pi i t}\right)$ belongs to $\mathcal{W}$ for $R_{1} \delta_{n} \leq R \leq \epsilon_{1}$.

(iii) Given any neighborhood $\mathcal{V}$ of $u_{r}(\mathbb{C} \backslash\{0\}) \cup u_{q}(\mathbb{C}) \cup P_{2} \cup P_{3}$, we have $v_{\tau_{n}}(\mathbb{C}) \subset \mathcal{V}$ for $n$ large. A similar statement works for any sequence $\tau_{n} \rightarrow 1^{-}$with $u_{q}$ and $u_{r}$ replaced by $u_{q}^{\prime}$ and $u_{r}^{\prime}$ respectively.

Proof. The proof of (i) and (ii) is an application of Lemma 2.29. Let $\mathcal{W}$ be an $S^{1}$-invariant neighborhood of the set of periodic orbits $P=(x, T) \in \mathcal{P}(\lambda)$ with $T \leq T_{3}$, viewed as maps $x_{T}: S^{1} \rightarrow S^{3}, x_{T}(t)=x(T t)$, such that each of the connected components of $\mathcal{W}$ contains at most one periodic orbit modulo $S^{1}$-reparametrizations and such that $\mathcal{W}_{2}, \mathcal{W}_{3} \subset \mathcal{W}$. Using the normalization condition (4.3) we can apply Lemma 2.29 and find $R_{0}>>1$ such that for $R \geq R_{0}$, the loops $t \mapsto u_{r}\left(R e^{i 2 \pi t}\right)$ and $\left\{t \mapsto v_{n}\left(R e^{i 2 \pi t}\right)\right\}, n \in \mathbb{N}$ belong to $\mathcal{W}$. By the asymptotic behavior of the planes $\tilde{v}_{n}$, we know that for each $n$, the loop $t \mapsto v_{n}\left(R e^{s+i t}\right)$ belongs to $\mathcal{W}_{3}$ for $R$ large enough. We conclude that for any $R \geq R_{0}$ and $n$ large, the loop $t \mapsto v_{n}\left(R e^{2 \pi i t}\right)$ belongs to $\mathcal{W}_{3}$.

Let $z_{n} \rightarrow 0$ and $\delta_{n} \rightarrow 0$ be the sequences obtained by soft rescaling near $z=0$ as in the proof of Proposition 4.2, such that passing to a subsequence, we have

$$
\tilde{v}_{n}\left(z_{n}+\delta_{n} \cdot\right)-b_{n}\left(z_{n}+2 \delta_{n}\right) \rightarrow \tilde{u}_{q}
$$

in $C_{\text {loc }}^{\infty}(\mathbb{C})$, as $n \rightarrow+\infty$. Applying Lemma 2.29 as in the proof of Proposition 2.28, we find $\epsilon_{1}>0$ small and $R_{1}>>1$ such that for every $R$ satisfying $\delta_{n} R_{1} \leq R \leq \epsilon_{1}$ and $n$ large, the loop $t \mapsto v_{n}\left(z_{n}+R e^{2 \pi i t}\right)$ belongs to $\mathcal{W}_{2}$. 
The proof of (iii) is a consequence of (i), (ii) and the fact that $v_{n}$ converges uniformly to $u_{r}$ on compact subsets of $\mathbb{C} \backslash\{0\}$ and $w_{n}=v_{n}\left(z_{n}+\delta_{n} \cdot\right)$ converges uniformly to $u_{q}$ on compact subsets of $\mathbb{C}$.

\section{The projected curves are embeddings}

Proposition 4.4. The curves $u_{r}(\mathbb{C} \backslash\{0\}), u_{r}^{\prime}(\mathbb{C} \backslash\{0\}), u_{q}(\mathbb{C})$ and $u_{q}^{\prime}(\mathbb{C})$ do not intersect $P_{2} \cup P_{3}$.

Proof. We first prove that $u_{r}(\mathbb{C} \backslash\{0\}) \cap P_{3}=\emptyset$. Consider $F: \mathbb{C} \backslash\{0\} \rightarrow \mathbb{R} \times S^{3}$ defined by $F\left(e^{2 \pi(s+i t)}\right)=\left(T s, x_{3}(T t)\right)$. Note that $F$ is a finite energy $\tilde{J}_{-}$ holomorphic immersion. Define

$$
A=\left\{(z, \zeta) \in \mathbb{C} \backslash\{0\} \times \mathbb{C} \backslash\{0\} \mid \tilde{u}_{r}(z)=F(\zeta)\right\} .
$$

If the set $A$ is not empty, it consists of isolated points. Indeed, assume that $\left(z^{*}, \zeta^{*}\right)$ is not an isolated point of $A$. Since both $\tilde{u}_{r}$ and $F$ are immersions, we can use Lemma 2.4.3 of MS04 to find open neighborhoods $\mathcal{O}$ and $\mathcal{O}^{\prime}$ of $z^{*}$ and $\zeta^{*}$ respectively, and a holomorphic diffeomorphism $f: \mathcal{O} \rightarrow \mathcal{O}^{\prime}$ such that $F \circ f=\tilde{u}_{r}$ on $\mathcal{O}$. Since $u_{r}$ is transversal to the Reeb flow, we get a contradiction. Now assume $A \neq \emptyset$ and choose $\left(z^{*}, \zeta^{*}\right) \in A$. The maps $\tilde{u}_{r}$ and $F$ intersect transversally at the pair $\left(z^{*}, \zeta^{*}\right)$. By positivity and stability of intersections of pseudo-holomorphic immersions, we find $z_{j} \rightarrow z^{*}$ and $n_{j} \rightarrow \infty$ such that $\tilde{v}_{n_{j}}\left(z_{j}\right) \in F(\mathbb{C} \backslash\{0\})$. This contradicts the fact that $v_{n_{j}}(\mathbb{C}) \cap x_{3}(\mathbb{R})=\emptyset, \forall j$. So we have proved that $A=\emptyset$ and consequently $u_{r}(\mathbb{C} \backslash\{0\}) \cap P_{3}=\emptyset$.

To prove that $u_{r}(\mathbb{C} \backslash\{0\}) \cap P_{2}=\emptyset$, we proceed in the same way, noting that $v_{n}(\mathbb{C}) \cap x_{2}(\mathbb{R})=\emptyset, \forall n$. Indeed, by assumption, $P_{2}$ and $P_{3}$ are not linked. Then the linking number $\operatorname{lk}\left(x_{2}(\mathbb{R}), x_{3}(\mathbb{R})\right)$ is zero. Since each $v_{n}(\mathbb{C})$ is a Seifert surface for $x_{3}(\mathbb{R}), \operatorname{lk}\left(x_{2}(\mathbb{R}), x_{3}(\mathbb{R})\right)$ is the intersection number of $x_{2}(\mathbb{R})$ and $v_{n}(\mathbb{C})$. If $P_{2} \cap v_{n}(\mathbb{C}) \neq \emptyset$, there are positive and negative intersections. But this contradicts the fact that $v_{n}$ is transverse to $R_{\lambda}$.

By the same arguments above, we prove that $u_{r}^{\prime}(\mathbb{C} \backslash\{0\}), u_{q}(\mathbb{C})$ and $u_{q}^{\prime}(\mathbb{C})$ do not intersect $P_{2} \cup P_{3}$.

Using Proposition 4.4 and Theorem B.5, we conclude the following.

Proposition 4.5. The projected curves $u_{r}: \mathbb{C} \backslash\{0\} \rightarrow S^{3}, u_{r}^{\prime}: \mathbb{C} \backslash\{0\} \rightarrow S^{3}$, $u_{q}: \mathbb{C} \rightarrow S^{3}$ and $u_{q}^{\prime}: \mathbb{C} \rightarrow S^{3}$ are embeddings which do not intersect any of their asymptotic limits. 


\section{Uniqueness}

Definition 4.6 ([Sie11]). Let $\tilde{u}: S \backslash \Gamma_{u} \rightarrow \mathbb{R} \times S^{3}$ and $\tilde{v}: S \backslash \Gamma_{v} \rightarrow \mathbb{R} \times S^{3}$ be finite energy $\tilde{J}$-holomorphic curves asymptotic to the same nondegenerate periodic orbit $P \in \mathcal{P}(\lambda)$ at certain punctures $z_{u} \in \Gamma_{u}$ and $z_{v} \in \Gamma_{v}$. We say that $\tilde{u}$ and $\tilde{v}$ approach $P$ in the same direction at these punctures if $\eta_{u}=c \eta_{v}$ for $c>0$, where $\eta_{u}$ and $\eta_{v}$ are the asymptotic eigensections of $\tilde{u}$ at $z_{u}$ and of $\tilde{v}$ at $z_{v}$ respectively, defined in Theorem 2.11. In case $\eta_{u}=c \eta_{v}$, with $c<0$, we say that $\tilde{u}$ and $\tilde{v}$ approach $P$ in opposite directions.

Proposition 4.7. Up to reparametrization and $\mathbb{R}$-translation, $\tilde{u}_{q}=\tilde{u}_{q}^{\prime}$, and $\tilde{u}_{q}$ is the unique finite energy $\tilde{J}$-holomorphic plane asymptotic to $P_{2}$. Also, if $u_{r}(\mathbb{C} \backslash\{0\}) \neq u_{r}^{\prime}(\mathbb{C} \backslash\{0\})$, then (up to reparametrization and $\mathbb{R}$-translation) $\tilde{u}_{r}: \mathbb{C} \backslash\{0\} \rightarrow \mathbb{R} \times S^{3}$ and $\tilde{u}_{r}^{\prime}: \mathbb{C} \backslash\{0\} \rightarrow \mathbb{R} \times S^{3}$ are the unique finite energy $\tilde{J}$-holomorphic cylinders asymptotic to $P_{3}$ at its positive puncture and to $P_{2}$ at its negative puncture that do not intersect $P_{2} \cup P_{3}$. Moreover, $\tilde{u}_{r}$ and $\tilde{u}_{r}^{\prime}$ approach $P_{2}$ in opposite directions.

Proof. The proof follows the ideas of [dPSa18, Proposition C.1].

First we prove that $\tilde{u}_{q}$ and $\tilde{u}_{q}^{\prime}$ coincide up to reparametrization and $\mathbb{R}$ translation. Suppose that $\tilde{u}_{q}$ and $\tilde{u}_{q}^{\prime}$ do not coincide. This is equivalent to $u_{q}(\mathbb{C}) \neq u_{q}^{\prime}(\mathbb{C})$. Since $P_{2}$ is unknotted, $\mu\left(P_{2}\right)=2$ and $\pi_{2}\left(S^{3}\right)=0$, we have from Theorem 1.3 in [HWZ95b] that

$$
u_{q}(\mathbb{C}) \cap P_{2}=\emptyset, \quad u_{q}^{\prime}(\mathbb{C}) \cap P_{2}=\emptyset
$$

and $u_{q}, u_{q}^{\prime}$ are embeddings. By Theorem 1.4 in [HWZ95b], $u_{q}(\mathbb{C}) \neq u_{q}^{\prime}(\mathbb{C})$ and $u_{q}(\mathbb{C}), u_{q}^{\prime}(\mathbb{C}) \cap P_{2}=\emptyset$ imply

$$
u_{q}(\mathbb{C}) \cap u_{q}^{\prime}(\mathbb{C})=\emptyset
$$

Let $\sigma$ be the asymptotic eigensection of $\tilde{u}_{q}$ at $\infty$ and $\sigma^{\prime}$ the asymptotic eigensection of $\tilde{u}_{q}^{\prime}$ at $\infty$. Since by Lemma 2.34 wind $\sigma=$ wind $\sigma^{\prime}=1$ and $\nu_{P_{2}}^{n e g}$ is the only negative eigenvalue of $A_{P_{2}}$ with winding number equal to 1 , it follows that $\sigma, \sigma^{\prime}$ are $\nu_{P_{2}}^{n e g}$-eigensections. Since the eigenspace of $\nu_{P_{2}}^{n e g}$ eigensections is one dimensional, we find a constant $c \neq 0$ such that

$$
\sigma=c \sigma^{\prime}
$$

Assume $c>0$. Since $u_{q}(\mathbb{C}) \cap u_{q}^{\prime}(\mathbb{C})=\emptyset$, by Theorem B.4 we conclude that $\left[\tilde{u}_{q}\right] *\left[\tilde{u}_{q}^{\prime}\right]>0$. Since $u_{q}(\mathbb{C}), u_{q}^{\prime}(\mathbb{C})$ does not intersect $P_{2}$, the orbit $P_{2}$ is even and

$d_{0}\left(\tilde{u}_{q}, \infty\right):=\operatorname{wind}\left(\nu_{P_{2}}^{n e g}\right)-\operatorname{wind}(\sigma)=0, \quad d_{0}\left(\tilde{u}_{q}^{\prime}, \infty\right):=\operatorname{wind}\left(\nu_{P_{2}}^{n e g}\right)-\operatorname{wind}\left(\sigma^{\prime}\right)=0$, 
then we obtain, using Theorem B.1,

$$
\left[\tilde{u}_{q}\right] *\left[\tilde{u}_{q}^{\prime}\right]=0
$$

This contradiction shows that the only possibility is $c<0$. This implies that $u_{q}(\mathbb{C}) \cup u_{q}^{\prime}(\mathbb{C}) \cup x_{2}(\mathbb{R})$ form a $C^{1}$-embedded sphere, where each hemisphere is a strong transverse section, a contradiction with the hypotheses of Theorem 3.5. We have proved that $\tilde{u}_{q}$ and $\tilde{u}_{q}^{\prime}$ coincide up to reparametrization and $\mathbb{R}$-translation. By the same arguments, we prove that any finite energy $\tilde{J}$-holomorphic plane asymptotic to $P_{2}$ coincides with $\tilde{u}_{q}$ up to reparametrization and $\mathbb{R}$-translation.

Now we prove the assertions about the cylinders $\tilde{u}_{r}$ and $\tilde{u}_{r}^{\prime}$. Assume $u_{r}(\mathbb{C} \backslash\{0\}) \neq u_{r}^{\prime}(\mathbb{C} \backslash\{0\})$. Following Theorem 2.11, let $\eta_{+}$be the asymptotic eigensection of $\tilde{u}_{r}$ at $+\infty$ and let $\eta_{-}$be the asymptotic eigensection of $\tilde{u}_{r}$ at 0 . Similarly, define $\eta_{+}^{\prime}$ and $\eta_{-}^{\prime}$ for $\tilde{u}_{r}^{\prime}$. Denote by wind $\left(\eta_{ \pm}\right)$, wind $\left(\eta_{ \pm}^{\prime}\right)$ the winding numbers of $\eta_{ \pm}, \eta_{ \pm}^{\prime}$ computed with respect to a global trivialization of $\xi$. By Lemma 2.34, wind $\left(\eta_{-}\right)=\operatorname{wind}\left(\eta_{-}^{\prime}\right)=1$. Also, $\eta_{-}$and $\eta_{-}^{\prime}$ are associated to the eigenvalue $\nu_{P_{2}}^{\text {pos }}$ defined in (1.13), since there is just one positive eigenvalue of the asymptotic operator $A_{P_{2}}$ with winding number equal to 1 . Since the eigenspace of $\nu_{P_{2}}^{\text {pos }}$ is one dimensional, we conclude that there exists a nonzero constant $c$ so that $\eta_{-}^{\prime}=c \eta_{-}$.

Assume $c>0$, that is, $\tilde{u}_{r}$ and $\tilde{u}_{r}^{\prime}$ approach $P_{2}$ in the same direction. Since we assume $u_{r}(\mathbb{C} \backslash\{0\}) \neq u_{r}^{\prime}(\mathbb{C} \backslash\{0\})$, it follows, by Carleman's similarity principle, that the images of $u_{r}$ and $u_{r}^{\prime}$ do not coincide in any neighborhood of 0 . By Theorem B.4, this implies that $\left[\tilde{u}_{r}\right] *\left[\tilde{u}_{r}^{\prime}\right]>0$. Since $u_{r}(\mathbb{C} \backslash\{0\})$ and $u_{r}^{\prime}(\mathbb{C} \backslash\{0\})$ do not intersect $P_{2} \cup P_{3}$, the orbit $P_{2}$ is even,

$d_{0}\left(\tilde{u}_{r}, 0\right):=\operatorname{wind}\left(\nu_{P_{3}}^{\text {pos }}\right)-\operatorname{wind}\left(\eta_{+}\right), d_{0}\left(\tilde{u}_{r}, \infty\right):=\operatorname{wind}\left(\nu_{P_{2}}^{n e g}\right)-\operatorname{wind}\left(\eta_{-}\right)=0$

and similarly, $d_{0}\left(\tilde{u}_{r}^{\prime}, 0\right)=d_{0}\left(\tilde{u}_{r}^{\prime}, \infty\right)=0$, then, by Theorem B.1, we obtain $\left[\tilde{u}_{r}\right] *\left[\tilde{u}_{r}^{\prime}\right]=0$. This contradiction shows that $c<0$, that is, $\tilde{u}_{r}$ and $\tilde{u}_{r}^{\prime}$ approach $P_{2}$ in opposite directions.

By the same arguments, we conclude that $\tilde{u}_{r}$ and $\tilde{u}_{r}^{\prime}$ are the unique cylinders with the properties given in the statement.

The following proposition can be proved by Proposition 4.4 and Theorem B.2.

Proposition 4.8. The images of the projected curves $u_{r}, u_{q}$ and $\left\{v_{\tau}\right\}, \tau \in$ $(0,1)$ are mutually disjoint. Assume $u_{r}(\mathbb{C} \backslash\{0\}) \neq u_{r}^{\prime}(\mathbb{C} \backslash\{0\})$. Then the images of $u_{r}, u_{r}^{\prime}, u_{q}$ and $\left\{v_{\tau}\right\}, \tau \in(0,1)$ are mutually disjoint. 


\section{The foliation}

Proposition 4.9. The cylinders $u_{r}$ and $u_{r}^{\prime}$ satisfy $u_{r}(\mathbb{C} \backslash\{0\}) \cap u_{r}^{\prime}(\mathbb{C} \backslash\{0\})=$ $\emptyset$. The union of the image of the family $\left\{v_{\tau}: \mathbb{C} \rightarrow S^{3}\right\}, \tau \in(0,1)$ with the images of $u_{q}, u_{r}, u_{r}^{\prime}, x_{2}$ and $x_{3}$ determine singular foliation of a closed region $\mathcal{R}_{1}$ such that $\partial \mathcal{R}_{1}=T$, where $T=x_{2}(\mathbb{R}) \cup x_{3}(\mathbb{R}) \cup u_{r}(\mathbb{C} \backslash\{0\}) \cup u_{r}^{\prime}(\mathbb{C} \backslash\{0\})$.

Proof. First we prove that $u_{r}(\mathbb{C} \backslash\{0\}) \cap u_{r}^{\prime}(\mathbb{C} \backslash\{0\})=\emptyset$. Suppose by contradiction that $u_{r}(\mathbb{C} \backslash\{0\})=u_{r}(\mathbb{C} \backslash\{0\})$. We will show that every point

$$
p \in S^{3} \backslash\left(u_{r}(\mathbb{C} \backslash\{0\}) \cup u_{q}(\mathbb{C}) \cup x_{2}(\mathbb{R}) \cup x_{3}(\mathbb{R})\right)
$$

lies in the image of the family $\left\{v_{\tau}\right\}$. Let

$$
p \in S^{3} \backslash\left(u_{r}(\mathbb{C} \backslash\{0\}) \cup u_{q}(\mathbb{C}) \cup x_{2}(\mathbb{R}) \cup x_{3}(\mathbb{R})\right)
$$

and consider a neighborhood $\mathcal{V}$ of $u_{r}(\mathbb{C} \backslash\{0\}) \cup u_{q}(\mathbb{C}) \cup x_{2}(\mathbb{R}) \cup x_{3}(\mathbb{R})$ such that $p \notin \mathcal{V}$. By Proposition 4.3, we have $v_{\tau_{0}}(\mathbb{C}), v_{\sigma_{0}}(\mathbb{C}) \subset \mathcal{V}$ for some $\tau_{0}$ sufficiently close to 0 and $\sigma_{0}$ sufficiently close to 1 . The surface $S=v_{\tau_{0}}(\mathbb{C}) \cup$ $v_{\sigma_{0}}(\mathbb{C}) \cup x_{3}(\mathbb{R})$ is a piecewise smooth embedded sphere. By Jordan-Brouwer separation theorem, $S$ divides $S^{3}$ into two regions $A_{1}$ and $A_{2}$ with boundary $S$ and disjoint interior. One of these regions, say $A_{1}$ contains $p$ and the other contains $u_{r}(\mathbb{C} \backslash\{0\}) \cup u_{q}(\mathbb{C}) \cup x_{2}(\mathbb{R}) \cup x_{3}(\mathbb{R})$. The intersection of the image of the family $\left\{v_{\tau}\right\}_{\tau \in(0,1)}$ with $A_{1}$ is non empty, open and closed. Thus $p$ is in the image of the family $\left\{v_{\tau}\right\}_{\tau \in(0,1)}$.

It follows that $S^{3}=u_{r}(\mathbb{C} \backslash\{0\}) \cup u_{q}(\mathbb{C}) \cup x_{2}(\mathbb{R}) \cup x_{3}(\mathbb{R}) \cup\left\{v_{\tau}(\mathbb{C})\right\}_{\tau \in(0,1)}$. But this contradicts the fact that $P_{1}$ is not linked to $P_{3}$ and the curves $u_{r}, u_{q}$ and $v_{\tau}$ are transverse to the Reeb flow. We conclude that

$$
u_{r}(\mathbb{C} \backslash\{0\}) \cap u_{r}^{\prime}(\mathbb{C} \backslash\{0\})=\emptyset .
$$

The surface $T=x_{2}(\mathbb{R}) \cup x_{3}(\mathbb{R}) \cup u_{r}(\mathbb{C} \backslash\{0\}) \cup u_{r}^{\prime}(\mathbb{C} \backslash\{0\})$ is a piecewise smooth embedded torus. By Jordan-Brouwer separation theorem, $T$ divides $S^{3}$ into two closed regions $\mathcal{R}_{1}$ and $\mathcal{R}_{2}$. Only one of the regions, say $\mathcal{R}_{1}$ contains the image of the family $\left\{v_{\tau}\right\}$ and the plane $u_{q}(\mathbb{C})$. Now we show that the images of $u_{q}, u_{r}, u_{r}^{\prime}, x_{2}, x_{3}$ and $\left\{v_{\tau}\right\}$ foliate the closed region $\mathcal{R}_{1}$. Let

$$
p \in \mathcal{R}_{1} \backslash\left(x_{2}(\mathbb{R}) \cup x_{3}(\mathbb{R}) \cup u_{r}(\mathbb{C} \backslash\{0\}) \cup u_{r}^{\prime}(\mathbb{C} \backslash\{0\}) \cup u_{q}(\mathbb{C})\right)
$$

and let $\mathcal{V}$ be a neighborhood of $x_{2}(\mathbb{R}) \cup x_{3}(\mathbb{R}) \cup u_{r}(\mathbb{C} \backslash\{0\}) \cup u_{r}^{\prime}(\mathbb{C} \backslash\{0\}) \cup u_{q}(\mathbb{C})$ such that $p \notin \mathcal{V}$. By Proposition 4.3, we have $v_{\tau_{0}}(\mathbb{C}), v_{\sigma_{0}}(\mathbb{C}) \subset \mathcal{V}$ for some $\tau_{0}$ sufficiently close to 0 and $\sigma_{0}$ sufficiently close to 1 . The surface $S=$ 


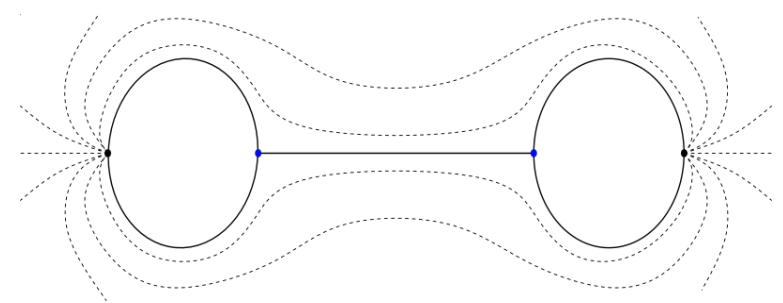

Figure 4.2: The foliation obtained so far

$v_{\tau_{0}}(\mathbb{C}) \cup v_{\sigma_{0}}(\mathbb{C}) \cup x_{3}(\mathbb{R})$ is a piecewise smooth embedded sphere. By JordanBrouwer separation theorem, $S$ divides $S^{3}$ into two regions $A_{1}$ and $A_{2}$ with boundary $S$ and disjoint interiors. One of these regions, say $A_{1}$ contains $p$ and is contained in $\mathcal{R}_{1}$. Thus the intersection of the image of the family $\left\{v_{\tau}\right\}$ with $A_{1}$ is open, closed and non empty. This implies that $p$ is in the image of $v_{\tau}$, for some $\tau \in(0,1)$.

Proposition 4.10. The closure of the image of the family $\left\{v_{\tau}: \mathbb{C} \rightarrow S^{3}\right\}$, that is, the union of the image of the family with the images of $u_{q}, u_{r}, u_{r}^{\prime}, x_{2}$ and $x_{3}$ is homeomorphic to a solid torus.

Proof. We follow the proof of the Solid torus theorem in [Rol03]. Consider the (piecewise smooth) embedded torus $T=x_{2}(\mathbb{R}) \cup x_{3}(\mathbb{R}) \cup u_{r}(\mathbb{C} \backslash\{0\}) \cup$ $u_{r}^{\prime}(\mathbb{C} \backslash\{0\})$. Let $\mathcal{R}_{1}$ be the union of the image of the family of planes $\left\{v_{\tau}\right\}$ with the image of $u_{q}$ and $T$, so that $\partial \mathcal{R}_{1}=T$. The curve $x_{2}(\mathbb{R})$ is the boundary of an embedded disk $\mathcal{D}$ contained in $\mathcal{R}_{1}$ such that $\mathcal{D}^{\circ} \subset \mathcal{R}_{1}$. Let $N$ be a bicollar neighborhood of $\mathcal{D}$ in $\mathcal{R}_{1}$, so that $N \cap T$ is an annular neighborhood of $x_{2}(\mathbb{R})$ in $T$. The boundary of $\mathcal{R}_{1} \backslash N$ is the union of two disks on $\partial N$ and the set $T \backslash N$, which is an annulus. Thus $\mathcal{R}_{1} \backslash N$ is bounded by a piecewise smooth 2-sphere in $S^{3}$. By the generalized Schönflies theorem, $\mathcal{R}_{1} \backslash N$ is homeomorphic to a closed 3-ball. It follows that $\mathcal{R}_{1}$ is homeomorphic to a 3-ball with a $D^{2} \times[0,1]$ attached (by mapping $D^{2} \times\{0\}$ and $D^{2} \times\{1\}$ onto disjoint disks on the boundary of the 3 -ball). Since $\mathcal{R}_{1}$ is orientable, $\mathcal{R}_{1}$ is homeomorphic to $D^{2} \times S^{1}$.

\subsection{Foliating a solid torus in the case $\Gamma_{1}=\{0\}$}

Let

$$
\tilde{v}=(b, v): \mathbb{C} \backslash \Gamma_{1} \rightarrow \mathbb{R} \times S^{3}
$$



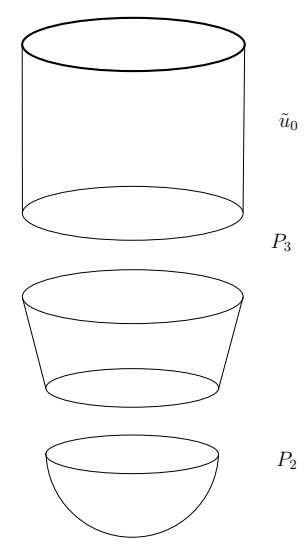

Figure 4.3: Case $\pi \cdot d u_{0} \equiv 0$ and $\Gamma_{1} \neq \emptyset$

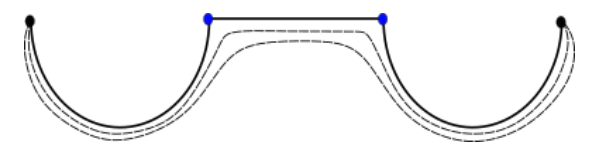

Figure 4.4: Gluing theorem $\Rightarrow$ family of planes

be a limit of the germinating sequence $\tilde{v}_{n}$, as defined in (3.8). In this section, we assume $\Gamma_{1}=\{0\}$. In this case,

$$
\tilde{v}: \mathbb{C} \backslash\{0\} \rightarrow \mathbb{R} \times S^{3}
$$

is a finite energy cylinder asymptotic to $P_{3}$ at its positive puncture and to $P_{2}$ at its negative puncture. The unique vertex immediately below $\tilde{v}$ in the bubbling-off tree $\mathcal{B}$ associated with the germinating sequence $\tilde{v}_{n}$ and the limit $\tilde{v}$ is a plane

$$
\tilde{w}: \mathbb{C} \rightarrow \mathbb{R} \times S^{3}
$$

asymptotic to $P_{2}$.

A family of planes asymptotic to $\boldsymbol{P}_{\mathbf{3}} \quad$ By the Gluing Theorem 5.2 and Theorem 4.1, we obtain a maximal 1-parameter family of planes asymptotic to the orbit $P_{3}$

$$
\tilde{v}_{\tau}=\left(b_{\tau}, v_{\tau}\right), \quad \tau \in\left(\tau_{-}, \tau_{+}\right)
$$

such that $\tilde{v}_{\tau}$ converges, in the SFT sense, to the broken curve $(\tilde{v}, \tilde{w})$ as $\tau \rightarrow \tau_{+}$. Also, both the $\tilde{J}$-holomorphic planes $\tilde{v}_{\tau}$ and the projections $v_{\tau}$ : $\mathbb{C} \rightarrow S^{3}$ are embeddings. This is a consequence of the following Theorem of HWZ95b.

Theorem 4.11 ([HWZ95b, Theorem 1.3]). Consider $S^{3}$ equipped with a tight 
contact form $\lambda$. Assume $\tilde{u}=(a, u): \mathbb{C} \rightarrow \mathbb{R} \times S^{3}$ is a finite energy plane asymptotic to a simply covered and unknotted orbit $P$. If $\mu(P) \leq 3$, then $u(\mathbb{C}) \cap P=\emptyset$ and $u: \mathbb{C} \rightarrow S^{3} \backslash P$ is an embedding.

By the same arguments used in Section 4.1, we conclude that $\tilde{v}_{\tau}$ converges, as $\tau \rightarrow \tau_{-}$, to a broken curve $\left(\tilde{v}^{\prime}, \tilde{w}\right)$, where $\tilde{v}^{\prime}: \mathbb{C} \backslash\{0\} \rightarrow \mathbb{R} \times S^{3}$ is a cylinder asymptotic to $P_{3}$ at its positive puncture $\infty$ and to $P_{2}$ at its negative puncture 0 such that $v^{\prime}(\mathbb{C} \backslash\{0\}) \cap v(\mathbb{C} \backslash\{0\})=\emptyset$. By Proposition 4.10, the union of the image of the family $\left\{v_{\tau}\right\}$ with the images of $v, v^{\prime}, w, x_{2}$ and $x_{3}$ is a solid torus.

\subsection{A cylinder asymptotic to $P_{2}$ and $P_{1}$}

In this section, we use a symplectic cobordism to find a 1-parameter family of generalized pseudoholomorphic planes asymptotic to $P_{2}$, which 'breaks' and produces a pseudoholomorphic cylinder asymptotic to $P_{2}$ at its positive puncture and to $P_{1}$ at its negative puncture.

Symplectic cobordism Following [HWZ98] we define a symplectic cobordism between $\left(S^{3}, \lambda=f \lambda_{0}\right)$ and $\left(S^{3}, \lambda_{E}\right)$, where $\lambda_{E}$ is a dynamically convex contact form on $S^{3}$. Given $0<r_{1}<r_{2}$, with $\frac{r_{1}^{2}}{r_{2}^{2}}$ irrational, let $\lambda_{E}=f_{E} \lambda_{0}$ be the contact form associated to the ellipsoid

$$
E=\left\{\left(x_{1}, y_{1}, x_{2}, y_{2}\right) \in \mathbb{R}^{4} \mid \frac{x_{1}^{2}+y_{1}^{2}}{r_{1}^{2}}+\frac{x_{2}^{2}+y_{2}^{2}}{r_{2}^{2}}=1\right\}
$$

that is, $f_{E}(x, y)=\left(\frac{x_{1}^{2}+y_{1}^{2}}{r_{1}^{2}}+\frac{x_{2}^{2}+y_{2}^{2}}{r_{2}^{2}}\right)^{-1}$.

The Reeb vector field $X_{E}$ defined by $\lambda_{E}$ has precisely two simply covered periodic orbits $\bar{P}_{0}$ and $\bar{P}_{1}$. Both periodic orbits and its iterates are nondegenerate. Their Conley-Zehnder indices are $\mu\left(\bar{P}_{0}\right)=3$ and $\mu\left(\bar{P}_{1}\right)=2 k+1 \geq 5$ where $k \geq 2$ is determined by $k<1+\left(\frac{r_{1}^{2}}{r_{2}^{2}}\right)<k+1$. See [HWZ95a, Lemma 1.6] for a proof of these facts.

We choose $0<r_{1}<r_{2}$ small enough so that

$$
f_{E}<f \text { pointwise on } S^{3}
$$

and a smooth function $h: \mathbb{R} \times S^{3} \rightarrow \mathbb{R}^{+}$satisfying

$$
\begin{aligned}
& h(a, \cdot)=f_{E}, \text { if } a \leq-2, \\
& h(a, \cdot)=f, \text { if } a \geq 2,
\end{aligned}
$$




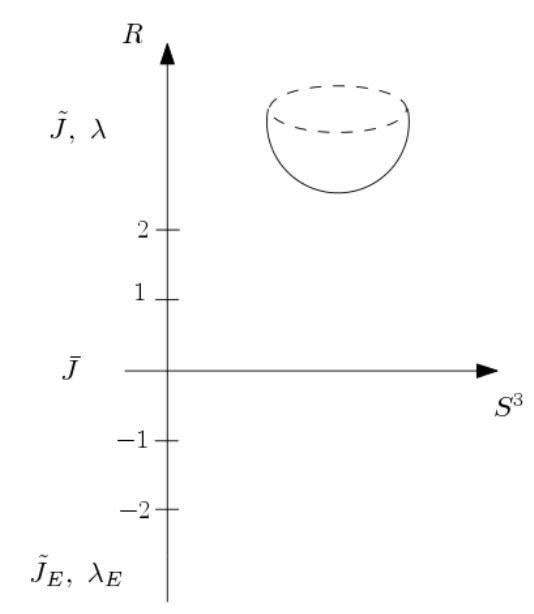

Figure 4.5: The almost complex structure $\bar{J}$

$$
\frac{\partial h}{\partial a} \geq 0 \text { on } \mathbb{R} \times S^{3} \text { and } \frac{\partial h}{\partial a}>\sigma>0 \text { on }[-1,1] \times S^{3} .
$$

In view of 4.5$)$, the 2 -form $d\left(h \lambda_{0}\right)$ restricted to $[-1,1]$ is a symplectic form.

We consider the family of contact forms $\left\{\lambda_{a}=h(a, \cdot) \lambda_{0}, a \in \mathbb{R}\right\}$. The contact structure $\xi=\operatorname{ker} \lambda_{a}$ does not depend on $a$. Choose $J_{E} \in \mathcal{J}\left(\xi, d \lambda_{E}\right)$ and let $\left\{J_{a} \in \mathcal{J}\left(\xi, d \lambda_{a}\right), a \in \mathbb{R}\right\}$ be a smooth family of $d \lambda_{a}$-compatible complex structures on $\xi$ so that $J_{a}=J$ if $a \geq 2$ and $J_{a}=J_{E}$ if $a \leq-2$. We consider smooth almost complex structures $\bar{J}$ on the symplectization $\mathbb{R} \times S^{3}$ with the following properties. On $(\mathbb{R} \backslash[-1,1]) \times S^{3}$, we consider

$$
\left.\bar{J}\right|_{\xi}=J_{a} \text { and } \bar{J} \partial_{a}=X_{\lambda_{a}} .
$$

On $[-1,1] \times S^{3}$ we only require $\bar{J}$ to be compatible with the symplectic form $d\left(h \lambda_{0}\right)$. The space of such almost complex structures on $\mathbb{R} \times S^{3}$ is non-empty and contractible in the $C^{\infty}$-topology and will be denoted by $\mathcal{J}\left(\lambda, J, \lambda_{E}, J_{E}\right)$.

\section{Generalized finite energy surfaces}

Definition 4.12. Let $(S, j)$ be a closed Riemann surface and let $\Gamma \subset S$ be a non empty finite set. A smooth map $\tilde{u}: S \backslash \Gamma \rightarrow \mathbb{R} \times S^{3}$ is called a generalized finite energy surface if it is $\bar{J}$-holomorphic, that is, satisfies $d \tilde{u} \circ j=\bar{J}(\tilde{u}) \circ d \tilde{u}$, for some $\bar{J} \in \mathcal{J}\left(\lambda, J, \lambda_{E}, J_{E}\right)$, as well as the energy condition

$$
0<E(\tilde{u})<+\infty
$$

where the energy is defined as follows. Let $\Sigma$ be the collection of smooth 
functions $\phi: \mathbb{R} \rightarrow[0,1]$ satisfying $\phi^{\prime} \geq 0$ and $\phi=\frac{1}{2}$ on $[-1,1]$. Then

$$
E(\tilde{u})=\sup _{\phi \in \Sigma} \int_{S \backslash \Gamma} \tilde{u}^{*} d(\phi \lambda)
$$

Theorem 2.20 is still valid for almost complex structures in $\mathcal{J}\left(\lambda, J, \lambda_{E}, J_{E}\right)$.

Theorem 4.13 ([HWZ99b] $)$. There exists a residual set $\mathcal{J}_{\text {reg }}^{E} \subset \mathcal{J}\left(\lambda, J, \lambda_{E}, J_{E}\right)$ such that if $\tilde{u}=(a, u): S^{2} \backslash \Gamma \rightarrow \mathbb{R} \times S^{3}$ is a somewhere injective generalized finite energy sphere for $\bar{J} \in \mathcal{J}_{\text {reg }}^{E}$, then

$$
0 \leq \operatorname{ind}(\tilde{u})=\mu(\tilde{u})-\chi_{S^{2}}+\# \Gamma .
$$

We are interested in the space of all finite energy generalized $\bar{J}$-holomorphic planes asymptotic to the orbit $P_{2}$, for fixed $\bar{J} \in \mathcal{J}_{\text {reg }}^{E}$.

The following theorem is a consequence of results in [HWZ99b].

Theorem 4.14 ([HWZ99b]). Let $\tilde{u}_{0}: \mathbb{C} \rightarrow \mathbb{R} \times S^{3}$ be an embedded finite energy $\bar{J}$-holomorphic plane, asymptotic to a non degenerate, simply covered orbit $P=(x, T)$ satisfying $\mu(P)=2$. Then there exists a smooth embedding

$$
\tilde{\Phi}: \mathbb{C} \times(-\epsilon, \epsilon) \rightarrow \mathbb{R} \times S^{3}
$$

with the following properties:

- $\tilde{\Phi}(\cdot, 0)=\tilde{u}_{0}$;

- For every $\tau \in(-\epsilon, \epsilon)$, the map $z \mapsto \tilde{\Phi}(z, \tau)$ is a generalized finite energy $\bar{J}$-holomorphic plane asymptotic to $P$;

- If $\tilde{u}_{n}$ is a sequence of finite energy $\bar{J}$-holomorphic planes asymptotic to $P$ satisfying $\tilde{u}_{n} \rightarrow \tilde{u}_{0}$ in $C_{\text {loc }}^{\infty}(\mathbb{C})$ as $n \rightarrow+\infty$, then there exist sequences $A_{n}, B_{n}$ in $\mathbb{C}$ with $A_{n} \rightarrow 1, B_{n} \rightarrow 0$ and $\tau_{n}$ in $(-\epsilon, \epsilon)$ with $\tau_{n} \rightarrow 0$ such that

$$
\tilde{u}_{n}(z)=\tilde{\Phi}\left(A_{n} z+B_{n}, \tau_{n}\right)
$$

for sufficiently large $n$.

A family of $\bar{J}$-holomorphic planes asymptotic to $P_{2}$ From now on we fix $\bar{J} \in \mathcal{J}_{\text {reg }}^{E}$, where $\mathcal{J}_{\text {reg }}^{E}$ is given by Theorem 4.13 .

Let $\Theta$ be the space of generalized finite energy $\bar{J}$-holomorphic planes asymptotic to $P_{2}$, modulo holomorphic reparametrizations. By Theorem 4.14. $\Theta$ is a smooth 1-dimensional manifold.

Lemma 4.15. The space $\Theta$ is non-empty. 
Proof. Let $\tilde{u}_{q}=\left(a_{q}, u_{q}\right): \mathbb{C} \rightarrow \mathbb{R} \times S^{3}$ be the $\tilde{J}$-holomorphic plane asymptotic to $P_{2}$ given by Theorem 4.2. After a $\mathbb{R}$-translation we can assume $\min _{z \in \mathbb{C}} a_{q}(z)>2$, so that $\tilde{u}_{q}=\left(a_{q}, u_{q}\right)$ can be viewed as a generalized finite energy $\bar{J}$-holomorphic plane asymptotic to $P_{2}$.

By the proof of Lemma 4.15, we have $\tilde{u}_{q} \in \Theta$. Let $\Theta^{\prime}$ be the connected component of $\Theta$ containing $\tilde{u}_{q}$.

\subsubsection{Limiting behavior}

Definition 4.16 (Bubbling-off tree). Consider a finite, rooted and oriented (away from the root) tree $\mathcal{T}$, and a finite set $\mathcal{U}$ of (generalized) finite energy pseudoholomorphic spheres. The pair $\mathcal{B}=(\mathcal{T}, \mathcal{U})$ is called a bubbling-off tree if satisfy the following properties

- There is a bijective correspondence between vertices $q \in \mathcal{T}$ and finiteenergy punctured spheres $\tilde{u}_{q}: \mathbb{C} \backslash \Gamma_{q} \rightarrow \mathbb{R} \times S^{3} \in \mathcal{U}$. Each $\tilde{u}_{\tilde{\tau}_{\tilde{J}}}$ : $\mathbb{C} \backslash \Gamma_{q} \rightarrow \mathbb{R} \times S^{3}$ is pseudoholomorphic with respect to either $\tilde{J}, \tilde{J}_{E}$ or $\bar{J}$. Moreover, each ordered path $\left(q_{1}, \ldots, q_{N}\right)$ from the root $q_{1}=r$ to a leaf $q_{N}$, where $q_{k+1}$ is a direct descendant of $q_{k}$, contains at most one vertex $q_{i}$ such that $\tilde{u}_{q_{i}}$ is $\bar{J}$-holomorphic, in which case $\tilde{u}_{q_{j}}$ is $\tilde{J}_{-}$ holomorphic $\forall 1 \leq j<i$, and $\tilde{u}_{q_{j}}$ is $\tilde{J}_{E}$-holomorphic $\forall i<j \leq N$.

- Each sphere $\tilde{u}_{q}$ has exactly one positive puncture at $\infty$ and $0 \leq \# \Gamma_{q}<$ $\infty$ negative punctures, where $\Gamma_{q}$ is the set of negative punctures of $\tilde{u}_{q}$.

- If the vertex $q$ is not the root then $q$ has an incoming edge $e$ from a vertex $q^{\prime}$, and $\# \Gamma_{q}$ outgoing edges $f_{1}, \ldots, f_{\# \Gamma_{q}}$ to vertices $p_{1}, \ldots, p_{\# \Gamma_{q}}$ of $\mathcal{T}$, respectively. The edge $e$ is associated to the positive puncture of $\tilde{u}_{q}$ and the edges $f_{1}, \ldots, f_{\# \Gamma_{q}}$ are associated to the negative punctures of $\tilde{u}_{q}$. The asymptotic limit of $\tilde{u}_{q}$ at its positive puncture coincides with the asymptotic limit of $\tilde{u}_{q^{\prime}}$ at its negative puncture associated to $e$. In the same way, the asymptotic limit of $\tilde{u}_{q}$ at a negative puncture corresponding to $f_{i}$ coincides with the asymptotic limit of $\tilde{u}_{p_{i}}$ at its unique positive puncture. If $\tilde{u}_{q}$ is $\tilde{J}$-holomorphic, then $\tilde{u}_{p_{i}}$ is either $\tilde{J}$ or $\bar{J}$-holomorphic. If $\tilde{u}_{q}$ is either $\bar{J}$ or $\tilde{J}_{E}$ holomorphic, then $\tilde{u}_{p_{i}}$ is necessarily $\tilde{J}_{E}$-holomorphic, $\forall i=1, \ldots, \# \Gamma_{q}$.

- If the contact area of $\tilde{u}_{q}$ vanishes and $\tilde{u}_{q}$ is $\tilde{J}$ or $\tilde{J}_{E^{-}}$holomorphic, then $\# \Gamma_{q} \geq 2$.

Consider a sequence $\tilde{v}_{n}=\left(a_{n}, v_{n}\right)$ of generalized finite energy planes representing elements of $\Theta^{\prime}$. The energy $E\left(\tilde{v}_{n}\right)$ is uniformly bounded by $T_{2}$. 
The following statement is a corollary of the SFT compactness theorem of $\mathrm{BEH}^{+} 03$.

Theorem 4.17 ([HS16], Theorem 3.11). Up to a subsequence of $\tilde{v}_{n}$, still denoted by $\tilde{v}_{n}$, there exists a bubbling-off tree $\mathcal{B}=(\mathcal{T}, \mathcal{U})$ with the following properties

- For every vertex $q$ of $\mathcal{T}$ there exist sequences $z_{n}^{q}, \delta_{n}^{q} \in \mathbb{C}$ and $c_{n}^{q} \in \mathbb{R}$ such that

$$
\tilde{v}_{n}\left(z_{n}^{q}+\delta_{n}^{q} \cdot\right)+c_{n}^{q} \rightarrow \tilde{v}_{q}(\cdot) \text { in } C_{\text {loc }}^{\infty}\left(\mathbb{C} \backslash \Gamma_{q}\right) \text { as } n \rightarrow \infty
$$

Here $\tilde{v}+c:=(a+c, v)$, where $\tilde{v}=(a, v)$ and $c \in \mathbb{R}$

- The curve $\tilde{v}_{r}$ is asymptotic to $P_{2}$ at $\infty$ and the asymptotic limits of all curves $\tilde{v}_{q}$ are closed orbits with periods $\leq T_{2}$ of the Reeb flow of either $\lambda$ or $\lambda_{E}$.

Lemma 4.18 ([HS16], Lemma 3.12). Let $z_{n}^{q}, \delta_{n}^{q}, c_{n}^{q}$ be sequences such that (4.7) holds for all vertices $q$ of $\mathcal{T}$. Then we can assume, up to a selection of a subsequence still denoted by $\tilde{u}_{n}$, that one of the three mutually excluding possibilities holds for each vertex $q$.

(I) $c_{n}^{q}$ is bounded, $a_{n}\left(z_{n}+\delta_{n}^{q} \cdot\right)$ is $C_{l o c}^{0}\left(\mathbb{C} \backslash \Gamma_{q}\right)$-bounded and $\tilde{v}_{q}$ is a $\bar{J}$ holomorphic curve;

(II) $c_{n}^{q} \rightarrow-\infty, a_{n}\left(z_{n}^{q}+\delta_{n}^{q} \cdot\right) \rightarrow+\infty$ in $C_{\text {loc }}^{0}\left(\mathbb{C} \backslash \Gamma_{q}\right)$ as $n \rightarrow \infty$ and $\tilde{v}_{q}$ is a $\tilde{J}$-holomorphic curve;

(III) $c_{n}^{q} \rightarrow+\infty, a_{n}\left(z_{n}^{q}+\delta_{n}^{q} \cdot\right) \rightarrow-\infty$ in $C_{\text {loc }}^{0}\left(\mathbb{C} \backslash \Gamma_{q}\right)$ as $n \rightarrow \infty$ and $\tilde{v}_{q}$ is a $\tilde{J}_{E}$-holomorphic curve.

Moreover, if $q$ is a vertex for which (III) holds, then $\tilde{u}_{q}$ is asymptotic at its positive puncture to a closed Reeb orbit having period strictly less than $T_{2}$. In particular, (III) does not hold for the root $r$.

The following lemma is proved using the maximum principle combined with estimates for cylinders with small area (Lemma 2.29).

Lemma 4.19 ([HS16, Lemma 3.13]). Let $z_{n}^{r}, \delta_{n}^{q}, c_{n}^{q}$ be sequences such that (4.7) holds for the root $r$. Then, by Theorem 4.17, $P_{2}=\left(x_{2}, T_{2}\right)$ is the asymptotic limit of $\tilde{u}_{r}$ at the positive puncture $\infty$. For every $\mathbb{R} / \mathbb{Z}$-invariant neighborhood $\mathcal{W}$ of $t \mapsto x_{2}\left(T_{2} t\right)$ in $C^{\infty}\left(\mathbb{R} / \mathbb{Z}, S^{3}\right)$ and for every number $M>$ 0 , there exist $R_{0}>0$ and $n_{0}$ such that if $R>R_{0}$ and $n>n_{0}$, then the loop $t \mapsto v_{n}\left(z_{n}^{r}+\delta_{n}^{r} R e^{i 2 \pi t}\right)$ belongs to $\mathcal{W}$ and $a_{n}^{r}\left(z_{n}^{r}+\delta_{n}^{r} e^{i 2 \pi t}\right)+c_{n}^{r}>M$. 
Let $\Theta^{\prime}$ be the connected component of $\Theta$ containing $\tilde{u}_{q}$. Since $\Theta^{\prime}$ is a connected 1-dimensional manifold without boundary, it is diffeomorphic either to $S^{1}$ or to an open interval. It can not be diffeomorphic to $S^{1}$, since it contains the family $\left\{\left[\left(a_{q}+t, u_{q}\right)\right]\right\}_{t>0}$ of equivalence classes of translations of $\tilde{u}_{q}$. Thus, the family is diffeomorphic to an interval and we can assume $\Theta^{\prime}=\left\{\left[\tilde{v}_{\tau}=\left(b_{\tau}, v_{\tau}\right)\right]\right\}_{\tau \in\left(\tau_{-},+\infty\right)}$, where for $\tau \geq 0, \tilde{v}_{\tau}=\left(a_{q}+\tau, u_{q}\right)$. Consider a sequence $\tilde{v}_{n}:=\tilde{v}_{\tau_{n}}$ satisfying $\tau_{n} \rightarrow \tau_{-}$.

Proposition 4.20. The bubbling-off tree obtained as an SFT-limit of the sequence $\left[\tilde{v}_{n}\right]$, as in Theorem 4.17, is as follows. The tree has vertices $r, q$, where $r$ is the root and $q$ is a leaf and direct descendant of $r$. The root $r$ corresponds to a $\tilde{J}$-holomorphic cylinder $\tilde{v}_{r}: \mathbb{C} \backslash\left\{z_{0}\right\} \rightarrow \mathbb{R} \times S^{3}$ asymptotic to $P_{2}$ at its positive puncture $\infty$ and to $P_{1}$ at its negative puncture. The leaf $q$ corresponds to a $\bar{J}$-holomorphic plane asymptotic to $P_{1}$.

Proof. Let $\mathcal{B}=(\mathcal{T}, \mathcal{U})$ be the bubbling-off three given by Theorem 4.17 and let $\tilde{v}_{r}: \mathbb{C} \backslash \Gamma_{r} \rightarrow \mathbb{R} \times S^{3}$ be the finite energy sphere associated to the root

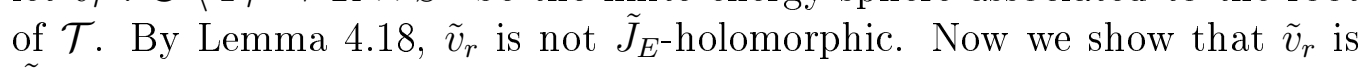
$\tilde{J}$-holomorphic.

Suppose, by contradiction, that $\tilde{v}_{r}$ is $\bar{J}$-holomorphic. By Theorem 4.17 . $\tilde{v}_{r}$ is asymptotic to $P_{2}$ at $\infty$. Since $P_{2}$ is simply covered, $\tilde{v}_{r}$ is somewhere injective. If $\Gamma_{r}=\emptyset$, then $\tilde{v}_{r} \in \Theta$, contradicting the fact that the interval $\Theta^{\prime}$ is maximal. Assume $\Gamma_{r} \neq \emptyset$. By Theorem 4.13, it follows that

$$
0 \leq \operatorname{ind}\left(\tilde{v}_{r}\right)=2-\sum_{z \in \Gamma_{r}} \mu\left(P_{z}\right)-2+\# \Gamma_{r}+1
$$

Then

$$
\sum_{z \in \Gamma_{r}} \mu\left(P_{z}\right)-\# \Gamma_{r} \leq 1
$$

Contradicting the fact that for all $z \in \Gamma_{r}$, the asymptotic limit $P_{z}$ of $\tilde{v}_{r}$ at $z$ is a closed Reeb orbit of $\lambda_{E}$, which is a dynamically convex contact from, that is, $\mu\left(P_{z}\right) \geq 3$, for all $z \in \Gamma_{r}$. Thus, $\tilde{v}_{r}$ is $\tilde{J}_{\text {-holomorphic. }}$

Let $m:=\min a_{q}(\mathbb{C})$. We claim that

$$
\limsup _{n}\left(\min b_{n}(\mathbb{C})\right) \leq m .
$$

To prove the claim, suppose by contradiction that $\lim \sup _{n}\left(\min b_{n}(\mathbb{C})\right)>$ $m>2$. Then there exists a subsequence $\tilde{v}_{n_{k}}=\left(b_{n_{k}}, v_{n_{k}}\right)$ satisfying $\min b_{n_{k}}(\mathbb{C})>$ $m$. By the definiton of $\bar{J}$, the planes $\tilde{v}_{n_{k}}$ are $\tilde{J}$-holomorphic. By the hypotheses of Theorem 3.5. $\tilde{v}_{q}$ is the only $\tilde{J}$-holomorphic plane asymptotic to $P_{2}$, up 
to reparametrization and $\mathbb{R}$-translation. This implies $\left[\tilde{v}_{n_{k}}\right]=\left[a_{q}+\tau_{k}, u_{q}\right]$ for a sequence $\tau_{k}>0$. This contradicts the fact that $\Theta^{\prime}$ is an interval.

Now we show that $\Gamma_{r} \neq \emptyset$. Suppose, by contradiction, that $\tilde{v}_{r}$ is a $\tilde{J}_{-}$ holomorphic plane. Let $z_{n}, \delta_{n}$ and $c_{n}$ be the sequences given by Theorem 4.17, such that

$$
\tilde{v}_{n}\left(z_{n}+\delta_{n} \cdot\right)+c_{n} \rightarrow \tilde{v}_{r}(\cdot) \text { in } C_{l o c}^{\infty}(\mathbb{C}) \text { as } n \rightarrow \infty .
$$

The limit $\tilde{v}_{r}$ satisfies $4.18(\mathrm{II})$, so that $c_{n} \rightarrow-\infty$ and $b_{n}\left(z_{n}+\delta_{n} \cdot\right) \rightarrow+\infty$ in $C_{l o c}^{0}(\mathbb{C})$ as $n \rightarrow \infty$. By Lemma 4.19 , there exists $R_{0}>0$ such that $b_{n}\left(z_{n}+\delta_{n} z\right)>m-c_{n}>m$ for $|z|>R_{0}$ and $n$ large enough. For $|z| \leq R_{0}$, by Lemma 4.18 (II), we have $b_{n}\left(z_{n}+\delta_{n} z\right)>m$, for $n$ large enough. Thus, $\inf b_{n}(\mathbb{C})>m$ for $n$ large enough. This contradicts $\lim \sup _{n} \inf b_{n}(\mathbb{C}) \leq m$, and concludes the proof of $\Gamma_{r} \neq \emptyset$.

So far, we know that $\tilde{v}_{r}: \mathbb{C} \backslash \Gamma_{r} \rightarrow \mathbb{R} \times M$ is a $\tilde{J}$-holomorphic sphere and $\Gamma_{r} \neq \emptyset$. The next step is to prove that every negative asymptotic limit of $\tilde{v}_{r}$ has Conley-Zehnder index equal to 1.

I) If $q$ is not the root and $P_{\infty}$ is the asymptotic limit of $\tilde{v}_{q}$ at $\infty$, then $\mu\left(P_{\infty}\right) \geq 1$.

Suppose, by contradiction, that $\mu\left(P_{\infty}\right) \leq 0$. The curve $\tilde{v}_{q}: \mathbb{C} \backslash \Gamma_{q} \rightarrow$ $\mathbb{R} \times S^{3}$ factors as

$$
\tilde{v}_{q}=\tilde{u} \circ p
$$

where $\tilde{u}: \mathbb{C} \backslash \Gamma^{\prime} \rightarrow \mathbb{R} \times S^{3}$ is a somewhere injective finite energy sphere and $p$ is a polynomial. If $P$ is the asymptotic limit of $\tilde{u}$ at $\infty$, then $P^{\operatorname{deg} p}=P_{\infty}$. By Lemma 1.9, $\mu\left(P_{\infty}\right) \leq 0$ implies $\mu(P) \leq 0$.

By Theorem 4.13 ,

$$
0 \leq \operatorname{ind} \tilde{u}:=\mu(P)-\sum_{z^{\prime} \in \Gamma^{\prime}} \mu\left(P_{z^{\prime}}\right)+\# \Gamma_{q}-1,
$$

where $P_{z^{\prime}}$ is the asymptotic limit of $\tilde{u}$ at $z^{\prime}$.

If $\Gamma^{\prime}=\emptyset$, we already have a contradiction. Otherwise, there exists $z_{0}^{\prime} \in \Gamma^{\prime}$ such that $\mu\left(P_{z_{0}^{\prime}}\right) \leq 0$. Let $z_{0} \in \Gamma_{q}$ be such that $p\left(z_{0}\right)=z_{0}^{\prime}$. Then $P_{z_{0}}=P_{z_{0}^{\prime}}^{k}$, for some $k \leq \operatorname{deg} p$, where $P_{z_{0}}$ is the asymptotic limit of $\tilde{v}_{q}$ at $z_{0}$. By Lemma 1.9 , we have $\mu\left(P_{z_{0}}\right) \leq 0$.

Since the tree has a finite number of vertices, by induction we find a leaf $l$ of the tree such that the finite energy plane $\tilde{u}_{l}: \mathbb{C} \rightarrow \mathbb{R} \times S^{3}$ is asymptotic to an orbit $P$ with $\mu(P) \leq 0$, a contradiction. 
II) For every $z \in \Gamma_{r}$, we have $\mu\left(P_{z}\right)=1$, where $P_{z}$ is the asymptotic limit of $\tilde{v}_{r}$ at $z$.

By Theorem 2.20, we have

$$
0 \leq \operatorname{ind} \tilde{v}_{r}=2-\sum_{z \in \Gamma_{r}} \mu\left(P_{z}\right)+\# \Gamma_{r}-1
$$

It follows that

$$
\sum_{z \in \Gamma_{r}} \mu\left(P_{z}\right) \leq \# \Gamma_{r}+1
$$

Since, by Claim I), we have $1 \leq \mu\left(P_{z}\right)$, for every $z \in \Gamma_{r}$, then there exists at most one puncture $z_{0} \in \Gamma_{r}$ such that $\mu\left(P_{z_{0}}\right) \geq 2$. If there exists such puncture, we have ind $\tilde{v}_{r}=0$. By Theorem 2.20, this implies $\pi \circ d v_{r} \equiv 0$. By the definition of bubbling-off tree (Definition 4.16), this implies $\# \Gamma_{r} \geq 2$. By Theorem 2.6, there exists a periodic orbit $P$ and a polynomial $p: \mathbb{C} \rightarrow \mathbb{C}$ such that $p^{-1}(0)=\Gamma_{r}$ and $\tilde{v}_{r}=F_{P} \circ p$, where $F_{P}$ is the cylinder over the orbit $P$. This contradicts the fact that $P_{2}$ is simply covered.

We conclude that $\mu\left(P_{z}\right)=1$, for all $z \in \Gamma_{r}$. We also proved that $\pi \circ d v_{r} \neq$ 0 .

III) $\quad \tilde{v}_{r}$ is a cylinder asymptotic to $P_{1}$ at its negative puncture.

By Lemma 2.34, we conclude that $\operatorname{wind}_{\infty}\left(\tilde{v}_{r}, z\right)=1, \forall z \in \Gamma_{r} \cup\{\infty\}$. Recall that

$$
u_{q}(\mathbb{C}) \cap x_{2}(\mathbb{R})=\emptyset .
$$

Thus, the curves $\tilde{u}_{q}$ and $\tilde{v}_{r}$ satisfy condition (2) of Theorem B.2. Here $\tilde{u}_{q}$ is the plane asymptotic to $P_{2}$ obtained by Theorem 4.2. It follows from Theorem $\mathrm{B} .2$ that the projected curve $u_{q}$ does not intersect any of the negative asymptotic limits of $\tilde{v}_{r}$. This implies that $P_{2}$ is contractible in $S^{3} \backslash P_{z}$, for every $P_{z}$ asymptotic limit of $\tilde{v}_{r}$ at $z \in \Gamma_{r}$. Consequently,

$$
\operatorname{lk}\left(P_{2}, P_{z}\right)=\operatorname{lk}\left(P_{z}, P_{2}\right)=0, \forall z \in \Gamma_{r} .
$$

Since, by hypothesis, the orbit $P_{1}$ is the only orbit with Conley-Zehnder index 1 and period less than $T_{2}$ that is not linked to $P_{2}$, it follows that

$$
P_{z}=P_{1}, \quad \forall z \in \Gamma_{r} .
$$

Moreover, the hypothesis $T_{2}<2 T_{1}$ implies that $\# \Gamma_{r}=1$.

IV) Now we prove that the next (and last) level of the bubbling-off tree consists of a $\bar{J}$-holomorphic plane. 
Let $\tilde{v}_{q}: \mathbb{C} \backslash \Gamma_{q} \rightarrow \mathbb{R} \times S^{3}$ be the finite energy sphere associated to the only vertex that is a direct descendant of the root $r$. The asymptotic limit of $\tilde{v}_{q}$ at $\infty$ is $P_{1}$, that is a simply covered orbit. It follows that $\tilde{v}_{q}$ is somewhere injective. By the definition of bubbling-off tree, $\tilde{v}_{q}$ is either $\tilde{J}$-holomorphic or $\bar{J}$-holomorphic. By Theorem 4.13 , we have

$$
0 \leq \operatorname{ind} \tilde{v}_{q}=1-\sum_{z \in \Gamma_{q}} \mu\left(P_{z}\right)+\# \Gamma_{q}-1=\# \Gamma_{q}-\sum_{z \in \Gamma_{q}} \mu\left(P_{z}\right)
$$

where $P_{z}$ is the asymptotic limit of $\tilde{v}_{q}$ at $z \in \Gamma_{q}$. Since by Claim I), $\mu\left(P_{z}\right) \geq 1$ for all $z \in \Gamma_{q}$, then ind $\tilde{v}_{q}=0$ and $\mu\left(P_{z}\right)=1$, for all $z \in \Gamma_{q}$.

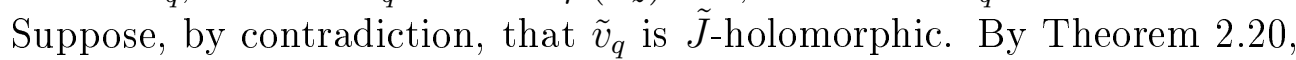
we have $\pi \circ d v_{q} \equiv 0$. By the definition of bubbling-off tree 4.16, this implies $\# \Gamma_{q} \geq 2$. Theorem 2.6 and the fact that $P_{1}$ is simply covered lead to a contradiction. We have proved that $\tilde{v}_{q}$ is $\bar{J}$-holomorphic.

Suppose that $\Gamma_{q} \neq \emptyset$. By the definition of bubbling-off tree, if $l$ is a vertex of the tree that is a direct descendant of $q$, then $\tilde{v}_{l}$ is necessarily $\tilde{J}_{E^{-}}$ holomorphic. The asymptotic limit of $\tilde{v}_{l}$ at $\infty$ is equal to $P_{z}$ for some $z \in \Gamma_{q}$. But $\mu\left(P_{z}\right)=1$ for all $z \in \Gamma_{q}$, contradicting the fact that all closed orbits of $X_{E}$ have Conley-Zehnder index $\geq 3$.

We have proved that $\Gamma_{q}=\emptyset$. This finishes the proof of Proposition 4.20.

Proposition 4.21. The curve $\tilde{v}_{r}=\left(b_{r}, v_{r}\right): \mathbb{C} \backslash\{0\} \rightarrow \mathbb{R} \times S^{3}$ and the projection $v_{r}: \mathbb{C} \backslash\{0\} \rightarrow S^{3}$ are embeddings. The projection $v_{r}$ does not intersect any of its asymptotic limits.

To prove Proposition 4.21, we need the following.

Proposition 4.22. Let $\tilde{u}: \mathbb{R} \times S^{1} \rightarrow \mathbb{R} \times S^{3}$ be a finite energy cylinder asymptotic to prime orbits $P=(x, T)$ at $+\infty$ and $\bar{P}=(\bar{x}, \bar{T})$ at $-\infty$, and satisfying

- $P \neq \bar{P}$,

- $P$ and $\bar{P}$ form an unlink,

- $\mu(P), \mu(\bar{P}) \in\{1,2,3\}$.

Then $u\left(\mathbb{R} \times S^{1}\right) \cap P=\emptyset$ and $u\left(\mathbb{R} \times S^{1}\right) \cap \bar{P}=\emptyset$.

Remark 4.23. In Proposition 4.22, the fact that the contact form $\lambda$ in $S^{3}$ is tight is crucial. 
Proof. The proof follows the arguments of the proof of Theorem 1.3 in HWZ95b, so we just sketch the proof here and refer to the results in [HWZ95b when necessary.

A finite energy surface $\tilde{u}: \mathbb{C} \backslash \Gamma \rightarrow \mathbb{R} \times S^{3}$ such that $\pi \cdot d u$ is not identically zero can intersect its asymptotic limits in at most finitely many points. This is proved in [HWZ96, Theorem 5.2]. This allows the definition of an algebraic intersection index as follows. Take a small embedded 2-disk $\mathcal{D}$ transversal to the periodic orbit at a point $x(t)$ of $P$ and tangent to $\xi=\operatorname{ker} \lambda$ at $x(t)$, that is, $T_{x(t) \mathcal{D}}=\xi_{x(t)}$. We orient the disk in such a way that $T_{x(t) \mathcal{D}}$ and $\xi_{x(t)}$ have the same orientation. Let $M \in \mathbb{R}$ be such that all the intersection points of $u$ with $P$ are contained in $u\left((-\infty, M) \times S^{1}\right)$. Let $\varphi: \mathbb{D} \rightarrow S^{3}$ be a disk map to $\bar{P}$ such that $\varphi(\mathbb{D}) \cap P=\emptyset$. Such disk exists because $P \cup \bar{P}$ is the trivial link. Glue the disk $\mathbb{D}$ to $[-\infty, M] \times S^{1}$ along $-\infty \times S^{1}$ to form a new disk $D$. Let $\bar{u}: \overline{\mathbb{R}} \times S^{1} \rightarrow S^{3}$ be the map obtained by defining $\bar{u}(-\infty, t)=\bar{x}(\bar{T} t)$ and $\bar{u}(+\infty, t)=x(T t)$. Define $U: D \rightarrow S^{3}$ by

$$
\left.U\right|_{[-\infty, M] \times S^{1}}=\bar{u},\left.\quad U\right|_{\mathbb{D}}=\varphi .
$$

Consider $U_{*}: H_{1}(\partial D, \mathbb{Z}) \rightarrow H_{1}\left(S^{3} \backslash P, \mathbb{Z}\right)$. If $\alpha$ is the generator of $H_{1}(\partial D)$, then since $U(\partial D) \cup P=\emptyset$, there exists an integer, that we call $\operatorname{int}(u)$, such that

$$
U_{*}(\alpha)=\operatorname{int}(u)[\partial \mathcal{D}] \in H_{1}\left(S^{3} \backslash P, \mathbb{Z}\right) .
$$

Here we used the fact that $H_{1}\left(S^{3} \backslash P\right)$ is generated by $[\partial \mathcal{D}]$.

It follows from the proof of Theorem 4.6 in [HWZ95b that $\operatorname{int}(u)$ is the oriented intersection number of $U$ with $P$. Also, all the intersections are in the image of the map $u$, since there is no intersections of $P$ with $\varphi(\mathbb{D})$. Following the proof of Theorem 4.6 in [HWZ95b], one can show that $\operatorname{int}(u) \geq 0$, so that $\operatorname{int}(u)=0$ if and only if $u\left(\mathbb{R} \times S^{1}\right) \cup P=\emptyset$. Thus, to show that $u\left(\mathbb{R} \times S^{1}\right) \cap P=\emptyset$, it is sufficient to show that $\operatorname{int}(u)=0$.

Let $\Phi: \mathcal{U} \rightarrow S^{3}$ be an embedding of an open neighborhood $\mathcal{U}$ of the zero section of $\left.\xi\right|_{P}$. We require that $\Phi\left(0_{p}\right)=p$ and the fiberwise derivative of $\Phi$ at $0_{p}$ is the inclusion of $\xi$ into $T_{p} S^{3}$.

Consider a non vanishing section $v(t)$ of $\xi$ along $P$ which is contained in $\mathcal{U}$. Define the loop $\beta(v)$ by $\beta(v)(t)=\Phi \circ v(t)$ for $0 \leq t \leq T$. It is contained in $S^{3} \backslash P$ and we denote by $[\beta(v)] \in H_{1}\left(S^{3} \backslash P\right)$ the homology class generated by this loop. Fix the global trivialization $\Psi: \xi \rightarrow S^{3} \times \mathbb{R}^{2}$. Let wind $(v, \Psi)$ be the winding number of the small section $v(t)$ with respect to the trivialization $\Psi$. It is proved in [HWZ95b] that

$$
\operatorname{wind}(v, \Psi)[\partial \mathcal{D}]-[\beta(v)] \in H_{1}\left(S^{3} \backslash P\right)
$$




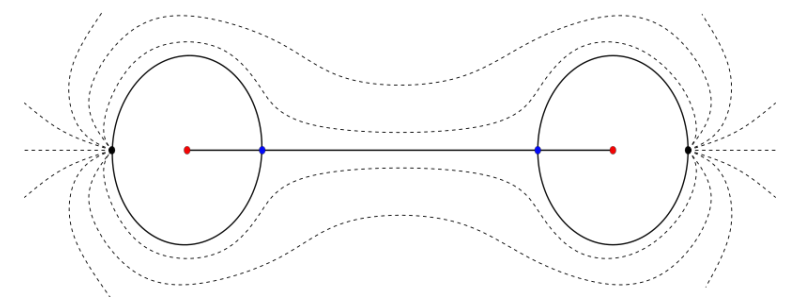

Figure 4.6: The foliation of the region $\mathcal{R}_{1}$ and the cylinder $v_{r}$ connecting the orbits $P_{2}$ and $P_{1}$.

is independent of the section $v$ as described above. We define a constant $c(u)$ by

$$
(\operatorname{wind}(v, \Psi)-c(u))[\partial \mathcal{D}]=[\beta(v)] .
$$

If we choose, for example, the special section $v(t)$ such that $\Phi \circ v(t)=u\left(s^{*}, t\right)$ for some large $s^{*} \in \mathbb{R}$, then by definition

$$
[\beta(v)]=\operatorname{int}(u)[\partial \mathcal{D}]
$$

and taking the limit as $s^{*} \rightarrow \infty$, we have

$$
\operatorname{wind}_{\infty}(\tilde{u}, \infty)-c(u)=\operatorname{int}(u) .
$$

It is proved in [HWZ95b] that there exists an embedded disc $F=\varphi(\mathbb{D})$ with $\varphi(\partial \mathbb{D})=P$ whose characteristic distribution has $e^{+} \geq 1$ positive elliptic points, and that

$$
c(u)=2 e^{+}-1 .
$$

By Lemma 2.34, we have $\operatorname{wind}_{\infty}(\tilde{u}, \infty)=1$, so that

$$
\operatorname{int}(u)=2-2 e^{+} \text {. }
$$

Since $\operatorname{int}(u) \geq 0$ and $e^{+} \geq 1$, then $\operatorname{int}(u)=0$ and $e^{+}=1$. This shows that $u\left(\mathbb{R} \times S^{1}\right) \cap P=\emptyset$. We can repeat the arguments replacing $P$ by $\bar{P}$ and show that $u\left(\mathbb{R} \times S^{1}\right) \cap \bar{P}=\emptyset$ and conclude the proof.

Proof of Proposition 4.21. From Proposition 4.22, we conclude that $\tilde{v}_{r}$ does not intersect its asymptotic limits. Thus $\tilde{v}_{r}$ satisfies condition (3) of Theorem B.5. Applying Theorem B.5, we conclude the proof.

Proposition 4.24. Up to reparametrization, $\tilde{v}_{r}$ is the unique $\tilde{J}$-holomorphic cylinder asymptotic to $P_{2}$ at $\infty$ and to $P_{1}$ at 0 that do not intersect the orbits $P_{1}$ and $P_{2}$. Moreover, the cylinder $\tilde{v}_{r}$ and the plane $\tilde{u}_{q}$ approach $P_{2}$ in opposite directions, according to Definition 4.6. 


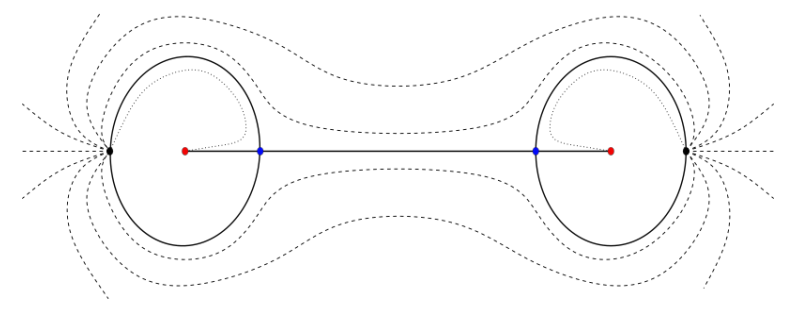

Figure 4.7: Gluing theorem $\Rightarrow$ family of cylinders asymptotic to $P_{3}$ and $P_{1}$

Proof. The proof follows the ideas of [dPSa18, Proposition C.1]. Following Theorem 2.11, let $\eta_{+}$be the asymptotic eigensection of $\tilde{u}_{q}$ at $\infty$ and let $\eta_{+}^{\prime}$ be the asymptotic eigensection of $\tilde{v}_{r}$ at $\infty$. By Lemma 2.34 $\operatorname{wind}_{\infty}\left(\tilde{u}_{q}, \infty\right)=$ wind $\left(\tilde{v}_{r}, \infty\right)=1$. Using $\mu\left(P_{2}\right)=2$, formula (1.15) and Proposition 1.15 . we conclude that $\nu_{P_{2}}^{\text {neg }}$ is the unique negative eigenvalue of $A_{P_{2}}$ with winding number 1. By the properties of the asymptotic operator $A_{P_{2}}$ given in Section 1.3 , we know that the eigenspace of $\nu_{P_{2}}^{\text {neg }}$ is one dimensional. Thus, there exists $c \neq 0$ such that $\eta_{+}^{\prime}=c \eta_{+}$. Suppose $c>0$, that is, $\tilde{v}_{r}$ and $\tilde{u}_{q}$ approach $P_{2}$ in the same direction. By Carleman's similarity principle, the images of $\tilde{v}_{r}$ and $\tilde{u}_{q}$ do not coincide in any neighborhood of $\infty$. By Theorem B.4, we have $\left[\tilde{v}_{r}\right] *\left[\tilde{u}_{q}\right]>0$. On the other hand, we can apply Theorem B.1 to the curves $\tilde{v}_{r}$ and $\tilde{u}_{q}$ to get $\left[\tilde{v}_{r}\right] *\left[\tilde{u}_{q}\right]=0$. With this contradiction, we prove that $\tilde{v}_{r}$ and $\tilde{u}_{q}$ approach $P_{2}$ in opposite directions, that is, $c<0$.

Using the same arguments, one can show that any cylinder with the properties given in the statement must have the same image as $\tilde{v}_{r}$.

\subsection{A family of cylinders asymptotic to $P_{1}$ and $P_{3}$}

So far we have foliated a region $\mathcal{R}_{1} \subset S^{3}$ homeomorphic to a solid torus with boundary equal to the torus $T=P_{3} \cup u_{r}(\mathbb{C} \backslash\{0\}) \cup u_{r}^{\prime}(\mathbb{C} \backslash\{0\}) \cup P_{2}$. The complement of $\mathcal{R}_{1}$ in $S^{3}$ is a closed region that we denote by $\mathcal{R}_{2}$. In the previous section we found an embedded pseudoholomorphic cylinder $\tilde{v}_{r}$ : $\mathbb{C} \backslash\{0\} \rightarrow \mathbb{R} \times S^{3}$. The projected curve $v_{r}$ is also embedded and its image is contained in the interior of $\mathcal{R}_{2}$.

Applying the Gluing Theorem 5.2 to the broken cylinder $\left(\tilde{u}_{r}, \tilde{v}_{r}\right)$, we obtain a family of pseudoholomorphic cylinders $\left\{\tilde{w}_{\tau}: \mathbb{C} \backslash\{0\} \rightarrow \mathbb{R} \times S^{3}\right\}, \tau \in$ $[R,+\infty)$, asymptotic to the orbits $P_{3}$ and $P_{1}$, converging to the broken cylin$\operatorname{der}\left(\tilde{u}_{r}, \tilde{v}_{r}\right)$ in the sense of the SFT compactness theorem as $\tau \rightarrow+\infty$.

Proposition 4.25. For every $\tau \in[R,+\infty), \tilde{w}_{\tau}$ is an embedding, the projec- 
tion $w_{\tau}: \mathbb{C} \backslash\{0\} \rightarrow S^{3}$ is an embedding which does not intersect its asymptotic limits and $w_{\tau}(\mathbb{C} \backslash\{0\}) \subset \mathcal{R}_{2}$.

Proof. By Proposition 4.22 and Theorem B.5. we conclude that $\tilde{w}_{\tau}$ and $w_{\tau}$ are embeddings and $w_{\tau}$ does not intersect its asymptotic limits. Applying Theorem B.2 to $\tilde{w}_{\tau}$ and any $\tilde{J}$-holomorphic plane asymptotic to $P_{3}$ whose projection is in $\mathcal{R}_{1}$, we conclude that $\left.w_{\tau}(\mathbb{C} \backslash\{0\})\right) \subset \mathcal{R}_{2}$.

The following is Theorem 4.5.44 of [Wen05].

Theorem 4.26 ([Wen05]). Let $\tilde{u}=(a, u): S^{2} \backslash \Gamma \rightarrow \mathbb{R} \times M$ be an embedded $\tilde{J}_{-}$ holomorphic finite energy sphere with ind $(\tilde{u})=2$, such that every asymptotic limit is simply covered and has odd Conley-Zehnder index. Then there exists a number $\delta>0$ and an embedding

$$
\begin{aligned}
\tilde{F}: \mathbb{R} \times(-\delta, \delta) \times S^{2} \backslash \Gamma & \rightarrow \mathbb{R} \times M \\
(\sigma, \tau, z) & \mapsto\left(a_{\tau}(z)+\sigma, u_{\tau}(z)\right)
\end{aligned}
$$

such that

- For $\sigma \in \mathbb{R}$ and $\tau \in(-\delta, \delta)$, the maps $\tilde{u}_{(\sigma, \tau)}=\tilde{F}(\sigma, \tau, \cdot)$ are (up to parametrization) embedded $\tilde{J}$-holomorphic finite energy spheres and $\tilde{u}_{(0,0)}=\tilde{u}$.

- The $\operatorname{map} F(\tau, z)=u_{\tau}(z)$ is an embedding $(-\delta, \delta) \times S^{2} \backslash \Gamma \rightarrow M$ and its image never intersects the asymptotic limits. In particular, the maps $u_{\tau}: S^{2} \backslash \Gamma \rightarrow M$ are embedded for each $\tau \in(-\delta, \delta)$ with mutually disjoint images which do not intersect their asymptotic limits.

- For any sequence $\tilde{v}_{k}: S^{2} \backslash \Gamma \rightarrow \mathbb{R} \times M$ such that for each puncture in $\Gamma, \tilde{v}_{k}$ has the same asymptotic limit as $\tilde{u}$, with the same sign, and $\tilde{v}_{k} \rightarrow \tilde{u}$ in $C_{\text {loc }}^{\infty}\left(S^{2} \backslash \Gamma\right)$, there is a sequence $\left(\sigma_{k}, \tau_{k}\right) \rightarrow(0,0) \in \mathbb{R} \times(-\delta, \delta)$ such that $\tilde{v}_{k}=\tilde{u}_{\left(\sigma_{k}, \tau_{k}\right)} \circ \varphi_{k}$ for some sequence of diffeomorphisms $\varphi_{k}$ : $S^{2} \backslash \Gamma \rightarrow S^{2} \backslash \Gamma$ and $k$ sufficiently large.

Remark 4.27. In Theorem 4.26, no genericity assumption is required for $\tilde{J}$.

Applying the theorem above to the maps $\tilde{w}_{\tau}$, we obtain a maximal smooth one parameter family of maps, that we denote again by $\tilde{w}_{\tau}, \tau \in\left(\tau_{-}, \tau_{+}\right)$. We assume the normalization $\tau_{-}=0, \tau_{+}=1$ and $\tilde{w}_{\tau} \rightarrow\left(\tilde{u}_{r}, \tilde{v}_{r}\right)$, as $\tau \rightarrow 0^{+}$.

Proposition 4.28. Consider a sequence $\tilde{w}_{n}=\left(c_{n}, w_{n}\right): \mathbb{C} \backslash\{0\} \rightarrow \mathbb{R} \times S^{3}$, where $\tilde{w}_{n}=\tilde{w}_{\sigma_{n}}$ and $\sigma_{n} \rightarrow 0^{+}$. Then after suitable reparametrizations and $\mathbb{R}$-translations, we have 
(i) Up to subsequence, $\tilde{w}_{n} \rightarrow \tilde{u}_{r}$ in $C_{l o c}^{\infty}(\mathbb{C} \backslash\{0\})$ as $n \rightarrow \infty$.

(ii) There exist sequences $\delta_{n}^{+} \rightarrow 0^{+}$and $d_{n} \in \mathbb{R}$ such that, up to subsequence, $\tilde{w}_{n}\left(\delta_{n} \cdot\right)+d_{n} \rightarrow \tilde{v}_{r}$ in $C_{\text {loc }}^{\infty}(\mathbb{C} \backslash\{0\})$ as $n \rightarrow \infty$.

A similar statement holds for any sequence $\tau_{n} \rightarrow 1^{-}$with $\tilde{u}_{r}$ replaced with $\tilde{u}_{r}^{\prime}$.

The Proof of Proposition 4.28 is the subject of Sections 4.4.1 and 4.4.2.

\subsubsection{Bubbling-off analysis for the family of cylinders}

In this section, we follow the ideas of [HWZ03, Section 6.2].

Consider a sequence $\tilde{w}_{n}=\left(c_{n}, w_{n}\right): \mathbb{C} \backslash\{0\} \rightarrow \mathbb{R} \times S^{3}$, where $\tilde{w}_{n}=\tilde{w}_{\sigma_{n}}$ and $\sigma_{n} \rightarrow 0^{+}$.

Note that since all cylinders $\tilde{w}_{n}$ are asymptotic to $P_{3}$ and $P_{1}$, we have $0<E\left(\tilde{w}_{n}\right) \leq T_{3}$.

We reparametrize the sequence so that

$$
\int_{\mathbb{C} \backslash \mathbb{D}} w_{n}^{*} d \lambda=\frac{\sigma\left(T_{3}\right)}{2} .
$$

Define

$$
\Theta=\left\{z \in \mathbb{C} \backslash\{0\} \mid \exists \text { subsequence } \tilde{w}_{n_{j}} \text { and } z_{j} \rightarrow z \text { s.t. }\left|\nabla \tilde{w}_{n_{j}}\left(z_{j}\right)\right| \rightarrow \infty\right\}
$$

By the same arguments used in the proof of Proposition 2.26, we can assume that $\Theta$ is finite and $\Theta \subset \mathbb{D} \backslash\{0\}$. Also, there exists a $\tilde{J}$-holomorphic map

$$
\tilde{w}: \mathbb{C} \backslash(\{0\} \cup \Theta) \rightarrow \mathbb{R} \times S^{3}
$$

such that, up to a subsequence, still denoted by $\tilde{w}_{n}$,

$$
\tilde{w}_{n} \rightarrow \tilde{w} \text { in } C_{l o c}^{\infty}\left(\mathbb{C} \backslash(\{0\} \cup \Theta), \mathbb{R} \times S^{3}\right)
$$

and $E(\tilde{w}) \leq T_{3}$.

The punctures in $\{0\} \cup \Theta$ are non-removable and negative, and the puncture $z=\infty$ is positive. Indeed for any $\epsilon$ sufficiently large or small, we have

$$
\int_{\partial B_{\epsilon}(0)} w^{*} \lambda=\lim _{n \rightarrow \infty} \int_{\partial B_{\epsilon}(0)} w_{n}^{*} \lambda \in\left[T_{1}, T_{3}\right],
$$

where $\partial B_{\epsilon}(z)$ is oriented counterclockwise. It follows that $\infty$ is a positive puncture and 0 is a negative puncture. If $z \in \Theta$, then for any sufficiently 
small $\epsilon$, we have

$$
\int_{\partial B_{\epsilon}(z)} w^{*} \lambda=\lim _{n \rightarrow \infty} \int_{\partial B_{\epsilon}(z)} w_{n}^{*} \lambda=\lim _{n \rightarrow \infty} \int_{B_{\epsilon}(z)} w_{n}^{*} d \lambda \geq T>0,
$$

where $T \leq T_{3}$ is a period. This follows from the same arguments used in Section 2.3.3. It follows that $z$ is a negative puncture.

By the same arguments used in the proof of Proposition 4.2 we conclude that the asymptotic limit of $\tilde{w}$ at $\infty$ is $P_{3}$.

Lemma 4.29 .

$$
\int_{\mathbb{C} \backslash(\{0\} \cup \Theta)} w^{*} d \lambda>0 .
$$

Proof. If $\Theta=\emptyset$, then it follows from 4.10 that $\int_{\mathbb{C} \backslash\{0\}} w^{*} d \lambda \geq \frac{\sigma\left(T_{3}\right)}{2}$. In the case $\Theta \neq \emptyset$, suppose $\int_{\mathbb{C} \backslash(\{0\} \cup \Theta)} w^{*} d \lambda=0$. By Theorem 2.6, there exists a polynomial $p: \mathbb{C} \rightarrow \mathbb{C}$ and a periodic orbit $P \in \mathcal{P}(\lambda)$ such that $p^{-1}(0)=$ $\{0\} \cup \Theta$ and $\tilde{w}=F_{P} \circ p$, where $F_{P}$ is the cylinder over the orbit $P$. But this implies $\operatorname{deg} p \geq 2$, contradicting the fact that the asymptotic limit of $\tilde{w}$ at $\infty$ is $P_{3}$, that is a prime orbit.

Soft rescaling near $z_{0} \in \Theta$. Assume $\Theta \neq \emptyset$ and take a puncture $z_{0} \in \Theta$. Let $P_{z_{0}}=\left(x_{z_{0}}, T_{z_{0}}\right)$ be the asymptotic limit of $\tilde{w}$ at $z_{0}$. As in Section 2.3.4. we define the mass of $z_{0}$ by

$$
m\left(z_{0}\right)=\lim _{\epsilon \rightarrow 0^{+}} m_{\epsilon}\left(z_{0}\right) \quad m_{\epsilon}\left(z_{0}\right)=\lim _{n \rightarrow \infty} \int_{B_{\epsilon}\left(z_{0}\right)} w_{n}^{*} d \lambda,
$$

where $\partial B_{\epsilon}\left(z_{0}\right)$ is oriented counterclockwise. Now we proceed as in the soft rescaling done in Section 2.3.4. Fix $\epsilon>0$ such that

$$
m_{\epsilon}\left(z_{0}\right)-m\left(z_{0}\right) \leq \frac{\sigma\left(T_{3}\right)}{2} .
$$

Choose $z_{n}$ defined by $c_{n}\left(z_{n}\right)=\inf \left(c_{n}\left(B_{\epsilon}\left(z_{0}\right)\right)\right)$ and $0<\delta_{n}<\epsilon$ by

$$
\int_{B_{\epsilon}\left(z_{0}\right) \backslash B_{\delta_{n}}\left(z_{n}\right)} w_{n}^{*} d \lambda=\sigma\left(T_{3}\right) .
$$

It follows that $z_{n} \rightarrow z_{0}$ and, up to a subsequence, $\delta_{n} \rightarrow 0$. Take $R_{n} \rightarrow \infty$ such that $\delta_{n} R_{n}<\frac{\epsilon}{2}$ and define

$$
\begin{aligned}
\tilde{v}_{n}=\left(b_{n}, v_{n}\right): B_{R_{n}}(0) & \rightarrow \mathbb{R} \times S^{3} \\
z & \mapsto\left(c_{n}\left(z_{n}+\delta_{n} z\right)-c_{n}\left(z_{n}+2 \delta_{n}\right), w_{n}\left(z_{n}+\delta_{n} z\right)\right)
\end{aligned}
$$


The sequence $\tilde{v}_{n}$ is a germinating sequence according to Definition 2.25. Let

$$
\Theta_{1}=\left\{z \in \mathbb{C} \mid \exists z_{j} \rightarrow z \text { and subsequence } \tilde{w}_{n_{j}} \text { s.t. }\left|d \tilde{w}_{n_{j}}\left(z_{j}\right)\right| \rightarrow \infty\right\} \text {. }
$$

Passing to a subsequence, we can assume $\Theta_{1}$ is finite. Let

$$
\tilde{v}=(b, v): \mathbb{C} \backslash \Theta_{1} \rightarrow \mathbb{R} \times S^{3}
$$

be a limit of $\tilde{v}_{n}$ as defined in 2.27. Then $\tilde{v}$ has a unique positive puncture at $\infty$ and $\tilde{v}$ is asymptotic to $P_{0}$ at $\infty$. Thus, using Lemma 2.37, we conclude the following.

Lemma 4.30. If $z \in \Theta$ and $\tilde{w}$ is asymptotic to $P$ at $z$, then $\mu(P) \geq 2$.

Since $P_{3}$ is prime, it follows that $\tilde{w}$ is somewhere injective. By Theorem 2.20, we have

$$
1 \leq \operatorname{ind}(\tilde{w})=3-\sum_{z \in\{0\} \cup \Theta} \mu\left(P_{z}\right)+\# \Theta
$$

and consequently

$$
\sum_{z \in\{0\} \cup \Theta} \mu\left(P_{z}\right) \leq 2+\# \Theta
$$

This proves the following lemma.

Lemma 4.31. Assume $\Theta \neq \emptyset$. Then $\mu\left(P_{0}\right) \leq 1$. If $\mu\left(P_{0}\right)=1$, then $\# \Theta=1$ and $\mu\left(P_{z}\right)=2$, where $P_{z}$ is the asymptotic limit of $\tilde{w}$ at the unique point $z \in \Theta$.

Soft-rescaling near $z=0$. For any $\epsilon>0$, define

$$
m_{\epsilon}(0)=\lim _{n \rightarrow \infty} \int_{B_{\epsilon}(0) \backslash\{0\}} w_{n}^{*} d \lambda,
$$

and define the mass of the puncture $z=0$ by

$$
m(0)=\lim _{\epsilon \searrow 0} m_{\epsilon}(0)
$$

Note that, for $n$ large and $\epsilon$ small, we have

$$
\int_{B_{\epsilon}(0) \backslash\{0\}} w_{n}^{*} d \lambda=\int_{\partial B_{\epsilon}(0)} w_{n}^{*} \lambda-\lim _{\delta \rightarrow 0} \int_{\partial B_{\delta}(0)} w_{n}^{*} \lambda=\int_{\partial B_{\epsilon}(0)} w_{n}^{*} \lambda-T_{1} .
$$


It follows that

$$
m(0)=\lim _{\epsilon \searrow 0} \int_{\partial B_{\epsilon}(0)} w^{*} \lambda-T_{1}=T_{0}-T_{1},
$$

where $T_{0}$ is the period of the asymptotic limit $P_{0}=\left(x_{0}, T_{0}\right)$ of $\tilde{w}$ at the puncture $z=0$. We have two cases:

- either $m(0)=0$ or

- $m(0)>0 \Rightarrow m(0)>\sigma\left(T_{3}\right)$.

I) First assume that $m(0)>\sigma\left(T_{3}\right)>0$. We claim that there is a sequence $\delta_{n} \rightarrow 0$ satisfying

$$
\int_{D_{\delta_{n}(0)} \backslash\{0\}} w_{n}^{*} d \lambda=m(0)-\frac{\sigma(C)}{2}
$$

Indeed, there exists a sequence $\delta_{n}$ satisfying the equation above, since using 4.10) and $\int_{\mathbb{C} \backslash\{0\}} w_{n}^{*} d \lambda=T_{3}-T_{1} \geq m(0)>\sigma(C)$, we conclude

$$
\int_{\mathbb{D} \backslash\{0\}} w_{n}^{*} d \lambda \geq m(0)-\frac{\sigma(C)}{2}>0 .
$$

Now we show that $\lim \inf \delta_{n}=0$, so that, passing to a subsequence, still denoted by $\delta_{n}$, the claim is true. Suppose that there exists $0<\epsilon^{\prime}<\lim \inf \delta_{n}$. Then we have the contradiction

$$
m(0)-\frac{\sigma(C)}{2}=\lim _{j \rightarrow \infty} \int_{B_{\delta_{n}(0) \backslash\{0\}}} w_{n}^{*} d \lambda \geq \lim _{j \rightarrow \infty} \int_{B_{\epsilon}^{\prime}(0) \backslash\{0\}} w_{n}^{*} d \lambda \geq m(0),
$$

and the claim is proved.

Let $\epsilon_{0}>0$ be small enough so that the disks $B_{\epsilon_{0}}(\gamma), \gamma \in \Theta \cup\{0\}$ are disjoint. Define

$$
\tilde{v}_{n}(z)=\left(b_{n}(z), v_{n}(z)\right)=\left(c_{n}\left(\delta_{n} z\right)-c_{n}\left(2 \delta_{n}\right), w_{n}\left(\delta_{n} z\right)\right)
$$

for $z \in B \frac{\epsilon_{0}}{\delta_{n}}(0) \backslash\{0\}$. Note that $\tilde{v}_{n}$ satisfies the normalization

$$
\int_{\mathbb{D} \backslash\{0\}} \tilde{v}_{n}^{*} d \lambda=\int_{B_{\delta_{n}}(0) \backslash\{0\}} w_{n}^{*} d \lambda=m(0)-\frac{\sigma(C)}{2} .
$$


It follows that, for $j$ large and $\epsilon_{0}$ small we have the estimate

$$
\begin{aligned}
\int_{\substack{B \frac{\epsilon_{0}}{\delta_{n} \backslash \mathbb{D}}\\
}} v_{n}^{*} d \lambda & =\int_{\substack{\frac{\epsilon_{0}}{\delta_{n}} \backslash\{0\}\\
}} v_{n}^{*} d \lambda-\int_{\mathbb{D} \backslash\{0\}} v_{n}^{*} d \lambda \\
& =\int_{B_{\epsilon_{0}}(0) \backslash\{0\}} w_{n}^{*} d \lambda-\left(m(0)-\frac{\sigma(C)}{2}\right) \\
& \leq m(0)+\frac{\sigma(C)}{2}-\left(m(0)-\frac{\sigma(C)}{2}\right)=\sigma(C)
\end{aligned}
$$

Define $\Theta_{0}$ as the set of bubbling points of the sequence $\tilde{v}_{n}$. Using the proof of Proposition 2.26 we obtain, passing to a subsequence, $\Theta_{0}$ finite and $\Theta_{0} \subset$ $\mathbb{D} \backslash\{0\}$. Also, there exists a $\tilde{J}$-holomorphic map $\tilde{v}_{0}: \mathbb{C} \backslash\{0\} \cup \Theta_{0} \rightarrow \mathbb{R} \times S^{3}$ such that, passing to a subsequence

$$
\tilde{v}_{n} \rightarrow \tilde{v}_{0} \text { in } C_{\text {loc }}^{\infty}\left(\mathbb{C} \backslash\{0\} \cup \Theta_{0}\right) .
$$

The map $\tilde{v}_{0}$ is non-constant, the punctures in $\{0\} \cup \Theta_{0}$ are non-removable and negative, and the puncture $z=\infty$ is positive.

Lemma 4.32. The asymptotic limit of $\tilde{v}_{0}$ at its unique positive puncture $z=\infty$ is equal to $P_{0}$, the asymptotic limit of $\tilde{w}$ at $\{0\}$.

Proof. Let $\mathcal{W}$ be an open neighborhood of the set of loops

$$
\left\{t \in S^{1} \mapsto x(T t+c) \mid P=(x, T) \in \mathcal{P}(\lambda), c \in \mathbb{R}\right\}
$$

such that each connected component of $\mathcal{W}$ contains at most one periodic orbit modulo $S^{1}$-reparametrization. Let $P_{\infty}$ be the asymptotic limit of $\tilde{v}$ at $\infty$. Let $\mathcal{W}_{\infty}$ and $\mathcal{W}_{0}$ be connected components of $\mathcal{W}$ containing $P_{\infty}$ and $P_{0}$ respectively.

Since $\tilde{w}_{n} \rightarrow \tilde{w}$ in $C_{l o c}^{\infty}$, we can choose $0<\epsilon_{0}^{\prime}<\epsilon_{0}$ small enough so that, if $0<\rho \leq \epsilon_{0}^{\prime}$ is fixed, then the loop

$$
t \in S^{1} \mapsto w_{n}\left(\rho e^{i 2 \pi t}\right)
$$

belongs to $\mathcal{W}_{0}$ for $n$ large. Since $\tilde{v}_{n} \rightarrow \tilde{v}$ in $C_{l o c}^{\infty}$, we can choose $R_{0}>1$ large enough so that, if $R \geq R_{0}$ is fixed, then the loop

$$
t \in S^{1} \mapsto v_{n}\left(R e^{i 2 \pi t}\right)=w_{n}\left(\delta_{n} R e^{i 2 \pi t}\right)
$$

belongs to $\mathcal{W}_{\infty}$ for $n$ large. 
By 4.16, we can show that

$$
e:=\liminf \int_{\partial B_{\delta_{n} R_{0}(0)}} w_{n}^{*} \lambda>0
$$

Consider, for each $n$, the $\tilde{J}$-holomorphic cylinder $\tilde{C}_{n}:\left[\frac{\ln R_{o} \delta_{n}}{2 \pi}, \frac{\ln \epsilon_{0}^{\prime}}{2 \pi}\right] \times S^{1} \rightarrow$ $\mathbb{R} \times S^{3}$, defined by $\tilde{C}_{n}(s, t)=\tilde{w}_{n}\left(e^{2 \pi(s+i t)}\right)$. It follows from 4.18 that

$$
\int\left[\frac{\ln R_{o} \delta_{n}}{2 \pi}, \frac{\ln \epsilon_{0}^{\prime}}{2 \pi}\right] \times S^{1} C_{n}^{*} d \lambda \leq \sigma(C)
$$

for $n$ large. Using (4.19) and 4.20) and applying Lemma 2.29, we find $h>0$ so that the loop

$$
t \mapsto C_{n}(s, t)
$$

belongs to $\mathcal{W}$ for every $s \in\left[\frac{\ln R_{0} \delta_{n}}{2 \pi}+h, \frac{\ln \epsilon_{0}}{2 \pi}-h\right]$ and $n$ large. Since $h>0$, we have

$$
C_{n}\left(\frac{\ln \epsilon_{0}}{2 \pi}-h, t\right)=w_{n}\left(\epsilon_{0} e^{-2 \pi h} e^{2 \pi i t}\right) \in \mathcal{W}_{0}
$$

for all $n$ large and

$$
C_{n}\left(\frac{\ln R_{0} \delta_{n}}{2 \pi}+h, t\right)=w_{n}\left(R_{0} \delta_{n} e^{2 \pi h} e^{2 \pi t}\right) \in \mathcal{W}_{\infty}
$$

for all $n$ large. Thus $\mathcal{W}_{\infty}=\mathcal{W}_{0}$ and $P_{\infty}=P_{0}$.

Lemma 4.33. Either

- $\int_{\mathbb{C} \backslash\{0\} \cup \Theta_{0}} v_{0}^{*} d \lambda>0$ or

- $\int_{\mathbb{C} \backslash\{0\} \cup \Theta_{0}} v_{0}^{*} d \lambda=0$ and $\# \Theta_{0} \geq 1$.

Proof. Indeed, suppose, by contradiction, that $\int_{\mathbb{C} \backslash\{0\} \cup \Theta_{0}} v^{*} d \lambda=0$ and $\Theta_{0}=$ $\emptyset$. Then

$$
\begin{aligned}
T_{0}=\int_{\partial \mathbb{D}} v^{*} \lambda & =\lim _{n \rightarrow \infty} \int_{\partial B_{\delta_{n}}(0)} w_{n}^{*} \lambda \\
& =\int_{B_{\delta_{n}}(0) \backslash\{0\}} w_{n}^{*} \lambda+\lim _{\epsilon \rightarrow 0} \int_{\partial B_{\epsilon}(0)} w_{n}^{*} \lambda \\
& =m(0)-\frac{\sigma\left(T_{3}\right)}{2}+T_{1} \\
& =T_{0}-\frac{\sigma\left(T_{3}\right)}{2}
\end{aligned}
$$

a contradiction. 
II) Now assume $m(0)=0$. Let $\epsilon>0$ be small enough so that $m_{\epsilon}(0) \leq \frac{\sigma\left(T_{3}\right)}{2}$. Define

$$
\tilde{v}_{n}(z)=\tilde{w}_{n}\left(\delta_{n} z\right), \quad z \in \mathbb{C} \backslash\{0\}
$$

for any sequence $\delta_{n} \rightarrow 0$. We have

$$
\int_{B_{\delta_{n}} \backslash B_{\delta_{n}}} v_{n}^{*} d \lambda=\int_{B_{\epsilon} \backslash B_{\delta_{n}^{2}}} w_{n}^{*} d \lambda \leq \int_{B_{\epsilon} \backslash\{0\}} w_{n}^{*} d \lambda .
$$

Thus,

$$
\lim _{n \rightarrow \infty} \int_{B_{\frac{\epsilon}{\delta_{n}}} \backslash B_{\delta_{n}}} v_{n}^{*} d \lambda \leq m_{\epsilon}(0) \leq \frac{\sigma(C)}{2} .
$$

It follows that $\left\{\tilde{v}_{n}\right\}$ has no bubbling points. Ideed, if there is a bubblingoff point, arguing as in the proof of Proposition 2.26, we conclude that $\lim _{n \rightarrow \infty} \int_{B_{\frac{\epsilon}{\delta_{n}}} \backslash B_{\delta_{n}}} v_{n}^{*} d \lambda \geq T$, for some period $T$.

Thus, passing to a subsequence, there exists a $\tilde{J}$-holomorphic map $\tilde{v}_{0}=$ $\left(b_{0}, v_{0}\right): \mathbb{C} \backslash\{0\} \rightarrow \mathbb{R} \times S^{3}$ such that

$$
\tilde{v}_{n} \rightarrow \tilde{v}_{0} \text { in } C_{l o c}^{\infty}(\mathbb{C} \backslash\{0\}) .
$$

It follows from (4.21) that

$$
\int_{\mathbb{C} \backslash\{0\}} v_{0}^{*} d \lambda=0 .
$$

Indeed, if $\int_{\mathbb{C} \backslash\{0\}} v_{0}^{*} d \lambda>0$, then $\int_{\mathbb{C} \backslash\{0\}} v_{0}^{*} d \lambda>\sigma\left(T_{3}\right)$, which contradicts 4.21.

We claim that $\tilde{v}_{0}$ is non-constant. Indeed,

$$
\int_{\partial \mathbb{D}} v_{n}^{*} \lambda=\int_{\partial B_{\delta_{n}}} w_{n}^{*} \lambda=\int_{\partial B_{\epsilon}} w_{n}^{*} \lambda-\int_{B_{\epsilon} \backslash B_{\delta_{n}}} w_{n}^{*} \lambda
$$

Thus,

$$
\int_{\partial \mathbb{D}} v_{0}^{*} \lambda=\lim _{n \rightarrow \infty} \int_{\partial \mathbb{D}} v_{n}^{*} \lambda=\lim _{n \rightarrow \infty}\left(\int_{\partial B_{\epsilon}} w_{n}^{*} \lambda-\int_{B_{\epsilon} \backslash B_{\delta_{n}}} w_{n}^{*} \lambda\right)=\int_{\partial B_{\epsilon}} w^{*} \lambda .
$$

Taking the limit as $\epsilon \rightarrow 0$, we get

$$
\int_{\partial \mathbb{D}} v_{0}^{*} \lambda=T_{0}=T_{1}
$$

It follows that $\tilde{v}_{0}$ is a cylinder over a $T_{0}$-periodic Reeb orbit $P=\left(x, T_{0}\right)$. 
Lemma 4.34. $P_{0}=P_{1}$.

Proof. Choose now an $S^{1}$-invariant neighborhood $\mathcal{W}$ in $C^{\infty}\left(S^{1}, S^{3}\right)$ separating the loops associated with periodic solutions of period $\leq T_{3}$ from each other. For fixed $n$, we know that

$$
\left(t \mapsto w_{n}\left(\epsilon e^{2 \pi i t}\right)\right) \rightarrow x_{1}(T t) \text { as } \epsilon \rightarrow 0
$$

We choose a sequence $\delta_{n} \rightarrow 0$ such that

$$
\left(t \mapsto w_{n}\left(\delta_{n} e^{2 \pi i t}\right)\right) \in \mathcal{W}, \quad \forall n
$$

Recalling that

$$
\left(t \mapsto w\left(\epsilon e^{2 \pi i t}\right)\right) \rightarrow x_{0}(T t) \text { as } \epsilon \rightarrow 0,
$$

We conclude, from estimate 4.21 and Lemma 2.29, arguing as in Section 2.3.4, that $P=P_{1}=P_{0}$.

We conclude the analysis of the case $m(0)=0$.

Going back to the case $m(0)>\sigma\left(T_{3}\right)>0$, if the mass of the puncture $z=0$ of $\tilde{v}_{0}$ is positive or $\Theta_{0} \neq 0$, we repeat the process. It necessarily stops after finitely many iterations, when we reach punctures with zero mass or run out of bubbling-off points. This follows from Lemmas 2.30 and 4.33 . We obtain a tree of finite energy spheres having a unique positive puncture whose asymptotic limit agrees with the asymptotic limit of the corresponding negative puncture belonging to the previous level. The leaves of the tree correspond to finite energy planes originating from the bubbling-off points and a cylinder over the orbit $P_{1}$, originating from the puncture $z=0$.

We are ready to proof Proposition 4.28.

\subsubsection{Proof of Proposition 4.28}

First we show that the mass of the puncture $z=0$, as defined in (4.14), is positive. Suppose $m(0)=0$. Then, by the analysis done in Section 4.4.1. either

1) $\Theta=\emptyset$ and $\tilde{w}: \mathbb{C} \backslash\{0\} \rightarrow \mathbb{R} \times S^{3}$ is asymptotic to $P_{3}$ at $z=\infty$ and asymptotic to $P_{1}$ at $z=0$, or

2) $\Theta=\left\{z_{1}, \ldots, z_{k}\right\}$ and $\tilde{w}: \mathbb{C} \backslash\{0\} \cup \Theta: \mathbb{R} \times S^{3}$ is asymptotic to $P_{3}$ at $z=\infty$, to $P_{1}$ at $z=0$ and to orbits $\tilde{P}_{i}$ satisfying $\mu\left(\tilde{P}_{i}\right) \geq 2$ at each $z_{i}$, $i \in\{1, \ldots, k\}$. 


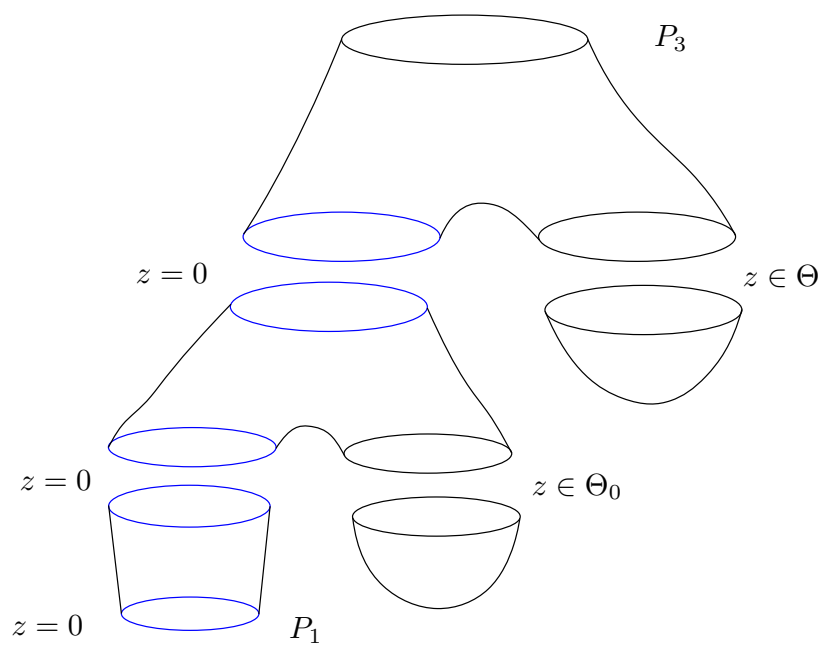

Figure 4.8: An (a priori) possible bubbling-off tree

Option 1) can not occur, since it would contradict the fact that the family of cylinders $\left\{\tilde{w}_{\tau}\right\}$ is maximal.

Now we show that option 2) also leads to a contradiction. Suppose 2) holds. By Lemma 4.31, we have $k=1$ and $\mu(\tilde{P})=2$, where $\tilde{P}=(\tilde{x}, \tilde{T})$ is the asymptotic limit of $\tilde{w}$ at the unique puncture in $\Theta$. The orbit $\tilde{P}$ is not linked to $P_{3}$. This follows from positivity and stability of intersections and the fact that $\tilde{P}$ is the limit of contractible links contained in the image of the embedded cylinders $w_{n}$, which do not intersect $P_{3}$. Since $\tilde{T}<T_{3}$, by the hypotheses of Theorem 3.5 we conclude that $\tilde{P}=P_{2}$. But $\tilde{T}$ also satisfies $T_{3}>T_{1}+\tilde{T}=T_{1}+T_{2}$, contradicting the hypothesis $T_{3}<2 T_{1}$ of Theorem 3.5. This contradiction shows that $m(0)>0$.

As explained before, one of the leaves of the bubbling-off tree obtained from the sequence $\tilde{w}_{n}$ is a cylinder over the orbit $P_{1}$ originated from the puncture $z=0$. By the same arguments used in the proof of theorem 4.20. using the fact that $\mu\left(P_{1}\right)=1$, we conclude that $\mu\left(P_{0}\right) \geq 1$.

Now we show that $\Theta=\emptyset$. Suppose $\Theta \neq \emptyset$. Then by Lemma 4.31, we have $\mu\left(P_{0}\right)=1, \# \Theta=1$ and $\mu(\tilde{P})=2$, where $\tilde{P}$ is the asymptotic limit at the unique puncture in $\Theta$. The orbit $\tilde{P}$ is not linked to $P_{3}$. This is because $\tilde{P}$ is the limit of contractible links contained in the image of the embedded cylinders $w_{n}$. Since $\tilde{T} \leq T_{3}$, by our hypotheses we conclude $\tilde{P}=P_{2}$. But $\tilde{T}$ also satisfies $T_{3}>T_{0}+\tilde{T}>T_{1}+\tilde{T}$, contradicting the assumption $T_{3}<2 T_{1}$. This contradiction proves $\Theta=\emptyset$.

So far, we know that $\tilde{w}: \mathbb{C} \backslash\{0\} \rightarrow \mathbb{R} \times S^{3}$ is a $\tilde{J}$-holomorphic cylinder asymptotic to $P_{3}$ at $\infty$ and to the orbit $P_{0}$ at 0 . By 4.13 , we have $\mu\left(P_{0}\right) \leq 2$, 
so that

$$
1 \leq \mu\left(P_{0}\right) \leq 2 .
$$

The second level of the bubbling-off tree obtained from the sequence $\tilde{w}_{n}$ consists of a unique vertex associated with a finite energy sphere $\tilde{v}_{0}: \mathbb{C} \backslash$ $\{0\} \cup \Theta_{0} \rightarrow \mathbb{R} \times S^{3}$, asymptotic to $P_{0}$ at its positive puncture $\infty$.

Claim: $\mu\left(P_{0}\right)=2$. To prove the claim, suppose by contradiction that $\mu\left(P_{0}\right)=1$. If $\tilde{v}_{0}$ is somewhere injective, using Theorem 2.20, we have

$$
0 \leq 1-\mu\left(P_{0}^{v}\right)-\sum_{z \in \Theta_{0}} \mu\left(P_{z}^{v}\right)+\# \Theta_{0}
$$

where $P_{z}^{v}$ is the asymptotic limit of $\tilde{v}_{0}$ at the puncture $z$. By the same arguments used in the proof of Theorem 4.20, using the fact that $\mu\left(P_{1}\right)=1$, we conclude that $\mu\left(P_{0}^{v}\right) \geq 1$. By Lemma 2.37, we have $\mu\left(P_{z}^{v}\right) \geq 2$. We conclude that $\# \Theta_{0}=0$ and $\pi \cdot d v_{0} \equiv 0$, which contradicts Lemma 4.33.

If $\tilde{v}_{0}$ is not somewhere injective, there exists a somewhere injective $\tilde{J}_{-}$ holomorphic curve $\tilde{u}_{0}: \mathbb{C} \backslash \Gamma \rightarrow \mathbb{R} \times S^{3}$ and a polynomial $p: \mathbb{C} \rightarrow \mathbb{C}$ such that $\tilde{v}_{0}=\tilde{u}_{0} \circ p, p\left(\Theta_{0} \cup\{0\}\right)=\Gamma$ and $p^{-1}(\Gamma)=\Theta_{0} \cup\{0\}$. This implies that $P_{0}=P_{\infty}^{\operatorname{deg} p}$, where $P_{\infty}$ is the asymptotic limit of $\tilde{u}_{0}$ at $\infty$. Using Lemma 1.9 and the assumption $\mu\left(P_{0}\right)=1$, we conclude that $\mu\left(P_{\infty}\right)=1$. For every $z \in \Theta_{0} \cup\{0\}$, we have $P_{z}^{v}=\left(P_{w}^{u}\right)^{k}$, where $p(z)=w, k \mid \operatorname{deg} p$ and $P_{w}^{u}$ is the asymptotic limit of $\tilde{u}_{0}$ at the puncture $w$. Since $\mu\left(P_{z}^{v}\right) \geq 1, \forall z \in \Theta_{0} \cup\{0\}$, using Lemma 1.9 we conclude that $\mu\left(P_{z}^{u}\right) \geq 1, \forall z \in \Gamma$. Applying Theorem 2.20 to the curve $\tilde{u}_{0}$ we have

$$
0 \leq 1-\sum_{z \in \Gamma} \mu\left(P_{z}^{u}\right)+\# \Gamma-1
$$

where $P_{z}^{u}$ is the asymptotic limit of $\tilde{u}_{0}$ at the puncture $z \in \Gamma$. It follows that $\mu\left(P_{z}^{u}\right)=1, \forall z \in \Gamma$. By Theorem 2.20, we have $\pi \cdot d u_{0} \equiv 0$, which implies $\pi \cdot d v_{0} \equiv 0$. It follows from Theorem 2.6 that $\tilde{v}_{0}=F_{P} \circ p$, where $F_{P}$ is a cylinder over an orbit $P \in \mathcal{P}(\lambda), p: \mathbb{C} \rightarrow \mathbb{C}$ is a polynomial and $P_{0}=P^{\operatorname{deg} p}$. Since $\mu\left(P_{0}\right)=1$, we conclude, using Lemma 1.9 , that $\mu(P)=1$. By Lemma 4.33. we have $\Theta_{0} \neq \emptyset$. Let $z \in \Theta_{0}$. Then $\mu\left(P_{z}^{v}\right) \geq 2$ and $P_{z}^{v}=P^{k}$, where $k \mid \operatorname{deg} p$. This implies that $2 \leq \mu\left(P^{k}\right) \leq \mu\left(P^{\operatorname{deg} p}\right)=1$, a contradiction. We have proved the claim $\mu\left(P_{0}\right)=2$.

Now we prove that the mass $m(0)$ of the puncture $z=0$ of $\tilde{v}_{0}$ is zero, according to definition (4.14). This implies that $\tilde{v}_{0}$ is asymptotic to $P_{1}$ at its unique negative puncture $z=0$. 
Claim: $m(0)=0$. Suppose, by contradiction, that $m(0)>0$.

If $\tilde{v}_{0}$ is not somewhere injective, there exists a somewhere injective $\tilde{J}_{-}$ holomorphic curve $\tilde{u}_{0}: \mathbb{C} \backslash \Gamma \rightarrow \mathbb{R} \times S^{3}$ and a polynomial $p: \mathbb{C} \rightarrow \mathbb{C}$ such that $\tilde{v}_{0}=\tilde{u}_{0} \circ p, p\left(\Theta_{0} \cup\{0\}\right)=\Gamma$ and $p^{-1}(\Gamma)=\Theta_{0} \cup\{0\}$. This implies that $P_{0}=P_{\infty}^{\operatorname{deg} p}$, where $P_{\infty}$ is the asymptotic limit of $\tilde{u}_{0}$ at $\infty$. Using Lemma 1.9 . we conclude that $\mu\left(P_{\infty}\right)=1$ and $\operatorname{deg} p=2$. For every $z \in \Theta_{0} \cup\{0\}$, we have $P_{z}^{v}=\left(P_{w}^{u}\right)^{k}$, where $p(z)=w, k \mid \operatorname{deg} p$ and $P_{w}^{u}$ is the asymptotic limit of $\tilde{u}_{0}$ at the puncture $w$. Since $\mu\left(P_{z}^{v}\right) \geq 1, \forall z \in \Theta_{0} \cup\{0\}$, using Lemma 1.9, we conclude that $\mu\left(P_{z}^{u}\right) \geq 1, \forall z \in \Gamma$. Applying Theorem 2.20 to the curve $\tilde{u}_{0}$ we have

$$
0 \leq 1-\sum_{z \in \Gamma} \mu\left(P_{z}^{u}\right)+\# \Gamma-1
$$

where $P_{z}^{u}$ is the asymptotic limit of $\tilde{u}_{0}$ at the puncture $z \in \Gamma$. It follows that $\mu\left(P_{z}^{u}\right)=1, \forall z \in \Gamma$ and $\pi \cdot d u_{0} \equiv 0$. This implies that $\pi \cdot d v_{0} \equiv 0$. It follows from Theorem 2.6 that $\tilde{v}_{0}=F_{P} \circ p$, where $F_{P}$ is a cylinder over an prime orbit $P \in \mathcal{P}(\lambda), p: \mathbb{C} \rightarrow \mathbb{C}$ is a polynomial and $P_{0}=P^{\operatorname{deg} p}$. By Lemma 1.9, we have $\operatorname{deg} p, \mu(P) \in\{1,2\}$. Since, by assumption, $\tilde{v}_{0}$ is not somewhere injective, then $\mu(P)=1$ and $\operatorname{deg} p=2$. By Lemma 4.33. we have $\Theta_{0} \neq \emptyset$. Let $z \in \Theta_{0}$. Then $P_{z}^{v}=P^{k}$, where $k \mid 2=\operatorname{deg} p$. Since we know that $\mu\left(P^{k}\right)=\mu\left(P_{z}^{v}\right) \geq 2$ and $\mu\left(P^{2}\right)=2$, by Lemma 1.9 , we have $\mu\left(P_{z}^{v}\right)=2$. The orbit $\tilde{P}_{z}^{v}$ is not linked to $P_{3}$. This follows from positivity and stability of intersections and the fact that $P_{z}^{v}$ is the limit of contractible links contained in the image of the embedded cylinders $w_{n}$, which do not intersect $P_{3}$. Also, the period of $P_{z}^{v}$ is $<T_{3}$. By the hypotheses of Theorem 3.5, we have $P_{z}^{v}=P_{2}$. This implies $T_{3} \geq T_{0}^{v}+T_{2}>T_{1}+T_{2}$, where $T_{0}^{v}$ is the period of $P_{0}^{v}$, a contradiction with the hypothesis $T_{3}<2 T_{1}$.

If $\tilde{v}_{0}$ is somewhere injective, then we have

$$
0 \leq \operatorname{ind}\left(\tilde{v}_{0}\right)=2-\mu\left(P_{0}^{v}\right)-\sum_{z \in \Theta_{0}} \mu\left(P_{z}^{v}\right)+\# \Theta_{0}
$$

If $\operatorname{ind}\left(\tilde{v}_{0}\right)=0$, then $\pi \cdot d v_{0} \equiv 0$ and by Lemma 4.33 , we have $\Theta_{0} \neq \emptyset$. Using $\mu\left(P_{0}^{v}\right) \geq 1$ and $\mu\left(P_{z}^{v}\right) \geq 2, \forall z \in \Theta_{0}$, we have

$$
1 \leq \mu\left(P_{0}^{v}\right)=2-\sum_{z \in \Theta_{0}} \mu\left(P_{z}^{v}\right)+\# \Theta_{0} \leq 2-\# \Theta_{0}
$$

It follows that $\Theta_{0}=\{z\}$ and $\mu\left(P_{z}^{v}\right)=2$. The orbit $\tilde{P}_{z}^{v}$ is not linked to $P_{3}$ and has period $<T_{3}$. By the hypotheses of Theorem 3.5. we have $P_{z}^{v}=P_{2}$. This implies $T_{3} \geq T_{0}^{v}+T_{2}>T_{1}+T_{2}$, where $T_{0}^{v}$ is the period of $P_{0}^{v}$, a contradiction 
with the hypothesis $T_{3}<2 T_{1}$. If $\operatorname{ind}\left(\tilde{v}_{0}\right) \geq 1$, we have

$$
1 \leq \mu\left(P_{0}^{v}\right) \leq 1-\sum_{z \in \Theta_{0}} \mu\left(P_{z}^{v}\right)+\# \Theta_{0}
$$

Thus $\# \Theta_{0}=\emptyset$ and $\mu\left(P_{0}^{v}\right)=1$. Following the same arguments used to prove the claim $\mu\left(P_{0}\right)=2$, we get a contradiction with $m(0)>0$. We have proved the claim $m(0)=0$. Consequently we have

$$
P_{0}^{v}=P_{1}
$$

We claim that $\Theta_{0}=\emptyset$. To prove this claim, first note that, since $\tilde{v}_{0}$ is asymptotic to $P_{1}$, which is a prime orbit, we know that $\tilde{v}_{0}$ is somewhere injective. Then we have

$$
0 \leq \operatorname{ind}\left(\tilde{v}_{0}\right)=2-\mu\left(P_{1}\right)-\sum_{z \in \Theta_{0}} \mu\left(P_{z}^{v}\right)+\# \Theta_{0}=1-\sum_{z \in \Theta_{0}} \mu\left(P_{z}^{v}\right)+\# \Theta_{0} .
$$

It follows that $\Theta_{0}=\{z\}$ and $\mu\left(P_{z}^{v}\right)=2$. The orbit $\tilde{P}_{z}^{v}$ is not linked to $P_{3}$ and has period $<T_{3}$. By the hypotheses of Theorem 3.5 , we have $P_{z}^{v}=P_{2}$. This implies $T_{3} \geq T_{1}+T_{2}$, which contradicts the hypothesis $T_{3}<2 T_{1}$.

Now we show that $P_{0}=P_{2}$. By positivity and stability of intersections and the fact that the images of the cylinders $\tilde{w}_{n}$ do not intersect $P_{3}$, we conclude that $v_{0}(\mathbb{C} \backslash\{0\})$ does not intersect $P_{3}$. Since $P_{1}$ is not linked to $P_{3}$, we conclude that $P_{0}$ is not linked to $P_{3}$. Since the period of $P_{0}$ is $<T_{3}$ and $P_{0}$ is not linked to $P_{3}$, by the hypotheses of Theorem 3.5, we conclude that

$$
P_{0}=P_{2}
$$

So far, we have proved the following.

Proposition 4.35. Consider a sequence $\tilde{w}_{n}=\left(c_{n}, w_{n}\right): \mathbb{C} \backslash\{0\} \rightarrow \mathbb{R} \times S^{3}$, where $\tilde{w}_{n}=\tilde{w}_{\sigma_{n}}$ and $\sigma_{n} \rightarrow 0^{+}$. There exists a cylinder $\tilde{w}: \mathbb{C} \backslash\{0\} \rightarrow \mathbb{R} \times S^{3}$ asymptotic to $P_{3}$ at its positive puncture $\infty$ and to $P_{2}$ at its negative puncture 0 and a cylinder $\tilde{v}_{0}: \mathbb{C} \backslash\{0\} \rightarrow \mathbb{R} \times S^{3}$ asymptotic to $P_{2}$ at its positive puncture $\infty$ and to $P_{1}$ at its negative puncture 0 , such that, after suitable reparametrizations and $\mathbb{R}$-translations, we have

(i) Up to subsequence, $\tilde{w}_{n} \rightarrow \tilde{w}$ in $C_{l o c}^{\infty}(\mathbb{C} \backslash\{0\})$ as $n \rightarrow \infty$.

(ii) There exist sequences $\delta_{n}^{+} \rightarrow 0^{+}$and $d_{n} \in \mathbb{R}$ such that, up to subsequence, $\tilde{w}_{n}\left(\delta_{n} \cdot\right)+d_{n} \rightarrow \tilde{v}_{0}$ in $C_{\text {loc }}^{\infty}(\mathbb{C} \backslash\{0\})$ as $n \rightarrow \infty$. 
A similar statement holds for any sequence $\tau_{n} \rightarrow 1^{-}$with $\tilde{w}$ replaced with a cylinder $\tilde{w}^{\prime}$ with the same asymptotics as $\tilde{w}$ and $\tilde{v}_{0}$ replaced with a cylinder $\tilde{v}_{0}^{\prime}$ with the same asymptotics as $\tilde{v}_{0}$.

It follows from the uniqueness of the cylinder $\tilde{v}_{r}$ (Proposition 4.24) that $\tilde{v}_{0}=\tilde{v}_{0}^{\prime}=\tilde{v}_{r}$, up to reparametrization and $\mathbb{R}$-translation. By the surjectivity of the gluing map, we conclude that $\tilde{w}=\tilde{u}_{r}$. It follows from the uniqueness of the cylinders $\tilde{u}_{r}$ and $\tilde{u}_{r}^{\prime}$ (Proposition 4.7) that $\tilde{w}^{\prime}$ is either $\tilde{u}_{r}$ or $\tilde{u}_{r}^{\prime}$, up to reparametrization and $\mathbb{R}$-translation.

Proposition 4.36. Consider a sequence $\tilde{w}_{n}=\tilde{w}_{\sigma_{n}}$ in the family $\left\{\tilde{w}_{\tau}\right\}_{\tau \in(0,1)}$ such that $\sigma_{n} \rightarrow 0^{+}$. Then

(i) For any $S^{1}$-invariant neighborhood $\mathcal{W}_{3}$ of the loop $t \mapsto x_{3}\left(T_{3} t\right)$ in $C^{\infty}\left(\mathbb{R} / \mathbb{Z}, S^{3}\right)$, there exists $R_{3}>>1$ such that for $R \geq R_{3}$, the loop $t \mapsto w_{n}\left(R e^{2 \pi i t}\right)$ belongs to $\mathcal{W}_{3}$.

(ii) For any $S^{1}$-invariant neighborhood $\mathcal{W}_{2}$ of the loop $t \mapsto x_{2}\left(T_{2} t\right)$ in $C^{\infty}\left(\mathbb{R} / \mathbb{Z}, S^{3}\right)$, there exist $\epsilon_{2}>0$ and $R_{2}>1$ such that the loop $t \mapsto$ $w_{n}\left(R e^{2 \pi i t}\right)$ belongs to $\mathcal{W}_{2}$ for $R_{2} \delta_{n} \leq R \leq \epsilon_{2}$.

(iii) For any $S^{1}$-invariant neighborhood $\mathcal{W}_{1}$ of the loop $t \mapsto x_{1}\left(T_{1} t\right)$ in $C^{\infty}\left(\mathbb{R} / \mathbb{Z}, S^{3}\right)$, there exist $\epsilon_{1}>0$ such that the loop $t \mapsto w_{n}\left(\rho e^{2 \pi i t}\right)$ belongs to $\mathcal{W}_{1}$ for $\rho \leq \epsilon_{1} \delta_{n}$.

(iv) Given any neighborhood $\mathcal{V} \subset \mathcal{R}_{2}$ of $w(\mathbb{C} \backslash\{0\}) \cup v_{r}(\mathbb{C} \backslash 0) \cup P_{1} \cup P_{2} \cup P_{3}$, we have $w_{n}(\mathbb{C} \backslash\{0\}) \subset \mathcal{V}$, for $n$ large.

A similar statement holds for any sequence $\tilde{w}_{\tau_{n}}$ such that $\tau_{n} \rightarrow 1^{-}$, with $w$ replaced by $w^{\prime}$.

Proof. We can assume that $\mathcal{W}_{i}, i=1,2,3$ contains only the periodic orbit $t \mapsto x_{i}\left(T_{i} \cdot\right)$ modulo $S^{1}$-reparametrizations. Let $\mathcal{W}$ be an $S^{1}$-invariant neighborhood of the set of periodic orbits $P=(x, T) \in \mathcal{P}(\lambda)$ with $T \leq T_{3}$, viewed as maps $x_{T}: S^{1} \rightarrow S^{3}, x_{T}(t)=x(T t)$, such that each of the connected components of $\mathcal{W}$ contains at most one periodic orbit modulo $S^{1}$-reparametrizations and such that $\mathcal{W}_{1} \cup \mathcal{W}_{2} \cup \mathcal{W}_{3} \subset \mathcal{W}$.

Using the normalization condition 4.10) we can apply Lemma 2.29 and find $R_{3}>>1$ such that for $R \geq R_{3}$, the loops $\left\{t \mapsto w_{n}\left(R e^{i 2 \pi t}\right)\right\}, n \in \mathbb{N}$ belong to $\mathcal{W}$. By the asymptotic behavior of the cylinders $\tilde{w}_{n}$, we know that for each $n$, the loop $t \mapsto w_{n}\left(R e^{2 \pi i t}\right)$ belongs to $\mathcal{W}_{3}$ for $R$ large enough. We conclude that for any $R \geq R_{3}$ and $n$ large, the loop $t \mapsto w_{n}\left(R e^{2 \pi i t}\right)$ belongs to $\mathcal{W}_{3}$. Assertion (i) is proved. 
Now we prove (ii). Recall that the asymptotic limit of $\tilde{w}$ at $z=0$ is the orbit $P_{2}$. We can apply 2.29 as in the proof of Proposition 4.32 to find $\epsilon_{2}>0$ small and $R_{2}>>1$ such that for every $R$ satisfying $\delta_{n} R_{2} \leq R \leq \epsilon_{2}$ and $n$ large, the loop $t \mapsto w_{n}\left(R e^{2 \pi i t}\right)$ belongs to $\mathcal{W}_{2}$.

To prove (iii), first recall that the mass of the puncture $z=0$ of the sequence $\tilde{v}_{n}$ is zero. Applying 2.29 as in the proof of Lemma 4.34, we find $\epsilon_{1}>$ 0 small and a sequence $\delta_{n}^{\prime} \rightarrow 0$ such that $t \mapsto w_{n}\left(\delta_{n} \rho^{\prime} e^{2 \pi i t}\right)=v_{n}\left(\rho^{\prime} e^{2 \pi i t}\right) \in \mathcal{W}_{1}$ for $\delta_{n}^{\prime} \leq \rho^{\prime} \leq \epsilon_{1}$. The sequence $v_{n}\left(\delta_{n}^{\prime} \cdot\right)$ converges in $C_{\text {loc }}^{\infty}$ to the cylinder over $P_{1}$, so that applying Lemma 2.29 again, we conclude that for $n$ large, the loop $t \mapsto v_{r}\left(\delta_{n}^{\prime} r e^{2 \pi i t}\right)$ belongs to $\mathcal{W}_{1}$ for every $r \leq 1$. We conclude that for any $\rho \leq \delta_{n} \epsilon_{1}$ and $n$ large enough, the loop $t \mapsto w\left(\rho e^{2 \pi i t}\right)$ belongs to $\mathcal{W}_{1}$.

The proof of (iv) is a consequence of (i), (ii), (iii), the convergence of $\tilde{w}_{n}$ to $\tilde{w}$ in $C_{\text {loc }}^{\infty}(\mathbb{C} \backslash\{0\})$ and the convergence of $\tilde{w}_{n}\left(\delta_{n} \cdot\right)+d_{n}$ to $\tilde{v}_{r}$ in $C_{\text {loc }}^{\infty}(\mathbb{C} \backslash$ $\{0\})$.

The only step left to conclude the proof of Proposition 4.28 is to prove that $\tilde{w}^{\prime}=\tilde{u}_{r}^{\prime}$.

Proposition 4.37. $\tilde{w}^{\prime}=\tilde{u}_{r}^{\prime}$.

Proof. Fix $\tau_{0} \in(0,1)$. The surface

$$
S:=w_{\tau_{0}}(\mathbb{C} \backslash\{0\}) \cup w(\mathbb{C} \backslash\{0\}) \cup v_{r}(\mathbb{C} \backslash\{0\}) \cup P_{1} \cup P_{2} \cup P_{3}
$$

bounds two open connected regions in $S^{3}$. One of these open connected regions, that we call $A$, is contained in $\mathcal{R}_{2}$, because $S$ does not intersect any of the curves foliating the interior of the region $\mathcal{R}_{1}$.

We will show that $A$ is foliated by cylinders in the family $\left\{w_{\tau}\right\}$. Let $p \in A$ and let $\mathcal{V} \subset \mathcal{R}_{2}$ be a small neighborhood of $S$ in $\mathcal{R}_{2}$ separating $p$ and $S$. By Proposition 4.36, for $\tau_{0}^{\prime}<\tau_{0}$ sufficiently small, we have $w_{\tau_{0}^{\prime}}(\mathbb{C} \backslash\{0\}) \subset \mathcal{V}$. Also, $w_{\tau_{0}^{\prime}}(\mathbb{C} \backslash\{0\}) \subset \mathcal{V} \cap A$, since there are points in the image of the family $w_{\tau}$ converging to points in a compact subset of $\tilde{u}_{r}(\mathbb{C} \backslash\{0\})$, as $\tau \rightarrow 0^{+}$. Let $B \subset A$ be the region limited by $w_{\tau_{0}}(\mathbb{C} \backslash\{0\}) \cup P_{3} \cup P_{1} \cup w_{\tau_{0}^{\prime}}(\mathbb{C} \backslash\{0\})$. Thus, the image of the family $w_{\tau}, \tau \in\left[\tau_{0}^{\prime}, \tau_{0}\right]$ is open, closed and non empty in $\bar{B}$. It follows that $p$ is in the image of $w_{\tau}$ for some $\tau \in\left(\tau_{0}^{\prime}, \tau_{0}\right)$. We conclude that $A$ is foliated by the images of the cylinders $\left\{w_{\tau}\right\}, \tau \in\left(0, \tau_{0}\right)$.

Since every neighborhood of a compact set of $u_{r}(\mathbb{C} \backslash\{0\})$ in $\mathcal{R}_{2}$ is contained in $\bar{A}$, the family $w_{\tau}, \tau \in\left(0, \tau_{0}\right)$, foliates $A$ and the cylinders in the family $w_{\tau}$ do not intersect each other, we conclude that $\tilde{w}^{\prime}=\tilde{u}_{r}^{\prime}$.

The proof of Proposition 4.28 is complete. 


\subsubsection{The foliation}

Proposition 4.38. The images of $\left\{w_{\tau}\right\}, \tau \in(0,1), u_{r}, u_{r}^{\prime}, v_{r}, P_{1}, P_{2}$ and $P_{3}$ form a singular foliation of the region $\mathcal{R}_{2}$.

Proof. From the arguments in the proof of Proposition 4.37, we have foliated a region $A$ in $\mathcal{R}_{2}$ bounded by

$$
S:=w_{\tau_{0}}(\mathbb{C} \backslash\{0\}) \cup w(\mathbb{C} \backslash\{0\}) \cup v_{r}(\mathbb{C} \backslash 0) \cup P_{1} \cup P_{2} \cup P_{3},
$$

where $\tau_{0} \in(0,1)$ is fixed. Repeating the same arguments in the proof of Proposition 4.37, with $u_{r}$ replaced by $u_{r}^{\prime}$, we foliate the complement of $A$ in $\mathcal{R}_{2}$.

The proof of Theorem 3.5 is complete. 


\section{Chapter 5}

\section{Idea of the proof of Theorem 3.5 in case $\int_{\mathbb{D} \backslash \Gamma_{0}} u_{0}^{*} d \lambda>0$}

\section{$5.1 \quad$ Gluing}

The gluing process allows us to approximate a broken curve, consisting of two rigid pseudoholomorphic curves sharing a common asymptotic limit, with a family of pseudoholomorphic curves. In this section, we follow the exposition of [Nel13], [Wen16] and [AD14] to state the gluing theorem.

Let $\tilde{J}$ be a regular almost complex structure on the symplectization $\mathbb{R} \times S^{3}$ and let $P_{+}$and $P_{-}$be two closed Reeb orbits. We denote by

$$
\mathcal{S}\left(P_{+}, P_{-}\right)
$$

the moduli space of somewhere injective $\tilde{J}$-holomorphic cylinders converging to $P_{+}$at its positive puncture and to $P_{-}$at its negative puncture, and $\mathcal{S}\left(P_{+}, \emptyset\right)$ the moduli space of $\tilde{J}$-holomorphic planes asymptotic to $P_{+}$.

Remark 5.1. Unless otherwise stated, in what follows the notation $\mathcal{S}\left(P_{+}, P_{-}\right)$ can also denote the case " $P_{-}=\emptyset "$ ", so that our statements can refer both to cylinders and planes.

We define the space of unparametrized trajectories by

$$
\mathcal{M}\left(P_{+}, P_{-}\right):=\mathcal{S}\left(P_{+}, P_{-}\right) /\left(S^{1} \times \mathbb{R}\right)
$$

$\left(\right.$ or $\left.\mathcal{M}\left(P_{+}, \emptyset\right):=\mathcal{S}\left(P_{+}, \emptyset\right) / A u t(\mathbb{C})\right)$ and denote by $\pi: \mathcal{S}\left(P_{+}, P_{-}\right) \rightarrow \mathcal{M}\left(P_{+}, P_{-}\right)$ the quotient map. Consider the $\mathbb{R}$-action on $\mathcal{M}\left(P_{+}, P_{-}\right)$by translations $\tilde{u}=(a, u) \mapsto(a+c, u), c \in \mathbb{R}$. We denote the quotient space by

$$
\hat{\mathcal{M}}\left(P_{+}, P_{-}\right)=\mathcal{M}\left(P_{+}, P_{-}\right) / \mathbb{R} .
$$


Theorem 5.2 (Gluing). Let $P_{x}=\left(x, T_{x}\right), P_{y}=\left(y, T_{y}\right)$ and $P_{z}=\left(z, T_{z}\right)$ (where possibly $P_{z}=\emptyset$ ) be closed Reeb orbits. Let $\tilde{u} \in \mathcal{S}\left(P_{x}, P_{y}\right)$ and $\tilde{v} \in \mathcal{S}\left(P_{y}, P_{z}\right)$ be parametrized solutions with Fredholm index 1, representing equivalence classes $\left(\mathcal{C}_{u}, \mathcal{C}_{v}\right) \in \hat{\mathcal{M}}\left(P_{x}, P_{y}\right) \times \hat{\mathcal{M}}\left(P_{y}, P_{z}\right)$. Then there exists $R_{0} \in \mathbb{R}$ and a differentiable map

$$
\Psi:\left[R_{0}, \infty\right) \rightarrow \mathcal{S}\left(P_{x}, P_{z}\right)
$$

such that $\hat{\Psi}=\pi \circ \Psi$ is an embedding

$$
\hat{\Psi}:\left[R_{0}, \infty\right) \rightarrow \hat{\mathcal{M}}\left(P_{x}, P_{z}\right)
$$

satisfying

$$
\lim _{R \rightarrow+\infty} \hat{\Psi}(R)=\left(\mathcal{C}_{u}, \mathcal{C}_{v}\right)
$$

Theorem 5.3 (Surjectivity of the gluing map). With the same notation of Theorem 5.2, assume that the orbit $P_{y}$ is simply covered. If there exists a sequence $\tilde{w}_{n}$ in $\hat{\mathcal{M}}\left(P_{x}, P_{z}\right)$ converging to $\left(\mathcal{C}_{u}, \mathcal{C}_{v}\right)$, then $\tilde{w}_{n}$ belongs to the image of $\hat{\Psi}$, for $n$ sufficiently large.

\subsubsection{Pregluing}

Given $[\tilde{u}] \in \mathcal{M}\left(P_{+}^{u}, P_{-}^{u}\right)$ and $[\tilde{v}] \in \mathcal{M}\left(P_{+}^{v}, P_{-}^{v}\right)$ such that $P_{-}^{u}=P_{+}^{v}$, we will construct a one parameter family of curves

$$
\bar{w}_{R}=\tilde{u} \#_{R} \tilde{v},
$$

that are not $\tilde{J}$-holomorphic, but are asymptotic to $P_{+}^{u}$ and $P_{-}^{v}$. For each $R$ fixed, the curve $\bar{w}_{R}$ is called preglued map.

Let $P_{-}^{u}=P_{+}^{v}=\left(y, T_{y}\right), P_{+}^{u}=\left(x, T_{x}\right)$ and $P_{-}^{v}=\left(z, T_{z}\right)$. We are assuming that, after fixing $p=y(0)$, cylindrical coordinates $(s, t)$ near $-\infty$ for $\tilde{u}$ and $\left(s^{\prime}, t^{\prime}\right)$ near $+\infty$ for $\tilde{v}$, we have

$$
\lim _{s \rightarrow-\infty} u(s, t)=\lim _{s^{\prime} \rightarrow+\infty} v\left(s^{\prime}, t\right)=y(T t) .
$$

Take a Riemannian metric $g$ on $\mathrm{M}$, that can be defined by $g=\lambda \cdot \lambda+$ $d \lambda(\pi \cdot, J \pi \cdot)$. Writing in coordinates

$$
\tilde{u}(s, t)=(a(s, t), u(s, t)), \quad \tilde{v}\left(s^{\prime}, t^{\prime}\right)=\left(b\left(s^{\prime}, t^{\prime}\right), v\left(s^{\prime}, t^{\prime}\right)\right),
$$


we have

$$
\begin{aligned}
a(s, t) & =T s+\eta(s, t) \\
u(s, t) & =\exp _{y(T t)} U(s, t), \quad \text { for } s<<0 \\
b(s, t) & =T s^{\prime}+\eta^{\prime}\left(s^{\prime}, t^{\prime}\right) \\
v\left(s^{\prime}, t^{\prime}\right) & =\exp _{y(T t)} V\left(s^{\prime}, t^{\prime}\right), \quad \text { for } s>>0
\end{aligned}
$$

where $U$ and $\eta$ decay exponentially as $s \rightarrow-\infty$ and $V, \eta^{\prime}$ decay exponentially as $s^{\prime} \rightarrow+\infty$. This follows from the asymptotic behavior of $\tilde{u}$ and $\tilde{v}$ near the punctures. See Theorem 2.11.

To define the preglued map, we need two cutoff functions $\beta_{R}^{+}$and $\beta_{R}^{-}$in $C^{\infty}(\mathbb{R},[0,1])$ satisfying for $0<\epsilon<\frac{R}{2}$ fixed

$$
\beta_{R}^{-}(s)=\left\{\begin{array}{cc}
1, & s \leq-R \\
0, & s \geq-\epsilon
\end{array} \quad \beta_{R}^{+}(S)=\left\{\begin{array}{cc}
1, & s \geq R \\
0, & s \leq \epsilon
\end{array}\right.\right.
$$

Fix parametrizations of $\tilde{u}$ and $\tilde{v}$ and parametrization of the orbit $(y, T)$ such that (5.1) holds.

Define the preglued map $\bar{w}_{R}(s, t)=\left(c_{R}(s, t), w_{R}(s, t)\right)$ by

$$
\begin{aligned}
w_{R}(s, t) & =\left\{\begin{array}{lr}
v(s+2 R, t), & s \leq-R \\
\exp _{y(T t)}\left(\beta_{R}^{-}(s) V(s+2 R, t)+\beta_{R}^{+}(s) U(s-2 R, t)\right), & s \in[-R, R] \\
u(s-2 R, t), & s \geq R
\end{array}\right. \\
c_{R}(s, t) & =\left\{\begin{array}{lc}
b(s+2 R, t)-2 R T, & s \leq-R \\
T s+\beta_{R}^{-}(s) \eta^{\prime}(s+2 R, t)+\beta_{R}^{+}(s) \eta(s-2 R, t), & s \in[-R, R] \\
a(s-2 R, t)+2 R T, & s \geq R
\end{array}\right.
\end{aligned}
$$

The map $\bar{w}_{R}$ is defined for $R$ sufficiently large, such that for $s \in[-R, R]$, $u(s-2 R, t)=\exp _{y(T t)} U(s-2 R, t)$ and $v(s+2 R, t)=\exp _{y(T t)} V(s+2 R, t)$.

If $\tilde{v}$ is a plane, we have not defined $\bar{w}_{R}(0)$ yet. Recall that we write $\tilde{v}(s, t)=v\left(e^{2 \pi(s+i t)}\right)$. We define for $z \in \overline{B_{e^{-2 \pi R}(0)}}$,

$$
\bar{w}_{R}(z)=\tau_{-2 R T} \circ \tilde{v}\left(e^{4 \pi R} z\right),
$$

where the map $\tau_{c}: \mathbb{R} \times S^{3} \rightarrow \mathbb{R} \times S^{3}$ is defined by $\tau_{c}(a, x)=(a+c, x)$, for $c \in \mathbb{R}$.

\section{Properties of the preglued map}

- $\bar{w}_{R}$ is asymptotic to $P_{+}^{u}$ at $z=\infty$ and asymptotic to $P_{-}^{v}$ at $z=0$.

- for $s \in[-\epsilon, \epsilon], \bar{w}_{R}(s, t)=(T s, y(T t))$. 
- If $s \leq R, \bar{w}_{R}(s-2 R, t)=(b(s, t)-2 R T, v(s, t))$. It follows that $\tau_{2 R T} \circ$ $\bar{w}_{R}(s-2 R, t) \rightarrow \tilde{v}(s, t)$ in $C_{l o c}^{\infty}$ as $R \rightarrow \infty$.

- If $s \geq-R, \bar{w}_{R}(s+2 R, t)=(a(s, t)+2 R T, u(s, t))$. It follows that $\tau_{-2 R T} \circ \bar{w}_{R}(s+2 R, t) \rightarrow \tilde{u}(s, t)$ in $C_{l o c}^{\infty}$ as $R \rightarrow \infty$.

- $\bar{w}_{R}$ converges to $(T s, y(T t))$ as $R \rightarrow \infty$ in $C_{l o c}^{\infty}$.

- $\bar{\partial} \bar{w}_{R} \rightarrow 0$ in $C_{l o c}^{\infty}$.

Construction of the gluing map Using a version of the implicit function theorem, one can show that there exists a distinguished $\tilde{J}$-holomorphic curve $\tilde{w}_{R}=\Psi(R) \in \mathcal{S}\left(P_{+}^{u}, P_{-}^{v}\right)$ close to the preglued map $\bar{w}_{R}$ for $R$ sufficiently large, which has the form $\Psi(R)=\exp _{\bar{w}_{R}}(\eta(R))$, where $\eta(R) \in W_{\delta}^{1, p}\left(\bar{w}_{R}^{*} T W\right)$. This can be proved by the same arguments found in [AD14 and the Fredholm theory of [Dra04].

\subsection{Idea of the proof of Theorem 3.5 in the case $\int_{\mathbb{D} \backslash \Gamma_{0}} u_{0}^{*} d \lambda>0$}

In Section 3.3.4, we obtained a $\tilde{J}$-holomorphic curve $\tilde{u}_{0}: \mathbb{D} \backslash\{0\} \rightarrow \mathbb{R} \times S^{3}$ satisfying

$$
\left\{\begin{array}{l}
\tilde{u}_{0}=\left(a_{0}, u_{0}\right): \mathbb{D} \backslash\{0\} \rightarrow \mathbb{R} \times S^{3} \text { is an embedding } \\
a_{0} \equiv 0 \text { on } \partial \mathbb{D}, u_{0}(\partial \mathbb{D}) \subset \mathcal{D} \backslash\{e\} \\
u_{0}(\partial \mathbb{D}) \text { winds once positively around } e \\
\tilde{u}_{0} \text { is asymptotic to } P_{2} \text { at } z=0
\end{array}\right.
$$

where $\mathcal{D}$ is the disk spanning the orbit $P_{3}$ obtained by Theorem 3.10 . We also obtained a $\tilde{J}$-holomorphic plane $\tilde{v}: \mathbb{C} \rightarrow \mathbb{R} \times S^{3}$ asymptotic to $P_{2}$.

By Proposition 4.20 , we obtain a $\tilde{J}$-holomorphic cylinder $\tilde{v}_{r}: \mathbb{C} \backslash\{0\} \rightarrow$ $\mathbb{R} \times S^{3}$ asymptotic to $P_{2}$ at its positive puncture $z=\infty$ and to $P_{1}$ at its negative puncture $z=0$. By Proposition 4.24, we know that $\tilde{v}$ and $\tilde{v}_{r}$ approach the orbit $P_{2}$ in opposite directions, according to definition 4.6 .

Now we can apply the Gluing theorem 5.2 to the curves $\tilde{u}_{0}$ and $\tilde{v}_{r}$ to obtain a 1-parameter family of $\tilde{J}$-holomorphic punctured disks

$$
\tilde{u}_{R}=\left(a_{R}, u_{R}\right): \mathbb{D} \backslash\{0\} \rightarrow \mathbb{R} \times S^{3}, R \in\left[R_{0},+\infty\right)
$$


satisfying

$$
\left\{\begin{array}{l}
a_{R} \equiv 0 \text { on } \partial \mathbb{D}, u_{R}(\partial \mathbb{D}) \subset \mathcal{D} \backslash\{e\} \\
u_{R}(\partial \mathbb{D}) \text { winds once and positively around } e \\
\tilde{u}_{R}(\mathbb{D} \backslash\{0\}) \cap\left(\mathbb{R} \times x_{3}(\mathbb{R})\right)=\emptyset \\
\tilde{u}_{R} \text { is asymptotic to } P_{1} \text { at } z=0
\end{array}\right.
$$

We need the following proposition, whose proof is not given in this thesis.

Proposition 5.4. Let $\tilde{u}=(a, u): \mathbb{D} \backslash\{0\} \rightarrow \mathbb{R} \times S^{3}$ be a $\tilde{J}$-holomorphic curve satisfying

$$
\left\{\begin{array}{l}
a \equiv 0 \text { on } \partial \mathbb{D} \\
u(\partial \mathbb{D}) \subset \mathcal{D} \backslash\{e\}, \\
\tilde{u} \text { is asymptotic to } P_{2} \text { at } z=0
\end{array}\right.
$$

Consider two $\tilde{J}$-holomorphic curves $\tilde{v}: \mathbb{C} \backslash \Gamma_{v} \rightarrow \mathbb{R} \times S^{3}$ and $\tilde{v}^{\prime}: \mathbb{C} \backslash$ $\Gamma_{v^{\prime}} \rightarrow \mathbb{R} \times S^{3}$, asymptotic to $P_{2}$ at its unique positive puncture $z=\infty$, with $\# \Gamma_{v}, \# \Gamma_{v^{\prime}} \leq 1$ and such that $\tilde{v}$ and $\tilde{v}^{\prime}$ approach $P_{2}$ in opposite directions. Let $\tilde{w}_{R}: \mathbb{D} \backslash \Gamma_{v} \rightarrow \mathbb{R} \times S^{3}, R \geq R_{0}$ be the family of $\tilde{J}$-holomorphic curves given by Theorem 5.2 applied to the curves $\tilde{u}$ and $\tilde{v}$, and let $\tilde{w}_{R}^{\prime}: \mathbb{D} \backslash \Gamma_{v} \rightarrow \mathbb{R} \times S^{3}$, $R \geq R_{0}^{\prime}$ be the family of $\tilde{J}$-holomorphic curves given by Theorem 5.2 applied to the curves $\tilde{u}$ and $\tilde{v}^{\prime}$. Then for $R$ and $s$ sufficiently large, the following holds: If $z=e^{2 \pi(s+i t)}$ and $\tilde{w}_{R}(z)=\exp _{\tilde{u} \#_{R} \tilde{v}} V(z)$, where $V$ is a section on $\tilde{u} \#_{R} \tilde{v}^{*} T\left(\mathbb{R} \times S^{3}\right)$, then $\tilde{w}_{R}^{\prime}(z)=\exp _{\tilde{u} \#_{R} \tilde{v}^{\prime}}(-V(z))$.

By surjectivity of gluing (Theorem 5.3), we know that the family of disks given by Theorem 5.2 applied to the curves $\tilde{u}_{0}$ and $\tilde{v}$ coincide with the family of disks obtained in Section 3.3.2.

Recall that in Section 3.3.2, we fixed a leaf $l_{1}$ of the characteristic foliation of $\mathcal{D}$ and denoted its length by $\bar{\tau}$. We defined for each disk $\tilde{u} \in \mathcal{M}(J)$,

$$
\tau([u])=\text { length of the piece of } l_{1} \text { connecting } e \text { to } p_{*}(\tilde{u})
$$

where $p_{*}(\tilde{u})$ is the intersection point of $l_{1}$ and $u(\partial \mathbb{D}) . \tau(u)$ is well defined because $u(\partial \mathbb{D})$ hits every leaf exactly once. In the same way we can define define $\tau^{*}=\tau\left(\tilde{u}_{0}\right)=$ length of the piece of $l_{1}$ connecting $e$ to $p_{*}\left(\tilde{u}_{0}\right)$, where $p_{*}\left(\tilde{u}_{0}\right)$ is the intersection point of $l_{1}$ and $u_{0}(\partial \mathbb{D})$.

Now we apply Proposition 5.4 to the curves $\tilde{u}_{0}, \tilde{v}$ and $\tilde{v}_{r}$. One can show that for $R$ large, any curve $\tilde{u}_{R}$ in the family (5.4) satisfies $\tau\left(\left[\tilde{u}_{R}\right]\right)>\tau^{*}$, where we also define $\tau\left(\left[\tilde{u}_{R}\right]\right)=$ length of the piece of $l_{1}$ connecting $e$ to $p_{*}\left(\tilde{u}_{R}\right)$ and $p_{*}\left(\tilde{u}_{R}\right)$ is the intersection point of $l_{1}$ and $u_{R}(\partial \mathbb{D})$. 
The family of cylinders $\tilde{u}_{R}, R \in\left[R_{0},+\infty\right)$ extends to a maximal family

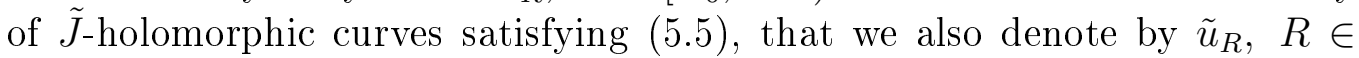
$\left(R_{-},+\infty\right)$.

We need the following facts about the family $\tilde{u}_{R}$, that are left without proof.

1. $\tilde{u}_{R}$ is an embedding near $\partial \mathbb{D}$;

2. $\tilde{u}_{R}$ is an immersion for all $R \in\left(R_{-},+\infty\right)$;

3. for $R \neq R^{\prime}, \tilde{u}_{R}(\partial \mathbb{D}) \cap \tilde{u}_{R^{\prime}}(\partial \mathbb{D})=\emptyset$.

Consider a sequence $\tilde{u}_{n}=\tilde{u}_{R_{n}}$, such that $R_{n} \rightarrow R_{-}^{+}$. After an analysis similar to the one done in Sections 4.4.1 and 4.4.2, we can show that, after suitable reparametrizations and $\mathbb{R}$-translations, we have the following

(i) There exists a $\tilde{J}$-holomorphic curve $\tilde{u}_{1}: \mathbb{D} \backslash\{0\} \rightarrow \mathbb{R} \times S^{3}$ such that, up to subsequence, $\tilde{u}_{n} \rightarrow \tilde{u}_{1}$ in $C_{l o c}^{\infty}(\mathbb{D} \backslash\{0\})$ as $n \rightarrow \infty$.

(ii) There exist sequences $\delta_{n} \rightarrow 0^{+}, R_{n} \rightarrow+\infty$ and $d_{n} \in \mathbb{R}$, and a $\tilde{J}_{-}$ holomorphic curve $\tilde{v}_{1}: \mathbb{C} \backslash\{0\} \rightarrow \mathbb{R} \times S^{3}$ such that, up to subsequence, defining $\tilde{w}_{n}: B_{R_{n}}(0) \backslash\{0\} \rightarrow \mathbb{R} \times S^{3}$ by $\tilde{w}_{n}=\tilde{u}_{n}\left(\delta_{n} \cdot\right)+d_{n}$, we have $\tilde{w}_{n} \rightarrow \tilde{v}_{1}$ in $C_{l o c}^{\infty}(\mathbb{C} \backslash\{0\})$ as $n \rightarrow \infty$.

Note that, since each curve $\tilde{u}_{R}$ satisfies $\tau\left(\left[\tilde{u}_{R}\right]\right)>\tau^{*}$ and $\tau\left(\left[\tilde{u}_{R}\right]\right)$ is a decreasing function of $R$, we have $\tau\left(\left[\tilde{u}_{1}\right]\right)>\tau^{*}$.

If $\pi \circ d u_{1} \equiv 0$, then, by the same arguments of Proposition 3.18, we know that $\tilde{u}_{1}$ is a reparametrization of the $\tilde{J}$-holomorphic curve $F: \mathbb{D} \backslash\{0\} \rightarrow$ $\mathbb{R} \times S^{3}, F\left(z=e^{2 \pi(s+i t)}\right)=\left(T_{3} s, x_{3}\left(T_{3} t\right)\right)$, and $\tilde{v}_{1}$ is a cylinder asymptotic to $P_{3}$ at its unique positive puncture $z=\infty$ and asymptotic to either $P_{2}$ or $P_{1}$ at its unique negative puncture $z=0$.

If $\int_{\mathbb{D} \backslash\{0\}} u_{1}^{*} d \lambda>0$, then $\tilde{u}_{1}$ is asymptotic to $P_{2}$ at its unique negative puncture $z=0$ and we can apply the Gluing theorem 5.2 to the curves $\tilde{u}_{1}$ and $\tilde{v}$ to obtain a family of disks

$$
\tilde{u}_{R}^{1}: \mathbb{D} \rightarrow \mathbb{R} \times S^{3}, R \in\left[R_{1},+\infty\right),
$$

such that for each $R, \tau\left(\left[\tilde{u}_{R}^{1}\right]\right)>\tau\left(\left[\tilde{u}_{1}\right]\right)>\tau^{*}$.

We claim that there exists a family of $\tilde{J}$-holomorphic disks $\tilde{u}_{n}=\left(a_{n}, u_{n}\right)$ : $\mathbb{D} \rightarrow \mathbb{R} \times S^{3}$ satisfying

$$
\left\{\begin{array}{l}
a_{n} \equiv 0 \text { on } \partial \mathbb{D}, \quad u_{n}(\partial \mathbb{D}) \subset \mathcal{D} \backslash\{e\} \\
u_{n}(\partial \mathbb{D}) \text { winds once positively around } e \\
\tilde{u}_{n}(\mathbb{D} \backslash\{0\}) \cap\left(\mathbb{R} \times x_{3}(\mathbb{R})\right)=\emptyset
\end{array}\right.
$$


Such that $\tau\left(\left[\tilde{u}_{n}\right]\right) \rightarrow \bar{\tau}$ as $n \rightarrow \infty$, or a family of $\tilde{J}$-holomorphic punctured disks $\tilde{w}_{n}=\left(c_{n}, w_{n}\right): \mathbb{D} \backslash\{0\} \rightarrow \mathbb{R} \times S^{3}$ satisfying

$$
\left\{\begin{array}{l}
c_{n} \equiv 0 \text { on } \partial \mathbb{D}, w_{n}(\partial \mathbb{D}) \subset \mathcal{D} \backslash\{e\}, \\
w_{n}(\partial \mathbb{D}) \text { winds once and positively around } e, \\
\tilde{w}_{n} \text { is asymptotic to } P_{1} \text { at } z=0 .
\end{array}\right.
$$

such that $\tau\left(\left[\tilde{w}_{n}\right]\right) \rightarrow \bar{\tau}$ as $n \rightarrow \infty$. The argument to proof the claim is as follows.

Suppose, by contradiction, that no such families of $\tilde{J}$-holomorphic curves exist. Let $\tau_{\infty}$ be the supremum of $\tau([\tilde{u}])$ over all $\tilde{J}$-holomorphic disks $\tilde{u}$ : $\mathbb{D} \rightarrow \mathbb{R} \times S^{3}$ satisfying (5.7) and let $\tilde{u}_{n}$ be a sequence of disks satisfying (5.7) and such that $\tau\left(\left[\tilde{u}_{n}\right]\right) \rightarrow \tau_{\infty}$ as $n \rightarrow \infty$. Repeating the analysis done in Section 3.3 .2 , we find a $\widetilde{J}$-holomorphic punctured disk

$$
\tilde{u}_{\infty}: \mathbb{D} \backslash\{0\} \rightarrow \mathbb{R} \times S^{3}
$$

asymptotic to $P_{2}$ at $z=0$ such that $\tilde{u}_{n} \rightarrow \tilde{u}_{\infty}$ in $C_{l o c}^{\infty}(\mathbb{D} \backslash\{0\})$ as $n \rightarrow \infty$ and $\tau\left(\tilde{u}_{\infty}\right)=\tau_{\infty}$. Also, there exist sequences $\delta_{n} \rightarrow 0^{+}$and $d_{n} \in \mathbb{R}$ such that, up to subsequence, we have $\tilde{u}_{n}\left(\delta_{n} \cdot\right)+d_{n} \rightarrow \tilde{v}$ in $C_{\text {loc }}^{\infty}(\mathbb{C})$ as $n \rightarrow \infty$. Applying the Gluing theorem 5.2 to the punctured disk $\tilde{u}_{\infty}$ and the cylinder $\tilde{v}_{r}$, we obtain a family of $\tilde{J}$-holomorphic punctured disks

$$
\tilde{u}_{R}^{\infty}=\left(a_{R}^{\infty}, u_{R}^{\infty}\right): \mathbb{D} \backslash\{0\} \rightarrow \mathbb{R} \times S^{3}, R \in\left[R^{\infty},+\infty\right)
$$

satisfying

$$
\left\{\begin{array}{l}
a_{R}^{\infty} \equiv 0 \text { on } \partial \mathbb{D}, u_{R}^{\infty}(\partial \mathbb{D}) \subset \mathcal{D} \backslash\{e\}, \\
u_{R}^{\infty}(\partial \mathbb{D}) \text { winds once and positively around } e, \\
\tilde{u}_{R}(\mathbb{D} \backslash\{0\}) \cap\left(\mathbb{R} \times x_{3}(\mathbb{R})\right)=\emptyset, \\
u_{R}^{\infty} \text { is asymptotic to } P_{1} \text { at } z=0 .
\end{array}\right.
$$

Applying Proposition 5.4 to the curves $\tilde{u}_{\infty}, \tilde{v}$ and $\tilde{v}_{r}$, one can show that for $R$ large, we have $\bar{\tau}>c>\tau\left(\left[\tilde{u}_{R}^{\infty}\right]\right)>\tau_{\infty}$.

The family $\tilde{u}_{R}^{\infty}$ extends to a maximal family of $\tilde{J}_{\text {-holomorphic punctured }}$ disks satisfying (5.9), that we also denote by $\tilde{u}_{R}^{\infty}, R \in\left(R_{-},+\infty\right)$. Taking the limit as $R \rightarrow R_{-}$, we obtain a $\tilde{J}$-holomorphic punctured disk $\tilde{w}_{\infty}: \mathbb{D} \backslash\{0\} \rightarrow$ $\mathbb{R} \times S^{3}$ asymptotic to $P_{2}$ at its unique negative puncture and satisfying

$$
\bar{\tau}>\tau\left(\left[\tilde{w}_{\infty}\right]\right)>\tau_{\infty}
$$


Gluing $\tilde{w}_{\infty}$ with the plane $\tilde{v}$ and applying Proposition 5.4, one can show that there exists a family of disks $\tilde{w}_{R}: \mathbb{D} \rightarrow \mathbb{R} \times S^{3}$ satisfying (5.7) and such that $\tau\left(\left[\tilde{w}_{R}\right]\right)>\tau_{\infty}$, a contradiction.

We conclude that there exists either

1. a family of $\tilde{J}$-holomorphic disks $\tilde{u}_{n}=\left(a_{n}, u_{n}\right): \mathbb{D} \rightarrow \mathbb{R} \times S^{3}$ satisfying (5.7) and such that $\tau\left(\left[\tilde{u}_{n}\right]\right) \rightarrow \bar{\tau}$ as $n \rightarrow \infty$. Following the same arguments used in Section 3.3.2, after suitable reparametrizations and $\mathbb{R}$-translations, we have the following:

(i) Passing to subsequence, $\tilde{u}_{n} \rightarrow F$ in $C_{l o c}^{\infty}(\mathbb{D} \backslash\{0\})$ as $n \rightarrow \infty$, where $F\left(z=e^{2 \pi(s+i t)}\right)=\left(T_{3} s, x_{3}\left(T_{3} t\right)\right)$;

(ii) There exist sequences $\delta_{n} \rightarrow 0^{+}, R_{n} \rightarrow+\infty$ and $d_{n} \in \mathbb{R}$, and a $\tilde{J}$-holomorphic curve $\tilde{v}_{\infty}: \mathbb{C} \backslash \Gamma_{\infty} \rightarrow \mathbb{R} \times S^{3}$ such that, up to subsequence, defining $\tilde{w}_{n}: B_{R_{n}}(0) \rightarrow \mathbb{R} \times S^{3}$ by $\tilde{w}_{n}=\tilde{u}_{n}\left(\delta_{n} \cdot\right)+d_{n}$, we have $\tilde{w}_{n} \rightarrow \tilde{v}_{\infty}$ in $C_{\text {loc }}^{\infty}\left(\mathbb{C} \backslash \Gamma_{\infty}\right)$ as $n \rightarrow \infty$. The curve $\tilde{v}_{\infty}$ is either a plane asymptotic to $P_{3}$ or $\Gamma_{\infty}=\{0\}$ and $\tilde{v}_{\infty}$ is a cylinder asymptotic to $P_{3}$ at its positive puncture $\infty$ and to $P_{2}$ at its negative puncture $z=0$;

(iii) If $\Gamma_{\infty}=\{0\}$, there exist sequences $\delta_{n}^{\prime} \rightarrow 0^{+}$and $d_{n}^{\prime} \in \mathbb{R}$ such that, up to subsequence, we have $\tilde{u}_{n}\left(\delta_{n}^{\prime} \cdot\right) \rightarrow \tilde{v}$ in $C_{l o c}^{\infty}(\mathbb{C})$ as $n \rightarrow \infty$.

2. or a family of $\tilde{J}$-holomorphic punctured disks $\tilde{u}_{n}=\left(a_{n}, u_{n}\right): \mathbb{D} \backslash\{0\} \rightarrow$ $\mathbb{R} \times S^{3}$ satisfying (5.8) and such that $\tau\left(\left[\tilde{u}_{n}\right]\right) \rightarrow \bar{\tau}$ as $n \rightarrow \infty$. Following the same arguments used in Sections 3.3 .2 and 4.4.1, after suitable reparametrizations and $\mathbb{R}$-translations, we have the following

(i) Passing to subsequence, $\tilde{u}_{n} \rightarrow F$ in $C_{l o c}^{\infty}(\mathbb{D} \backslash\{0\})$ as $n \rightarrow \infty$, where $F\left(z=e^{2 \pi(s+i t)}\right)=\left(T_{3} s, x_{3}\left(T_{3} t\right)\right)$;

(ii) There exist sequences $\delta_{n} \rightarrow 0^{+}, R_{n} \rightarrow+\infty$ and $d_{n} \in \mathbb{R}$, and a $\tilde{J}$-holomorphic curve $\tilde{v}_{\infty}: \mathbb{C} \backslash\{0\} \rightarrow \mathbb{R} \times S^{3}$ such that, up to subsequence, we have $\tilde{u}_{n}\left(\delta_{n} \cdot\right) \rightarrow \tilde{v}_{\infty}$ in $C_{\text {loc }}^{\infty}\left(\mathbb{C} \backslash \Gamma_{\infty}\right)$ as $n \rightarrow \infty$. The curve $\tilde{v}_{\infty}$ is either a cylinder asymptotic to $P_{3}$ at its positive puncture $z=\infty$ and to $P_{1}$ at its negative puncture $z=0$ or a cylinder asymptotic to $P_{3}$ at its positive puncture $\infty$ and to $P_{2}$ at its negative puncture $z=0$;

(iii) If $\tilde{v}$ is asymptotic to $P_{2}$ at $z=0$, there exist sequences $\delta_{n}^{\prime} \rightarrow 0^{+}$ and $d_{n}^{\prime} \in \mathbb{R}$ such that, up to subsequence, we have $\tilde{u}_{n}\left(\delta_{n}^{\prime} \cdot\right) \rightarrow \tilde{v}_{r}$ in $C_{\text {loc }}^{\infty}(\mathbb{C} \backslash\{0\})$ as $n \rightarrow \infty$.

In both cases, we can prove Theorem 3.5 by the same arguments of Chapter 4 . 


\section{Appendix A}

\section{Proof of Lemma 3.9}

In this Appendix, we prove Lemma 3.9, which is restated below.

Lemma A.1. Assume that $\psi: S^{2} \rightarrow S^{3}$ is a $C^{1}$ embedding such that $x_{2}\left(T_{2} \cdot\right)=\left.\psi\right|_{S^{1} \times\{0\}}$ and such that each hemisphere is a strong transverse section. Then $\psi$ is transverse to the torus $T$ along $P_{2}$.

Let $P=(x, T) \in \mathcal{P}(\lambda)$ be a prime orbit such that $\mu(P)=2$. The orbit $P$ is hyperbolic and lies in the intersection of its stable manifold $W^{+}(P)$ and its unstable manifold $W^{-}(P)$. The tangent spaces of $W^{ \pm}(P)$ along the periodic solution $t \mapsto x(t)$ are spanned by the Reeb vector field $R(x(t))$ and vector fields $v^{ \pm}(t) \in \xi_{x(t)}$.

Fix a symplectic trivialization $\Psi: x_{T}^{*} \xi \rightarrow \mathbb{R} / \mathbb{Z} \times \mathbb{R}^{2}$. Let

$$
\Phi(t)=\left.\Psi_{t / T} \circ d \varphi^{t}\right|_{\xi(x(0))} \circ \Psi_{0}^{-1} .
$$

We know that $\Phi(T)$ has two eigenvalues $\beta, \beta^{-1}$, where $\beta>1$.

For each $t, v^{-}(t)$ is an eigenvector of $d \varphi^{T} \mid \xi(x(t))$ associated with the eigenvalue $\beta$. In the same way, $v^{+}(t)$ is an eigenvector of $d \varphi^{T} \mid \xi(x(t))$ associated with the eigenvalue $\beta^{-1}$. Changing $v^{ \pm}(t)$ with $-v^{ \pm}(t)$ if necessary, we can assume $\left\{v^{-}(t), v^{+}(t)\right\}$ is a positive basis for $\xi_{x(t)}$ for each $t$. The basis $\left\{v^{-}(t), v^{+}(t)\right\}$ determines four open quadrants in $\xi_{x(t)}$. Let (I) and (III) be the open quadrants between $\mathbb{R} v^{-}(t)$ and $\mathbb{R} v^{+}(t)$ following the positive orientation and (II) and (IV) the open quadrants between $\mathbb{R} v^{+}(t)$ and $\mathbb{R} v^{-}(t)$.

Proposition A.2. Let $t \mapsto v(t) \in \xi_{x(T t)}$ be a section such that

$$
\operatorname{wind}(v, \Psi)=1 \text {. }
$$

If $d \lambda\left(v(t), \mathcal{L}_{R} v(t)\right)>0, \forall t \in S^{1}$, then $v(t)$ belongs to regions $(I)$ or $(I I I)$ for every $t \in S^{1}$. If $d \lambda\left(v(t), \mathcal{L}_{R} v(t)\right)<0, \forall t \in S^{1}$, then $v(t)$ belongs to regions $(I I)$ or $(I V)$ for every $t \in S^{1}$. 
Proof. Let $S(t)=-J_{0} \dot{\Phi}(t) \Phi^{-1}(t)$. Recall that $t \mapsto S(t)$ is $T$-periodic, and for each $t, S(t)$ is real and symmetric. Define $A(t)=J_{0} S(t)$.

The sections $v^{ \pm}(t)$ are solutions of

$$
\dot{x}(t)=A(t) x(t)
$$

satisfying

$$
v^{+}(T)=\frac{1}{\beta} v^{+}(0), \quad v^{-}(T)=\beta v^{-}(0)
$$

We will use Floquet theory to change coordinates so that the equation A.1 changes to

$$
\dot{y}(t)=B y(t)
$$

with $B$ constant.

In the basis $\left\{v^{-}(0), v^{+}(0)\right\}, \Phi(T)$ has the form

$$
\Phi(T)=\left[\begin{array}{cc}
\beta & 0 \\
0 & \frac{1}{\beta}
\end{array}\right]
$$

We want to find a matrix $B$ satisfying $e^{T B}=\Phi(T)$ and a $T$-periodic map $t \mapsto P(t)$ such that $\Phi(t)=P(t) e^{t B}, \forall t \in \mathbb{R}$, so that with the change of variables $y=P^{-1}(t) x$, the equation A.1 becomes $\dot{y}(t)=B y(t)$.

Define

$$
B=\frac{1}{T}\left[\begin{array}{cc}
\ln \beta & 0 \\
0 & -\ln \beta
\end{array}\right]
$$

and $t \mapsto P(t)$ by

$$
\Phi(t)=P(t) e^{t B}
$$

The map $t \mapsto P(t)$ is $T$-periodic. In fact,

$$
\Phi(t) e^{T B}=\Phi(t) \Phi(T)=\Phi(t+T)=P(t+T) e^{t B} e^{T B} \Rightarrow \Phi(t)=P(t+T) e^{t B} .
$$

If $x(t)$ is a solution of $\dot{x}=A(t) x(t)$, then $y(t)=P(t)^{-1} x(t)$ satisfy

$$
\begin{aligned}
\dot{y}(t) & =\left(P(\dot{t})^{-1}\right) x(t)+P(t)^{-1} \dot{x}(t) \\
& =-P(t)^{-1} \dot{P}(t)+P(t)^{-1}(P(t) y(t))+P(t)^{-1} A(t)(P(t) y(t)) \\
& =\left(-P(t)^{-1} \dot{P}(t)+P(t)^{-1} A(t) P(t)\right) y(t) .
\end{aligned}
$$


Using the identities $\dot{\Phi}(t)=A(t) \Phi(t)$ and $\Phi(t)=P(t) e^{t B}$, we get

$$
\begin{aligned}
& A(t) P(t) e^{t B}=\dot{\Phi}(t)=P(t) e^{t B} B+\dot{P}(t) e^{t B} \\
& \Rightarrow P(t) B+\dot{P}(t)=A(t) P(t) \\
& \Rightarrow B=-P(t)^{-1} \dot{P}(t)+P(t)^{-1} A(t) P(t) .
\end{aligned}
$$

Then $\dot{y}(t)=B y(t)$. In the same way, if $y(t)$ solves $\dot{y}(t)=B y(t)$, then $x(t)=P(t) y(t)$ solves (A.1).

In the basis $\left\{P^{-1}(t) v^{-}(0), P^{-1}(t) v^{+}(0)\right\}$, the equation $\dot{y}(t)=B y(t)$ becomes

$$
\left\{\begin{array}{l}
\dot{y_{1}}(t)=\lambda y_{1}(t) \\
\dot{y_{2}}(t)=-\lambda y_{2}(t)
\end{array}\right.
$$

where $\lambda=\frac{1}{T} \ln \beta$.

Writing

$$
\left\{\begin{array}{l}
y_{1}(t)=\rho(t) \cos \eta(t) \\
y_{2}(t)=\rho(t) \sin \eta(t)
\end{array}\right.
$$

We get

$$
\rho^{2}(t) \dot{\zeta}(t)=\rho^{2}(t)(-2 \lambda \sin \zeta(t) \cos \zeta(t)) .
$$

Let $t \mapsto v(t) \in \xi_{x(t)}$ be a section on $x^{*} \xi$. We want to compute

$$
d \lambda\left(v(t), \mathcal{L}_{R} v(t)\right)
$$

In the symplectic trivialization $\Psi, \mathcal{L}_{R} v(t)$ takes the form

$$
\mathcal{L}_{R} v(t)=\frac{d}{d t} v(t)-J_{0} S(t) v(t)
$$

So that

$$
\begin{aligned}
d \lambda\left(v(t), \mathcal{L}_{R} v(t)\right) & =d \lambda_{0}\left(v(t), \dot{v}(t)=J_{0} S(t) v(t)\right) \\
& =d \lambda_{0}(v(t), \dot{v}(t))-d \lambda_{0}(v(t), A(t) v(t)) .
\end{aligned}
$$

Writing $v(t)$ in the basis $\left\{v^{-}(0), v^{+}(0)\right\}$ as

$$
v(t)=(r(t) \cos \theta(t), r(t) \sin \theta(t)),
$$

we have

$$
d \lambda_{0}(v(t), \dot{v}(t))=C r(t)^{2} \dot{\theta}(t),
$$

where $C$ is a positive constant.

For $t$ fixed, let $s \mapsto x_{t}(s)$ be the solution of A.1 such that $x_{t}(t)=v(t)$. 
Writing $x_{t}(s)=x_{1}(s) v^{-}(0)+x_{2}(s) v^{+}(0)$, we have

$$
P^{-1}(t) v(t)=x_{1}(t)\left(P(t)^{-1} v^{-}(0)\right)+x_{2}\left(P(t)^{-1} v^{+}(0)\right)
$$

so that the functions $x_{1}$ and $x_{2}$ satisfy

$$
\left\{\begin{array}{l}
\dot{x_{1}}(t)=\lambda x_{1}(t) \\
\dot{x_{2}}(t)=-\lambda x_{2}(t)
\end{array}\right.
$$

Then

$$
\begin{aligned}
A(t) v(t) & =A(t) x_{t}(t)=\dot{x}_{t}(t) \\
& =\dot{x_{1}}(t) v^{-}(0)+\dot{x_{2}}(t) v^{+}(0) \\
& =\lambda x_{1}(t) v^{-}(0)-\lambda x_{2}(t) v^{+}(0)
\end{aligned}
$$

It follows that

$$
\begin{aligned}
d \lambda_{0}(v(t), A(t) v(t)) & =C\left(-\lambda v_{1}(t) v_{2}(t)-\lambda v_{1}(t) v_{2}(t)\right) \\
& =C\left(-2 \lambda r^{2}(t) \cos \theta(t) \sin \theta(t)\right) \\
& =C\left(-2 \lambda \rho^{2}(t) \cos \eta(t) \sin \eta(t)\right) \\
& =C \rho^{2}(t) \dot{\eta}(t)
\end{aligned}
$$

where $x_{1}(s)=\rho(s) \cos \eta(s), x_{2}=\rho(s) \sin \eta(s)$. The last equality follows from A.2.

We conclude that

$$
d \lambda\left(v(t), \mathcal{L}_{R}(t)\right)=C r^{2}(t)(\dot{\theta}(t)-\dot{\eta}(t)) .
$$

Assume that $t \mapsto v(t) \in \xi_{x(t)}$ satisfy $\operatorname{wind}(v, \Phi)=1$ and

$$
d \lambda\left(v(t), \mathcal{L}_{R} v(t)\right)>0, \forall t \in \mathbb{R} .
$$

By A.3, we have

$$
\dot{\theta}(t)>\dot{\eta}(t), \forall t \in \mathbb{R} .
$$

Note that $\operatorname{wind}\left(v^{ \pm}(t), \Psi\right)=1$. This follows from $\mu(P)=2$ and the geometric description of the Conley-Zehnder index given in 1.2.1. This implies that

$$
\operatorname{wind}\left(v(t), P(t)^{-1} v^{ \pm}(0)\right)=\operatorname{wind}\left(v(t), v^{ \pm}(t)\right)=0 .
$$

Now we show that for all $t \in \mathbb{R}, v(t)$ lies in regions (I) or (II). In the regions (II) and (IV), we have, by A.2,$\dot{\theta}(t)>\dot{\eta}(t)>0$. So that the existence of $t$ such that $v(t) \in(\mathrm{II})$ or (IV) would force wind $\left(v(t), P(t)^{-1} v^{ \pm}(0)\right) \geq 1$. Suppose that, for some $t_{0}, v\left(t_{0}\right)$ is in the direction of $P^{-1}\left(t_{0}\right) v^{+}(0)$, then, using 
A.2, we have $\dot{\theta}(t)>\dot{\eta}\left(t_{0}\right)$. But this would force $v(t)$ to go to (II) or (IV), which is impossible. Suppose that $v\left(t_{0}\right)$ is in the direction of $P^{-1}\left(t_{0}\right) v^{-}(0)$. Then $\dot{\theta}\left(t_{0}\right)>\dot{\eta}\left(t_{0}\right)=0$. This implies that $\theta(t)$ is growing at $t_{0}$. Since wind $\left(v(t), P(t)^{-1} v^{ \pm}(0)\right)=0$, this would force the existence of $t_{1}>t_{0}$ such that $\theta\left(t_{1}\right)=\theta\left(t_{0}\right)$ and $\dot{\theta}\left(t_{1}\right)<0$, a contradiction with $\dot{\theta}\left(t_{1}\right)>\dot{\eta}\left(t_{1}\right)=0$. We conclude that $v(t)$ lies in regions (I) or (II), for every $t \in \mathbb{R}$.

By a similar argument, we conclude that if $t \mapsto v(t) \in \xi_{x(t)}$ satisfies wind $(v, \Phi)=1$ and $d \lambda\left(v(t), \mathcal{L}_{R} v(t)\right)>0, \forall t \in \mathbb{R}$, then for every $t \in \mathbb{R}$, we have $v(t) \in(\mathrm{II})$ or $v(t) \in(\mathrm{IV})$.

Lemma A.3. Let $t \mapsto \eta(t) \in \xi$ be a vector field along $x_{2}\left(T_{2} \cdot\right)$ such that $\left\{\eta(t), R\left(x_{2}\left(T_{2} t\right)\right)\right\}$ generates $d \Psi\left(T S^{2}\right)$ along $x_{2}\left(T_{2} \cdot\right)$ and let $t \mapsto \eta^{\prime}(t) \in \xi$ be a section along $x_{2}\left(T_{2} \cdot\right)$ such that $\left\{\eta^{\prime}(t), R\left(x_{2}\left(T_{2} t\right)\right)\right\}$ generates the tangent space of $T$ along $x_{2}\left(T_{2} \cdot\right)$. Then $\operatorname{wind}\left(\eta^{\prime}, \Psi\right)=\operatorname{wind}(\eta, \Psi)=1$.

Proof. Using the fact that $\psi$ restricted to each hemisphere of $S^{2}$ is transverse to the Reeb flow, we can follow the arguments of [HSa11, Section 6] to conclude that

$$
\operatorname{wind}(\eta, \psi)=1 \text {. }
$$

In the same way, if $\nu$ is a section such that $\left\{\nu(t), R\left(x_{2}(t)\right)\right\}$ generates the tangent space of the disk $D$ along $x_{2}$, where $D$ is the disk given by the definition of 3-2-1 foliation 3.4, we have

$$
\operatorname{wind}(\nu, \Psi)=1 \text {. }
$$

Since $T$ is transverse to $D$ along $x_{2}$, we obtain

$$
\operatorname{wind}\left(\eta^{\prime}, \Psi\right)=1 \text {. }
$$

Proof of Lemma 3.9. Let $t \mapsto \eta(t) \in \xi$ be a section along $x_{2}\left(T_{2} \cdot\right)$ such that $\left\{\eta(t), R\left(x_{2}\left(T_{2} t\right)\right)\right\}$ generates $d \Psi\left(T S^{2}\right)$ along $x_{2}\left(T_{2} \cdot\right)$.

Let $u:(-\epsilon, \epsilon) \times S^{1} \rightarrow \Psi\left(S^{2}\right) ;(s, t) \mapsto u(s, t)$ be a smooth function such that

$$
\left\{\begin{array}{l}
u(0, \cdot)=x_{2 T_{2}} \\
\left.\frac{\partial}{\partial s} u(s, t)\right|_{s=0}=\eta(t)
\end{array}\right.
$$

Fix an orientation on $S^{1} \times\{0\} \subset S^{2}$ in such a way that $\left.\psi\right|_{S^{1} \times\{0\}}$ preserves orientation. Consider the closed hemispheres of $S^{2}$, that we call $H_{+}$e $H_{-}$, with the orientation induced by the orientation of $S^{1} \times\{0\}$. It follows that

$$
0<T_{2}=\int_{S^{1}} x_{2 T_{2}}^{*} \lambda=\int_{H_{ \pm}} \psi^{*} d \lambda
$$


Since $\psi$ is transverse to the Reeb flow $R_{\lambda}$ in $H_{ \pm} \backslash S^{1} \times\{0\}$, then $\psi^{*} d \lambda>0$ in $H_{ \pm} \backslash S^{1} \times\{0\}$. This implies that 0 is a local maximum of the function $s \mapsto \mathcal{A}(u(s, \cdot))$.

It follows from $(1.7)$ and 11.16$)$ that

$$
\left.\frac{d^{2}}{d s^{2}}(\mathcal{A}(u(s, \cdot)))\right|_{s=0}=T_{2} \int_{S^{1}} d \lambda\left(\eta(t), \mathcal{L}_{R} \eta(t)\right) d t<0 .
$$

Since $\left.\psi\right|_{H_{ \pm}}$is a strong transverse section, we have

$$
d \lambda\left(\eta(t), \mathcal{L}_{R} \eta(t)\right) d t<0, \forall t \in S^{1} .
$$

Let $t \mapsto \eta^{\prime}(t) \in \xi$ be a section along $x_{2}\left(T_{2} \cdot\right)$ such that $\left\{\eta^{\prime}(t), R\left(x_{2}\left(T_{2} t\right)\right)\right\}$ generates the tangent space of $T$ along $x_{2}\left(T_{2} \cdot\right)$.

Let $v:(-\epsilon, \epsilon) \times S^{1} \rightarrow T,(s, t) \mapsto v(s, t)$ be a smooth function such that

$$
\left\{\begin{array}{l}
v(0, \cdot)=x_{2 T_{2}} \\
\left.\frac{\partial}{\partial s} v(s, t)\right|_{s=0}=\eta^{\prime}(t) .
\end{array}\right.
$$

Recall that $V_{1}$ and $V_{2}$ are oriented in such a way that $d \lambda \mid V_{1,2}$ is an area form, $P_{3}$ is a positive asymptotic limit and $P_{2}$ is a negative asymptotic limit. This implies that 0 is a local minimum of the function $s \mapsto \mathcal{A}(v(s, \cdot))$.

It follows from $(1.7)$ and $(1.16)$ that

$$
\left.\frac{d^{2}}{d s^{2}}(\mathcal{A}(v(s, \cdot)))\right|_{s=0}=T_{2} \int_{S^{1}} d \lambda\left(\eta^{\prime}(t), \mathcal{L}_{R} \eta^{\prime}(t)\right) d t>0 .
$$

Since $V_{1}$ and $V_{2}$ are strong transverse sections, we have

$$
d \lambda\left(\eta^{\prime}(t), \mathcal{L}_{R} \eta^{\prime}(t)\right) d t>0, \forall t \in S^{1} .
$$

Now we can apply Proposition A.2 to the sections $\eta$ and $\eta^{\prime}$ to conclude the proof. 


\section{Appendix B}

\section{Intersection theory of punctured pseudoholomorphic curves}

In this appendix we state some results of [Sie11] that were used in the thesis. Some of the theorems stated here are simpler versions of the results of [Sie11] cited, that are enough for our purposes.

Let $(M, \lambda)$ be a contact manifold, fix $J \in \mathcal{J}(\xi, d \lambda)$ and consider the almost complex structure $\tilde{J}$ in $\mathbb{R} \times M$ defined by $(2.1)$.

In [Sie11, it is defined a generalized intersection number of pseudoholomorphic curves, denoted by [.]*[·], which counts the algebraic intersection number plus an asymptotic intersection number at punctures.

Theorem B.1. [Sie11, Corollary 5.9] Let $\tilde{u}:(\Sigma \backslash \Gamma, j) \rightarrow \mathbb{R} \times M$ and $\tilde{v}:\left(\Sigma^{\prime} \backslash \Gamma^{\prime}, j^{\prime}\right) \rightarrow \mathbb{R} \times M$ finite energy $\tilde{J}$-holomorphic curves. Assume that no component of $\tilde{u}$ or $\tilde{v}$ lies in an orbit cylinder. Then the following are equivalent:

(1) $[\tilde{u}] *[\tilde{v}]=0$.

(2) All of the following hold:

- The map u does not intersect any of the positive asymptotic limits of $v$;

- The map $v$ does not intersect any of the negative asymptotic limits of $u$;

- If $P$ is a periodic orbit so that at $z \in \Gamma, \tilde{u}$ is asymptotic $P^{m_{z}}$ and at $w \in \Gamma^{\prime}, \tilde{v}$ is asymptotic to $P^{m_{w}}$ then: If $z$ and $w$ are both positive, then $d_{0}(\tilde{u}, z)=0$ and $\frac{\operatorname{wind}\left(\nu_{P m_{z}}^{n e g},[\Phi]\right)}{m_{z}} \geq \frac{\operatorname{wind}\left(\nu_{P m_{w}}^{n e g},[\Phi]\right)}{m_{w}}$. If $z$ and $w$ are both negative, then $d_{0}(\tilde{v}, w)=0$ and $\frac{-\operatorname{wind}\left(\nu_{P m_{w}}^{\text {pos }},[\Phi]\right)}{m_{w}}=$ 
$\frac{-\operatorname{wind}\left(\nu_{P m_{z}}^{p o s},[\Phi]\right.}{m_{z}}$. If $z$ is a negative puncture and $w$ is a positive puncture, then $d_{0}(\tilde{u}, z)=d_{0}(\tilde{v}, w)=0$ and $P^{m_{z}}$ and $P^{m_{w}}$ are both even orbits, or equivalently

$$
\frac{\operatorname{wind}_{\infty}^{\Phi}(\tilde{u}, z)}{m_{z}}=\frac{\operatorname{wind}_{\infty}^{\Phi}(\tilde{u}, w)}{m_{z} w}
$$

(3) All of the following hold:

- The map u does not intersect any of the asymptotic limits of $v$.

- The map $v$ does not intersect any of the asymptotic limits of $u$.

- If $P$ is a periodic orbit so that at $z \in \Gamma, \tilde{u}$ is asymptotic to $P^{m_{z}}$ and at $w \in \Gamma^{\prime}, \tilde{v}$ is asymptotic to $P^{m_{w}}$, then $d_{0}(\tilde{u}, z)=d_{0}(\tilde{v}, w)=0$. Further, if $P$ is elliptic, then either $m_{z}$ and $m_{w}$ are both positive and $\frac{\operatorname{wind}\left(\nu_{P m_{z}}^{n e g},[\Phi]\right)}{m_{z}}=\frac{\operatorname{wind}\left(\nu_{P m_{w}}^{n e g},[\Phi]\right)}{m_{w}}$, or $m_{z}$ and $m_{w}$ are both negative and $\frac{\operatorname{wind}\left(\nu_{P}^{p o s}, m_{z},[\Phi]\right)}{m_{z}}=\frac{\operatorname{wind}\left(\nu_{P}^{p o s} m_{w},[\Phi]\right)}{m_{w}}$. If $P$ is odd hyperbolic then either $m_{z}$ and $m_{w}$ are both even or $m_{z}=m_{w}$.

Theorem B.2. [Sie11, Theorem 2.4] Let $\tilde{u}:(\Sigma \backslash \Gamma, j) \rightarrow \mathbb{R} \times M$ and $\tilde{v}:\left(\Sigma^{\prime} \backslash \Gamma^{\prime}, j^{\prime}\right) \rightarrow \mathbb{R} \times M$ asymptotically cylindrical $\tilde{J}$-holomorphic curves. Assume that no component of $\tilde{u}$ or $\tilde{v}$ lies in an orbit cylinder and that the projected curves $u$ and $v$ do not have identical image on any component of their domains. Then the following are equivalent:

(1) The projected curves $u$ and $v$ do not intersect;

(2) All of the following hold:

- The map u does not intersect any of the positive asymptotic limits of $v$;

- The map $v$ does not intersect any of the negative asymptotic limits of $u$;

- If $P$ is a periodic orbit so that at $z \in \Gamma, \tilde{u}$ is asymptotic $P^{m_{z}}$ and at $w \in \Gamma^{\prime}, \tilde{v}$ is asymptotic to $P^{m_{w}}$ then: If $z$ and $w$ are both positive or both negative punctures, then $\frac{\operatorname{wind}_{\infty}^{\Phi}(\tilde{u}, z)}{m_{z}} \geq \frac{\operatorname{wind}_{\infty}^{\Phi}(\tilde{v}, w)}{m_{w}}$. If $z$ is a negative puncture and $w$ is a positive puncture, then

$$
\frac{\operatorname{wind}_{\infty}^{\Phi}(\tilde{u}, z)}{m_{z}}=\frac{\left\lfloor\mu^{\Phi}\left(P^{m_{z}}\right) / 2\right\rfloor}{m_{z}}=\frac{\left\lfloor\mu^{\Phi}\left(P^{m_{w}}\right)\right\rfloor}{m_{w}}=\frac{\operatorname{wind}_{\infty}^{\Phi}(\tilde{v}, w)}{m_{w}}
$$

(3) All of the following hold: 
- The map $u$ does not intersect any of the asymptotic limits of $v$.

- The map $v$ does not intersect any of the asymptotic limits of $u$.

- If $P$ is a periodic orbit so that at $z \in \Gamma, \tilde{u}$ is asymptotic to $P^{m_{z}}$ and at $w \in \Gamma^{\prime}, \tilde{v}$ is asymptotic to $P^{m_{w}}$, then $\frac{\operatorname{wind}_{\infty}^{\Phi}(\tilde{u}, z)}{m_{z}}=\frac{\operatorname{wind}_{\infty}^{\Phi}(\tilde{v}, w)}{m_{w}}$.

Definition B.3 ([Sie11]). Let $\tilde{u}: \Sigma \backslash \Gamma^{\prime} \rightarrow \mathbb{R} \times M$ and $\tilde{v}: \Sigma^{\prime} \backslash \Gamma^{\prime} \rightarrow \mathbb{R} \times M$ be finite energy $\tilde{J}$-holomorphic curves asymptotic to the same nondegenerate periodic orbit $P \in \mathcal{P}(\lambda)$ at certain punctures $z \in \Gamma$ and $w \in \Gamma^{\prime}$. We say that $\tilde{u}$ and $\tilde{v}$ approach $P$ in the same direction at these punctures if $\eta_{u}=c \eta_{v}$ for $c>0$, where $\eta_{u}$ and $\eta_{v}$ are the asymptotic eigensections of $\tilde{u}$ at $z$ and of $\tilde{v}$ at $w$ respectively, defined in Theorem 2.11. In case $\eta_{u}=c \eta_{v}$ with $c<0$, we say that $\tilde{u}$ and $\tilde{v}$ approach $P$ in opposite directions.

Theorem B.4. [Sie11, Theorem 2.5] Let $P$ be a simple even orbit and let $\tilde{u}: \Sigma \backslash \Gamma \rightarrow \mathbb{R} \times M$ and $\tilde{v}: \Sigma \backslash \Gamma^{\prime} \rightarrow \mathbb{R} \times M$ be connected finite energy $\tilde{J}$-holomorphic curves. Assume that at punctures $z \in \Gamma$ and $w \in \Gamma^{\prime}, \tilde{u}$ and $\tilde{v}$ approach $P$ in the same direction, and that there do not exist neighborhoods $U$ of $z$ and $V$ of $w$ so that $u(U \backslash\{z\})=v(V \backslash\{w\})$. Then

$$
[\tilde{u}] *[\tilde{v}]>0 \text {. }
$$

Theorem B.5. SSie11, Theorem 2.6] Let $\tilde{u}: \Sigma \backslash \Gamma \rightarrow \mathbb{R} \times M$ be a connected simple finite energy $\tilde{J}$-holomorphic curve. Assume that $\tilde{u}$ does not have image contained in an orbit cylinder. Then the following are equivalent:

(1) The projected map $u: S \backslash \Gamma \rightarrow M$ is an embedding.

(2) The algebraic intersection number $\operatorname{int}\left(\tilde{u}, \tilde{u}_{c}\right)$ between $\tilde{u}$ and an $\mathbb{R}$-translate $\tilde{u}_{c}=(a+c, u)$ is zero for all $c \in \mathbb{R} \backslash\{0\}$.

(3) All of the following hold:

- $u$ does not intersect any of its asymptotic limits.

- If $P$ is a periodic orbit so that $u$ is asymptotic at $z \in \Gamma$ to $P^{m_{z}}$ and $u$ is asymptotic at $w \in \Gamma$ to $P^{m_{w}}$, then $\frac{\operatorname{wind}_{\infty}(\tilde{u}, z)}{m_{z}}=\frac{\operatorname{wind}_{\infty}(\tilde{u}, w)}{m_{w}}$.

If (1), (2) or (3) holds, then

- The map $\tilde{u}$ is an embedding.

- The projected map $u$ is an immersion which is everywhere transverse to the Reeb vector field.

- For each $z \in \Gamma$, we have $\operatorname{gcd}\left(m_{z}, \operatorname{wind}_{\infty}(\tilde{u}, z)\right)=1$. 


\section{Bibliography}

[AD14] M. Audin and M. Damian. Morse theory and Floer homology. Springer, 2014.

$\left[\mathrm{BEH}^{+} 03\right]$ F. Bourgeois, Y. Eliashberg, H. Hofer, K. Wysocki, and E. Zehnder. Compactness results in symplectic field theory. Geometry and Topology, 7(2):799-888, 2003.

[Ben83] D. Bennequin. Entrelacements et equations de pfaff. Astérisque, 107-108:87-161, 1983.

[dPSa18] N. de Paulo and P. A. S. Salomão. Systems of transversal sections near critical energy levels of hamiltonian systems in $\mathbb{R}^{4}$. Memoirs of the American Mathematical Society, 252(1202), 2018.

[Dra04] D. Dragnev. Fredholm theory and transversality for noncompact pseudoholomorphic maps in symplectizations. Communications on pure and applied mathematics, 57(6):726-763, 2004.

[Eli92] Y. Eliashberg. Contact 3-manifolds twenty years since j. martinet's work. Ann. Inst. Fourier (Grenoble), 42(1-2):165-192, 1992.

[Etn06] J.B. Etnyre. Contact manifolds. In Jean-Pierre Françoise, Gregory L. Naber, and Tsou Sheung Tsun, editors, Encyclopedia of Mathematical Physics, pages 631 - 636. Academic Press, Oxford, 2006.

[Fra92] J. Franks. Geodesics on $S^{2}$ and periodic points of annulus homeomorphisms. Inventiones mathematicae, 108(1):403-418, 1992.

[FS18] J. Fish and R. Siefring. Connected sums and finite energy foliations i: Contact connected sums. Journal of Symplectic Geometry, 16(6):1639-1748, 2018. 
[Gei08] H. Geiges. An introduction to contact topology, volume 109. Cambridge University Press, 2008.

[GP10] V. Guillemin and A. Pollack. Differential topology, volume 370. American Mathematical Soc., 2010.

[Gro85] M. Gromov. Pseudo holomorphic curves in symplectic manifolds. Inventiones Mathematicae, 82:307-347, 1985.

[HK99] H. Hofer and M. Kriener. Holomorphic curves in contact dynamics. In Proceedings of Symposia in Pure Mathematics, volume 65, pages 77-132. American Mathematical Society, 1999.

[HLSa15] U. Hryniewicz, J. Licata, and P. A. S. Salomão. A dynamical characterization of universally tight lens spaces. Proceedings of the London Mathematical Society, 110(1):213-269, 2015.

[Hof93] H. Hofer. Pseudoholomorphic curves in symplectizations with applications to the weinstein conjecture in dimension three. Inventiones mathematicae, 114(1):515-563, 1993.

[Hry12] U. Hryniewicz. Fast finite-energy planes in symplectizations and applications. Transactions of the American Mathematical Society, 364(4):1859-1931, 2012.

[Hry14] U. Hryniewicz. Systems of global surfaces of section for dynamically convex reeb flows on the 3-sphere. Journal of Symplectic Geometry, 12(4):791-862, 2014.

[HS16] U. Hryniewicz and P. A. S. Salomão. Elliptic bindings for dynamically convex reeb flows on the real projective three-space. Calculus of Variations and Partial Differential Equations, 55(2):43, Apr 2016.

[HSa09] U. Hryniewicz and P. A. S Salomão. Uma introdução à geometria de contato e aplicações à dinâmica Hamiltoniana. Impa, 2009.

[HSa11] U. Hryniewicz and P. A. S. Salomão. On the existence of disk-like global sections for reeb flows on the tight 3 -sphere. Duke Math. J., 160(3):415-465, 2011.

[HSa18] U. Hryniewicz and P. Salomão. Global surfaces of section for reeb flows on dimension three and beyond. In Proceedings of the International Congress of Mathematicians 2018, volume 2, pages 959-986, 2018. 
[HWZ95a] H. Hofer, K. Wysocki, and E. Zehnder. A characterization of the tight 3-sphere. Duke Math. J., 81(1):159-226, 1995.

[HWZ95b] H. Hofer, K. Wysocki, and E. Zehnder. Properties of pseudoholomorphic curves in symplectisations II: Embedding controls and algebriac invariants. Geometric and Functional Analysis, $5(2): 270-328,1995$.

[HWZ96] H. Hofer, K. Wysocki, and E. Zehnder. Properties of pseudoholomorphic curves in symplectisations I: Asymptotics. Annales de l'Institut Henri Poincaré C, Analyse Non Linéaire, 13(3):337379, 1996.

[HWZ98] H. Hofer, K. Wysocki, and E. Zehnder. The dynamics on threedimensional strictly convex energy surfaces. Annals of Mathematics, 148(1):197-289, 1998.

[HWZ99a] H. Hofer, K. Wysocki, and E. Zehnder. A characterization of the tight 3-sphere II. Communications on pure and applied mathematics, 52(9):1139-1177, 1999.

[HWZ99b] H. Hofer, K. Wysocki, and E. Zehnder. Properties of pseudoholomorphic curves in symplectizations III: Fredholm theory. Topics in nonlinear analysis, 35:381-475, 1999.

[HWZ03] H. Hofer, K. Wysocki, and E. Zehnder. Finite energy foliations of tight three-spheres and hamiltonian dynamics. Annals of Mathematics, 157(1):125-255, 2003.

[McD91] D. McDuff. The local behaviour of holomorphic curves in almost complex 4-manifolds. J. Differential Geom., 34(1):143-164, 1991.

[MS04] D. McDuff and D. Salamon. J-holomorphic curves and symplectic topology, volume 52 of Colloquium publications. American Mathematical Society, 2004.

[MS17] D. McDuff and D. Salamon. Introduction to symplectic topology. Oxford University Press, 2017.

[Nel13] J. Nelson. Applications of automatic transversality in contact homology. PhD thesis, University of Wisconsin-Madison, 2013.

[Nel15] J. Nelson. Automatic transversality in contact homology I: regularity. In Abhandlungen aus dem Mathematischen Seminar der Universität Hamburg, volume 85, pages 125-179. Springer, 2015. 
[Rol03] D. Rolfsen. Knots and links. American Mathematical Soc., 2003.

[Sie08] R. Siefring. Relative asymptotic behavior of pseudoholomorphic half-cylinders. Communications on Pure and Applied Mathematics: A Journal Issued by the Courant Institute of Mathematical Sciences, 61(12):1631-1684, 2008.

[Sie11] R. Siefring. Intersection theory of punctured pseudoholomorphic curves. Geometry \&5 Topology, 15(4):2351-2457, 2011.

[Tay11] M. E. Taylor. Partial Differential Equations I: Basic Theory (Applied Mathematical Sciences). Springer New York, 2011.

[Wen05] C. Wendl. Finite Energy Foliations and Surgery on Transverse Links. PhD thesis, New York University, 2005.

[Wen16] C. Wendl. Lectures on symplectic field theory. arXiv preprint arXiv:1612.01009, 2016. 